\title{
On The Chloride DePendence OF Vesicular Glutamate Transport
}

\author{
Dissertation \\ zur Erlangung des Doktorgrades \\ der Mathematisch-Naturwissenschaftlichen Fakultäten \\ der Georg-August-Universität zu Göttingen
}

vorgelegt von

Stephan Schenck

aus Hamburg

Göttingen 2009 
D7

Referent: Prof. Dr. Reinhard Jahn

Koreferent: Prof. Dr. Ralf Ficner

Tag der mündlichen Prüfung: 26.06.2009 
This work was conducted at the

\title{
TOKYO MEDICAL AND DENTAL UNIVERSITY
}

\author{
1-5-45 Yushima, Bunkyo-ku, Tokyo \\ in the laboratory of
}

Dr. Shigeo Takamori 


\section{Abbreviations}

\begin{tabular}{|c|c|}
\hline ADP & Adenosin-diphosphat \\
\hline ATP & Adenosin-triphosphat \\
\hline $\mathrm{AU}$ & arbitary units \\
\hline$\beta-\mathrm{CD}$ & 2,6-di-O-methyl- $\beta$-cyclodextrin \\
\hline $\mathrm{CLC} / \mathrm{ClC}$ & Intracellular chloride channel \\
\hline DDM & n-dodecyl- $\beta$-D-maltopyranoside \\
\hline DTT & dithiothreitol \\
\hline$\Delta \mathrm{pH}$ & pH gradient \\
\hline$\Delta \Psi$ & membrane potential \\
\hline$\Delta \mu_{\mathrm{H}+}$ & proton electrochemical gradient \\
\hline EDTA & ethylenediaminetetraacetate \\
\hline EB & Evan's Blue \\
\hline FCCP & $p$-(trifluoromethoxy)phenylhydrazone \\
\hline $\mathrm{F}_{0} \mathrm{~F}_{1}$ & F-ATPase (ATP-synthase) \\
\hline Fig. & Figure \\
\hline GABA & $\gamma$-aminobutyric acid \\
\hline Glu & glutamate \\
\hline Gluc & gluconate \\
\hline HEPES & 4-(2-hydroxyethyl)-1-piperazineethanesulfonic acid \\
\hline HRP & horseradish peroxidase \\
\hline $\mathrm{kDa}$ & kilodalton \\
\hline Kgluc & potassium gluconate \\
\hline $\mathrm{KO}$ & knock out \\
\hline LP2 & light membrane pellet 2 \\
\hline MOPS & 3-(N-morpholino)propanesulfonic acid \\
\hline NIG & nigericin \\
\hline OG & $\mathrm{n}$ - $\beta$-octylglucoside \\
\hline PAGE & polyacrylamide gel electrophoresis \\
\hline PMSF & phenylmethylsulfonylfluoride \\
\hline SDS & sodium dodecyl sulfate \\
\hline SEC & size exclusion chromatography \\
\hline SLC /Slc & solute carrier family \\
\hline SV & synaptic vesicle \\
\hline TEV & tobacco etch virus \\
\hline Tris & tris(hydroxymethyl)aminomethane \\
\hline $\mathrm{TF}_{0} \mathrm{~F}_{1}$ & thermophilic F-ATPase (ATP-synthase) \\
\hline $\mathrm{U}$ & unit of enzyme activity \\
\hline VAchT & vesicular actylcholine transporter \\
\hline VAL & valinomycin \\
\hline V-ATPase & vacuolar ATPase \\
\hline VGAT & vesicular GABA transporter \\
\hline VGLUT & vesicular glutamate transporter \\
\hline VIAAT & vesicular inhibitory amino acid transporter \\
\hline VMAT & vesicular monoamine transporter \\
\hline WT & wild type \\
\hline $\mathrm{w} / \mathrm{v}$ & weight per volume \\
\hline
\end{tabular}




\title{
TABLE OF CONTENTS
}

\begin{abstract}
Abbreviations
Table of contents

Table of Figures $\quad$ viii-ix
\end{abstract}

1. Introduction

1.1. The synaptic vesicle cycle: Exocytosis, endocytosis, and transmitter loading of synaptic vesicles are the basis of neurotransmission $\ldots \ldots \ldots \ldots \ldots \ldots \ldots$

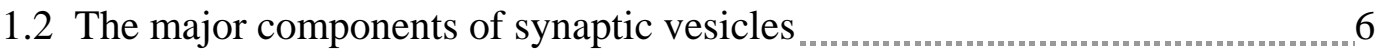

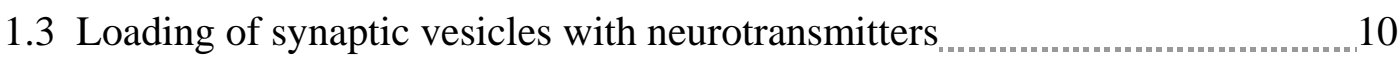

1.3.1 Driving the uptake: Generation of an proton-electrochemical

Gradient

1.3.2 Glutamate is the major excitatory transmitter in the vertebrate brain and translocated by vesicular glutamate transporters (VGLUTs)

1.3.3 The VGLUT isoforms and their distribution in the mammalian brain

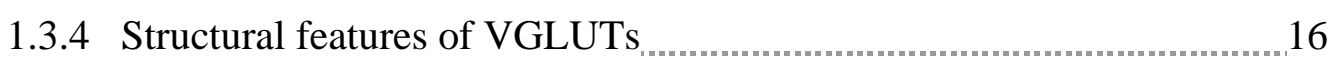

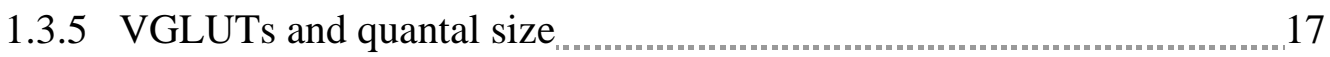

1.3.6 The chloride dependence of VGLUTs: Implications for the energy requirements of glutamate translocation

1.3.7 The energy requirements of other major vesicular neurotransmitter transporters

1.4 Ion permeabilities of intracellular compartments and their influence on the electrochemical gradient 24

1.4.1 ClC-3, a candidate for the chloride channel on synaptic vesicles 27

1.5 Synaptic vesicle acidifcation and glutamate uptake: Are glutamate loading and anion shunting separate units ? 30

1.6 Aim of this work 31 


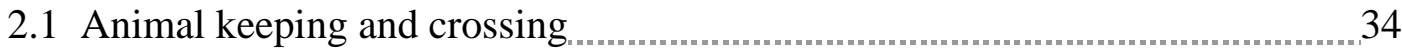

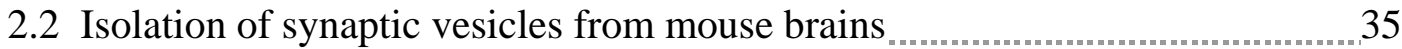

2.3 Heterologous expression and purification of a proton pump

2.3.1 Purification of E. coli wild type ATP-Synthase $\left(\mathrm{F}_{0} \mathrm{~F}_{1}\right)$. 36

2.3.2 Purification of Bacillus sp. PS3 thermophilc ATP-Synthase $\left(\mathrm{TF}_{0} \mathrm{~F}_{1}\right)$ 37

2.4 Heterologous expression and purification of VGLUTs in tsA201 cells 39

2.4.1 Cell culture and transfection 39

2.4.2 Purification of recombinant tagged VGLUTs 40

2.5 Reconstitution of a proton pump and VGLUTs into artificial membranes 41

2.6 Measurement of vesicle acidification with acridine orange 42

2.6.1 KCl- and KGlu-dependent acidification of LP2-fractions. 42

2.6.2 KCl-dependent acidification of reconstituted vesicles, 43

2.7 Measurement of glutamate uptake 43

2.7.1 Control of the intra- and extravesicular ion composition for glutamate uptake experiments 44

2.8 Electron microscopy 45

2.9 Entrapment of $\left[{ }^{14} \mathrm{C}\right]$-glucose in liposomes for the determination of the intravesicular volume 45

2.10 Miscellaneous general methods

2.10.1 Protein measurement, electrophoresis and imunoblotting .......... 46

2.10.2 Antibodies

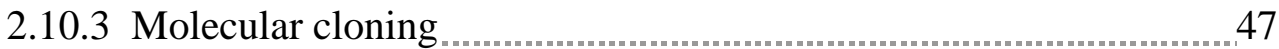

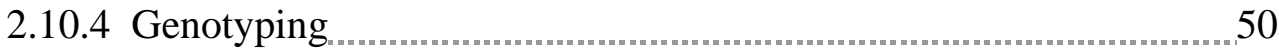


3.1 Analysis of crude synaptic vesicles isolated from $\mathrm{Clcn}^{-{ }^{--}}$- and $\mathrm{Vglut}^{-1-}$ -brains

3.1.1 Measurement of the $\mathrm{Cl}^{-}$- and glutamate-dependent acidification of

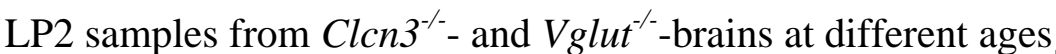
54

3.1.2 Relevant protein levels in LP2-samples of $\mathrm{Clcn}^{-/-}$- and $\mathrm{Vglut}^{-{ }^{-}}$ -brains 58

3.2 Anion dependence of glutamate induced acidifcation in LP2 samples 59

3.3 Estimation of the copy number of $\mathrm{ClC}-3$ on synaptic vesicles 61

3.4 Purification of proton pumps, VGLUT1 and liposome formation 63

3.4.1 Purification of proton pumps 63

3.4.1.1 Purification of $\mathrm{F}_{0} \mathrm{~F}_{1}$ wild type ATP-synthase of E. coli 64

3.4.1.2 Purification of recombinant His-tagged ATP-synthase from the thermophilic Bacillus sp. PS3 $\left(\mathrm{TF}_{0} \mathrm{~F}_{1}\right)$

3.4.2 Heterologous expression and purification of VGLUT1 in tsA201 cells

3.4.3 Formation of liposomes and comparison of the $\mathrm{F}_{0} \mathrm{~F}_{1}$ and $\mathrm{TF}_{0} \mathrm{~F}_{1}$ ATP-synthases 71

3.5 Co-reconstitution of VGLUT1 and TF0F1 and acidification of liposomes $\ldots . . . .75$

3.5.1 VGLUT1 exhibits a $\mathrm{Cl}^{-}$conductance in reconstituted liposomes.. .76

3.5.2 The $\mathrm{Cl}^{-}$permeabilty of reconstituted liposomes is

VGLUT1-dose-dependendent

3.5.3 Removal of VGLUT1 of the liposome premix strongly reduces the $\mathrm{Cl}^{-}$conductance 78

3.5.4 The $\mathrm{Cl}^{-}$conductivity co-migrates with VGLUT1 in size exclusion chromatography

3.5.5 The $\mathrm{Cl}^{-}$conductance in VGLUT1 is largely independent of the intravesicular anion 
3.6 Glutamate uptake of VGLUT1 - $\mathrm{TF}_{0} \mathrm{~F}_{1}$ liposomes

3.6.1 The intravesicular ion composition is an unknown factor for glutamate transport

3.6.2 Glutamate uptake into liposomes depends on ATP and is enhanced by intravesicular $\mathrm{Cl}^{-}$

3.6.3 The uptake kinetics show an increase of $v_{\max }$ for $\mathrm{Cl}^{-}$loaded liposomes compared to gluconate loaded liposomes 85

3.6.4 Influence of extravesicular $\mathrm{Cl}^{-}$on the glutamate uptake 86

3.6.4.1 The biphasic dependence on extravesicular $\mathrm{Cl}^{-}$is preserved in the reconstituted system 86

3.6.4.2 $\mathrm{Cl}^{-}$loaded liposomes show higher uptake at all extravesicular $\mathrm{Cl}^{-}$concentrations 87

3.6.5 $\Delta \psi$-driven uptake is strongly facilitated by intravesicular $\mathrm{Cl}^{-}$ 88

- 3.6.5.1 Glutamate uptake depends on the actual internal $\mathrm{Cl}^{-}$ concentration: Indications for a glutamate $/ \mathrm{Cl}^{-}$antiport activity in VGLUT1

3.7 Comparison of the internal volumes of $\mathrm{Cl}^{-}$- and -gluconate loaded liposomes 90

3.7.1 Electron micrographs reveal only minor differences in the volume of Cl- and gluconate-loaded liposomes

3.7.2 Direct measurement of the total liposome volume with a radiotracer

3.8 Heterologous expression and purification of VGLUT isoforms and homologues

3.8.1 Expression of VGLUT from Drosophila melanogaster (DVGLUT)

3.8.2 Expression of eat-4: The Caenorhabditis elegans homologue of VGLUTs.

3.8.3 Expression of murine VGLUT2 95 
3.9 Characterization of glutamate transport by DVGLUT, eat-4 and VGLUT2 _...95

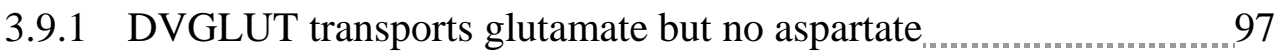

3.9.2 DVGLUT shows a biphasic dependence on extravesicular $\mathrm{Cl}^{-}$ and only minimal dependence on the intravesicular anion $\ldots . . . . . . . .98$

3.9.3 The glutamate translocation by DVGLUT and eat-4 depends largely on $\Delta \mathrm{pH}$

3.9.4 Murine VGLUT2 shows the same energy dependence pattern as VGLUT1 and exhibits enhanced transport by intravesicluar $\mathrm{Cl}^{-}$

3.9.5 DVGLUT exhibits a $\mathrm{Cl}^{-}$shunting activity in liposome acidification 101

3.10 The differences of invertebrate and vertebrate VGLUTs are useful for structure-function studies 102

3.10.1 Topology prediction of VGLUT1 using the TMHMM server 103

3.10.2 Alignment of vertebrate and evertebrate VGLUTs reveals only few non-conserved residues in the transmembrane domains 103

3.10.3 Heterologous expression of selected VGLUT1 mutant proteins 106

3.10.4 Characterization of VGLUT1-mutant proteins by their energy dependence for glutamate translocation 107

4. Discussion 109

4.1 The comparison of Vglut $^{-/-}$and $\mathrm{Clcn}^{-/}$strains clearly favors a role for VGLUT1 in $\mathrm{Cl}^{-}$shunting on SVs. 109

4.2 Reconstitution of VGLUT1 into artificial liposomes confirms a $\mathrm{Cl}^{-}$conductance in vitro 112

4.3 The glutamate loading mechanism of SVs: The role of extra- and intravesicular $\mathrm{Cl}^{-}$ions. 113 
4.4 $\mathrm{A} \mathrm{Cl}^{-} /$glutamate antiport could underlie the enhancement of glutamate transport in $\mathrm{Cl}^{-}$loaded liposomes

4.5 Implications of the $\mathrm{Cl}^{-} /$glutamate antiport activity for the loading of glutamate in the synapse

4.6 The lack of enhanced transport by intravesicular $\mathrm{Cl}^{-}$in invertebrate VGLUTs suggests different loading kinetics and smaller quantal size

4.7 $\mathrm{Are}^{-}$shunting and $\mathrm{Cl}^{-} /$glutamate antiport based on different mechanisms in VGLUT1?

5. Summary

6. Zusammenfassung

7. 要約

8. References

List of Publications 150

Acknowledgements 


\section{Table of figures}

Fig. Title $\underline{\text { Page }}$

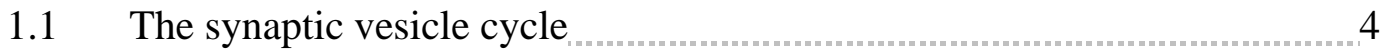

1.2 A molecular model of an average synaptic vesicle $\ldots$

1.3 A charged synaptic vesicle cut into half 8

1.4 The biphasic dependence on extravesicular $\mathrm{Cl}^{-}$of glutamate uptake $\ldots . . . .20$

1.5 Current working models for the loading of small classical transmitters ..... 23

1.6 Acidification of intracellular organelles

1.7 Determinants of vesicle acidification 26

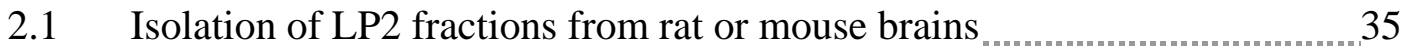

2.2 Cassette for VGLUT expression in the cloning vector pBluescript SK+ +48

2.3 Sequence details for the cloning of SBP-tagged VGLUT genes ............49

2.4 Genotyping of Vglut1 and Clcn3 mouse strains

3.1 Acidification of SVs (LP2) from $\mathrm{Clcn}^{-/}$brains in the presence of external $\mathrm{Cl}^{-}$or glutamate

3.2 Acidification of SVs (LP2) from Vglut $^{-1-}$ brains in the presence of external $\mathrm{Cl}^{-}$or glutamate

3.3 Quantitative summary of $\mathrm{Cl}$ - and glutamate-induced acidification of LP2 samples from VGLUT1- and ClC-3-deficient mice

3.4 Quantitative comparison of protein levels for relevant SV-proteins in SV fractions derived from brains of Vglut $^{-/-}$and $\mathrm{Clcn}^{-/-}$mice $\ldots . . . . .57$

3.5 calibration curves for the antibodies and antigens used to characterize

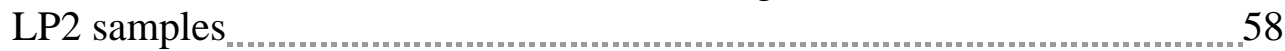

3.6 Anion dependence of glutamate induced acidification in LP2 samples .....60

3.7 Enrichment blot of SVs probed with anti-ClC-3 antibody $\ldots \ldots \ldots \ldots \ldots \ldots \ldots \ldots$

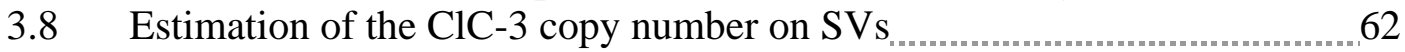

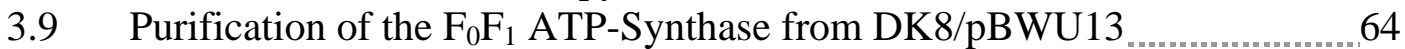

3.10 Anion exchange chromatography of $\mathrm{TF}_{0} \mathrm{~F}_{1} \quad 65$

3.11 Size exclusion chromatography of $\mathrm{TF}_{0} \mathrm{~F}_{1} \ldots 66$

3.12 Expression of VGLUT1 in tsA201 cells using transient transfection ....... 67

3.13 Purification of VGLUT1 transiently expressed in tSA201 cells

3.14 Purification of the VGLUT1 mutant proteins R176E and H120A

3.15 Electrogenic properties of $\mathrm{F}_{0} \mathrm{~F}_{1}$ and $\mathrm{TF}_{0} \mathrm{~F}_{1}$ - ATP synthase preparations $\ldots 1$

3.16 Influence of DDM, cholesterol and $\beta-\mathrm{CD}$ on proton coupling on

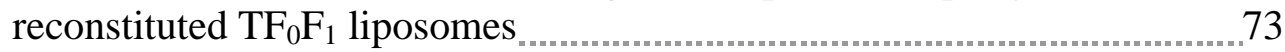

3.17 Flotation density gradient of reconstituted VGLUT1 liposomes $\ldots \ldots \ldots \ldots \ldots . . .75$

3.18 Purified VGLUT1 exhibits a chloride conductance $\ldots . .76$

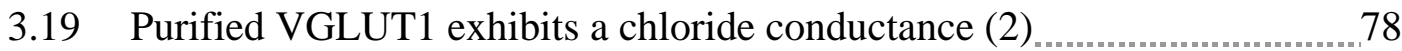




\section{Table of figures (2)}

Fig. Title

3.20 Specific removal of VGLUT1 from the liposome premix reduces the $\mathrm{Cl}^{-}$conductance

3.21 Size exclusion chromatography of purified VGLUT1

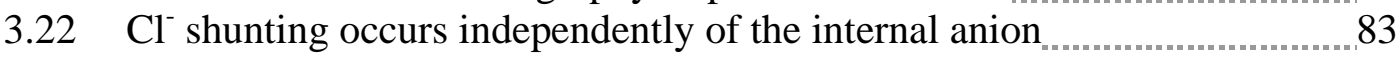

3.23 Enhancement of glutamate transport into liposomes by high luminal $\mathrm{Cl}^{-}$concentrations

3.24 Kinetics of glutamate uptake in the presence of luminal gluconate or $\mathrm{Cl}^{-} 86$

3.25 Dependence of glutamate transport on extravesicular $\mathrm{Cl}^{-}$and contribution of $\Delta \mathrm{pH}$ and $\Delta \Psi$ on the total uptake 87

3.26 $\Delta \Psi$ is indispensable for glutamate uptake in the absence of external $\mathrm{Cl}^{-} \quad 88$

3.27 Intravesicular $\mathrm{Cl}^{-}$could be a substrate in an antiport mode of VGLUT1 89

3.28 Binding of glutamate and $\mathrm{Cl}^{-}$to VGLUT1 is differently affected by Evan's Blue

3.29 Relative volumes of liposomes formed in KGluc or $\mathrm{KCl}$ containing buffers

3.30 Relative volumes of liposomes formed in KGluc or $\mathrm{KCl}$ containing buffers (2)

3.31 Expression and purification of DVGLUT in tsA201 cells

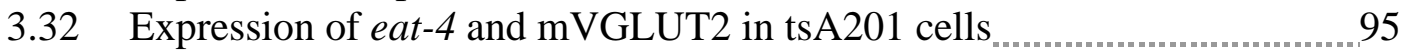

3.33 Purified isoforms of VGLUT1

3.34 Specificity of glutamate uptake by DVGLUT

3.35 Glutamate uptake of reconstituted DVGLUT

3.36 Comparison of the $\mathrm{Glu} / \mathrm{Cl}$ - antiport activity and dependence on $\Delta \Psi$ and $\Delta \mathrm{pH}$ for four different vesicular glutamate transporters $\ldots \ldots \ldots \ldots \ldots \ldots \ldots$

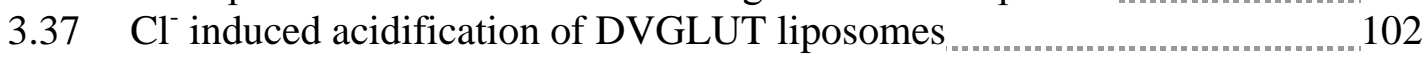

3.38 Topology prediction of VGLUT1 using TMHMM

3.39 Sequence alignment of eat-4, DVGLUT and rVGLUT1 + $2 \ldots \ldots \ldots \ldots \ldots$

3.40 Expression and purification of 5 mutant versions of VGLUT1 $\ldots . . . . . . . . . . . . .106$

3.41 Characterisation of VGLUT1-mutant proteins by their energy dependence for glutamate translocation 108

4.1 Vesicle pools of whole brain SV isolates in the VGLUT1 KO can explain the decreased acidification.

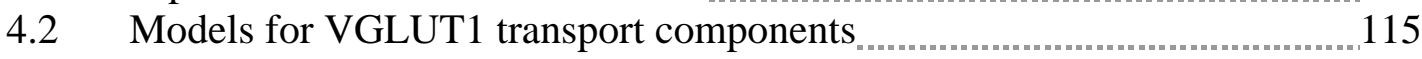

4.3 Illustration of glutamate loading at the nerve terminal during recycling of SVs

4.4 Refined model of the loading mechanism by DVGLUT and VGLUT1/2 119 


\section{INTRODUCTION}

\subsection{The synaptic vesicle cycle: Exocytosis, endocytosis, and transmitter loading of synaptic vesicles are the basis of neurotransmission}

The eukaryotic cell stands out due its extensive intracellular membrane system that builds a number of organelles and is the basis of various cellular processes that do not exist in prokaryotes. The intracellular membrane system can be divided into morphologically distinct compartments, e.g. the nuclear envelope, the endoplasmatic reticulum, the Golgi apparatus, vacuoles and secretory granules that give rise to the enourmous repertoire of biochemical activities in eukaryotes. Yet, these compartments are all interconnected due to the dynamic nature of the membraneous compartments that constantly exchange matter by trafficking events. For instance, the components of one compartment, e.g. certain lipids and proteins, are recognized by a specialized machinery of trafficking proteins that accomplish the transfer to a defined target. The crowded environment of the cell immediately implies that these processes must be tightly regulated to achieve the desired specificity for cargo transport. Although not fully separated, the trafficking of membraneous compartments or organelles is classically divided into the secretory pathway that ends with the secretion into the extracellular space and the endocytic pathway, that starts at the plasma membrane and often ends in lysosomal compartments to degrade proteins and membranes.

Two major cellular functions form the basis of these trafficking events in order to transfer membraneous patches from a donor compartment to a target zone: Fusion and fission of membranes. In endocytic events as well as intracellular budding processes, a machinery of proteins recognizes membrane patches that bear distinct signatures such as certain lipids and amino acid sequences to perform the budding procedure. These proteins must ensure 
the recruitment of specific cargo, curving of membranes to overcome energetic barriers during the formation of a membrane bud, and finally cleave off the vesicle membrane area by fission. In fusion events a donor vesicle will be directed to a target site. Lipids and protein receptors are involved in the correct trafficking and then make such a vesicle fusion competent by docking and priming. Finally the energy barrier to fuse two lipid membranes has to be overcome, which is again greatly facilitated by special proteins. Characteristic, not only to the fusion event, but also to the fission, is the non-leaky nature of these processes. The luminal contents never mix with the cytosol.

The transport of solutes across the lumen is mediated by various ion pumps, channels and transporters that specifically catalyze the transfer of ions, organic molecules and even protein chains.

The chemical transmission of signals between neurons, or neurons and other target cells is essentially built on the above mentioned basic features of the secretory and endocytic pathways. In the billions of neurons that comprise the human brain secretory and endocytic events at the contact points of neurons, the synapses, have reached a remarkable degree of specialization and speed that gives rise to the sensory and cognitive abilities that are characteristic for the CNS. Albeit resembling secretory processes in general, the chemical transmission at the synapse is unique due to its enourmous speed and synchronization which makes it possible to encode a message from one neuron to another in high temporal resolution. Since the neurotransmission is based on the repetitive fusion of a high number of vesicles with the plasma membrane, it is mandatory that the vesicles are regenerated by recycling at the restricted and isolated area of the synaptic terminal in order to refill the pool of transmitter-storing vesicles. The synaptic transmission is therefore a highly regulated interplay between exocytic and endocytic events in a small area of the cell that is specialized on speed and synchrony. This cycle of exocytosis and endocytosis at the synapse that underlies the quantal transmission of signals has therefore been termed synaptic vesicle cycle (Sudhof, 2004).

Since the work of Katz and colleagues (Del Castillo and Katz, 1956), it is known that the neurotransmission is encoded in discrete packages of neurotransmitters, the quanta. These packages are the contents of synaptic vesicles (SVs), organelles that store the neurotransmitter which is released into the the synaptic cleft to diffuse to receptors on the postsynaptic membrane. The quantal release and the identification of SVs as the storage 
compartment for transmitters gave rise to the nowadays widely accepted theory of chemical neurotransmission. A large body of evidence supports this theory. For example, it has been shown that isolated SVs contain high levels of neurotransmitters, far above the cytosolic concentration of these substances (Burger et al., 1989; Whittaker and Sheridan, 1965). Electron microscopy of synapses revealed the high number of SVs in terminals, their close opposition to the membrane and even fusion intermediates (the omegastructure) (Heuser and Reese, 1973; Heuser et al., 1979). These experiments also showed that a soluble label (horseradish peroxidase, HRP) can be taken up by recycling synaptic vesicles, providing evidence that the quantal release is based on exocytosis-endocytosis cycles of membraneous compartments. Furthermore, it has been shown that bacterial neurotoxins cleave proteins that are necessary for the fusion of synaptic vesicles with the plasma membrane, the Soluble $N$-ethylmaleimide sensitive factor (NSF) Attachment protein REceptors (SNAREs) (Schiavo et al., 2000). The activity of these toxins abolishes neurotransmission by the blockade of fusion, which is only compatible with storage of neurotransmitter quanta in SVs. The understanding of neuronal transmission has been greatly facilitated by the possibilty to isolate SVs with high purity from brains by subcellular fractionation (Huttner et al., 1983; Nagy et al., 1976). This enabled the identification of key proteins that are located on synaptic vesicles (Baumert et al., 1989; Brose et al., 1990; Sudhof et al., 1987) and necessary for its function, but also to investigate the loading mechanism and transmitter specificity. The quantal nature of transmission is also compatible with the uniform size of SVs of $\sim 40 \mathrm{~nm}$ (Takamori et al., 2006).

The synaptic vesicle cycle describes the key events of neurotransmission and can be divided into three major events (Fig. 1.1). It starts with the fusion of a transmitter filled vesicle upon arrival of a trigger signal, which is an action potential that propagated along the axon to reach the synaptic terminal. The voltage change across the membrane then opens $\mathrm{Ca}^{2+}$ channels, that trigger the fusion within milliseconds by the entry of $\mathrm{Ca}^{2+}$ ions into the presynaptic terminal. The $\mathrm{Ca}^{2+}$ ions are binding to calcium sensor proteins (the synaptotagmins) located on the surface of SVs which then elicit the synchronous fusion of competent SVs with the plasma membrane in an intricate and still debated manner (Martens and McMahon, 2008; Rizo and Rosenmund, 2008; Sudhof and Rothman, 2009). The fusion event is depending on the SNARE proteins, membrane-anchored or 
transmembrane proteins located on the plasma membrane and in the SV membrane, that are believed to overcome the energy barrier for the merger of the two bilayers, by bringing the two opposing membranes into close proximity (Jahn and Scheller, 2006). Other protein factors are also essential for this event, such as Munc proteins (Jahn, 2000) and complexins (Brose, 2008). It is clear nowadays, that the synchronous release is based on the interplay of various factors and that single components (e.g. SNAREs or synaptotagmins) are most likely not enough to drive a physiological fusion recation. The fusion ultimately releases the contents of the SV into the synaptic cleft and leads to the collapse of the SV membrane into the plasma membrane (an exception would be the "kiss and run" mode

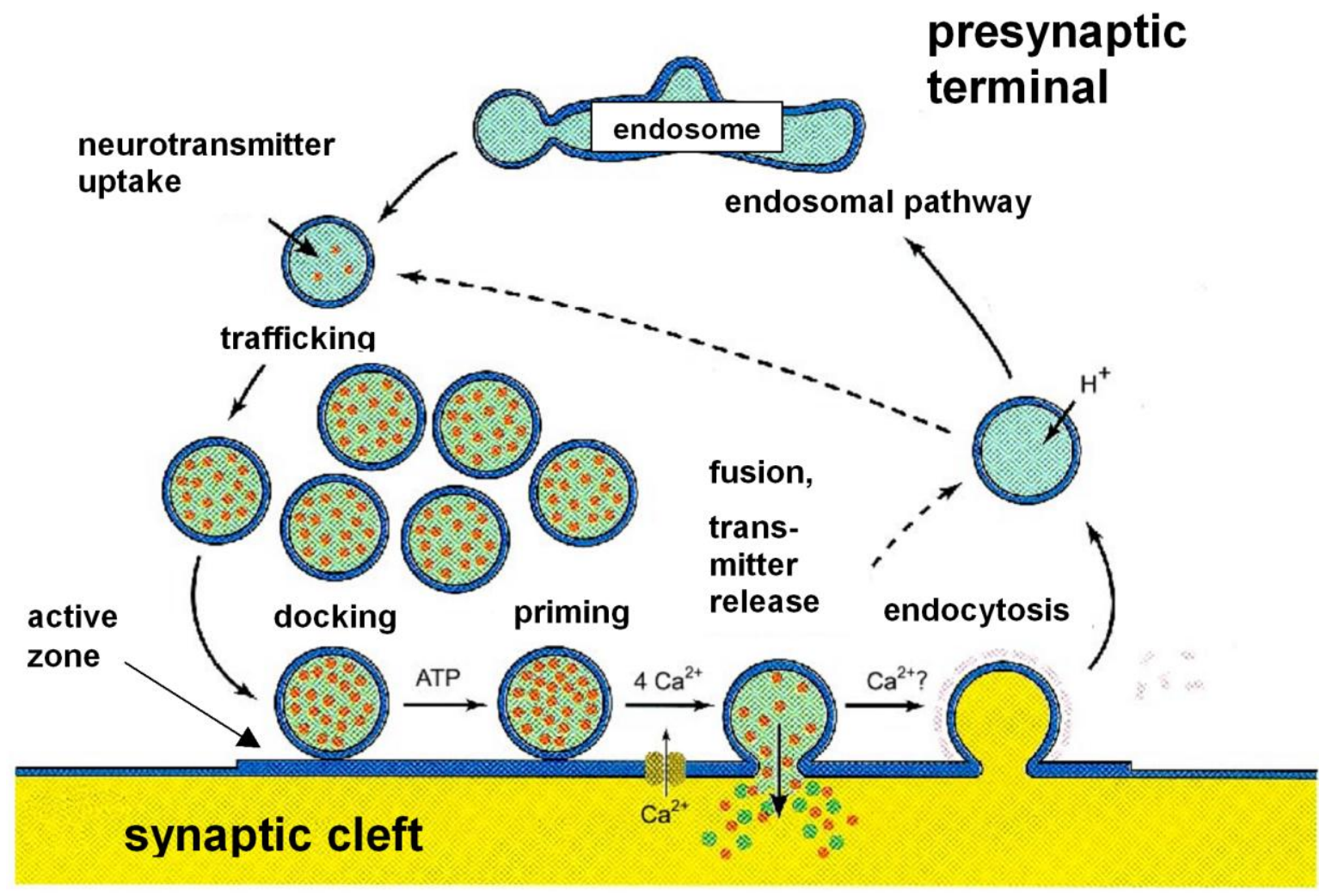

Figure 1.1

The synaptic vesicle cycle

Schematic representation of the most important steps of neurotransmitter release in the presynaptic terminal. After docking and priming at the active zone on the presynaptic plasma membrane, synaptic vesicles fuse upon $\mathrm{Ca}^{2+}$ triggering and release the transmitter. Then the vesicles are endocytosed and enter either the connected endosomal pathway or recycle directly. During the reccling the vesicles are recharged with transmitter and again competent for another round of fusion. The endosomes are involved in synaptic vesicle protein sorting and maturation of synaptic vesicles but synaptic vesicles can recycle also directly. Slightly modified from Südhof, 2004.

of transmitter release (Smith et al., 2008)). Since the essential neuronal SNARE proteins (synaptobrevin on the SV membrane and syntaxin and SNAP-25 on the plasma membrane) 
form a tight helical bundle upon fusion (Sutton et al., 1998) the first recycling event in the SV cycle has to take place - the disassembly of the SNARE complex. Releasing the constituents of the SNARE-complex requires the consumption of ATP by the AAA+ ATPase NSF ( $N$-ethylmaleimide sensitive factor), together with $\alpha$-SNAPs, essential cofactors for the disassembly reaction (Sudhof, 2004). The free SNAREs are then redirected to their corresponding membrane and contribute again to a fusion event.

This marks the second major necessity in the SV cycle: The clustering of the essential SVcomponents at the plasma membrane after the collapse of the SV membrane, and the budding of a newly formed vesicle. The recognition of the SV components is based on certain sorting signals in the SV constituents, and involves repector proteins that cluster the SV residents. Clathrin-mediated endocytosis requires adaptor proteins (such as AP-2) that recruit membrane binding proteins that induce membrane curvature like endophilins and further interact with proteins that are specialized on membrane scission (the GTPase dynamin) (Doherty and McMahon, 2009; Slepnev and De Camilli, 2000). The recruitment of SV residents into the area of membrane budding and the final budding event of the invaginated membrane area are taking place in very short time (in the order of seconds) which is crucial for synaptic plasticity. A sorting signal that is common to all SV proteins is missing, making it currently difficult to explain how the composition of SVs is ensured.

After the budding of the recycled vesicle from the plasma membrane, its interior is separated from the extracellular space again and the third major event of the SV cycle takes place: The refilling of the SV with neurotransmitter. The ATP-dependent filling of SVs with neurotransmitter is the central theme of this work and will be introduced in following chapters in detail.

The recharged vesicle will then be able to undergo another round of transmitter release. For this it has to be in close contact with the plasma membrane. Various proteins are involved in this targeting such as rab-proteins (small GTPases) (Zerial and McBride, 2001). The vesicle will then be docked and primed which is mediated by proteins such as munc-13 (Rizo and Rosenmund, 2008).

Although not part of the SV-cycle itself it should be noted that the released transmitters are also recycled (Edwards, 2007). Some transmitters like acetylcholine will be enzymatically inactivated in the synaptic cleft to ensure a short pulse duration, others like glutamate or dopamine will be taken up at high speed by $\mathrm{Na}^{+}$-driven plasma membrane transporters. 
The reuptake of glutamate involves glia cells (Danbolt, 2001) while for example monoamines are taken up again by neurons. Failures in the reuptake of transmitters result in pathological conditions and can occur after ischemia (i.e. oxygen deprivation). Therefore plasma membrane transporters provide another level for the regulation of synaptic transmission since they have impact on the transmitter concentration in the synaptic cleft and are therefore often the target of neuropharmacological drugs besides the transmitter receptors on the postsynaptic membrane.

\subsection{The major components of synaptic vesicles}

To understand the essence of neurotransmission, a detailed picture of the key organelle of this process is crucial. SV proteins comprise about $5 \%$ of the total brain protein (Sudhof (ed.), 2003), and exist in huge numbers of about 100-500 per synaptic terminal. Due to their homogeneity they can be isolated in relatively large amounts and also be subjected to chromatographic methods (Giompres et al., 1981; Hell et al., 1994) that yield material with very high purity. This laid the ground for a detailed description of all the major components of SVs, including not only the proteins but also the lipids. In an effort that took decades for completion, a detailed picture of this organelle was reconstructed (Takamori et al., 2006). The image that was drawn from all the information on SVs is the most detailed picture to date of a membraneous organelle (Fig. 1.2). It provides a clear framework and resource for the understanding of neurotransmission and trafficking in the secretory pathway.

The protein components of SVs can be divided into three major functional groups. First, the SVs are equipped with trafficking components that ensure the proper localization and recycling of SVs. These are rabs, synapsins and SNAREs. Synapsins are involved in the trafficking of SV precursors along the axon to the synaptic terminal (Greengard et al., 1994).

The second group of essential SV proteins is involved in the fusion of the SV membrane with the plasma membrane upon a trigger signal which is $\mathrm{Ca}^{2+}$. The fusion of the bilayers releases the transmitters stored in SVs into the synaptic cleft. SVs bear about 70 synaptobrevin molecules that form the SNARE-complex with the plasma membrane 


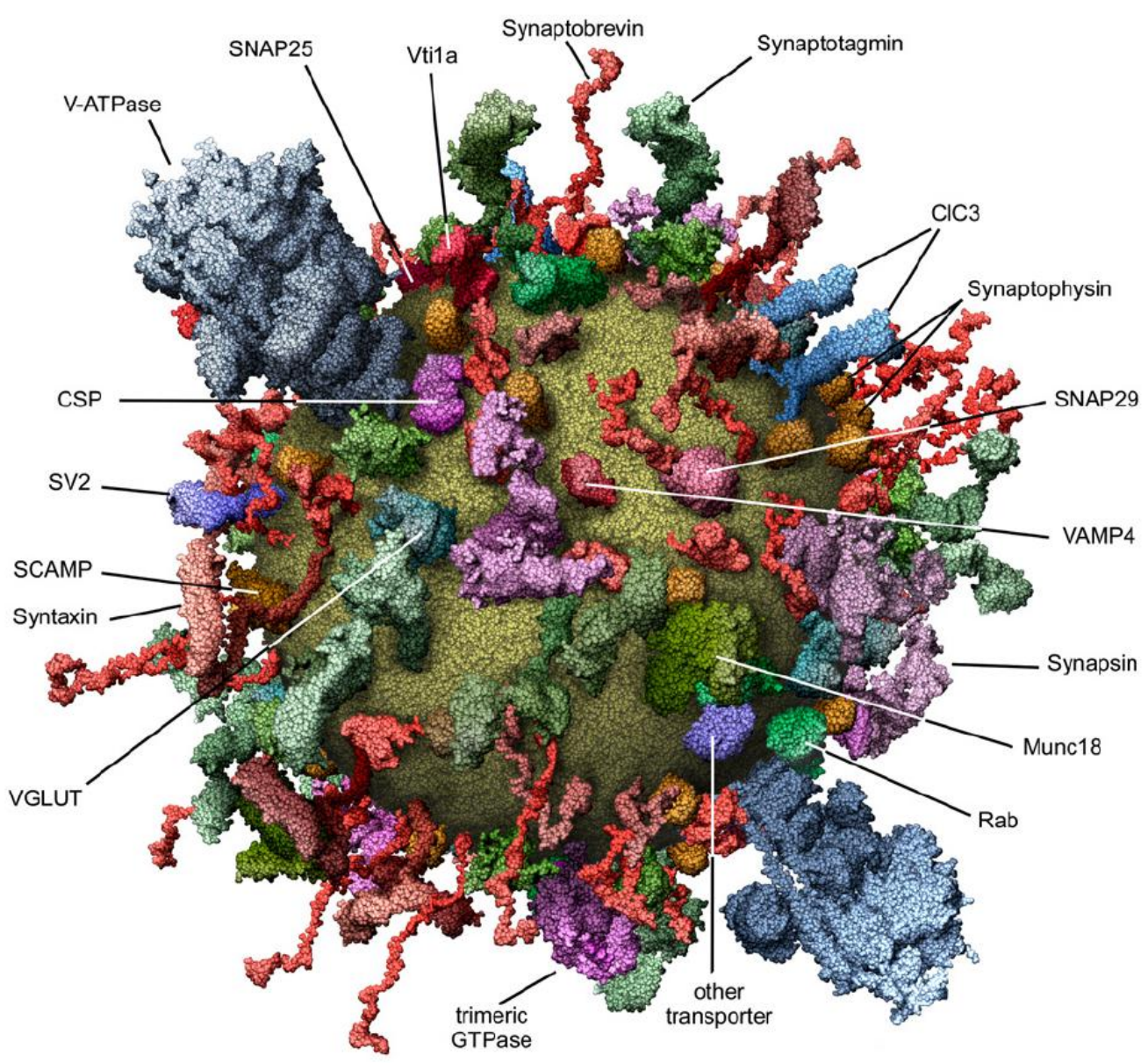

\section{Figure 1.2}

A molecular model of an average synaptic vesicle

Scaled, schematic representation based on the quantitative analysis of the major synaptic vesicle components including the lipids. The major synaptic vesicle residents are Synaptobrevin ( 70 copies), Synaptophysin ( 30-40 copies), Synaptotagmin ( 15 copies), VGLUT (for a glutamatergic vesicle : $\sim 10$ copies) and the V-ATPase ( 1-3 copies). Atomic structures were taken whenever available. Other components are processed by molecular modelling to present a likely fold. The V-ATPase has a height of $\sim 20 \mathrm{~nm}$. Taken from Takamori et al., 2006.

SNARE proteins syntaxin-1 and SNAP-25 (synaptosome-associated protein of $25 \mathrm{kDa}$ ). For this, the largely unfolded SNAREs zipper up from the $\mathrm{N}$ - to the $\mathrm{C}$-terminus and form a tight helical bundle that brings the two bilayers into close proximity and is thought to provide the force to overcome the energy barrier of merging the bilayers (Pobbati et al., 2006). Another abundant protein in this context is synaptotagmin, the $\mathrm{Ca}^{2+}$ sensor for synchronous release of transmitter (Fernandez-Chacon et al., 2001). Synaptotagmin contains two $\mathrm{Ca}^{2+}$ binding $\mathrm{C} 2$-domains that lead to an interaction with the bilayer via acidic phospholipid headgroups upon $\mathrm{Ca}^{2+}$ entry. This interaction brings the two opposing 
membranes closer to each other, might induce bending of the membrane and finally causes the fusion of the membranes (Martens and McMahon, 2008; Rizo and Rosenmund, 2008). It is however currently not clear whether synaptotagmin acts strictly downstream of the SNAREs. Complexins, small cytosolic proteins that bind tightly to the SNARE complex are additional important factors for synchronized transmitter release (Brose, 2008; Sudhof and Rothman, 2009). The fusion reaction itself is a matter of intense research, doubtlessly however it is based on SNAREs, synaptotagmins and complexins and requires the presence of certain lipids, such as phosphatidylserine and phosphatidyl-phosphoinositides (PIPs) (Di

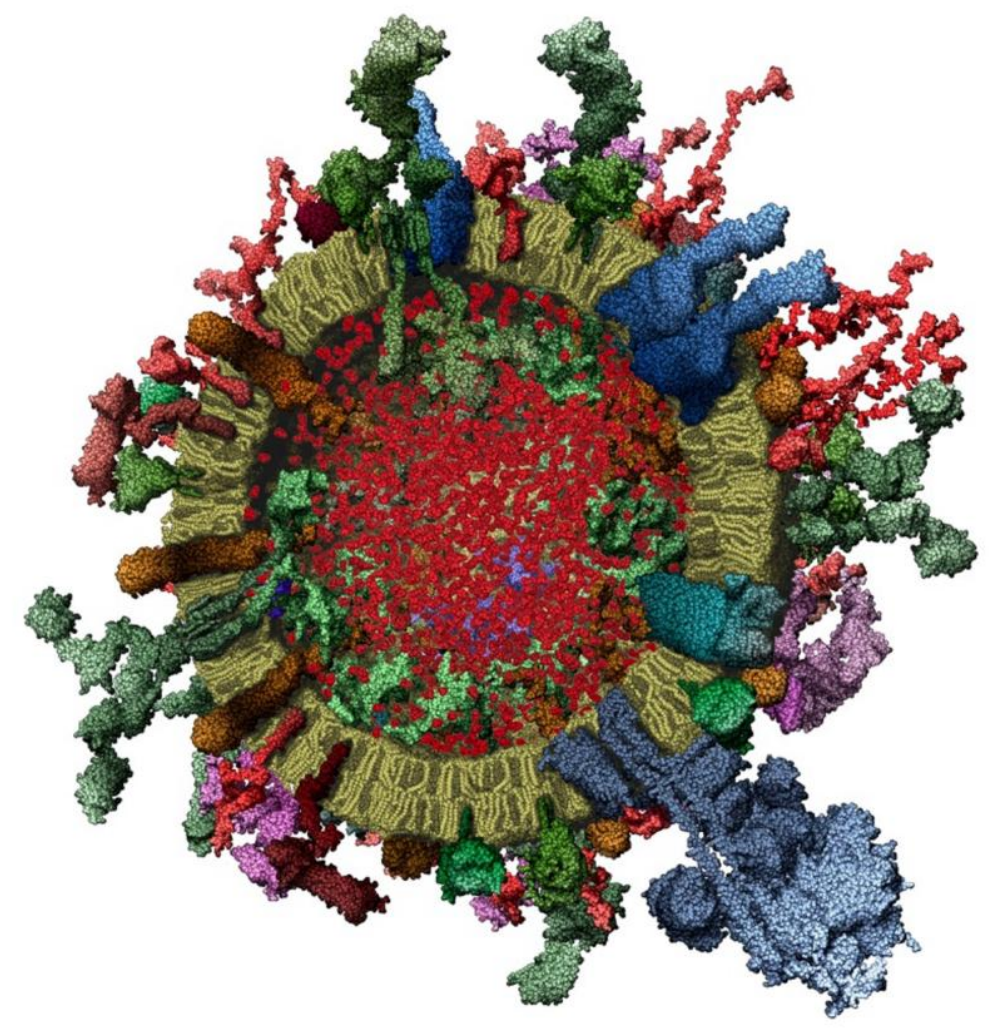

Figure 1.3

A charged synaptic vesicle cut into half

Scaled, schematic representation (see Fig. 1.2). The concentration of glutamate in the synaptic vesicles is about $100 \mathrm{mM}$. This corresponds to 1500 glutamate molecules shown as red ball models in the lumen. The luminal domains of Synaptophysin (brown) and Synaptotagmin (greygreen) extend far into the lumen. Phospholipids are shown in beige and cholesterol in dark brown. The thickness of the bilayer is about $4-5 \mathrm{~nm}$. See figure 1.2 for protein names.Taken from Takamori et al., 2006.

Paolo and De Camilli, 2006) to be carried out with precision in the observed millisecond timescale upon $\mathrm{Ca}^{2+}$ triggering.

The third group of SV residents is responsible for charging the SV with transmitters. A fully charged SV, cut into half to show its contents can be seen in figure 1.3. The energy for the transport of solutes is provided by a vacuolar-type ATPase (V-ATPase). The VATPase is present on almost all intracellular membranes and introduces a protonelectrochemical gradient $\left(\Delta \mu_{\mathrm{H}+}\right)$ that is required for the transport of solutes. The V-ATPase is present in relatively low copy numbers on SVs (1-3 copies) (Takamori et al., 2006). 
Secondary active transporters on the SV then use the electrochemical gradient to translocate the transmitter into the lumen of the vesicle and concentrate it relative to the cytosol. Importantly, the specific neurotransmitter transporters also define the phenotype of the neuron - whether it releases the excitatory transmitter glutamate or for example the inhibitory transmitter $\gamma$-aminobutyric acid (GABA). Since a transmitter like glutamate is ubiquitously present in relative high concentrations of $\sim 10 \mathrm{mM}$ in the cytosol of all neurons, the expression of vesicular glutamate transporters suffices to define a glutamatergic neuron (Takamori et al., 2000a). Other transmitters can require additional enzymes that enrich the transmitter substance in the cytosol, to provide substrate for the translocators. The number of vesicular glutamate transporters per SV has been estimated to be 10 (Takamori et al., 2006), similar numbers should account also for other vesicular neurotransmitter transporters. It is under discussion whether the number of transporters defines the total charge of transmitter per SV, the quantal size, and therefore influences synaptic transmission. For example a synapse could be strengthened by upregulation of a specific transporter, resulting in potentiation. However this idea does not seem to account for all transporter types to the same extent and the available data is still contradictory (Takamori, 2006). SVs have been shown to recycle also in the absence of any transmitter in their lumen (Wojcik et al., 2004) or when the V-ATPase is inhibited (Ikeda and Bekkers, 2009). Another important factor in the transmitter charging machinery of SVs would be ion channels (see ClC-3 in Fig. 1.2). These channels, as discussed later in detail, would influence the electrochemical gradient $\Delta \mu_{\mathrm{H}+}$, generated by the V-ATPase. However, the presence of ion channels, although postulated for a long time, has remained controversial.

The SVs also harbor a number of proteins with unclear function, sometimes even without any indication for its function. For instance, the first integral membrane protein on SVs to be identified, synaptophysin (Sudhof et al., 1987), is still of unknown function. This appears even more surprising taking its high copy number per vesicle ( $30-40)$ into account. It is widely used as a marker since it has a very sharply defined distribution on SVs. Closely related proteins of this relatively small membrane protein, like synaptogyrin appear to affect synaptic plasticity, as judged from knock out (KO) studies (Janz et al., 1999 b). They are present in much lower numbers ( 1-3) and are probably not ubiquitously present in all terminals like synaptophysin. Another long known SV resident is SV2 (Feany et al., 1992). This large transporter-like membrane protein is highly glycosylated and 
present with $\sim 2$ copies per SV. It has been shown to be the receptor for botulinum toxin A (Dong et al., 2006). SV2 appears in three isoforms (SV2A, SV2B, SV2C). The deletion of SV2A as well as the SV2A / SV2B double-KO leads to severe postnatal seizures and lethality indicating a critical function in the synapse (Janz et al., 1999a). It has been proposed to be a neurotransmitter transporter (Feany et al., 1992) or a cation exchanger (i.e. $\mathrm{Ca}^{2+}$ ) (Janz et al., 1999a). However, yet no clear evidence is supporting these ideas. If it acted as a cation translocator it would surely be important with respect to transmitter loading. SV2 has been recently shown to be the target of the major antiepileptic drug levetiracetam (Lynch et al., 2004), supporting an important role in neurotransmission.

SVs also harbor also proteins that are mainly present in endosomes such as certain rabisoforms like rab5 and endosomal SNAREs like Vtila or VAMP4. Some of these detected components might arise from contaminations, but they also reflect the fact that SVs are connected to endosomes which are known to be involved in SV recycling (Rizzoli et al., 2006). However the absolute quantities of these factors is not known and difficult to estimate. The image shown in figure 1.2 represents an average synaptic vesicle. In general, it is unclear if all of the shown protein components are equally distributed or if they are probably specific to certain terminals (except for the specific neurotransmitter transporters). Therefore a detailed analysis of SV subtypes (e.g. defined by the neurotransmitter transporter) might show that these subtypes harbor a different set of proteins.

\subsection{Loading of synaptic vesicles with neurotransmitters}

A detailed understanding of neurotransmission must include a solid concept of neurotransmitter loading into SVs besides deciphering the other stages of the synaptic vesicle cycle. This includes a biophysical coherent description of the transmitter transport and storage as well as possible regulatory mechanisms. The transmitters itself are important factors to consider in this context since their characteristics like charge, cytosolic concentration, solubility and membrane permeability largely define the potential load and the translocation itself. Nevertheless, like the concentrative import of other solutes into vacuolar membranes, all classical neurotransmitters are translocated by the ATP- 
consuming activity of the V-ATPase and thus their transporters share the electrochemical gradient as the common energy source to concentrate transmitters in SVs.

\subsubsection{Driving the uptake: Generation of a proton-electrochemical gradient.}

Intracellular compartments such as endosomes and lysosomes, but also secretory granules like chromaffin granules or SVs, are energized by the proton translocating activity of the V-ATPase (Cidon et al., 1983; Moriyama and Nelson, 1987; Schneider, 1987; Xie and Stone, 1986). This stands in contrast to secondary transport at the plasma membrane which is driven by a $\mathrm{Na}^{+}$gradient across the cell border, that is generated by the elecrogenic activity of the $\mathrm{Na}^{+}-\mathrm{K}^{+}$-ATPase. The V-ATPase is a large protein complex $(\sim 500 \mathrm{kDa})$ and consists of several subunits, some in multiple copies (Inoue et al., 2005). The cytosolic outer V1 domain consists of 8 subunits (A-H) with the stoichiometry of $\mathrm{A}_{3} \mathrm{~B}_{3} \mathrm{C}_{1} \mathrm{D}_{1} \mathrm{E}_{1} \mathrm{~F}_{\mathrm{x}} \mathrm{G}_{2} \mathrm{H}_{\mathrm{x}}$ (the copy number of the $\mathrm{F}$ and $\mathrm{H}$ subunits is not yet determined). The $\mathrm{G}$ subunits are associated with the stalk (subunit a) that connects the V1 part with the bilayer. The ATP hydrolysis is taking part in the $\mathrm{A}_{3} \mathrm{~B}_{3}$ domain. A central stalk made up of the D subunit with the associated $\mathrm{E}$ and $\mathrm{F}$ subunits connects the V1 part to the membrane embedded, integral V0 domain that consist of the above mentioned a subunit (pp116), d subunit and three similar c subunits (c, c' and c") with the stoichiometry $\mathrm{a}_{1} \mathrm{~d}_{1}\left(\mathrm{c}, \mathrm{c}^{\prime}, \mathrm{c}^{\prime \prime}\right)_{6}$. The c subunits comprise the proteolipid ring that harbors the channel for proton translocation. The central stalk is brought to stepwise rotation by catalytic steps of ATP hydrolysis in the A and B subunits. This rotating stalk is linked to the proteolipid ring which is then rotating in the membrane relative to the a-subunit and thereby pumps protons into the lumen $\left(3 \mathrm{H}^{+}\right.$ upon hydrolysis of one ATP). Structurally it resembles the ATP-Synthase of mitochondria where $\Delta \mu \mathrm{H}^{+}$, generated by the electron transport of the respiratory chain, is used to synthesize ATP from ADP and $\mathrm{P}_{\mathrm{i}}$. For the V-ATPase, ATP synthesis has been demonstrated by the coexpression of the V-PPase (a proton pump that hydrolyzes pyrophosphate $\left(\mathrm{PP}_{\mathrm{i}}\right)$ to pump protons) in yeast vacuolar membranes (Hirata et al., 2000). The V-PPase provided the proton electrochemical gradient for ATP synthesis by the VATPase. However, normally the equilibrium of ATP / ADP $+\mathrm{P}_{\mathrm{i}}$ in the cytosol would prevent the synthesis of ATP, despite the fact that the V-ATPase itself is providing $\Delta \mu_{\mathrm{H}+}$. 
In case of the mitochondrial ATP-Synthase the ATP / ADP ratio is shifted towards ADP by ATP/ADP-translocators on the inner membrane of mitochondria and $\Delta \mu_{\mathrm{H}+}$ is generated by the oxidative respiration.

Due to the hydrolysis of ATP, the V-ATPase builds up a proton electrochemical gradient $\left(\Delta \mu \mathrm{H}^{+}\right) . \Delta \mu \mathrm{H}^{+}$is then utilized for transport processes across the membrane and consists of two components, the membrane potential $(\Delta \Psi)$ and the $\mathrm{pH}$-gradient $(\Delta \mathrm{pH})$. The ratio of these two components is modulated by the permeability of the membrane for certain ions by leaks or channels and profoundly influences the interior of vesicles, such as their $\mathrm{pH}$ and thus enzymatic activities and / or transport of solutes. The V-ATPase is an electrogenic enzyme which means that in the absence of conductivities other than for protons $\Delta \mu \mathrm{H}^{+}$is comprised entirely of $\Delta \psi$. However in the presence of channels that allow for counterion or pairion movement this current is shunted and certain isoforms of the V-ATPase can establish pH values as low as 2-3 in lemon fruit vacuoles (Muller et al., 1996). Regarding the transport of solutes, both $\Delta \Psi$ and $\Delta \mathrm{pH}$ can be utilized to energize the translocation process. For instance, biochemical investigations on isolated SVs revealed that the preference for either component depends on the specific transport system for different classical neurotransmitters (Edwards, 2007; Maycox et al., 1990b).

\subsubsection{Glutamate is the major excitatory transmitter in the vertebrate brain and is translocated by vesicular glutamate transporters (VGLUTs)}

The amino acid glutamate is a widely used neurotransmitter in animals. In arthropods it serves as transmitter at the neuromuscular junction and some specific peripheral and central synapses (Daniels et al., 2008; Gerschenfeld, 1973) and in vertebrates it is the major excitatory transmitter in the CNS where it excites virtually all neurons (Jahr and Lester, 1992). The ATP-dependent uptake of glutamate into isolated SVs has been first described over 20 years ago (Disbrow et al., 1982). Later studies showed that the ATP consumption and glutamate transport are separate units (Lewis and Ueda, 1998; Maycox et al., 1990a; Moriyama et al., 1991) .

Today it is known that the transport into SVs is mediated by vesicular glutamate transporters (VGLUTs) which are members of Solute Carrier family 17 (SLC17). They are 
low affinity transporters with a $K_{M}$ of 1-2 mM (Naito and Ueda, 1985; Wolosker et al., 1996). The VGLUTs remained molecularly unidentified for a relatively long time despite the wide distribution of glutamatergic neurons. In fact, they were the last transporters for classical small neurotransmitters to be identified. The first isoform (VGLUT1) was initially described as brain-specific Na+-dependent phosphate transporter (BNPI). The BNPI sequence was found to be upregulated in neurons by subtoxic concentrations of N-methylD-aspartate (NMDA) (Ni et al., 1994). When the cRNA was injected into Xenopus oocytes, BNPI conferred $\mathrm{Na}^{+}$dependent uptake of inorganic phosphate $\left(\mathrm{P}_{\mathrm{i}}\right)$ (Ni et al., 1994). It was suggested to play a role in maintenance of energy stores (Glinn et al., 1998). But its selective expression in neurons ( $\mathrm{Ni}$ et al., 1995) suggested a role more specifically linked to neurotransmission. The BNPI orthologue in C. elegans, eat-4, shows defects in glutamatergic transmission when mutated (Avery, 1993). In this mutant, the neurons still respond to iontophoretically applied glutamate which suggested that the defects of the eat4 mutation are presynaptic (Dent et al., 1997). Furthermore, BNPI localizes to excitatory nerve terminals and more specifically to SVs (Bellocchio et al., 1998). This suggested that BNPI might have a function different from $\mathrm{P}_{\mathrm{i}}$ uptake. It was then found that BNPI, heterologously expressed in secretory vesicles, transports glutamate (Bellocchio et al., 2000; Takamori et al., 2000a). Importantly, the transport showed the same characteristics as described already for isolated vesicles, such as the dependence on $\mathrm{Cl}^{-}$(Bellocchio et al., 2000) or the sensitivity towards the dye Evan's Blue (EB) (Bellocchio et al., 2000; Takamori et al., 2000a). Furthermore, it was highly specific for L-glutamate, but not aspartate and was not depending on a $\mathrm{Na}^{+}$gradient like the glutamate transporters on the plasma membranes (excitatory amino acid transporters : EAATs). A very elegant proof for the function of BNPI as a vesicular glutamate transporter came from an experiment where BNPI was overexpressed in GABAergic neurons, which resulted in co-release of glutamate (Takamori et al., 2000a). The name was then changed from BNPI to VGLUT. It is still under debate whether VGLUTs might function also as $\mathrm{Na}^{+}$dependent $\mathrm{P}_{\mathrm{i}}$ transporters. A recent study supports such a function (Juge et al., 2006), while other authors argue against this (Edwards, 2007) . 


\subsubsection{The VGLUT isoforms and their distribution in the mammalian brain}

VGLUT1 (also known as Slc17a7) localizes to a part of excitatory neurons of the adult brain, mostly in the cortex, hippocampus, and cerebellar cortex (granule cells). A newly discovered $\mathrm{Na}^{+}$dependent $\mathrm{P}_{\mathrm{i}}$ transporter showed strong sequence similarity to VGLUT1 (Aihara et al., 2000). This differentiation-associated $\mathrm{Na}^{+}$-dependent $\mathrm{P}_{\mathrm{i}}$ transporter (DNPI) was then found to translocate glutamate with transport characteristics, and kinetics that are very similar to VGLUT1 when heterologously expressed on secretory vesicles (Bai et al., 2001; Fremeau et al., 2001; Herzog et al., 2001; Takamori et al., 2001). DNPI was then renamed to VGLUT2 (Slc17a6) and shows an almost exclusive expression pattern from VGLUT1. In situ hybridization revealed that it expresses in neurons were VGLUT1 is not expressed (Fremeau et al., 2001) confirmed by immunocytochemical methods. These brain regions include the thalamus and the brainstem. A third isoform in mammals, VGLUT3 (Slc17a8) (Fremeau et al., 2002; Schafer et al., 2002; Takamori et al., 2002) shows an unusual distribution pattern. It expresses in neurons that are not traditionally associated with glutamate release, like serotoninergic neurons in dorsal and medial raphe nuclei, cholinergic interneurons in the striatum and subsets of GABAergic neurons in the hippocampus and the cortex (Fremeau et al., 2002; Gras et al., 2002; Schafer et al., 2002). The knock-out of the VGLUT3 gene revealed a role in glutamatergic transmission in the inner ear, since the deletion of VGLUT3 leads to deafness (Seal et al., 2008). In cholinergic neurons, the coexpression of VGLUT3 enhances the uptake of acetylcholine (Gras et al., 2008). It is still unclear what might be the biochemical background for such an enhancement but one could speculate that glutamate serves as a pairion to keep electroneutrality or increases $\Delta \mathrm{pH}$ which is the major driving force for vesicular acetylcholine transport..

The deletion of the VGLUT1 gene (Fremeau et al., 2004; Wojcik et al., 2004) leads to a loss of glutamate release in neurons that express VGLUT1 in adulthood. However, during development, these neurons transiently also express VGLUT2 in the hippocampus and cerebellum which leads to residual, VGLUT1-independent release of glutamate (Fremeau et al., 2004; Wojcik et al., 2004). This release shows the same baseline characteristics as in wild type cells, although it depresses faster. Some authors suggested that this transient 
VGLUT2 expression is restricted to distinct synapses (Fremeau et al., 2004), others proposed that the two isoforms transiently colocalize to SVs and not to distinct synapses (Herzog et al., 2006; Wojcik et al., 2004). VGLUT1 expression is already present at birth, but reaches its maximum level after about 3 weeks in mice. The deletion of the gene is surprisingly not lethal and despite a relatively high mortality before adulthood, mice can survive up to several months without synaptic activity in excitatory terminals in large areas of the cortex and hippocampus. In contrast, the VGLUT2 deletion is lethal at birth (Moechars et al., 2006). Since the brainstem is responsible for respiration, and excitatory terminals in this region mainly expresses VGLUT2, this is an expected phenotype.

VGLUT1, 2 and 3 are highly homologous, except for the C-terminal tail which contains a proline rich domain in VGLUT1. It has been shown that this proline-rich domain is a recognition signal for the endophilins, proteins that are involved in SV endocytosis which confers different rates of recycling to VGLUT1 and VGLUT2 (Voglmaier et al., 2006). Consistent with this finding is that the VGLUT1 KO shows a lower number of SVs per terminal compared to the wild type (Fremeau et al., 2004). However, it has been demonstrated that VGLUT1 KO neurons are capable of normal SV-release and recycling (Wojcik et al., 2004).

Though not isoforms of VGLUTs, additional vesicular neurotransmitter transporters of the SLC17 family have been recently described. The gene Slc17a9 has been identified as a vesicular nucleotide transporter (VNUT) (Sawada et al., 2008), that has been postulated for a long time and is responsible for the accumulation of ATP in the peripheral and central nervous system where ATP can act as a transmitter (Pankratov et al., 2006). The transport has been described to be driven mainly by $\Delta \psi$, with yet unclear stoichiometry (Sawada et al., 2008). In a work originating from the same laboratory, additional roles for another Slc17 member, Sialin (Slc17a5), have been described. It has been demonstrated that Sialin is also capable of transporting L-Glutamate and L-Aspartate (Miyaji et al., 2008). Therefore the additional name VEAT (vesicular excitatory amino acid transporter) was proposed. It was shown to be expressed in pinealocytes where it acts as the major vesicular glutamate transporter. Sialin/VEAT can be distinguished from VGLUTs by its substrate specificity since VGLUTs do not translocate aspartate. On lysosomes Sialin is acting as an efflux carrier for sialic acid (Morin et al., 2004). 


\subsubsection{Structural features of VGLUTs}

To date, little is known about the structure of VGLUTs. The $\mathrm{N}$ - and C-terminal ends are known to be located to the cytoplasm consistent with the interaction with endophilins at a proline rich domain at the C-terminus (De Gois et al., 2006; Voglmaier et al., 2006). Further evidence is coming from experiments with antibodies directed against $\mathrm{N}$ - and $\mathrm{C}$ terminal peptides in the Drosophila isoform (Fei et al., 2007). Secondary strucure predictions and hydrophilicity plots suggest 10-12 transmembrane regions (TMRs). Currently 12 TMRs are the favored view since VGLUTs resemble the lactose permease, which is commonly used to model VGLUTs in structure function predictions (Almqvist et al., 2007; Takamori et al., 2006). It is likely that the complete gene arose from a gene duplication event, since the $\mathrm{N}$ - and $\mathrm{C}$-terminal halfs show almost the same secondary structure and are connected by a relatively long (presumably) cytoplasmic loop, similar to the lactose permease, a transporter for which the structure has been solved (Abramson et al., 2003). The first luminal loop is relatively long and contains a site for N-linked glycosylation. An internal $\mathrm{pH}$-sensitive GFP-fusion protein introduced within this loop did not interfere with the targeting of VGLUT1 (Voglmaier et al., 2006). The pH sensitive GFP further supported the luminal orientation of this first loop since it changes its fluorescence upon exocytosis due to $\mathrm{pH}$ changes which is only compatible with a luminal localization. Due to glycosylation the VGLUTs run with a smeary appearance in SDS-gels at a molecular weight of $60-70 \mathrm{kDa}$ depending on the isoform or ortholog. If the glycosylation is essential for the function is not known. The C-terminal loop contains a leucine-internalization signal (Voglmaier et al., 2006). Mutation of this signal sequence interferes with the localization of VGLUTs and leaves more transporters on the plasma membrane when they were heterologously expressed. The interaction with endophilins via the proline rich domain of VGLUT1 has been shown to influence synaptic physiology, by faster recycling rates. It was proposed that this is due to the recruitment to specific endocytic pathways that operate with relatively high speed (AP-2 pathway). VGLUT2 does not bear this signal sequence, therefore probably is not recycled by the AP-2 pathway of endocytosis and thus VGLUT2 terminals depress faster (Fremeau et al., 2004; Voglmaier et al., 2006). This signal sequence determines therefore an important functional difference 
between VGLUT1 and 2 which demonstrates that the VGLUTs are involved in synaptic plasticity.

\subsubsection{VGLUTs and quantal size}

Influence of the expression of vesicular transporters on the quantal size has been demonstrated for monoaminergic transmission by vesicular monoamine transporters (VMATs) (Fon et al., 1997; Pothos et al., 2000) (but see also (Bruns et al., 2000)) and for cholinergic transmission by the vesicular acetylcholine transporter (VAchT) (Song et al., 1997). VGLUTs have been proposed to influence quantal size and thereby synaptic plasticity (Takamori, 2006; Wilson et al., 2005). So far synaptic potentiation has been largely addressed to postsynaptic changes so that a presynaptic influence by the VGLUTs of this important parameter would confer another level of regulation in neurotransmission. This idea has been supported by overexpression of VGLUT1 in hippocampal neurons. For this, VGLUT1 was overexpressed by the Semliki-Forest-Virus in wild type glutamatergic autaptic neurons (Wojcik et al., 2004). Indeed, VGLUT1 overexpressing neurons showed larger miniature excitatory postsynaptic currents (miniature EPSCs) than wild type glutamatergic neurons. When neurons, heterozygous for VGLUT1, and thus expressing about half the amount of transporter protein, were analyzed, very little or no obvious changes in quantal size were described (Fremeau et al., 2004; Wojcik et al., 2004). However, a miniature EPSC analysis of $\operatorname{Vglut}^{+-}$thalamic neurons showed a slight reduction of the quantal size (Moechars et al., 2006). This is consistent with behavioral deficits in $\mathrm{Vglut}^{+/-}$mice observed in the same study, showing that the copy number has impact on the animal physiology. Recently behavioral deficits, such as depressive-like behavior have been also reported for Vglut $^{+/-}$mice (Garcia-Garcia et al., 2009; Tordera et al., 2007). It could thus be that the changes in quantal size are too subtle to be reliably detected in miniature EPSCs, due to the low signal to noise ratio that limits these measurements.

A series of investigations at the neuromuscular junction of Drosophila melanogaster, where a VGLUT ortholog is expressed (DVGLUT) do not support an immediate effect of the transporter number per vesicle on the quantal size. A mutant strain of Drosophila that 
expresses extremely low levels of DVGLUT did not show smaller excitatory postsynaptic potentials (EPSPs), leading to the conclusion that even a single transporter suffices to charge a vesicle fully (Daniels et al., 2006). When DVGLUT was overexpressed in the neuromuscular junction an increase of quantal size was observed. However, the quantal charge remained constant suggesting compensatory mechanisms, presumably of postsynaptic origin (Daniels et al., 2004). Furthermore it was suggested that this increase in quantal size is due to changes in the volume of SVs as judged by electron microscopic studies (Daniels et al., 2004).

It is therefore not yet clear if VGLUTs confer changes to the quantal size by their copy number on SVs since the data is still controversial. The transport and storage of glutamate on SVs should also be important factors in this context (Edwards, 2007). For instance if glutamate storage would be leaky by a nonspecific leak, a higher number of transporters might have influence on the charge of the vesicle. If such a leak was absent, and glutamate was stored at equlibrium, the ionic coupling of VGLUTs together with the $\mathrm{H}^{+}$ electrochemical driving force should dictate the maximal filling of a SV (equilibrium model). In the set-point model a vesicle would also fill to a fixed set point, but only indirectly related to the transport mechanism. Increased transporter expression would increase the content, however the maximal load would be probably less than expected from the increased transporter expression. Therefore a physical factor such as vesicle size could limit the total load, and a higher number of VGLUT molecules is probably only affecting the loading kinetics. An important factor in this context would be that lipid membranes are inelastic and therefore the osmotic pressure in the vesicle must also be limiting (Maycox et al., 1990b). Therefore the vesicle size appears as a likely determinant of the quantal size together with the tightness of the membrane for glutamate. While the leakage of glutamate from SVs in the absence of a driving force has been reported (Burger et al., 1989), some recent studies showed that the blockade by bafilomycin $\mathrm{A}_{1}$ (a specific inhibitor of the $\mathrm{V}$ ATPase) did not induce glutamate leakage in physiological timescales (Ikeda and Bekkers, 2009). Further investigations on the glutamate translocation will be helpful to answer such questions in the light of a probable role for the regulation of quantal size by VGLUTs. 


\subsubsection{The chloride dependence of VGLUTs: Implications for the energy require- ments of glutamate translocation}

Vesicular transport of glutamate is ATP dependent. It is however a secondary active transport that is fueled by the activity of the V-ATPase. The separation of glutamate transport from the ATP hydrolysis has been shown already before the identification of VGLUTs by reconstitution of SV proteins into liposomes. When such liposomes were energized by a proton pump that is pharmacologically distinguishable from the V-ATPase, glutamate uptake was possible (Maycox et al., 1990a; Moriyama et al., 1991). Furthermore, glutamate uptake occurred by imposing an ATP independent electrochemical gradient on reconstituted liposomes with the ionophore valinomycin (Hell et al., 1991; Shioi and Ueda, 1990). These latter experiments also fueled the idea that the uptake of glutamate into $\mathrm{SVs}$ is driven primarily by $\Delta \Psi$, supporting previous observations (Maycox et al., 1988). However the proton pump independent uptake was relatively small and because the protonophore FCCP almost inhibited the transport, it might be that protons are involved in the translocation (Shioi and Ueda, 1990). The idea that the glutamate translocation depends mainly on $\Delta \Psi$ did arise mainly from the notion that high concentrations of $\mathrm{Cl}^{-}(10-150 \mathrm{mM})$ have an inhibitory effect on glutamate uptake and also because dissipation of $\Delta \mathrm{pH}$ increases uptake (Maycox et al., 1988). This is not the case for the uptake of monoamines and also to much lesser extent for the uptake of GABA into SVs (Maycox et al., 1990b). High $\mathrm{Cl}^{-}$concentrations in the assay medium indeed increase the $\Delta \mathrm{pH}$ component of $\Delta \mu_{\mathrm{H}+}$ on the expense of $\Delta \Psi$ as shown by fluorescence measurements with dyes that respond to vesicular acidification and transmembrane potential (Tabb et al., 1992).

A prominent feature of the vesicular glutamate transport is its biphasic dependence on extravesicular $\mathrm{Cl}^{-}$which has been characterized in biochemical investigations of native SVs (Naito and Ueda, 1985) and in membranes containing heterologous VGLUTs (Bai et al., 2001; Bellocchio et al., 2000; Fremeau et al., 2002; Fremeau et al., 2001; Varoqui et al., 2002). The transport activity is very low in the absence of external $\mathrm{Cl}^{-}$, maximal at low millimolar concentrations of $\mathrm{Cl}^{-}(2-4 \mathrm{mM})$ and again declining with rising $\mathrm{Cl}^{-}$ concentrations of $10-100 \mathrm{mM}$ as shown in figure 1.4. While the decrease in glutamate uptake under conditions of high concentrations of extravesicular $\mathrm{Cl}^{-}$has been attributed to 
the increase of $\Delta \mathrm{pH}$ due to a $\mathrm{Cl}^{-}$channel on SVs (Maycox et al., 1988), the very low uptake in the absence of $\mathrm{Cl}^{-}$, although $\Delta \Psi$ is maximal under these conditions, remained largely unexplainable. The transport of glutamate was therefore believed to be activated in the presence of low millimolar concentrations of $\mathrm{Cl}^{-}$by regulatory binding of this ion to the transporter itself (Hartinger and Jahn, 1993). This was further supported by the fact that dissipation of the $\mathrm{pH}$ gradient by nigericin or $\left(\mathrm{NH}_{4}\right)_{2} \mathrm{SO}_{4}$ increased the uptake of glutamate

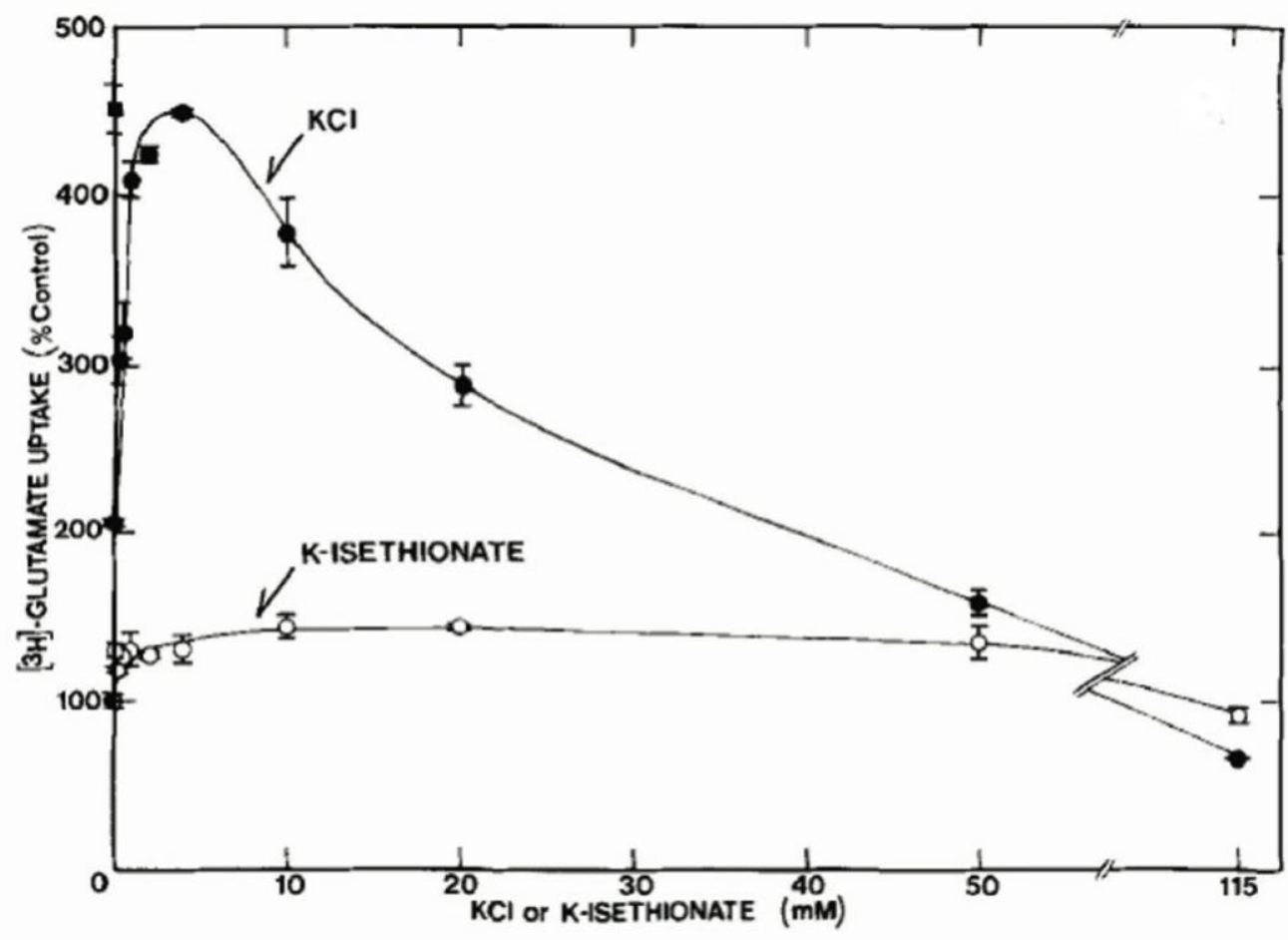

\section{$\Delta \Psi$ \\ $\Delta \mathrm{pH}$}

\section{Figure 1.4}

The biphasic dependence on extravesicular $\mathrm{Cl}$ of glutamate uptake in synaptic vesicles First description of the typical biphasic response of glutamate uptake into synaptic vesicles on the $\mathrm{Cl}^{-}$concentration in the assay medium. The data is scaled to uptake in the absence of extravesicular anions (100\%). Isethionate, a bulky anion, does not enhance the uptake significantly. $\mathrm{Cl}^{-}$enhances the uptake $\sim 4.5$-fold at low millimolar concentrations and attenuates uptake if used at higher concentrations. Modified from Naito et al., 1985

at low millimolar $\mathrm{Cl}^{-}$concentrations, which speaks against the establishement of a $\mathrm{pH}$ gradient that would be required for uptake. However, this relation could not always be reproduced (Tabb et al., 1992) (and this work). Since the effect of nigericin depends on 
the $\mathrm{Cl}^{-}$concentration and also on the ratio of glutamate to $\mathrm{Cl}^{-}$(Tabb et al., 1992; Wolosker et al., 1996) there are also indications for a role of the luminal $\mathrm{pH}$ which could explain the activation of transport by low concentrations of $\mathrm{Cl}^{-}$(Naito and Ueda, 1985). The purely electophoretic transport of glutamate, albeit supported by some authors (Juge et al., 2006), can therefore not be fully explained with experimental observations.

Recently, a regulation of transmitter loading for VMATs and VGLUTs by heterotrimeric $G$ proteins has been demonstrated. Multiple heterotrimeric G proteins associate with secretory vesicles and activation of the G protein $\alpha_{0} 2$ with nonhydrolyzable forms of GTP reduces monoamine and glutamate uptake by $30-50 \%$, primarily by affecting $v_{\max }$ rather than $K_{M}$ (Brunk et al., 2006). Remarkably, $\alpha_{0} 2$ appears to alter the $\mathrm{Cl}^{-}$dependence of vesicular glutamate transport, shifting it to lower concentrations, although the mechanism remains unclear (Winter et al., 2005).

Interestingly, when VGLUT1 was heterologously expressed on secretory granules it induced a $\mathrm{Cl}^{-}$conductance in these membranes (Bellocchio et al., 2000). Although the secretory granules also possess a $\mathrm{Cl}^{-}$conductance, the expression of VGLUT1 clearly showed higher acidifcation rates. Importantly this conductance could be inhibited by glutamate, but not aspartate, supporting the idea that the observed additional conductivity is intrinsic to the transporter. These measurements are however difficult to interprete since the presence of glutamate also leads to an acidification of the vesicle interior (see this work) albeit to lower extent. Therefore the increased $\mathrm{Cl}^{-}$conductance in heterlogously VGLUT1 expressing secretory granules could be also due to a secondary effect, like enlargement of the vesicles upon VGLUT1 expression or the induction of a natively present $\mathrm{Cl}^{-}$channel. Despite some doubts, this finding is very interesting in the light of the biphasic $\mathrm{Cl}^{-}$dependence of VGLUTs, nevertheless it was not investigated further to date and thus could not be verified in an independent manner. This is mainly due to the difficulties to express VGLUT1 heterologously for experiments that use purified VGLUT1, which would greatly ease the interpretation of results in the absence of contaminating conductivities that are present in native material. $\mathrm{A}^{-}$conductance in VGLUT1 would have a major impact on the interpretation of its $\mathrm{Cl}^{-}$dependence, calling for a deeper investigation of this phenomenon. 


\subsubsection{The energy requirements of other major vesicular neurotransmitter transporters}

The major inhibitory transmitter in the vertebrate CNS is GABA. GABA is synthesized by the L-glutamatic acid decarboxylase (GAD) enzyme from glutamate in the presynapse. The transmitter is then translocated by the vesicular GABA transporter (VGAT) (Burger et al., 1991; McIntire et al., 1993; McIntire et al., 1997; Thomas-Reetz et al., 1993). The translocation of glycine (another amino acid with inhibitory function) by VGAT has recently been confirmed by mouse models (Wojcik et al., 2006), as already suggested earlier (Burger et al., 1991). VGAT is thus also named Vesicular Inhibitory Amino Acid Transporter (VIAAT) (a member of the Slc32 family). The translocation of GABA is currently believed to involve the exchange with $\mathrm{H}^{+}$however with unclear stoichiometry. VIAAT is apparently driven by both, $\Delta \psi$ and $\Delta \mathrm{pH}$ (Hell and Jahn, 1998; Hell et al., 1990). Consequently the transport is not strongly attenuated by extravesicular $\mathrm{Cl}^{-}$. GABA and glycine are zwitterionic in the cytosol and also relatively membrane permeable compared to glutamate. As a zwitterion they do not require a pair ion, an important consideration for the storage of these transmitters. GABA would exert only half the osmotic pressure on the SV membrane at the same concentration of a salt. The transport of GABA into synaptic vesicles is particularly demanding to investigate because VIAAT is a transporter with a very low affinity $\left(K_{M} \sim 5 \mathrm{mM}\right)$ and because only about $15 \%$ of purified rat SVs are positive for VIAAT (Takamori et al., 2006). VIAAT positive vesicles that have been pulled down with antibody coated beads from SV isolates, to enrich the rare vesicle type, indicated the presence of a $\mathrm{Cl}^{-}$conductance (Takamori et al., 2000b), which has to be taken into account for the understanding of GABA/glycine uptake and the role of $\Delta \psi$ and $\Delta \mathrm{pH}$ in the translocation process.

Upon release, GABA and glycine bind to postsynaptic GABA and glycine receptors. These receptors are $\mathrm{Cl}^{-}$channels that open upon ligand binding. This leads to a hyperpolarization due to $\mathrm{Cl}^{-}$influx, thus inhibiting the neuron. The reuptake of GABA and glycine is mediated by plasmamembrane glycine (GLYTs) and GABA transporters (GATs).

VIAAT shows no sequence similarity with other neurotransmitter transporters. It is characterized by an unusual membrane topology with a luminal localization of the $\mathrm{N}$ terminus (Martens et al., 2008).Another transporter with a low apparent affinity is the 
vesicular acetylcholine transporter (VAchT) $\left(K_{M} \sim 5 \mathrm{mM}\right)$ that was originally described in the electric organ of Torpedo (Parsons et al., 1993). It is mainly localized to the neuromuscular junction of vertebrates where acteylcholine is the excitatory transmitter. Only few neurons in the brain are cholinergic. VAchT depends largely on the $\Delta \mathrm{pH}$ component of the electrochemical gradient (Parsons et al., 1993) for acteylcholine transport, a postively charged transmitter. It translocates acetylcholine in exchange for 2 $\mathrm{H}^{+}$(Nguyen et al., 1998).

Glutamate (VGLUTs)

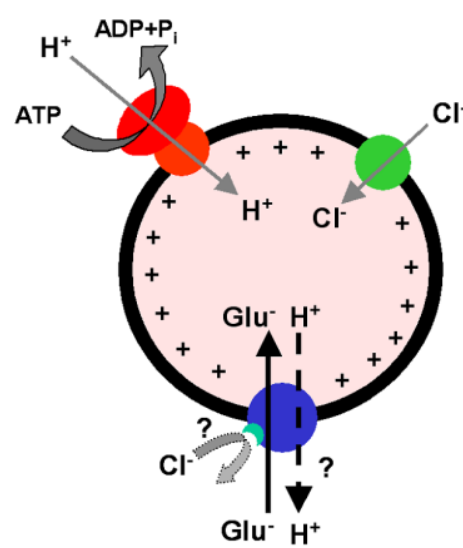

GABA (VIAAT)

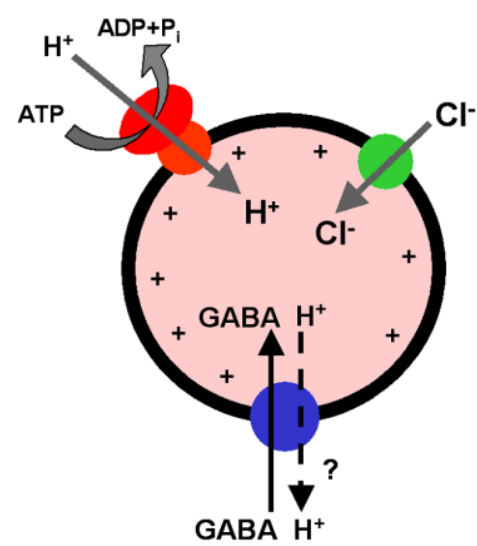

Monoamines (VMATs)

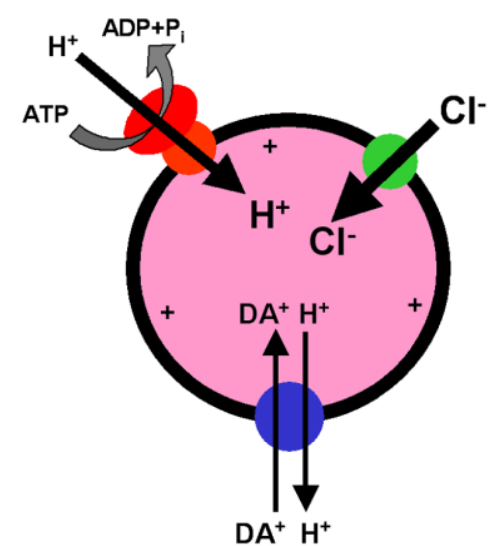

\section{Figure 1.5}

Current working models for the loading of small classical transmitters

Schematic representations of transmitter loading into SVs. Glutamate (left), translocated by VGLUTs depends largely on the $\Delta \psi$ component of $\Delta \mu_{H_{+}}$(symbolized by + ). GABA uptake (middle) is driven by $\Delta \psi$ and $\Delta \mathrm{pH}$ (symbolized by the pink background). The uptake of monoamines (here dopamine, DA) but also acetylcholine, is energized mainly by $\Delta \mathrm{pH}$ (right). A $\mathrm{Cl}^{-}$channel (green) influences the ratio of $\Delta \psi$ and $\Delta \mathrm{pH}$. The V-ATPase (red) energizes the membrane. A putative $\mathrm{Cl}^{-}$binding site on VGLUTs is shown in light green. The exchange of protons for glutmate and GABA is proposed but not proven. VMATs as well as VAchT translocate 2 protons in exchange with one monoamine or acetylcholine respectively. Additional ion conductivities (not drawn), like for cations such as $\mathrm{Na}^{+}$or $\mathrm{Ca}^{2+}$ could be present on SVs and modulate $\Delta \mu_{H_{+}}$in addition.

Transporters with high sequence similarity to VAchT, and belonging to the same family (Slc18), are the vesicular monoamine transporters (VMATs). They translocate $2 \mathrm{H}^{+}$for one positively charged amine and depend largely on the $\mathrm{pH}$ gradient (Johnson et al., 1981; Knoth et al., 1981). Substrates are biogenic monoamines like dopamine, norepinephrine and serotonine. They were originally characterized in chromaffin granules. Two isoforms of VMATs are known. VMAT1 is expressed by chromaffin and other endocrine cell lines and VMAT2 is neuronal and translocates also histamine. Chromaffin granules, filled by VMAT1 may contain very high concentrations of monoamines that probably become 
insoluble in the lumen and could cause the typical dense core (large dense core vesicles, LDCVs). The release of monoamines can be quantitatively measured by amperometry (Bruns and Jahn, 1995; Wightman et al., 1995). Such a measurement has been undertaken at Retzius neurons from the leech (Hirudo medicinalis) (Bruns et al., 2000), neurons that contain small synaptic-like vesicles (SSVs) and large dense core vesicles (LDCV). The concentration of serotonin in single synaptic vesicles from these terminals has been determined to be $270 \mathrm{mM}$ for all of the vesicle types in Retzius terminals. This indicated that the vesicle size is the major determinant for the quantal size since the mean quantal sizes of SSVs and LDCVs compared well with the average sizes of these organelles. VMATs have very high affinities for their substrates which appear only in very low free concentrations in the cytosol.

A summary for the energy requirements of the major transmitter transport systems in the vertebrate CNS is shown in figure 1.5.

\subsection{Ion permeabilities of intracellular compartments and their influence on the electrochemical gradient}

Most intracellular compartments show a $\mathrm{pH}$ value that is below the $\mathrm{pH}$ of the cytosol $(\mathrm{pH}$ 7.2-7.4) by 1-2 $\mathrm{pH}$ units (Fig. 1.6). In plant vacuoles the $\mathrm{pH}$ is often lowered by $3-4 \mathrm{pH}$ units relative to the cytoplasm. The ER and Golgi show only moderate acidification whereas lysosomes and secretory granules are the most acidic intracellular compartments in an animal cell. The acidification of organelles is a fundamental component of homeostasis. For example, alkanization of the Golgi by weak bases or V-ATPase inhibitors leads to impairment of trafficking (Presley et al., 1997), impaired processing and glycosylation of cargo proteins and lipids (Axelsson et al., 2001), missorting of some proteins (Puri et al., 2002) and morphological changes of the Golgi (Tartakoff et al., 1978). The molecular mechanisms underlying these phenomena are however poorly understood. The low $\mathrm{pH}$ in the lysosome is necessary for the degradation of proteins by proteinases that require a low $\mathrm{pH}$ for their catalytic activity (such as aspartyl proteases) and thus this low $\mathrm{pH}$ serves the recycling of cellular components. In the early endosomal pathway the recycling of endocytosed receptors depends on the $\mathrm{pH}$, such as the separation of transferrin 


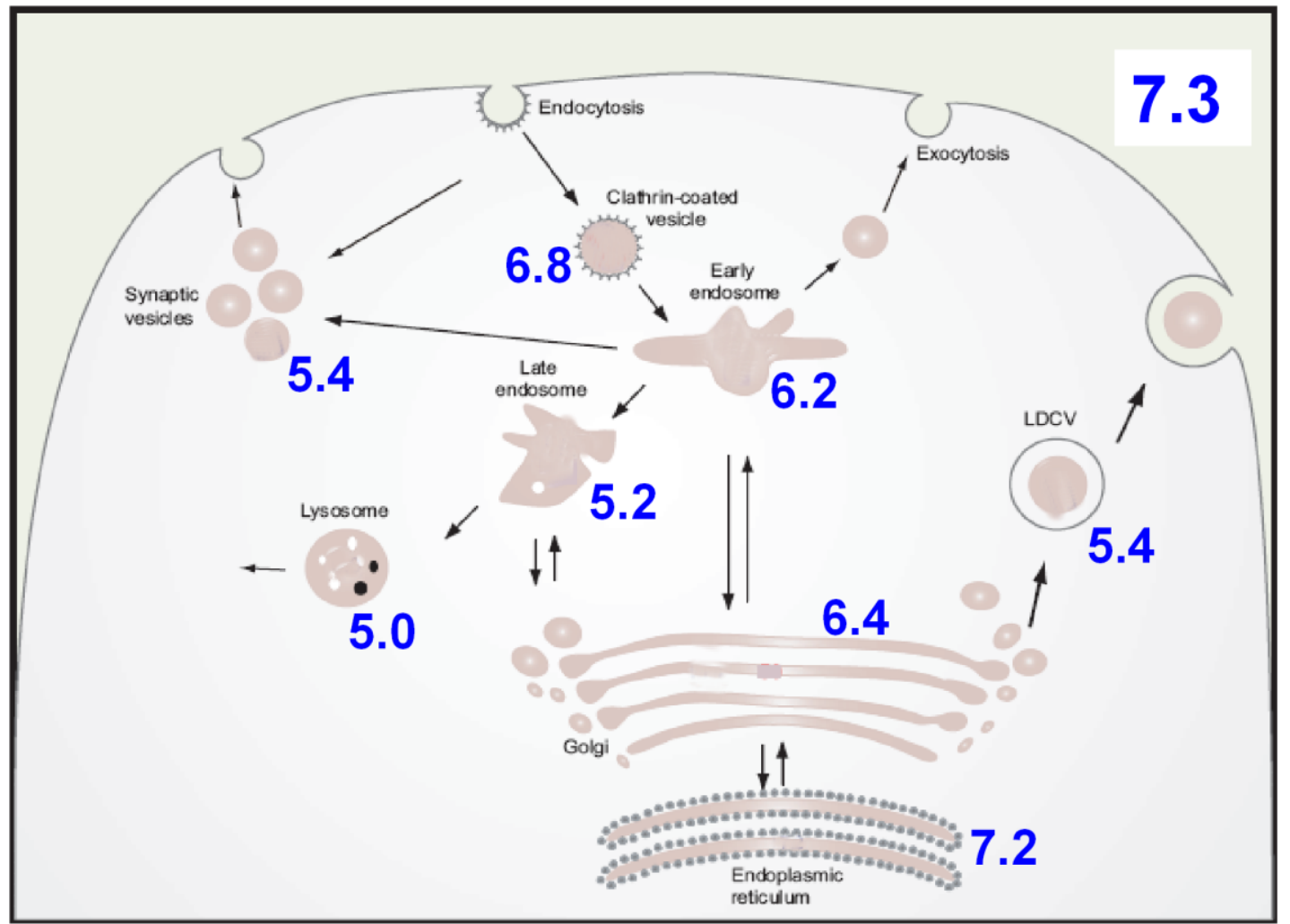

Figure 1.6

Acidification of intracellular compartments

Schematic representation showing the typical $\mathrm{pH}$ values of membraneous organelles in an animal cell. Except for the endoplasmatic reticulum the endomembrane system is more acidic than the cytosol ( pH 7.4). The most acidic compartments are the lysosomes with $\mathrm{pH}$ values of $\sim 5$. Slightly modified from Faundez and Hartzell, 2004.

from the transferrin receptor (Maxfield and McGraw, 2004), suggesting that the regulation of the $\mathrm{pH}$ is involved in trafficking events. For instance the trafficking of endocytosed membranes is disturbed in the absence of the chloride transporter $\mathrm{ClC}-5$ and has been interpreted as a result of impaired acidification (Piwon et al., 2000). Furthermore, the low $\mathrm{pH}$ is also necessary for transport processes across the membranes where protons are exchanged with the cytosolic substrates that are being imported such as acetylcholine or monoamines (see 1.3.7). Sialin (also a member of the Slc17 family like VGLUTs) for instance, a translocator of sialic acid, cotransports this acidic carbohydrate out of lysosomal compartments together with protons and thus uses a $\mathrm{pH}$ gradient for the efflux (Morin et al., 2004). Defects in the acidifcation of lysosomal compartments, among other reasons, can lead to deposits of cellular debris, a pathological condition that affects the entire organism (lysosomal storage diseases) (Ruivo et al., 2009). 
As mentioned earlier, the V-ATPase is an electrogenic pump, meaning it does not bear any conductances for counterion efflux or pairion influx. In the absence of such pathways the lipid bilayer provides a high resistance barrier and eventually translocated protons immediately build up a luminal positive membrane potential $(\Delta \psi)$. This potential will then bring the rotary pumping of the V-ATPase to halt. Therefore it is clear that paths for ions must provide an electrical shunt for the acidification of membraneous organelles (Grabe and Oster, 2001). The major factors that define the setpoint for the two components of $\Delta \mu_{\mathrm{H}+}$ are illustrated in figure1.7. The buffering capacity of the interior of vesicles provided by charges on luminal protein domains, e.g. by charged carbohydrates, the transport solutes themselves or endocytosed buffers such as phosphate or bicarbonate, is generally difficult to estimate. It can only be experimentally determined, which is very demanding in the case of SVs.

Importantly, isolated intracellular organelles like SVs acidify only in the presence of small anions like $\mathrm{Cl}^{-}$(Xie et al., 1989; Xie et al., 1983). This indicates that $\mathrm{Cl}^{-}$pathways provide a shunting current for the electrogenic V-ATPase and, thereby lead to an increase of $\Delta \mathrm{pH}$ at the expense of $\Delta \Psi$ by net accumulation of $\mathrm{HCl}$. It should be noted though, that isolated organelles probably do not reveal all of the conductivities that are present in the bilayer. For instance it could be that the vesicles acidify due to the presence of a cation channel

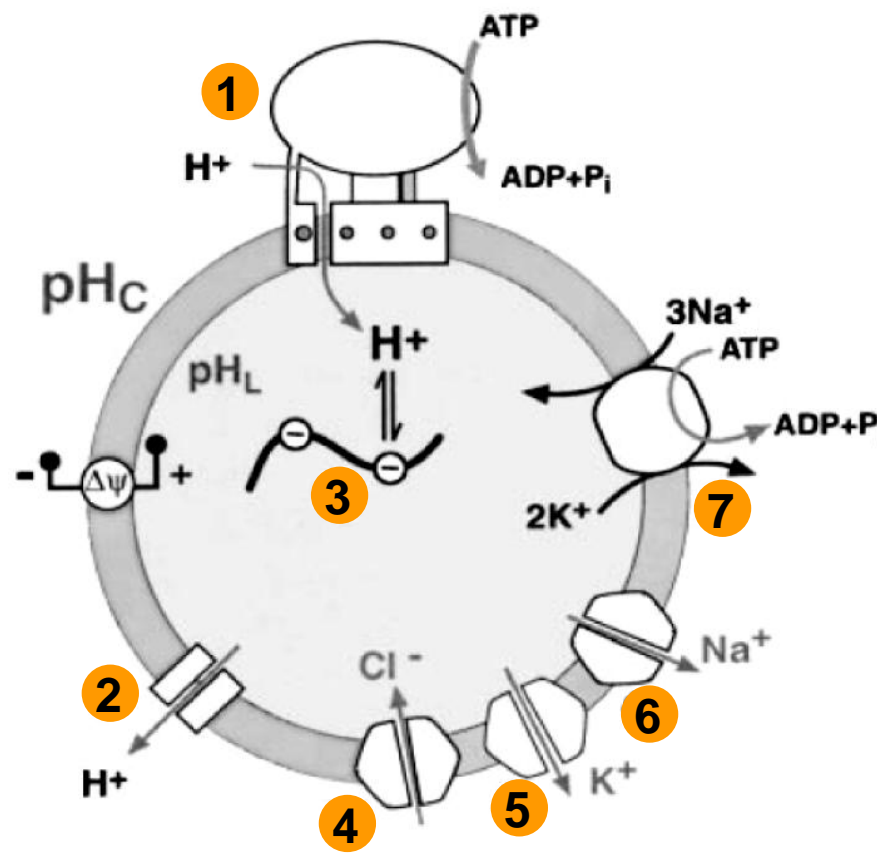

\section{Figure 1.7}

Determinants of vesicle acidification

The major determinants that define the contribution of the transmembrane potential $(\Delta \psi)$ and the $\mathrm{pH}$ gradient $(\Delta \mathrm{pH})$ to the proton electrochemical gradient $\left(\Delta \mu_{H_{+}}\right)$in a secretory vesicle.

The V-ATPase (1) pumps protons into the lumen. The resulting $\mathrm{pH}$ in the lumen is then defined by proton leaks (2), the buffering matrix (3) and shunting currents from channels that enable pair ion movement (4) or efflux of counter ions $(\mathbf{5 , 6})$. The $\mathrm{Na}^{+}-\mathrm{K}^{+-}$ATPase $(\mathbf{7})$ present to some extent also in some endosomal membranes influences also $\Delta \mu_{H_{+}}$. Slightly modified from Grabe et al.,2001. 
(counterion movement). These cations could have been endocytosed upon vesicle formation at the plasma membrane. If isolated vesicles lost these cations during the reacidifcation it will not be possible to reveal the presence of cation conductances in vitro. Nevertheless, some key experiments elegantly proved that SVs contain a conductance for anions while none for cations could be shown (Xie et al., 1989). For this, clathrin-coated vesicles (CCVs) from bovine brain were solubilized, and their constituents were then chromatographically separated. When the fractions were later reconstituted it was evident that an anion-conductivity in SVs is present and thus must underlie the observed low $\mathrm{pH}$ of SV lumina. If this anion conductance is evenly distributed among the SV subtypes (glutamatergic, GABAergic and monoamineergic) and in all cases is due to the same protein, remained unclear. The molecular identity of this putative channel could not be revealed and the underlying $\mathrm{Cl}^{-}$shunt in $\mathrm{SVs}$ has remained elusive for a long time.

\subsubsection{ClC-3, a candidate for the chloride channel on synaptic vesicles}

Chloride channels belong to several structurally unrelated classes and may not show any architectural features of cation channels. The molecularly identified $\mathrm{Cl}^{-}$channels can be sorted into several groups: Ligand gated $\mathrm{GABA}_{\mathrm{A}}$ and glycine receptors, that are related to the pentameric acetylcholine receptor, cystic fibrosis transmembrane conductance regulator (CFTR) a large $\mathrm{ABC}$ transporter, the intacellular $\mathrm{Cl}^{-}$channels (CLIC), belonging to the gluthathione $S$-transferase (GST) proteins that are soluble but can integrate into a bilayer and induce a $\mathrm{Cl}^{-}$conductance, and members of the CLC gene family. This listing is certainly incomplete, as some $\mathrm{Cl}^{-}$channels have not been identified yet (for instance the swelling activated $\mathrm{Cl}^{-}$channels) (Jentsch, 1996). Recently, a novel anion channel, that is critical for the acidification of the Golgi apparatus has been found, belonging also to a new group (Maeda et al., 2008). Among the $\mathrm{Cl}^{-}$channels that are most prominent on endomembranes and well characterized are the CLC channels (Jentsch et al., 2005). The CLC gene family is comprised by 9 different members in mammals. They have been originally identified in the electric organ of Torpedo (ClC-0) (Jentsch et al., 1990; Miller, 1982). Some of the CLC members are present on the plasma membrane (ClC-0 (Torpedo), $\mathrm{ClC}-1, \mathrm{ClC}-2$ and $\mathrm{ClC}-\mathrm{Ka} / \mathrm{b}$ ), others in intracellular membranes (ClC-3, ClC-4, ClC-5, $\mathrm{ClC}-6$ and $\mathrm{ClC}-7)$. They are involved in a broad range of functions such as the stabilization 
of the membrane potential, cell volume regulation, and transepithelial transport. Among this, certain isoforms are believed to be essential for the acidifcation and trafficking of numerous organelles such as endosomes (ClC-5) (Piwon et al., 2000) and lysosomes (ClC7) (Graves et al., 2008) and also involved in bone resorption at the resorption lacuna of osteoclasts (ClC-7) (Kornak et al., 2001). For the loss of ClC-7 additional phenotypes have been described, such as lysosomal storage disease and neurodegenration (Kasper et al., 2005).

The ClCs 3, 4, and 5 show 80\% homology and are also quite similar in their electrophysiological characteristics (Friedrich et al., 1999; Matsuda et al., 2008; Meyer et al., 2007; Picollo and Pusch, 2005; Scheel et al., 2005). While the function of ClC-4 is not well understood, the deletion of ClC-5 showed a clear phenotype, since it causes Dent's disease, a pathological condition that manifests in proteinuria (Piwon et al., 2000). This has been addressed to impaired endocytosis, though not completely abolished. It was furthermore shown that endosomes from $\mathrm{Clcn}^{-/}$mice had a lower rate and extent of acidification suggesting a function in anion shunting to dissipate the voltage generated by the V-ATPase (Hara-Chikuma et al., 2005a).

The deletion of $\mathrm{ClC}-3$ did not clearly reveal its function although the phenotype was confirmed in three independent mouse models (Dickerson et al., 2002; Stobrawa et al., 2001; Yoshikawa et al., 2002). The deletion of the gene leads to severe neurodegeneration in wide areas of the brain, including the complete loss of the hippocampus and the degeneration of the retina. This neurodegeneration is starting at the age of approximately three weeks. Surprisingly, the mice are capable of survival without the hippocampus, albeit an increased lethality and some behavioral abnormalities like hyperactivity (Stobrawa et al., 2001; Yoshikawa et al., 2002). The mechanism that leads to neurodegeneration remains unclear, but is probably due to trafficking defects (Stobrawa et al., 2001), and involves a massive increase of microglia indicating inflammatory-like responses. Furthermore, the loss of $\mathrm{ClC}-3$ is associated with a moderate storage of the c subunit of the mitochondrial ATP-Synthase in lysosomes, which is typical for lipofuscinosis (Yoshikawa et al., 2002). Since ClC-3 is not restricted to the brain, other tissues are also affected, such as the duodenum that shows necrotic patches (Yoshikawa et al., 2002). ClC-3 appeared cleary enriched in highly purified rat SVs because it colocalized with SV markers. In addition, ClC-3, has been an attractive candidate for $\mathrm{Cl}^{-}$shunting on $\mathrm{SV}$ s based on the fact 
that the acidification of SVs derived from $\mathrm{Clcn}^{-/-}$mice is partially impaired (Stobrawa et al., 2001). However, the loss of ClC-3 does not cause major changes in neurotransmission and vesicular glutamate transport of isolated vesicles retains its biphasic dependence on $\mathrm{Cl}^{-}$ even in the absence of ClC-3 (Stobrawa et al., 2001). This is surprising, since the loss of a $\mathrm{Cl}^{-}$shunt would be expected to influence $\Delta \mathrm{pH}$ and consequently also glutamate loading as described in 1.3.6 and 1.3.7. In support of a function in anion shunting has been a study that indicated lower $\mathrm{Cl}^{-}$content and less acidifcation in endosomes from $\mathrm{Clcn}^{-/}$mice using fluorescent internalized dyes (Hara-Chikuma et al., 2005b). Despite the lack of an influence on the biphasic dependence of vesicular glutamate transport, $\mathrm{ClC}-3$ has been widely accepted as the $\mathrm{Cl}^{-}$channel on SVs.

Notably, one of the best characterized $\mathrm{ClCs}$ is a homologue from E. coli, $\mathrm{ClCec} 1$, which is essential in the immediate acid response (Iyer et al., 2002). ClCec1 was amenable to purification in reasonable quantities that helped to study $\mathrm{ClC}$ function by physiological and structural methods (Accardi et al., 2004). It represents the only $\mathrm{ClC}$ protein that has been crystallized to date but due to the high homology with eukaryotic ClCs the overall architecture of this protein family was revealed (Dutzler et al., 2002). The most surprising finding though was that $\mathrm{ClCec} 1$ is in fact a $\mathrm{Cl}^{-} / \mathrm{H}^{+}$exchanger and not a $\mathrm{Cl}^{-}$channel (Accardi and Miller, 2004). This finding had far reaching consequences for the proposed functions in the intracellular $\mathrm{ClC}$ channels 3-7. This is because $\mathrm{ClCec} 1$ contains a signature consisiting of only one amino acid that can abolish the $\mathrm{H}^{+}$dependence in the countertransport. Upon mutation of glutamate 148 to alanine (inferred from the high resolution structure), $\mathrm{ClCec} 1$ behaves like a $\mathrm{Cl}^{-}$channel, resembling more $\mathrm{ClC}-0$. Furthermore the pronounced outward rectification is removed by this mutation. Since E148 is well conserved among intracellular $\mathrm{ClCs}$ the presence of this conserved residue suggests functional differences between intracellular and plasma membrane ClCs. Mutation of the corresponding glutamate residue in $\mathrm{ClC}-5$ also leads to channel-like behaviour and abolishes the outward rectification (Meyer et al., 2007).

Recently, indications for a $\mathrm{Cl}^{-} / \mathrm{H}^{+}$exchange have been found for $\mathrm{ClC}-3$ (Matsuda et al., 2008). This has also been demonstrated for ClC-4/5 (Picollo and Pusch, 2005; Scheel et al., 2005) and ClC-7 (Graves et al., 2008). The $\mathrm{Cl}^{-} / \mathrm{H}^{+}$exchange mechanism does not exclude a role in acidification. But it nevertheless complicates the interplay with a proton pump and together with the pronounced outward rectification of intracellular $\mathrm{ClCs}$ (Friedrich et al., 
1999; Matsuda et al., 2008), the observed cotransport of $\mathrm{Cl}^{-}$and $\mathrm{H}^{+}$in isolated SVs cannot be well explained. In addition it appears not economical to exchange luminal protons with cytosolic $\mathrm{Cl}^{-}$to acidify the lumen of a vesicle. It was suggested that the key to the shunting function lies within the stoichiometry of the transport (Edwards, 2007) which has been shown to be $2 \mathrm{Cl}^{-} / 1 \mathrm{H}^{+}$(Accardi and Miller, 2004; Graves et al., 2008). This notion however does not take the outward rectification of the intracellular ClCs into account. The electrophysiogical characteristics of these $\mathrm{Cl}^{-}$translocators make a transport of $2 \mathrm{Cl}^{-}$ions out of the lumen in exchange with one proton most likely, since the membrane potential of a SV is presumably inside positive due to the pumping activity of the V-ATPase. This transport would however be immediately arrested because it is electrogenic, further increasing the membrane potential $\left(+1 \mathrm{H}^{+}\right)$. An interesting idea is therefore that $\mathrm{ClCs}$ might reset the membrane potential upon efflux of positively charged solutes. For this, luminal $\mathrm{Cl}^{-}$ions that should be abundantly present in endosomal structures after endocytosis could be used. Compatible with this hypothesis is the finding that $\mathrm{ClCs}$ seem to play a role in trafficking of endosomes, which involves also fusion reactions. These fusion reactions require $\mathrm{Ca}^{2+}$ which originates from luminal stores of the endosomes themselves (Bayer et al., 2003; Holroyd et al., 1999). It is therefore an attractive idea that ClCs play a role in such events as recently proposed (Picollo and Pusch, 2005), however, currently there is no supporting experimental evidence available.

\subsection{Synaptic vesicle acidifcation and glutamate uptake: Are glutamate loading and anion shunting separate units ?}

Since the ratio of $\Delta \Psi$ and $\Delta \mathrm{pH}$ profoundly influences the uptake of glutamate, and also other transmitters, the molecular signature of the $\mathrm{Cl}^{-}$channel is crucial in the understanding of the loading process and its regulation. To date $\mathrm{ClC}-3$ is the most favored candidate for anion shunting despite some arguments speaking against such a function (see $1.4 .1)$

Interestingly, the loss of ClC-3 is accompanied by a decrease of the VGLUT1 content in SV fractions and of glutamate uptake into SVs (Stobrawa et al., 2001), presumably due to neurodegeneration including the complete loss of hippocampus. Importantly, the hippocampus is an area with strong VGLUT1 expression (Fremeau et al., 2001; Yoshida et 
al., 2008). Analysis of brains from $\mathrm{Clcn} 3^{-/}$mice before the onset of neurodegeneration are currently missing, thus making it difficult to rule out secondary effects that could arise from this neurodegenerative phenotype (but see this work).

VGLUT1, in turn, has been shown to induce a $\mathrm{Cl}^{-}$conductance when heterologously expressed, which can be inhibited by glutamate (Bellocchio et al., 2000), a strong indication for a $\mathrm{Cl}^{-}$conductance in the transporter itself. These observations cast doubts if the observed partial reduction in $\mathrm{Cl}^{-}$-dependent acidification in $\mathrm{SVs}$ from $\mathrm{Clcn} 3^{-/-}$brains is caused by the loss of $\mathrm{ClC}-3$, but suggest that it is an indirect effect linked to the reduction of VGLUT1. VGLUT-positive SVs account for the majority of isolated SVs. Therefore any role of VGLUT1 in anion shunting should be rather dominant in measurements of vesicle acidification. In view of the biphasic dependence of glutamate transport on extravesicular $\mathrm{Cl}^{-}$and the indications for direct binding of $\mathrm{Cl}^{-}$(Hartinger and Jahn, 1993), there is a clear need to elucidate the contribution of VGLUT1 in this context and to unmask the molecular identity of the $\mathrm{Cl}^{-}$translocator on SVs in order to gain a correct understanding of glutamate loading.

\subsection{Aim of this work}

The molecular identity of the $\mathrm{Cl}^{-}$channel on SVs has been a longstanding question. It is believed that this $\mathrm{Cl}^{-}$channel plays a vital role for the loading of neurotransmitters by modulating the proton electrochemical gradient $\Delta \mu_{\mathrm{H}+}$ (see 1.4). For instance, it is known since decades, that the uptake of glutamate, the major excitatory transmitter in the vertebrate brain, into isolated SVs is exhibiting an unusual biphasic response towards $\mathrm{Cl}^{-}$in the assay medium (see 1.3.6).

ClC-3, a member of the CLC gene family, is a $\mathrm{Cl}^{-}$translocator that localizes to intracellular membranes and appeared to be enriched in SVs. Since the deletion of the ClC-3 gene lead to a reduction in the rate of vesicle acidification it was suggested that $\mathrm{ClC}-3$ is the $\mathrm{Cl}^{-}$ channel on SVs. However, some doubts remained. First, ClC-3 leads to severe neurodegeneration, naturally in brain areas where it is normally expressed. Since these brain areas are absent in any subsequent experiment, it is difficult to draw a conclusion with certainty about the function of $\mathrm{ClC}-3$ in exactly these brain areals. Furthermore, 
vesicles isolated from $\mathrm{Clcn} 3^{-/}$mice, after severe neurodegeneration already affected the brain, showed a decrease in the level of the major vesicluar glutamate transporter, VGLUT1, indicating that the vesicle composition might be affected. Second, the typical biphasic response of vesicular glutamate transport towards the extravesicular $\mathrm{Cl}^{-}$ concentration has been attributed to the presence of a $\mathrm{Cl}^{-}$channel on SVs. This biphasic response is however still present in $\mathrm{Clcn3}^{-/-}$mice SV isolates (Stobrawa et al., 2001).

Recently, indications for a $\mathrm{Cl}^{-}$conductance in VGLUT1, the transporter responsible for glutamate translocation on SVs, were found by heterologously expressing the gene on secretory vesicles (Bellocchio et al., 2000). A direct interaction of VGLUT1 with $\mathrm{Cl}^{-}$has already been proposed before, but it was not interpreted as the result of a conductance. Instead the transporter was believed to be regulated by $\mathrm{Cl}^{-}$due to an allosteric binding site that would modulate the glutamate translocation (Hartinger and Jahn, 1993; Juge et al., 2006). Further investigations to analyze this phenomenon in depth have been hampered by the difficulties in studying vesicular transporters in general. Since the transporters reside in intracellular compartments they are less accessible for physiological studies. Additionally the use of ionophores is relatively restricted because only the extravesicular side of the vesicles can be defined in its ionic composition. In the case of VGLUTs, investigations are further hindered by its low intrinsic affinity for glutamate, resulting in relatively low signal to noise ratios in experiments.

The recently established Vglut $^{-1}$ mouse strain makes it possible to reevaluate the $\mathrm{Cl}^{-}$ conductance of SVs in the light of the above mentioned findings. It is now feasible to directly compare SVs from $\mathrm{Clcn}^{-{ }^{--}}$mice with SVs from mice lacking VGLUT1. One major aim of this work was therefore to clarify the role of these two candidates by comparing the extent of acidifaction in isolated SVs from either mutant mice. An indication for a $\mathrm{Cl}^{-}$conductance in the Vglutl $^{-/}$strain would then justify a deeper investigation of the function of this conductance. It was therefore hypothesized that this conductance itself is regulating the uptake of glutamate into $\mathrm{SVs}$ and that $\mathrm{Cl}^{-}$is directly involved in the translocation process in VGLUT1, probably as a substrate.

A detailed characterization of the glutamate translocation should be preferentially done in a tightly controlled environment free of contaminants, and simple enough for the interpretation of experimental results. A membrane preparation that is essentially free of possibly interfering conductances and that can be reliably energized with a proton pump 
would serve as the ideal basis to study the VGLUT1 characteristics. To accomplish this, it was attempted to purify the transporter from a recombinant source and reconstitute it into artificial liposomes, energized with a purified proton pump. Although it is problematic to purify a polytopic eukaryotic membrane protein with the size of VGLUT1 in reasonable amounts, it was possible to achieve this by heterologous expression in a HEK293 cell line. The second major aim of this work was therefore to verify the $\mathrm{Cl}^{-}$conductance for VGLUT1, not only by analysis of SVs from Vglut $^{-/}$mice, but also in an independent manner with purified components. Furthermore, the formation of artificial vesicles would allow for a control over the intravesicular and extravesicular ion composition and the usage of ionophores, thus making a more detailed characterization of the glutamate transport possible, in particular to clarify the role of $\mathrm{Cl}^{-}$ions in the translocation process. The vesicular transport of glutamate is currently believed to be depend largely on $\Delta \psi$, however the proposed transport mode is inconsistent with some theoretical limitations and experimental observations for the loading of SVs. For instance, questions regarding the pairion of glutamate are not fully compatible with biophysical restrictions. This includes a much lower $\mathrm{pH}$ of the vesicle interior than actually measured if it was $\mathrm{H}^{+}$and an osmotic pressure in the vesicle that would likely exceed the upper limit if glutamate was stored only as a free acid. Furthermore the currently described uptake kinetics from in vitro experiments with isolated SVs are probably not fast enough to explain the reloading of glutamate at the synaptic terminal. It could therefore be that important parameters, i.e. regarding the internal ion composition of SVs just after endocytosis, have not been taken fully into account, in order to reach towards a biophysically coherent understanding of glutamate loading. The answer of such questions should be greatly facilitated by the usage of purified transport components in artificial vesicles, which makes a control over previously inaccessible parameters possible. 


\section{MATERials AND MethodS}

\subsection{Animal keeping and crossing}

The mice used in this study were kept in a 12 hour light-dark cycle with food and water $a d$ libitum. Mouse keeping and experiments followed protocols approved by the Institutional Animal Care and Use Committee of Tokyo Medical and Dental University.

The Vglut1-mutant strain (Wojcik et al., 2004) was established by donation of 5 heterozygous male mice from Drs. Sonja M. Wojcik and Nils Brose (Dep. of Molecular Neurobiology, MPI for Experimental Medicine, Göttingen). The mice were then crossed with B6 mice.

The Clcn3 mutant strain (Yoshikawa et al., 2002) was donated by Drs. S. Sasaki and and S. Uchida (Dep. of Nephrology, Tokyo Medical and Dental University). Like for Vglut1 mutants, the mice were crossed with B6 mice.

In order to get a sufficiently high number of synchronized KO-offspring for both VGlut1 and Clcn3 mutant mice, it was necessary to cross a relatively high number of heterozygous animals. Typically a 2-4 female mice per cage were mated with one male mouse for 4 days (within a mouse cycle to ensure synchronized birth). In a different approach in vitro fertilization (IVF) was used to achieve simultaneous birth of up to $25 \mathrm{KO}$-animals. This service was offered by an in-house facility but could only be used twice for the first generations of Vglut1 mutant mice.

Vglut $^{-/-}$mice are generally weak from birth on (Fremeau et al., 2004; Wojcik et al., 2004), however their condition worsens considerably after 2-3 weeks of age, in line with an increased expression of the VGLUT1 protein in this developmental stage (Boulland et al., 2004; Nakamura et al., 2005). In order to investigate samples from knockout animals with an age of two months, special care was necessary to keep the animals alive. It was necessary to serve wet soft food, water gel, occasionally warm the cage from outside with an IR-lamp and keep the surroundings as hygenic as possible. Since the wet food had to be supplied on the ground and the knockout mice are blind the cage easily became very dirty. Apparently the knockout mutants had also sensory deficits and occasionally injured 
themselves (especially the tail was often wounded), or were not able to "take care" of themselves (e.g. they were often wet because they walked through the food and water supplies). This led also to infections. Although no detailed statistics were made, about $30 \%$ of the knockout mice born could survive up two 2-3 months with special care taken. A key was the right time point to separate the knockout individuals, first from their littermates to careful mothers and then later just before the end of the weaning period from the mothers to aquire essential abilities for survival before reaching 2-3 weeks of age.

The $C l c n 3^{--}$animals did not need special care like Vglutl mutants, however they were also blind and suffered also from a higher mortality after 3 weeks of age similar to previously described observations (Stobrawa et al., 2001).

\subsection{Isolation of synaptic vesicles from mouse brains}

After the genotyped mice reached the desired age, equal numbers of mice per genotype were used for the preparation of synaptic vesicles. The individuals did not differ by more than 3 days in their age, except for some preparations with mice over the age of 2 months.

A subcellular fraction of whole brain homogenates that is enriched in synaptic vesicles (LP2, light pellet 2) was chosen to be used for investigating the acidification of synaptic vesicle lumina. Despite the fact that synaptic vesicles, purified by immunoisolation or chromatographic methods would be preferable, the LP2 fraction (containing approx. $\sim 1 / 5^{\text {th }}$ of synaptic vesicle protein than the purest synaptic vesicles achievable) offers still a good compromise between the number of mice needed for a preparation and the specificity of the signal in later applications.

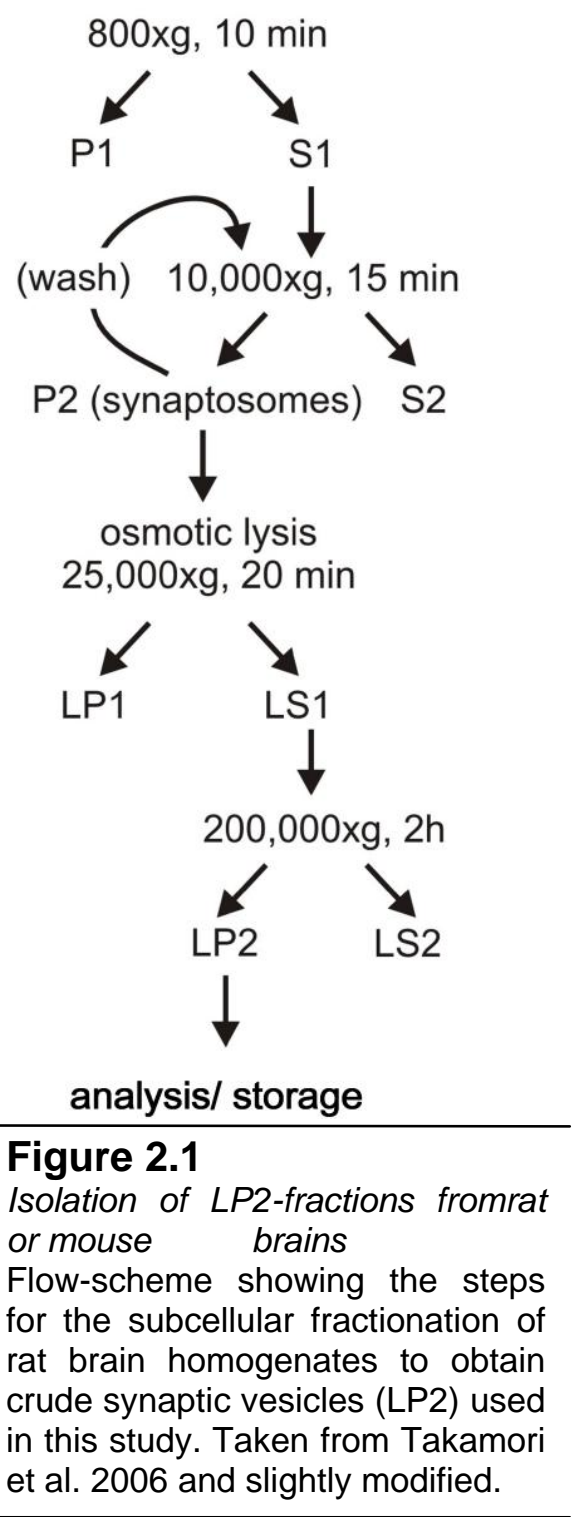


LP2 was prepared based on a published protocol (Hell et al., 1994). A flow-scheme of the preparation can be seen in figure 2.1. The samples were resuspended in $320 \mathrm{mM}$ sucrose and adjusted to a protein concentration of $2.5 \mathrm{mg} / \mathrm{ml}$. Aliquots were frozen in liquid nitrogen and stored at $-80^{\circ} \mathrm{C}$.

\subsection{Heterologous expression and purification of proton pumps}

\subsubsection{Purification of $E$. coli wild type ATP-Synthase $\left(\mathbf{F}_{0} \mathbf{F}_{1}\right)$}

Wild type E. coli ATP-Synthase $\left(\mathrm{F}_{0} \mathrm{~F}_{1}\right)$ was purified on the base of a slightly modified protocol published previously (Moriyama et al., 1991). The DK8-strain carrying pBWU13 (DK8/pBWU13) was kindly donated by Dr. Y. Moriyama (Dep. of Pharmacology, University of Okayama).

For the expression of the $\mathrm{F}_{0} \mathrm{~F}_{1}$-complex a single colony from a Amp-LB plate was picked from a freshly streaked out glycerol-stock (stored at $-80^{\circ} \mathrm{C}$ ) and cultivated for $\sim 8$ hours in $\sim 5 \mathrm{ml}$ liquid LB (Luria-Bertani medium) (Sambrook et al., 2001), containing $100 \mu \mathrm{g}$ ampicillin. One liter of minimal medium (Moriyama et al., 1991) containing $0.5 \%$ glycerol (the sole carbon source), but no ampicillin, was then inoculated with the preculture (1:500) and grown at $37^{\circ} \mathrm{C}$ until an $\mathrm{OD}_{600}$ of $\sim 1-1.5$ was reached ( 24 hours).

The cells were then harvested by centrifugation and resuspended in $10 \mathrm{ml}$ of $0.5 \mathrm{M}$ sucrose, $50 \mathrm{mM}$ Tris-Cl, $\mathrm{pH}$ 8.0. $50 \mathrm{mg}$ lysozyme, $1 \mu \mathrm{g} / \mathrm{ml}$ Leupeptin, $1 \mu \mathrm{g} / \mathrm{ml}$ Pepstatin and $1 \mathrm{mg} / \mathrm{ml} \mathrm{DNase} \mathrm{I} \mathrm{were} \mathrm{added} \mathrm{and} \mathrm{the} \mathrm{suspension} \mathrm{was} \mathrm{incubated} \mathrm{for} 1.5$ hours at $30^{\circ} \mathrm{C}$. Then EDTA ( $\mathrm{pH} 8.0$ ) was added to $5 \mathrm{mM}$ and the suspension was incubated for additional $30 \mathrm{~min}$. Then the suspension was diluted 10 fold with water containing $1 \mu \mathrm{g} / \mathrm{ml}$ Leupeptin, $1 \mu \mathrm{g} / \mathrm{ml}$ Pepstatin, $1 \mathrm{mM}$ PMSF and $5 \mathrm{mM}$ dithiothreitol (DTT). Afterwards the suspension was tip-sonicated (Branson S250A) for $5 \times 30$ s at an output of 3-4 and 30\% duty cycle. Then glycerol was added to $10 \%$ and the suspension was centrifuged at $4500 \mathrm{rpm}$ at $4^{\circ} \mathrm{C}$ in a Beckmann JA-20 rotor. The turbid supernatant was collected and spun at 50,000 rpm in a Beckmann Ti 70 rotor for $45 \mathrm{~min}$ at $4^{\circ} \mathrm{C}$. The brown opaque pellet was resuspended in 1 $\mathrm{ml}$ of $10 \mathrm{mM}$ Tris-Cl $\mathrm{pH} 8,5 \mathrm{mM}$ DTT, $2 \mathrm{mM} \mathrm{MgCl}_{2}, 10 \%$ glycerol and proteinase 
inhibitors $(1 \mu \mathrm{g} / \mathrm{ml}$ Leupeptin, $1 \mu \mathrm{g} / \mathrm{ml}$ Pepstatin). The membranes were snap frozen in liquid $\mathrm{N}_{2}$ and stored at $-80^{\circ} \mathrm{C}$.

For the purification of $\mathrm{F}_{0} \mathrm{~F}_{1}$ the membranes were thawed and washed in in $10 \mathrm{mM}$ Tris-Cl $\mathrm{pH} 8,5 \mathrm{mM}$ DTT, $2 \mathrm{mM} \mathrm{MgCl}_{2}$, proteinase inhibitors ( $1 \mu \mathrm{g} / \mathrm{ml}$ Leupeptin, $1 \mu \mathrm{g} / \mathrm{ml}$ Pepstatin) and $0.8 \%$ n- $\beta$-octylglucoside (OG, final concentration from $20 \%$ OG-stock, added just prior to the next step) in a final volume of $6 \mathrm{ml}$. The membranes were pelleted immediately in a Beckman 100.4-rotor with $70,000 \mathrm{rpm}$ for $30 \mathrm{~min}$ at $4^{\circ} \mathrm{C}$. Then the membranes were resuspended in $10 \mathrm{mM}$ Tris- $\mathrm{Cl}, 2 \mathrm{mM} \mathrm{MgCl}_{2}, 5 \mathrm{mM}$ DTT, $2 \%$ OG and proteinase inhibitors as above with a small potter homogenizer for incubation on ice for 30 minutes. The lysate was spun again at $70,000 \mathrm{rpm}$ for $30 \mathrm{~min}$ at $4^{\circ} \mathrm{C}$. Afterwards the supernatant was subjected to a glycerol density gradient (in $10 \mathrm{mM}$ Tris- $\mathrm{Cl} \mathrm{pH} 8,5 \mathrm{mM}$ DTT, 2 mM MgCl $25-30 \%$ glycerol,1 \% OG) in a Beckmann SW55 rotor with (52,000 rpm (330,000 $\left.\mathrm{g} R_{\max }\right)$ for $5 \mathrm{hrs}$ at $4^{\circ} \mathrm{C}$. For this, maximal $0.5 \mathrm{ml}$ of supernatant was placed on top of $4.5 \mathrm{ml}$ step-gradient. The gradient was prepared in a cold room with glycerol contents of 30, 25, 20, 15, 10 and 5\% glycerol. Fractions were collected from bottom to top with a peristaltic pump and a glass capillary in sizes of $\sim 300 \mu 1$. After analysis with SDSPAGE the fractions enriched in $\mathrm{F}_{0} \mathrm{~F}_{1}$ holoenzyme were pooled, snap-frozen in liquid $\mathrm{N}_{2}$ and stored at $-80^{\circ} \mathrm{C}$.

\subsubsection{Purification of Bacillus sp. PS3 thermophilc ATP-Synthase $\left(\mathbf{T F}_{0} \mathbf{F}_{1}\right)$}

ATP synthase holoenzyme $\left(\mathrm{TF}_{0} \mathrm{~F}_{1}\right)$ from the thermophilic Bacillus sp. PS3 was constitutively expressed in the E.coli strain DK8 (native unc operon deleted) from the plasmid pTR19ASDS (Suzuki et al., 2002). The $\beta$-subunit, which is present in three copies per complex is N-terminally hexa-His-tagged allowing affinity purification. The cells were grown to an OD600 of 1.5-2 in the presence of 100 $\mu \mathrm{g}$ ampicillin per $\mathrm{ml}$ in $2 \mathrm{~L}$ TB (terrific broth) (Sambrook et al., 2001) and harvested by centrifugation. The pellets were resuspended in $50 \mathrm{mM}$ Tris- $\mathrm{Cl}(\mathrm{pH} 8.0), 0.5 \mathrm{mM}$ EDTA and $1 \mathrm{mg} / \mathrm{ml}$ lysozyme and incubated at $37{ }^{\circ} \mathrm{C}$ under gentle shaking for 1 hour. $\mathrm{MgCl}_{2}$ was then added to a final concentration of $5 \mathrm{mM}$ and the suspension was sonicated 2 min on ice. Subsequently, DNaseI, $\mathrm{Na}_{2} \mathrm{SO}_{4}$ and sodium cholate were added to reach final concentrations of $1 \mu \mathrm{g} / \mathrm{ml}$, $250 \mathrm{mM}$ and $0.7 \%$ (wt/vol), respectively. The suspension was stirred for $20 \mathrm{~min}$ at $25^{\circ} \mathrm{C}$ 
and the spun at $20,000 \mathrm{~g}$ at $4^{\circ} \mathrm{C}$. After this washing step, the pellet was resuspended in $100 \mathrm{mM} \mathrm{KCl}, 20 \mathrm{mM}$ Imidazole, $5 \mathrm{mM} \mathrm{MgCl} 2$ and $1 \%$ n-dodecyl- $\beta$-D-maltopyranoside (DDM) (wt/vol), pH 7.6 stirred for $45 \mathrm{~min}$ at $25{ }^{\circ} \mathrm{C}$ and then subjected to centrifugation with $20,000 \mathrm{~g}$ at $4^{\circ} \mathrm{C}$. The resulting supernatant was batch incubated with $\sim 2-3 \mathrm{ml}$ Talon beads (Clontech) to capture $\mathrm{TF}_{0} \mathrm{~F}_{1}$ via its tagged $\beta$-subunit. The beads were washed with 10 column volumes of $100 \mathrm{mM} \mathrm{KCl}, 20 \mathrm{mM}$ Imidiazole, $5 \mathrm{mM} \mathrm{MgCl} 2$ and $0.08 \% \mathrm{DDM}$ at $\mathrm{pH}$ 7.6. Elution of the complex was achieved with $250 \mathrm{mM}$ Imidazole, $50 \mathrm{mM} \mathrm{KCl}, 5 \mathrm{mM}$ $\mathrm{MgCl}_{2}, 0.05 \%$ DDM. The complex could be used already with the purity achieved, but was purified further except for some initial test experiments. For this the eluate from the IMAC (immobilized metal affinity chromatography) step was dialyzed against $20 \mathrm{mM}$ $\mathrm{NaCl}, 20 \mathrm{mM}$ Hepes- $\mathrm{Na}$ and $5 \mathrm{mM} \mathrm{MgCl}_{2}, \mathrm{pH} 7.5$ for 3 hours at $25^{\circ} \mathrm{C}$ or overnight at $4^{\circ} \mathrm{C}$. After ultrafiltration through a $0.22 \mu \mathrm{m}$ membrane, the sample was injected onto a MonoQ anion exchange column (GE, 5/50) operated on an ÄKTA-System (ÄKTA explorer, GE) at $4^{\circ} \mathrm{C}$ and eluted with a step gradient (4 column volumes (CV) 0-18\% Buffer B, 3 CV 18-60 $\%$ Buffer B, 11 CV 60-100 \% Buffer B) with Buffers A (20 mM NaCl, 20mM Hepes-Na, 5 $\mathrm{mM} \mathrm{MgCl} 2,0.05 \%$ DDM, pH 7.5) and B (1 M NaCl, 20mM Hepes-Na, $5 \mathrm{mM} \mathrm{MgCl}$, $0.05 \%$ DDM, pH 7.5). The eluent flow was $1 \mathrm{ml} / \mathrm{min}$ for $18 \mathrm{CV}$. Fractions were collected along the gradient between $18 \%$ and $65 \%$ B in $300 \mu$ steps. This concentrated the sample about 10 -fold. The peak fractions were pooled and subjected to a final polishing step (size exclusion chromatography, SEC) to remove eventually occuring $\mathrm{F}_{0}$ or $\mathrm{F}_{1}$ domains from the $\mathrm{F}_{0} \mathrm{~F}_{1}$ complex. About 600-800 $\mu \mathrm{l}$ were injected onto a Superdex $200 \mathrm{HC}$ 10/30 SECcolumn (GE) and eluted with $100 \mathrm{mM} \mathrm{KCl}, 10 \mathrm{mM}$ Hepes-K, $5 \mathrm{mM} \mathrm{MgCl}_{2}, 0.05 \%$ DDM, $\mathrm{pH} 7.4$ at a flow of $0.3 \mathrm{ml} / \mathrm{min}$ for $1.5 \mathrm{CV}$ at $4^{\circ} \mathrm{C}$. Fractions of $0.5 \mathrm{ml}$ were collected. After analysis on SDS-PAGE the $\mathrm{TF}_{0} \mathrm{~F}_{1}$ containing fractions were pooled, protein concentrations were measured and the complex was stored at $4{ }^{\circ} \mathrm{C}$. Yields up to $10 \mathrm{mg}$ purified $\mathrm{TF}_{0} \mathrm{~F}_{1}$ from $2 \mathrm{~L}$ culture were reached. 


\subsection{Heterologous expression and purification of VGLUTs in tsA201 cells}

\subsubsection{Cell culture and transfection}

The tsA201 cells (HEK293 cells stably transformed with the large T-antigen of SV40) were cultivated in DMEM (Dulbecco's modified Eagle's medium, Sigma) with $4.5 \mathrm{~g} \mathrm{l}^{-1}$ glucose supplemented with $2 \mathrm{mM}$ L-glutamine, $1 \mathrm{mM}$ sodium pyruvate, $50 \mathrm{U} \mathrm{ml}^{-1}$ penicillin, $50 \mu \mathrm{g} \mathrm{ml}^{-1}$ streptomycin and $10 \%$ fetal bovine serum (FBS, from varying sources) under a $5 \% \mathrm{CO}_{2}$ atmosphere at $37^{\circ} \mathrm{C}$. For expression purposes on a large scale, typically $10010 \mathrm{~cm}$ dishes were transfected with $2 \mathrm{mg}$ of plasmid-DNA with the calcium phosphate method (Chen and Okayama, 1987) at 60-70\% confluency. For a single dish 20 $\mu \mathrm{g}$ DNA and $125 \mathrm{mM} \mathrm{CaCl}_{2}$ (from a $2.5 \mathrm{M}$ stock) final were adjusted to $500 \mu 1$ with water. Then $500 \mu 1$ 2x BBS with a pH of 6.95 (BES-buffered saline; $50 \mathrm{mM}$ BES, 280mM NaCl, $1.5 \mathrm{mM} \mathrm{Na}_{2} \mathrm{HPO}_{4}$ ) was added. A very slight turbidity of the mixture became apparent after some seconds. The transfection mix $(1 \mathrm{ml})$ was added to the cells (10ml medium, exchanged about an hour before transfection) dropwise, and the cells were transferred to an incubator set to $2.8 \% \mathrm{CO}_{2}$. DNA-Ca $3\left(\mathrm{PO}_{4}\right)_{2}$ particles could be easily observed under the microscope leading to a milky appearance of the cell medium in the dish. For large scale transfection this protocol was scaled up. On the next day the $\mathrm{CO}_{2}$ level was set again to $5 \%$, the medium was changed and expression was continued for another 24 hours. Strong expression of VGLUT1 protein correlated with rounding up of cells. This could be easily visualized with GFP-tagged VGLUT1 (Fig. 3.12).

In order to reach a suitable density for the expression of VGLUT1, the last seeding was performed not more than one day prior to transfection in dilutions of 1:6-1:4 depending on the density of the source culture. Typically 15-25 very densely grown dishes were taken to reach the desired density resulting in appropriate spacing between the cells that would make about one more division per cell possible. 


\subsubsection{Purification of recombinant tagged VGLUT1}

Recombinant rVGLUT1 (see 2.10.3 for details on the DNA-construct) was purified in a single step making use of the nanomolar affinity of the SBP-tag (Keefe et al., 2001) for streptavidin and the gentle elution with (+)-biotin.

Transfected tsA201 cells (see 2.4.1) could be easily harvested by centrifugation and for the amount of 100 dishes the pellets were resuspended in $80 \mathrm{ml}$ of ice-cold Buffer A (300mM $\mathrm{KCl}, 40 \mathrm{mM}$ Tris-Cl and $2 \mathrm{mM}$ EDTA at $\mathrm{pH}$ 7.5). The cells were then lysed after the addition of $5 \mathrm{mM} \beta$-mercaptoethanol, $1 \mathrm{mM}$ phenylmethylsulfonylfluoride (PMSF) and solid DDM (2\% wt/vol) at $4{ }^{\circ} \mathrm{C}$ for $30 \mathrm{~min}$ under stirring. A huge clump of genomic DNA that forms upon lysis was sheared with scissors. Insoluble matter was pelleted by centrifugation $\left(300,000 \mathrm{~g}, 4^{\circ} \mathrm{C}\right)$ and the supernatant was cleared by an additional ultrafiltration to remove an insoluble buoyant low density fraction (this are lipids, most likely long-chain sphingolipids) and incubated in batch with $\sim 1 \mathrm{ml}$ streptavidin beads (UltraLink, Pierce) for 3 hours at $4{ }^{\circ} \mathrm{C}$ under rotation. The beads were then washed with 20 CVs of Buffer A (supplemented with $5 \mathrm{mM} \beta$-mercaptoethanol and 0.08\% DDM). Elution was achieved by incubation of the beads with $1 \mathrm{ml}$ of elution buffer $(100 \mathrm{mM} \mathrm{KCl}, 13 \mathrm{mM}$ Tris-Cl, $0.6 \mathrm{mM}$ EDTA, 2mM (+)-biotin, $5 \mathrm{mM} \beta$-mercaptoethanol and 0.04\% DDM, $\mathrm{pH} 7.5$ ) for $10 \mathrm{~min}$ on ice. The beads were spun at 5000g for $1 \mathrm{~min}$ and the supernatant was carefully taken off. This was repeated 5 times with fresh elution buffer. The resulting supernatants were pooled and concentrated in Amicon spin concentrators (50kDa cutoff, Millipore) in a few minutes on a swing-out rotor for $50 \mathrm{ml}$ tubes to approximately $1 \mathrm{mg} / \mathrm{ml}$ VGLUT1 and 1\% DDM ( 20fold). The concentrators would not allow for the majority of the DDM (micellar size $\sim 50 \mathrm{kDa}$ ) to pass freely and therefore a final concentration of $1 \%$ DDM is estimated on the assumption that the detergent is completely retained on the cisside of the concentrator membrane together with the transporter.

The concentrated sample was aliquoted in $20 \mu \mathrm{l}$ portions, snap-frozen in liquid $\mathrm{N}_{2}$ and stored at $-80^{\circ} \mathrm{C}$. For each expression the final sample was evaluated on $10 \%$ SDS-PAGE gels with Coomassie Blue staining for purity. The Coomassie stain also served to estimate the variation of protein concentrations among different expression experiments. The expression was found to be very reproducible yielding repeatedly 200-400 $\mu \mathrm{g}$ of purified VGLUT1 from 100 dishes ( 3-4 L of medium consumed in total). 
All other isoforms (DVGLUT, eat-4, VGLUT2) were expressed in essentially the same way. See 2.10.3 for details of the DNA-constructs.

\subsection{Reconstitution of a proton pump and VGLUTs into artificial membranes}

Proteoliposomes were formed by detergent removal after mixing of detergent-solubilized components, namely phospholipids, cholesterol and membrane proteins, with dialysis.

Soybean phospholipids (type II, Sigma) were solubilzed at $40 \mathrm{mg} \mathrm{ml}^{-1}$ in $5 \% \mathrm{OG}$ and stored at $-80^{\circ} \mathrm{C}$. Cholesterol was solubilzed in $7 \%$ OG. For cholesterol it was necessary to heat the suspension for $\sim 1$ hour to $50^{\circ} \mathrm{C}$, otherwise solubilization was not complete. The cholesterol-stock was stored at $25^{\circ} \mathrm{C}$. Phospholipids were mixed with cholesterol to reach a molar content of $\sim 25 \%$ for cholesterol. For this an average MW of $\sim 800 \mathrm{Da}$ was assumed for the phospholipid components. Lipids and proteins were mixed at a ratio (wt/wt) of $\sim 50$ for $\mathrm{TF}_{0} \mathrm{~F}_{1}$ and $\sim 25$ for VGLUT1. This resulted in $\sim 5-10$ copies of VGLUT1 / $\mathrm{TF}_{0} \mathrm{~F}_{1}$. When VGLUT1 was ommitted, the same volume of the corresponding elution buffer (see 2.4.2) containing $1 \%$ DDM (wt/vol) was added instead. The final lipid concentration was adjusted to $2.5 \mathrm{mg} \mathrm{ml}^{-1}$ with buffer (same as dialysis buffer). First the lipids were mixed, then buffer was added and finally the proton pump and the transporter were added to avoid exposure of the proteins to high concentrations of OG.

The slightly turbid solution was dialysed (12-14 kDa cutoff, SpectraPor) over night against $5 \mathrm{mM}$ MOPS-K (pH 7.3), $2 \mathrm{mM} \mathrm{MgSO}_{4}$ and $100 \mathrm{mM}$ of either potassium gluconate (KGluc) or $\mathrm{KCl}$ to determine the internal anion of the liposomes. Intermediate concentrations of $\mathrm{Cl}^{-}$were achieved by mixing KGluc- and $\mathrm{KCl}$ based buffers accordingly. Up to $5 \mathrm{ml}$ of liposome premix were dialysed in $1 \mathrm{~L}$ of buffer to efficiently remove the OG and from liposomes. The dialysis buffer was substituted with 1 teaspoon of BioBeads (BioRad) to adsorb OG-monomers and thus aid in removal of the detergent. After dialysis, DDM was complexed by addition of a 2-fold molar excess of solid 2,6-di-O-methyl- $\beta$ cyclodextrin ( $\beta$-CD) (Degrip et al., 1998). The liposomes were left on ice for $\sim 1$ hour before they were used. 


\subsection{Measurement of vesicle acidification with acridine orange (AO)}

Acridine orange is a fluorescent DNA-binding dye. Upon DNA- (or RNA-) binding its fluorescence is enhanced. However it fluoresces also in the absence of nucleic acids. The absorption maximum is at $492 \mathrm{~nm}$ and the emission maximum is at $530 \mathrm{~nm}$ wavelength. It is a weak base, the property which was used in this study. In its unprotonated form it can pass lipid membranes, but upon protonation (e.g. in an acidic interior of a vesicle) it becomes positively charged and thus trapped in the lumen. The accumulation of AOmolecules in the lumen leads to self-quenching which reduces the fluorescent emission. This reduction in the emission (or absorbance) is a readout for the acidification of vesicle lumina. The dye responses very fast and has been widely used for the measurement of organelle acidifcation (Maycox et al., 1988; Tabb et al., 1992) by changes in fluorescence or absorbtion in fluoro- or photometers, respectively. Acridine orange is available from Molecular Probes and was stored in $30 \%$ ethanol at a concentration of $200 \mu \mathrm{M}$ at $-20^{\circ} \mathrm{C}$. It should be noted that $\mathrm{AO}$ is not a $\mathrm{pH}$ indicator. It is only the trapping in vesicle lumina that leads to changes in the measured signal.

\subsubsection{Measurement of KCl- and KGlu-dependent acidification of LP2-fractions}

To measure the acidification of SVs (LP2), 20-60 $\mu \mathrm{g}$ LP2 sample from WT or KO mice were analysed in $2 \mathrm{ml}$ plastic or quartz cuvettes in a Hitachi F-2500 fluorometer under constant stirring at $32{ }^{\circ} \mathrm{C}$ with excitation and emission wavelengths of 492 and $530 \mathrm{~nm}$ respectively. The slit width for excitation and emission was $2.5 \mathrm{~nm}$ and the high voltage was set to $700 \mathrm{~V}$. Data was integrated in 1-2 s intervals.

LP2 was suspended in $1 \mathrm{ml}$ of acidification buffer ( $0.3 \mathrm{M}$ sucrose, $4 \mathrm{mM} \mathrm{MgSO}, 2 \mu \mathrm{M}$ acridine orange, $10 \mathrm{mM}$ MOPS-K, $\mathrm{pH}$ 7.3) in the cuvette. For the measurement of $\mathrm{Cl}^{-}$ dependent acidification, $\mathrm{KCl}$ was added to a final concentration of $100 \mathrm{mM}$ from a $3 \mathrm{M}$ stock solution and for glutamate dependent acidifcation potassium glutamate (KGlu) and $\mathrm{KCl}$ were added to 10 and $4 \mathrm{mM}$ respectively. $\mathrm{KGlu}$ was stored as $1 \mathrm{M}$ stock at $-20^{\circ} \mathrm{C}$ with a $\mathrm{pH}$ of 7.3. After 3 minutes of incubation the recording was started. 30-60 seconds later ATP was added to a final concetration of $2 \mathrm{mM}$ (from a $120 \mathrm{mM} \mathrm{K}$-ATP stock, adjusted to $\mathrm{pH}$ 7.3) by pipetting into the cuvette while recording. The addition of ATP led to an 
immediate rise in the signal because the fluorescence of $\mathrm{AO}$ is greatly enhanced by nucleotides. This jump was later subtracted (not in all figures) for presentation purposes and to normalize all traces to the same starting point. A gradual reduction in fluorescence over time indicated the acidifcation of vesicle lumina in the suspension. If no acidifcation occurred, the AO signal remained at the level after ATP addition. The sample was measured for $\sim 10$ minutes and the reaction was terminated by the addition of the proton uncoupler carbonyl cyanide $p$-(trifluoromethoxy)phenylhydrazone (FCCP) at a final concentration of $40 \mu \mathrm{M}$. The deacidifcation by FCCP addition led to an increase of the signal, almost to the level after the addition ATP. The deacidifcation was markedly slower for KGlu induced acidification. Since the fluorescence signal drifted slightly during the measurement ( 100 AU decrease, probably by adsorption of the dye on the cuvette walls) a baseline was recorded without the addition of ATP, but including the addition of FCCP. This baseline was later subtracted from all measurements.

The acidification of an experiment was quantified by taking the difference in AO fluorescence $10 \mathrm{~s}$ after the addition of ATP (to ensure mixing and thus a stable value) and $10 \mathrm{~s}$ prior the addition of FCCP. The mean of three measurements was taken for the presentation of traces if not indicated differently.

\subsubsection{Measurement of $\mathrm{KCl}$-dependent acidification of reconstituted vesicles}

The acidification of liposomes was usually measured with gluconate as the internal anion but was also tested with acetate or $\mathrm{Cl}^{-}$as internal anions. Typically, $50 \mu \mathrm{l}$ of liposomes (125 $\mu \mathrm{g}$ lipids, $2.5 \mu \mathrm{g} \mathrm{TF}_{0} \mathrm{~F}_{1}$ and $5 \mu \mathrm{g}$ VGLUTs) were added to $1 \mathrm{ml}$ of $100 \mathrm{mM}$ potassium gluconate, $2 \mathrm{mM} \mathrm{MgSO}_{4}$, and $5 \mathrm{mM}$ MOPS-K, pH 7.3 (dialysis buffer). $\mathrm{KCl}$, AO, ATP and FCCP were added as described in 2.6.1. Valinomycin (VAL) was added to a final concentration of $5 \mathrm{nM}$. The data were quantified as described in 2.6.1.

\subsection{Measurement of glutamate uptake}

Glutamate uptake of reconstituted liposomes was meausured with the use of radiolabelled glutamic acid $\left(\left[{ }^{3} \mathrm{H}\right]\right.$-glutamic acid, GE). The standard uptake was measured at $32^{\circ} \mathrm{C}$ with final concentrations of $4 \mathrm{mM}$ ATP, $40 \mu \mathrm{M}$ potassium glutamate $10 \mathrm{mM}$ potassium 
aspartate, $4 \mathrm{mM} \mathrm{KCl}$ and $2 \mu \mathrm{Ci}\left[{ }^{3} \mathrm{H}\right]$-glutamic acid per data point, if not indicated differently in the text or figures. The reaction was started by addition of prewarmed liposomes to a 10x reaction mixture and stopped by flushing 200-600 $\mu 1$ (depending on the experimental purpose) into $4 \mathrm{ml}$ of ice-cold uptake buffer (100 mM KGluc, $2 \mathrm{mM} \mathrm{MgSO} 4$ and $5 \mathrm{mM}$ MOPS-K, $\mathrm{pH}$ 7.3). The liposomes were then filtered through nitrocellulose membranes by vacuum and washed 3 times with $4 \mathrm{ml}$ of ice cold uptake buffer. After filtration the trapped radioactivity was counted by liquid scintillation. Nigericin (NIG), VAL, FCCP and Evan's Blue (EB) were added to the 10x reaction mixture where indicated to reach final concentrations of $200 \mathrm{nM}, 100 \mathrm{nM}, 20 \mu \mathrm{M}$ and $1 \mu \mathrm{M}$ respectively. $10-20 \mu \mathrm{g}$ VGLUT were assayed per data point.

\subsubsection{Control of the intra- and extravesicular ion composition for glutamate uptake experiments}

The internal anion was set by the dialysis buffer and contained $100 \mathrm{mM}$ gluconate or $\mathrm{Cl}^{-}$as the major anions. Intermediate concentrations were achieved by mixing buffers in the desired ratio. After addition of $\beta-\mathrm{CD}$ and incubation on ice, 1-2 $\mathrm{ml}$ of the liposomes were passed through a self-made Sephadex 25 (GE) gel filtration column to exchange the external anion. Gluconate loaded liposomes were also passed through the column. The columns were made from maxi-Prep columns for the purifcation of DNA. The original resin was removed and a wet bed of 10-20 ml Sephadex 25 beads was layered on top of a frit. The gel-bed was secured on top with another frit that was gently pressed onto the beads. The bed volume was sufficient to separate small molecules or ions from high molecular weight proteins or vesicles (see Fig 3.30) and thus ensured the complete removal of $\mathrm{Cl}^{-}$from the extravesicular space. The liposomes were eluted with uptake buffer and $\mathrm{KCl}$ was then added to the reaction mix as desired. Only for measurements in the presence of $50 \mathrm{mM}$ external $\mathrm{Cl}^{-}$a modified elution buffer was used to avoid osmotic stress to the membranes. The fractions were collected in fractions of $1 \mathrm{ml}$. The liposome containing fractions were easily identified by their turbid appearance, pooled and immediately used for uptake experiments. Equal protein amounts after such a gelfiltration were tested by western blotting with anti-VGLUT1 antibodies. It was found that the method was accurate enough and equal volumes of liposomes contained equal amounts of transporter. 


\subsection{Electron microscopy}

Liposomes, prepared in gluconate or $\mathrm{Cl}^{-}$conatining buffers, were examined under the electron microscope to test whether they show similar diameters by negative staining. The solution containing liposomes in appropriate, experimentally determined dilutions, were applied on glow-discharged collodion-coated nickel-grids and fixed with $4 \%$ paraformaldehyde and $0.5 \%$ glutaraldehyde in phosphate buffer $(\mathrm{pH} 7.4)$. The grids were washed with phosphate buffer and then distilled water for staining with $1.5 \%$ uranyl acetate $(\mathrm{w} / \mathrm{v})$. The grids were observed and imaged in a Hitachi H-7100 electron microscope at $75 \mathrm{kV}$, equipped with a CCD (Hammatasu).

For the estimation of the liposome volumes, the mean diameter was determined by measuring the longest and shortest axis of a liposome (using the free ImageJ Software, NIH). From the mean diameter the volume of individual liposomes was calculated. A total of 600 liposomes from 10 micrographs was analyzed for both liposome types.

\subsection{Entrapment of $\left[{ }^{14} \mathrm{C}\right]$-glucose in liposomes for the determination of the intra- vesicular volume}

To estimate the volume of gluconate or $\mathrm{Cl}^{-}$loaded liposomes with a technique other than electron microscopy, a radiotracer experiment was devised. Two $\mathrm{ml}$ of $\mathrm{TF}_{0} \mathrm{~F}_{1}$-VGLUT1liposomes were formed in $500 \mathrm{ml}$ of buffer containing either $\mathrm{Cl}^{-}$or gluconate as the major anions. $25 \mu \mathrm{Ci}$ of $\left[{ }^{14} \mathrm{C}\right]$-glucose (GE) were added to the liposome premix prior to dialysis and the sample was dialyzed overnight at $4{ }^{\circ} \mathrm{C}$. Then the turbid suspensions were applied to gel filtration on Sephadex 25 columns (see also 2.7.1) and fractions of $1 \mathrm{ml}$ were collected. The radioactivity of the fractions was measured by liquid scintillation counting. The isotope was eluting in two clearly separated peaks (Fig. 3.30), of which the first peak contained the expected liposomes (the fractions were also turbid). This separation into two peaks was only possible if the radiotracer was trapped in the artificial vesicles during the formation process. The measured radioactivity in the liposome fraction is linearly proportional to the volume. Hence, half the radioactivity is related to only half the volume. 


\subsection{Miscellaneous general methods}

\subsubsection{Protein measurement, electrophoresis and immunoblotting}

Protein concentrations were measured with the BCA-Kit (Pierce). For very low concentrations the reaction was done at $60^{\circ} \mathrm{C}$ according to manufacturers instructions.

SDS-polyacrylamide gel electrophoresis (SDS-PAGE) was carried out according to Laemmli (Laemmli, 1970). Samples were mixed with 2x-4x SDS-sample buffer containing $\beta$-mercaptoethanol (1:10) and loaded without boiling on 7.5-15\% mini-gels (BioRad). After electrophoresis the gels were stained with Coomassie Blue (1.5\% Coomassie Brilliant Blue, 50\% methanol, $10 \%$ acetic acid) or by silver staining (Wako silver staining kit).

For immunoblotting the unstained gels were further electrophoresed by semidry-transfer using standard procedures (Towbin et al., 1979). The transfer on PVDF membranes $($ Schleicher + Schuell) was done at $15 \mathrm{~V}$ for 30 minutes with $100 \mathrm{mM}$ Tris and $20 \%$ methanol, pH 11 according to manufacturers instructions. The PVDF membranes were blocked with $5 \%$ skim milk in 1x TBS, $0.1 \%$ Tween-20 (blocking buffer) and then probed with specific and secondary antibodies (see 2.10.2) in blocking buffer for $~ 30-60$ minutes at $25^{\circ} \mathrm{C}$ or overnight at $4{ }^{\circ} \mathrm{C}$. The membranes were washed between primary and secondary antibodies and before detection 3 times for $\sim 5$ minutes with TBS, $0.1 \%$ Tween20. For detection with the HRP-conjugated secondary antibodies the membranes were wetted with ECL-substrate (PE) and imaged in a BioRad ChemidocXRS-J dark box (BioRad) with a cooled CCD.

\subsubsection{Antibodies}

The rabbit polyclonal antisera to VGLUT1, VGLUT2, VIAAT, VATPase a-subunit (pp116) and GFP were kindly donated by Prof. Reinhard Jahn (Max-Planck-Institut für biophysikalische Chemie, Göttingen) and are commercially available from Synaptic Systems GmbH (Göttingen) (see www.sysy.com for details). VGLUT1, VGLUT2 and VIAAT antibodies were affinity purified with the corresponding bead-immobilized antigens (GST-fusion proteins for VGLUT1/2 and an antigenic peptide for VIAAT). 
Synaptophysin (Syp) was detected with the mouse monoclonal antibody Cl 7.2 (Synaptic Systems) also donated by Prof. Reinhard Jahn as well as the monoclonal anti-myc antibody (MYC, Synaptic Systems).

The guinea-pig anti-VGLUT1 antibody was donated by T. Kaneko (University of Kyoto) (Fujiyama et al., 2001). Affinity purified rabbit polyclonal antibody to $\mathrm{rClC}-3$ was from Alomone Labs. The affinity purified rabbit antibody to DVGLUT was a kind gift of A. DiAntonio (University of Washington, St. Louis, Missouri) (Daniels et al., 2004). Antibodies were used at dilutions of 1:5000 (VGLUT1, DVGLUT, Syp), 1:3000 (VGLUT2, myc), 1:1000 (VIAAT, pp116) and 1:200 (ClC-3)

HRP-conjugated anti-rabbit, anti-mouse or anti-guinea pig antibodies were from BioRad and used at dilutions of 1:5000-1:10000.

\subsubsection{Molecular cloning}

The DNA constructs were generated by standard molecular biology techniques using the polymerase chain reaction (PCR), restriction endonucleases and T4-ligase (Sambrook et al., 2001). The cDNA for rVGLUT1 (Takamori et al., 2000a) was PCR-amplified from a pCDNA3.1 expression vector (Invitrogen) and fused C-terminally to the SBP-tag connected by a TEV-linker into pBluescript SK+ (Stratagene). The SBP-tag was PCRamplified from the plasmid pTAG2K (Keefe et al., 2001) which was kindly donated by J.W. Szostak (Harvard University, Boston). The transporter gene was cloned with NheI (5'-end) and XhoI (3'-end) 3' of the SBP tag. The 5'-untranslated region of the human vascular endothelial growth factor (VEGF) called SP163 (PCR-amplified from pcDNA4HisMax, Invitrogen) was positioned upstream of the SBP tag and terminated with a Kozak sequence and the start codon. This cassette served also for all isoforms that were expressed. For this, the rVGLUT1 gene was excised with NheI and XhoI and the vector was purified by agarose gel electrophoresis. Then the NheI/XhoI-digested PCR-products of other cDNAs were ligated into the vector by T4-Ligase (Invitrogen). For shuttling to pCDNA3.1, the entire expression cassette of about $\sim 2 \mathrm{kbp}$ was excised with EcoRI (5'end) and XhoI, XbaI or NotI, gel purified and ligated into the multiple cloning site (MCS) of pcDNA3.1. The cassette with the most important restriction sites is shown in figure 2.2. 
Mouse VGLUT2 cDNA was amplified from a mouse brain cDNA library by Dr. Mitsuko Hirosawa-Takomori and then PCR-amplified for subsequent cloning steps. DVGLUT

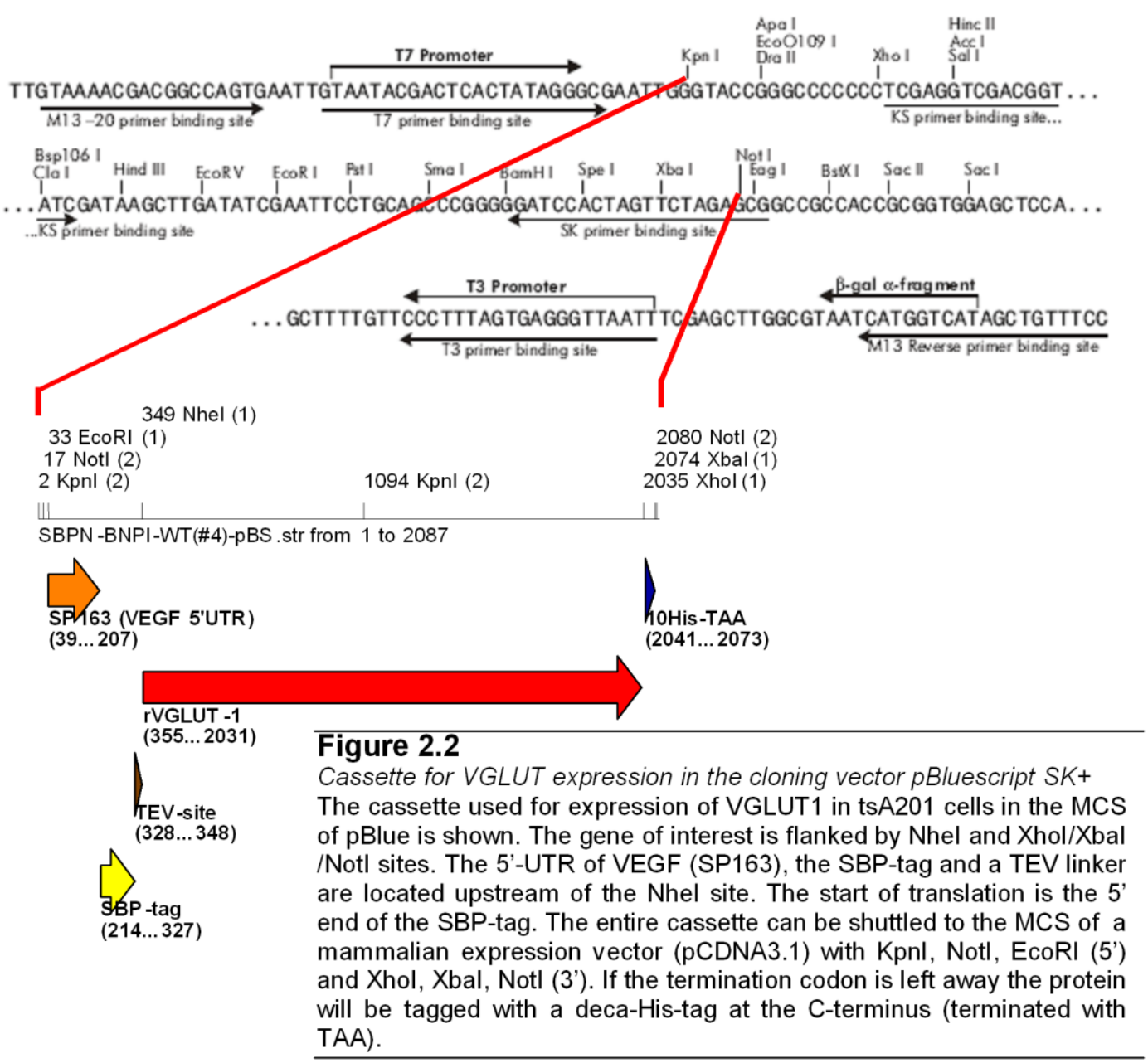

(CG9887) cDNA was PCR-amplified from the D. melanogaster EST-clone 5477 donated by A. DiAntonio (Washington University, St. Louis, Missouri) and eat-4 was PCRamplified from the EST-clone pPD49.26, donated by Ikue Mori (Nagoya University). Since eat-4 is cleaved by EcoRI the NheI/XhoI digested cDNA was directly ligated into a prepared pCDNA3.1 vector. The first $63 \mathrm{bp}$ of the eat-4 ORF were exchanged with the myc-tag.

For an N-terminal fusion of the myc peptide, VGLUT1 was cloned into the pBob vector (a derivative of pCDNA 3.0 by T.C. Südhof, Stanford University) by Dr. Shigeo Takamori. VGLUT1-Venus was cloned into pCDNA3.1 (PCR-amplified without the stop codon) omitting the above mentioned expression cassette with the SBP tag. Instead the native start 
codon was used and Venus-YFP (donated by A. Miyawaki, BSI-RIKEN, Wako-shi) was fused at the 3'-end with a connecting HindIII site. VGLUT1-VenusYFP was cloned with

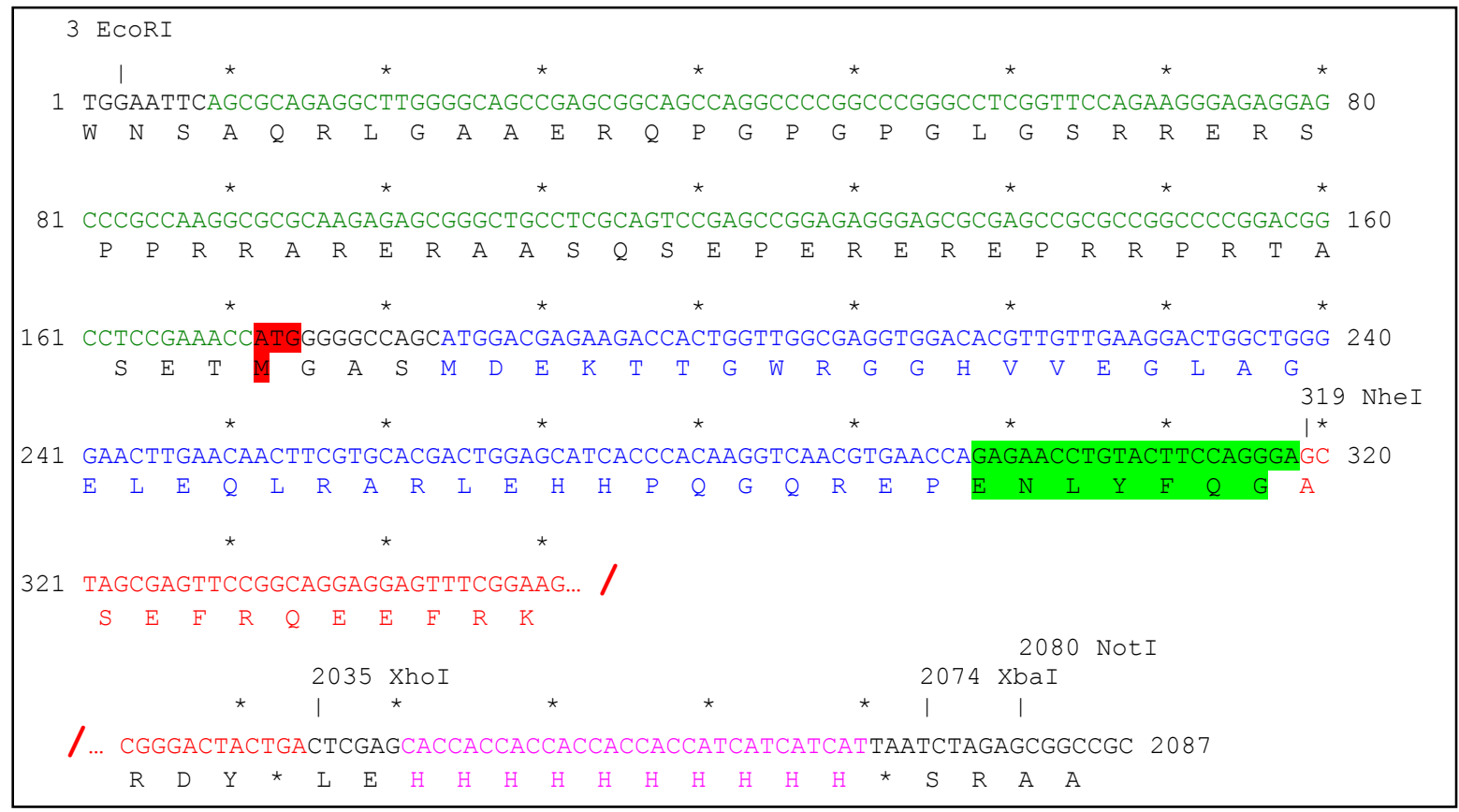

\section{Figure 2.3}

Sequence details for the cloning of SBP-tagged VGLUT genes

DNA sequence of flanking regions upstream and downstream of the VGLUT1 gene. SP163 (green), the SBP-tag (blue), the TEV-cleavage site (black on green), rVGLUT1 (red) and the deca-His tag (pink) are highlighted. The start codon is marked with black characters on red ground. Cloning without the termination codon prior to the Xhol site results in a transporter with an additional C-terminal His-tag. All transporters under investigation were cloned with this scheme.

XhoI 5' and 3'. Human ClC-3 cDNA was donated by T.J. Jentsch (Leibniz-Institut, MDC, Berlin) and cloned into pcDNA3.1 as an EGFP-fusion protein by Dr. Shigeo Takamori.

Single amino acid substitutions were introduced into rVGLUT1 (in pBlue SK+) by site directed mutagenesis using the Quickchange mutagenesis kit (Stratagene), following the instruction manual. The primers used to introduce the mutations are shown in table 1. 


\begin{tabular}{|c|c|c|}
\hline $\begin{array}{c}\text { Amino acid } \\
\text { change / name }\end{array}$ & Sequence $\left(\mathbf{5}^{\prime} \rightarrow \mathbf{3}^{\prime}\right)$ & ') \\
\hline $\mathrm{H} 120 \mathrm{~A} / 210$ & CTGTCGGCCTCATAGCTGGCTCCTTTTTCTGG & forward \\
\hline $\mathrm{H} 120 \mathrm{~A} / 211$ & ATGTACCCCCAGAAAAAGGAGCCAGCTATGAGGCC & reverse \\
\hline $\mathrm{R} 176 \mathrm{E} / 208$ & GGCTGTGTCATCTTCGTGGAGATCCTTCAGG & forward \\
\hline $\mathrm{R} 176 \mathrm{E} / 209$ & CCACCAATCCCTGAAGGATCTCCACGAAGATG & reverse \\
\hline $\mathrm{R} 166 \mathrm{~T} / 358$ & TTGATCCCTTCAGCAGCAGCCACTGTTCACTATGGC & forward \\
\hline $\mathrm{R} 166 \mathrm{~T} / 359$ & GATGACACAGCCATAGTGAACAGTGGCTGCTGAAG & reverse \\
\hline $\mathrm{K} 339 \mathrm{E} / 360$ & GTTCGGCTTTGAGATCAGCGAGGTGGGACTGGTG & forward \\
\hline $\mathrm{K} 339 \mathrm{E} / 361$ & TGCCGACACCAGTCCCACCTCGCTGATCTCAAAG & reverse \\
\hline $\mathrm{R} 368 \mathrm{~N} / 362$ & CGCCGACTTCCTGCGCAGTAATCATATAATGTCC & forward \\
\hline $\mathrm{R} 368 \mathrm{~N} / 363$ & TGGTCGTGGACATTATATGATTACTGCGCAGGAAG & reverse \\
\hline $\mathrm{K} 401 \mathrm{~A} / 364$ & GGTCGGATACTCACACTCCGCGGGCGTGGCCATC & forward \\
\hline $\mathrm{K} 401 \mathrm{~A} / 365$ & GGAAGGAGATGGCCACGCCGCGGGAGTGTGAGTATC & reverse \\
\hline $\mathrm{K} 463 \mathrm{~N} / 366$ & GGGTGCAATGACCAAGCACAATACGCGGGAGGAG & forward \\
\hline $\mathrm{K} 463 \mathrm{~N} / 367$ & TACTGCCACTCCTCCCGCGTATTGTGCTTGGTCATTG & reverse \\
\hline
\end{tabular}

Table 1: Primer sequences for the introduction of single amino acid substitutions in rVGLUT1 by site directed mutagenesis

All cloning steps as well as plasmid preparation in small and large scale were done using chemically competent XL-1 Blue E. coli cells.

\subsubsection{Genotyping}

Mice were genotyped by PCR with template DNA from mouse tail biopsies according to the original description of the $\operatorname{Vglut} \mathrm{I}^{-/}$and $\mathrm{Clcn}^{-/-}$strains (Wojcik et al., 2006; Yoshikawa et al., 2002). About $0.5 \mathrm{~cm}$ of mouse tail tips were digested in $500 \mu \mathrm{l}$ SNET-buffer ( $1 \%$ SDS, $400 \mathrm{mM} \mathrm{NaCl}, 25 \mathrm{mM}$ EDTA, $50 \mathrm{mM}$ Tris , $\mathrm{pH}$ 8.0) containing $1 \mathrm{mg} / \mathrm{ml}$ Proteinase $\mathrm{K}$ at $52{ }^{\circ} \mathrm{C}$ overnight. $500 \mu \mathrm{l}$ of buffer saturated phenol (Invitrogen) were added to each sample and gently mixed for 3 minutes before centrifugation at 15,000 rpm for 5 minutes on a table top centrifuge. The upper aqueous phase was transferred to fresh tubes and 500 $\mu 1$ chloroform were added. The samples were mixed for 3 minutes and then spun again at 15,000 rpm for 5 minutes. The upper phase was mixed in a new tube with $1 \mathrm{ml}$ of absolute ethanol and spun at 15,000 rpm for $10 \mathrm{~min}$. The solution was removed from the DNApellet which was then dried for 30 minutes at $25^{\circ} \mathrm{C}$. $250-400 \mu 1$ of TE buffer (10 mM Tris- 
Cl, 1 mM EDTA, $\mathrm{pH}$ 8.0) were added to dissolve the dry DNA at least for 30 minutes. 1-2 $\mu 1$ were then used in the PCR reaction which is identical for Vglut1 and Clcn3 mouse strains except for the specific primers (primer sequences are listed in table 2). The composition for one reaction $(20 \mu \mathrm{l})$ was as follows :

$\begin{array}{lr}\text { 10x Buffer (with KCl) } & 2 \mu 1 \\ \text { dNTP mix }(2.5 \mathrm{mM}) & 1 \mu \mathrm{l} \\ \text { Primer \# } 1(5 \mu \mathrm{M}) & 1 \mu \mathrm{l} \\ \text { Primer \# 2 }(5 \mu \mathrm{M}) & 1 \mu \mathrm{l} \\ \text { Primer \# 3 }(5 \mu \mathrm{M}) & 1 \mu \mathrm{l} \\ \text { Taq (LC /Fermentas 1u/ } \mu \mathrm{l}) & 1 \mu \mathrm{l} \\ \mathrm{MgCl}_{2}(25 \mathrm{mM}) & 0.9 \mu \mathrm{l} \\ \mathrm{Genomic} \mathrm{DNA} & 1-2 \mu \mathrm{l} \\ \text { Water } & 10.1-11.1 \mu \mathrm{l}\end{array}$

\begin{tabular}{|c|c|c|}
\hline Name & Mouse strain & Sequence $(\mathbf{5} \rightarrow \mathbf{3}$ ') \\
\hline Neo AN / 161 & Clcn3 & TCCTGACTAGGGGAGGAGTAGAAGTG \\
\hline mCLC3-wild / 162 & Clcn3 & TGCTTTTAACTACTGTGCTATTGC \\
\hline mCLC-3-shortArm1 /163 & Clcn3 & TGTGACCATAGTAAGTACCTC \\
\hline 4172 & Vglut1 & TTCAGCAGCCCGCGTTCACTATGG \\
\hline 4173 & Vglut1 & CCCAGCTCAGCCCTCCTCGCACAA \\
\hline 4174 & Vglut 1 & CGCATCGCCTTCTATCGCCTTCTT \\
\hline
\end{tabular}

Table 2: Primer sequences for the genotyping of Vglut1 and Clcn3 mouse strains 


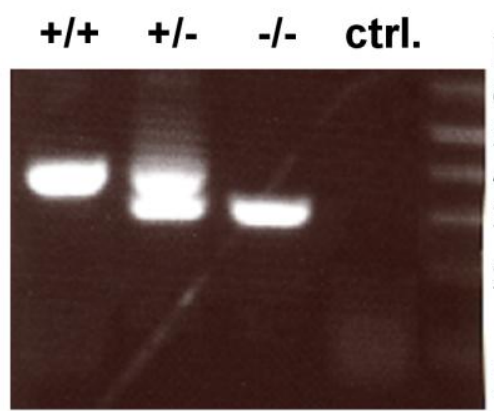

Vglut1

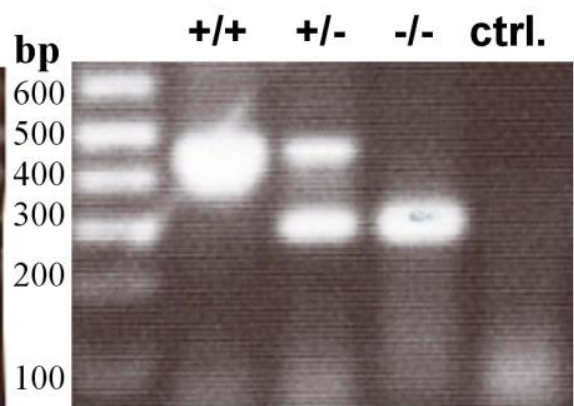

Clcn3

\section{Figure 2.4}

Genotyping of Vglut 1 and Clcn3 mouse strains

PCR products visualized in $1.5 \%$ agarose gels stained with ethidium bromide. Genomic DNA from wild type, heterozygous and knock out animals was amplified with specific primers (see table 2) by PCR. The 100 bp-ladder is loaded on the right for Vglut1 and on the left for Clcn3.

The PCR profile was $95^{\circ} \mathrm{C}$ for 5 minutes followed by 40 cycles of $95{ }^{\circ} \mathrm{C}$ for 45 seconds, $60^{\circ} \mathrm{C}$ for 30 seconds and $72{ }^{\circ} \mathrm{C}$ for 45 seconds. $15 \mu$ were analyzed on $1.5 \%$ agarose gels. The Vglut1 WT allele yields a $280 \mathrm{bp}$ amplicon and the WT allele is $357 \mathrm{bp}$. For Clcn3 the WT and KO allele result in $\sim 300$ and $\sim 400$ bp respectively. A result for wild type, heterozygous and knock out cases is shown in figure 2.4. 


\section{RESULTS}

\subsection{Analysis of synaptic vesicles isolated from $\mathrm{Clcn3}^{-/-}$- and $\mathrm{Vglut}^{-/-}$-brains.}

The recently established mutant mice that lack ClC-3 (Stobrawa et al., 2001; Yoshikawa et al., 2002) and VGLUT1 (Fremeau et al., 2004; Wojcik et al., 2004) made it possible to directly compare the loss of either gene on the anion conductance of SVs. Therefore the two mutant strains were bred to get a sufficient number of knockout animals, necessary for the measurement of luminal acidfication. To investigate the contribution of VGLUT1 and ClC-3 proteins on $\mathrm{Cl}^{-}$shunting in SVs, a light membrane fraction enriched in SVs (LP2) was isolated by subcellular fractionation from whole brains of the corresponding mutant mice for comparison with wild type samples (see 2.2.1 for details). An influence of VGLUT1 on the chloride conductance in native material would imply also a role in vivo and thus justify a deeper investigation regarding the role of this conductance on the loading process of synaptic vesicles. Another central aim of this work was to measure the acidification of SVs from $\mathrm{Clcn}^{-/}$brains before the onset of neurodegeneration at the age of three weeks to shed light on the phenotype of the $\mathrm{Clcn}-3^{-/-}$mouse strain in order to get a plausible explanation for the observations made hitherto. The rationale behind this approach is simple albeit it was probably overlooked in previous studies: The brain regions in the $\mathrm{Clcn}^{-/-}$mice that are most affected by neurodegeneration overlap (not surprisingly) with the expression pattern of this protein (Kawasaki et al., 1994). Therefore, it is clear that the impact of the $\mathrm{ClC}-3$ gene loss can hardly be evaluated if the structure (SVs) where ClC-3 is supposedly located (and consequently would have an influence on the ion movements) has vanished (along with entire populations of neurons). If $\mathrm{ClC}-3$ would serve as a global shunt on SVs this should be notable also at a younger age and the preparations should be better suited for a comparison with the Vglutl $^{-/}$brains, which are morphologically much less influenced by the gene loss (Wojcik et al., 2004). 


\subsubsection{Measurement of the $\mathrm{Cl}^{-}$- and glutamate-dependent acidification of $\mathrm{LP2}$}

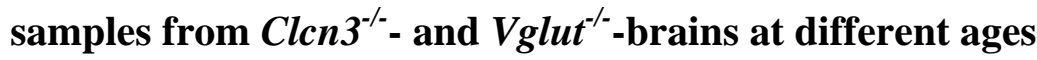

For both KO-strains the yields of SVs were notably smaller than for wild type mice. This was most obvious for brains of adult $\mathrm{Clcn}^{-/}$mice (approximately reduced by $50 \%$ to yields from wild type brains), a sign for the major impact on the brain morphology by the deletion of this gene. In order to standardize the result, the LP2-isolates were adjusted to the same protein level $(2.5 \mathrm{mg} / \mathrm{ml})$ and then checked for the relative abundance of some

\section{Clcn3}
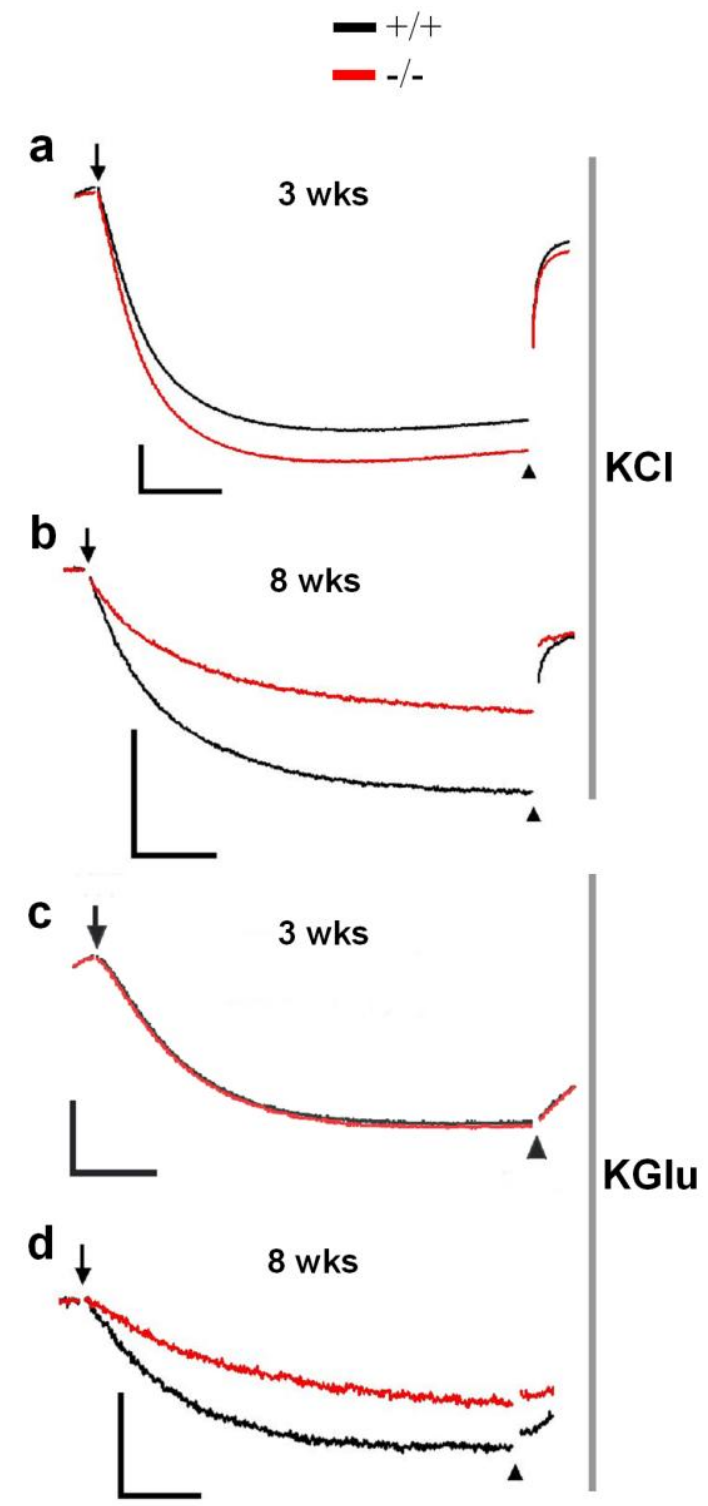

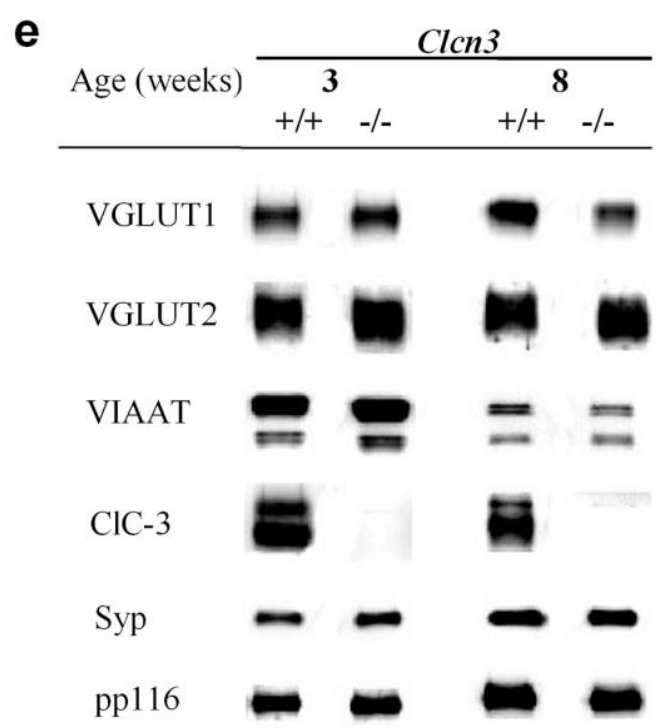

Figure 3.1

Acidification of SVs (LP2) from $\mathrm{Clcn}^{-1-}$ brains in the presence of external $\mathrm{C} \Gamma$ or glutamate. (a-d) Acidification of SVs from wild type (black) and $\mathrm{Clcn}^{-/-}$(red) mice at the age of 3 weeks ( $a$ and $c$ ) and 8 weeks ( $b$ and $d$ ) in the presence of $100 \mathrm{mM} \mathrm{KCl}$ ( $a$ and b) or $4 \mathrm{mM}$ $\mathrm{KCl}+10 \mathrm{mM} \mathrm{KGlu}$ (c and d) measured under standard conditions (see 2..6.1). A decrease in fluorescence reflects acidification. The traces represent the average of triplicate measurements. Scale bars represent $500 \mathrm{AU}$ and $120 \mathrm{~s}$. Arrows and arrowheads mark the addition of ATP and FCCP, respectively. (e) Representative immunoblots of relevant proteins in the SV fractions from $\mathrm{Clcn3}^{-1-}$ mice compared to wild type; pp116: V-ATPase asubunit. Note the reduced signal of VGLUT1 in samples from $\mathrm{Clcn}^{-/}$at the age of 8 weeks. See quantitative representations of the results in figs. 3.3 (acidification) and 3.4 (protein levels). 
relevant marker proteins by immunoblotting (Fig. 3.1e, 3.2e and 3.4a-d for a quantitative representation). Acidification of LP2 samples from mutant strains was compared to LP2 samples from wild type mice by fluorescence quenching of acridine orange (AO) (see 2.6.1).

It is evident that the loss of $\mathrm{ClC}-3$ does not cause a reduction in the $\mathrm{Cl}^{-}$dependent acidification of SVs when isolates of young mice (3 weeks of age) were investigated (Fig. 3.1a). Previous findings that were based on samples from old mice after severe
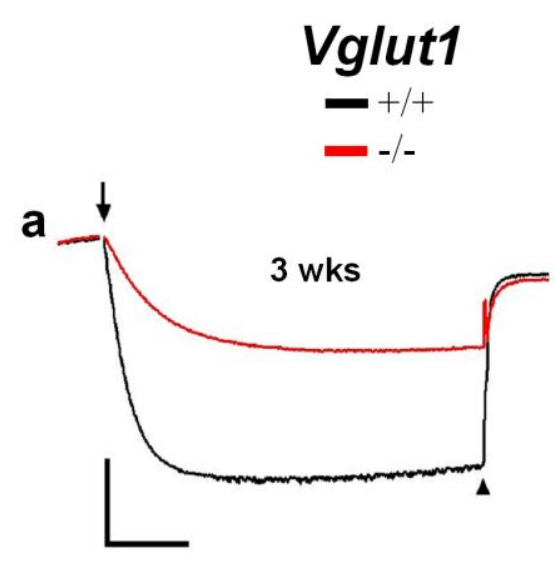

b
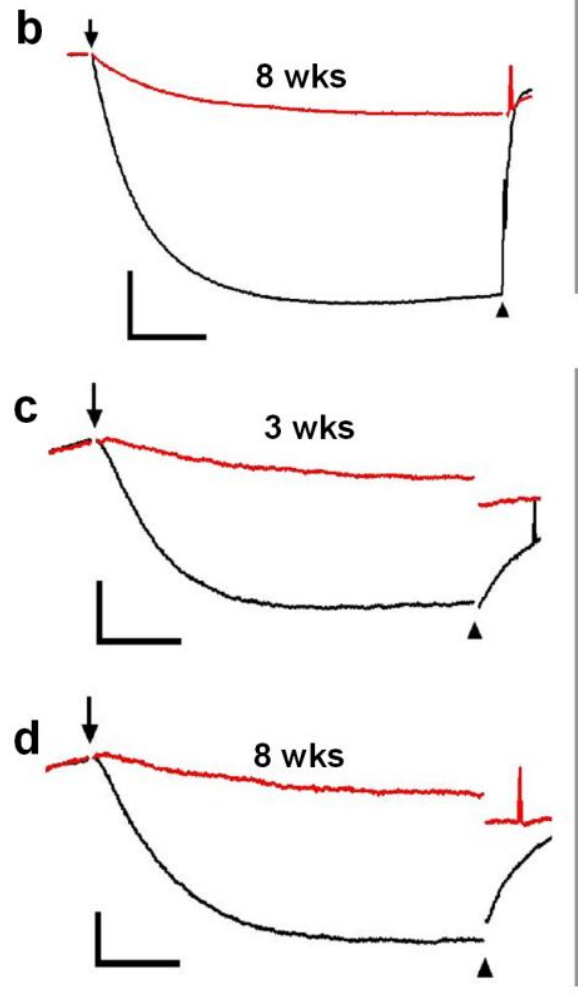

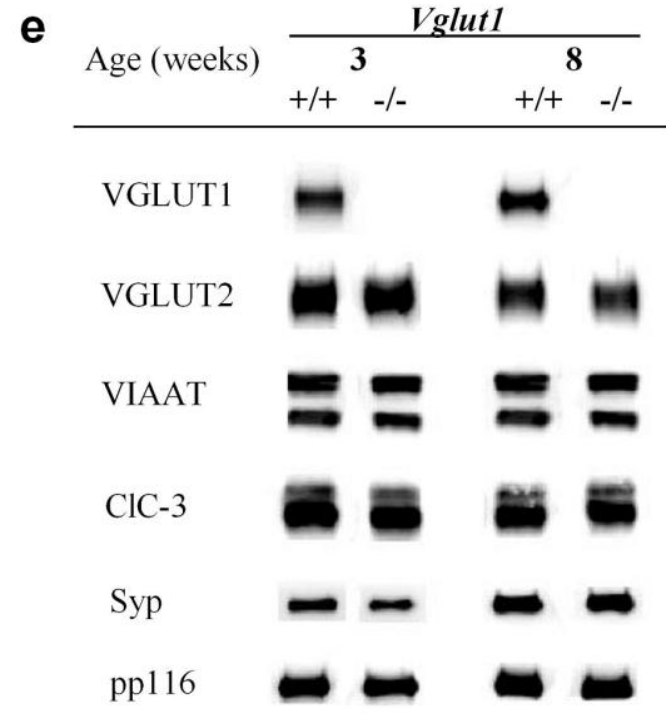

Figure 3.2

Acidification of SVs (LP2) from Vglut $1^{-1-}$ brains in the presence of external $\mathrm{C} \Gamma$ or glutamate. (a-d) Acidification of SVs from wild type (black) and Vglut $^{-/-}$(red) mice at the age of 3 weeks ( $a$ and $c$ ) and 8 weeks ( $b$ and $d$ ) in the presence of $100 \mathrm{mM} \mathrm{KCl}$ ( $a$ and $b$ ) or $4 \mathrm{mM}$ $\mathrm{KCl}+10 \mathrm{mM} \mathrm{KGlu} \mathrm{(c} \mathrm{and} \mathrm{d)} \mathrm{measured} \mathrm{under}$ standard conditions (see 2.6.1). A decrease in fluorescence reflects acidification. The traces represent the average of triplicate measurements. Scale bars represent $500 \mathrm{AU}$ and $120 \mathrm{~s}$. Arrows and arrowheads mark the addition of ATP and FCCP, respectively. (e) Representative immu-noblots of relevant proteins in the SV fractions from Vglut $1^{-/-}$mice compared to wild type; pp116: V-ATPase asubunit. Note that the CIC-3 signal is not reduced in samples from $\mathrm{Vglut}^{1 /-}$ mice at neither age. See quantitative representations of the results in Figs. 3.3 (acidification) and 3.4 (protein levels). 
neurodegeneration (6 months of age (Stobrawa et al., 2001)) could be confirmed by using adult $\mathrm{Clcn}^{-/-}$mice at the age of 8 weeks (Fig. 3.1c), where the hippocampal area is already macroscopically affected and appears as a cavity when a brain is cut (the author's personal observation and see also (Stobrawa et al., 2001; Yoshikawa et al., 2002). The phenotype observed for adult mice is also linked to a reduction of VGLUT1 (Fig 3.1d and

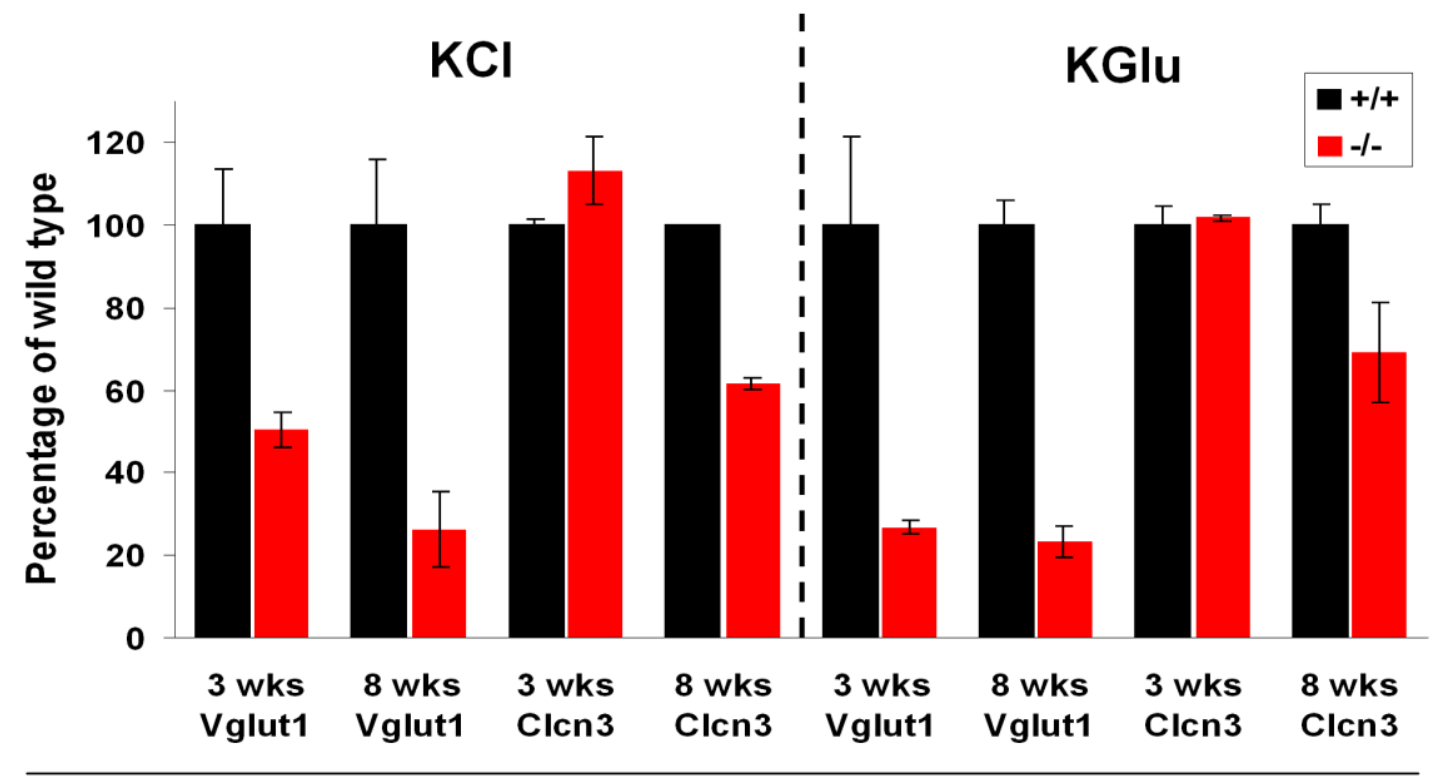

\section{Figure 3.3}

Quantitative summary of $\mathrm{Cr}$ - and glutamate-induced acidification of LP2 samples from VGLUT1- and CIC-3-deficient mice.

The decrease in fluorescence of $A O$ due to acidification of SVs was quantified using triplicate measurements (see 2.6.1 for details). On the left side of the panel $\mathrm{Cl}^{-}$ dependent acidification is shown and on the right side of the dashed line the glutamate dependent acidification.

3.4b), again reproducing previous results obtained with adult $\mathrm{Clcn}^{-1-}$ mice (Stobrawa et al., 2001). The deficits in $\mathrm{Cl}^{-}$dependent acidification for $\mathrm{SVs}$ from adult $\mathrm{Clcn}^{-/-}$mice were apparent also for glutamate dependent acidification (Fig. 3.1d), which requires low millimolar concentrations of $\mathrm{Cl}^{-}$to be effectively measurable (see 2.6.1 and Fig. 3.6a-b). When the glutamate dependent acidification was tested for SVs from young $\mathrm{Clcn}^{-/-}$mice, there was no obvious difference detectable (Fig.3.1c).

Notably, there is no reduction of VGLUT1 protein visible when LP2 fractions from young $\mathrm{Clcn}^{-1-}$ mice were probed (Fig. 3.1e, 3.4a). This is at least indicative for a relevant influence on the synaptic vesicle composition during aging in this mouse strain and coincides with the observation that the hippocampus is rich in VGLUT1-posititve terminals (Fremeau et al., 2001). 
Figure 3.4

Quantitative comparison of protein levels for relevant SV-proteins in SV fractions derived from brains of Vglut $1^{-1-}$ and $\mathrm{Clcn}^{-1-}$ mice. (a-d) Quantitative representation of the expression levels for the tested proteins in SV fractions from the corresponding knockout mice (red bars) (normalized to wild type levels which are shown as black bars). See representative blot captures in figs. 3.1 and 3.2. Equal amounts of LP2samples were loaded on SDS-PAGE Gels, transferred to PVDF membranes and probed with specific antibodies. See fig. 3.5 for further details.
a: Clcn3, 3 weeks
b: Clcn3, 8 weeks
c: Vglut1, 3 weeks
d: Vglut 1,8 weeks

a

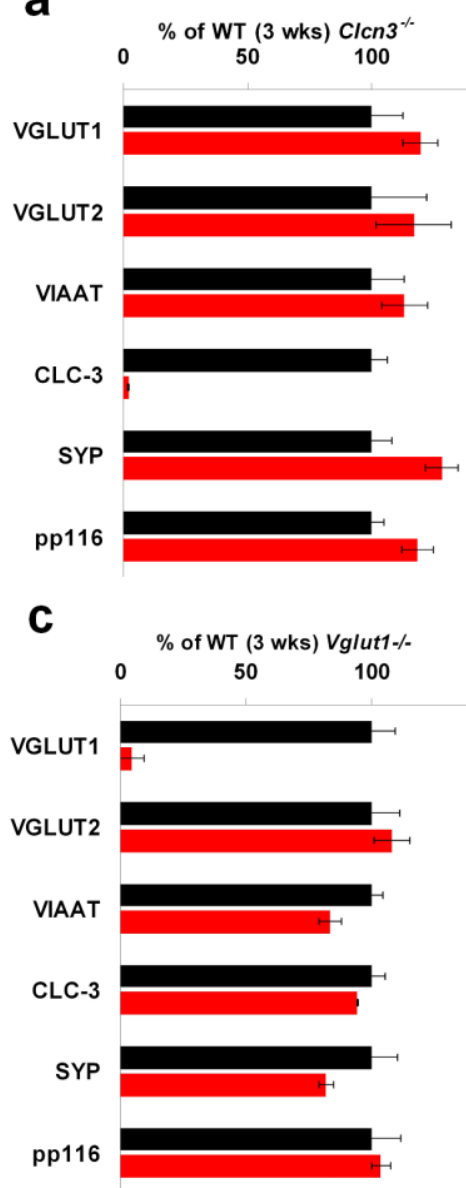

b

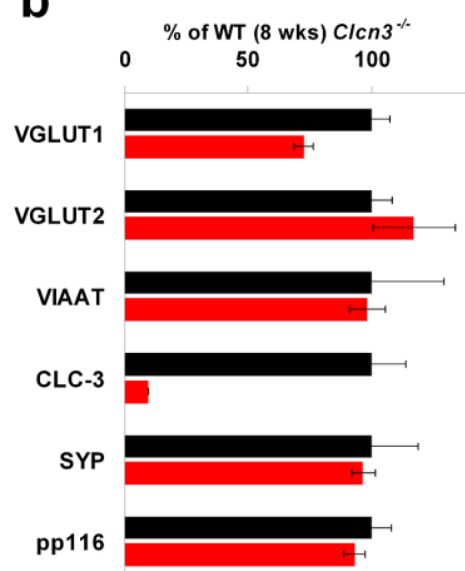

d

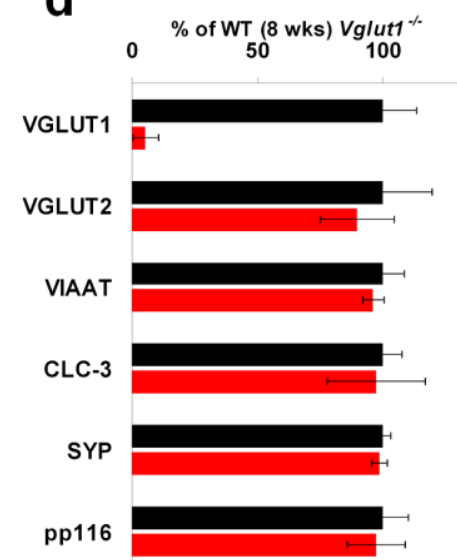

In strong contrast to the results with $\mathrm{Clcn}^{-/-}$mice there was a clear reduction of both, $\mathrm{Cl}^{-}$and glutamate dependent acidification measurable for SVs isolated from $\operatorname{Vglut}^{-/}$mice at the age of 3 weeks and 8 weeks (Fig. 3.2a-d). The remaining acidification in Vglut $^{-/}$ samples can be well explained with the fraction of SVs positive for VGLUT2 or the vesicular inhibitory amino acid transporter (VIAAT) (Takamori et al., 2000b). It should be noted here that there are also indications for a considerable spatial overlap of VGLUT2 expression on putative VGLUT1 type presynaptic terminals during the development of the brain (Boulland et al., 2004; Herzog et al., 2006) that might explain the somewhat lower impact of the gene loss on the acidification of vesicles from young $\operatorname{Vglut1}^{-/}$mice. Overall, no major changes in abundance for the proteins tested herein could be detected in LP2 fractions from $\mathrm{VGLUT1}^{-/-}$mice. Importantly, there was no decrease in the ClC-3 content (Fig. 3.2e, 3.4c-d). Since SV-markers like synaptophysin and the V-ATPase a-subunit did not show big variations between the tested samples, the material was well adjusted which allowed for a comparison in acidification by fluorescence measurement. 
The relative $\mathrm{Cl}^{-}$- and glutamate-dependent acidification of SVs from the two mutant strains compared to SVs from wild type mice is summarized in Fig. 3.3 (see 2.2.3 for details). The analysis of SVs from mutant mice clearly favored a requirement of VGLUT1 rather than $\mathrm{ClC}-3$ for the acidification of $\mathrm{SVs}$ from terminals that are positive for this transporter.

Figure 3.5
Calibration curves for the
antibodies and antigens used
to characterize LP2 samples.
Different amounts of samples
(LP2 fraction) were probed and
detected with HRP-conjugated
secondary antibodies (1:5000).
The red triangle on the
abscissa marks the amount of
sample which was used for
quantifications. All data in
figures $3.1,3.2$ and 3.4 have
been taken in the linear range
of the detection system.
Antibody dilutions were:
1:5000 (VGLUT1, Syp),
1:3000 (VGLUT2),
1:1000 (V-ATPase a-subunit,
VIAAT) and 1:200 (CIC-3).
The insets show the
corresponding blot image used
for the calibration curve.
(n)
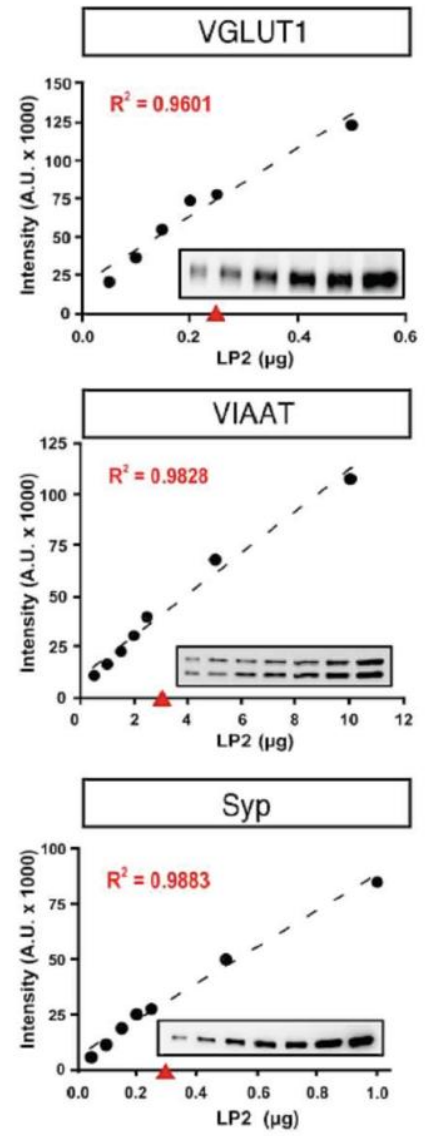
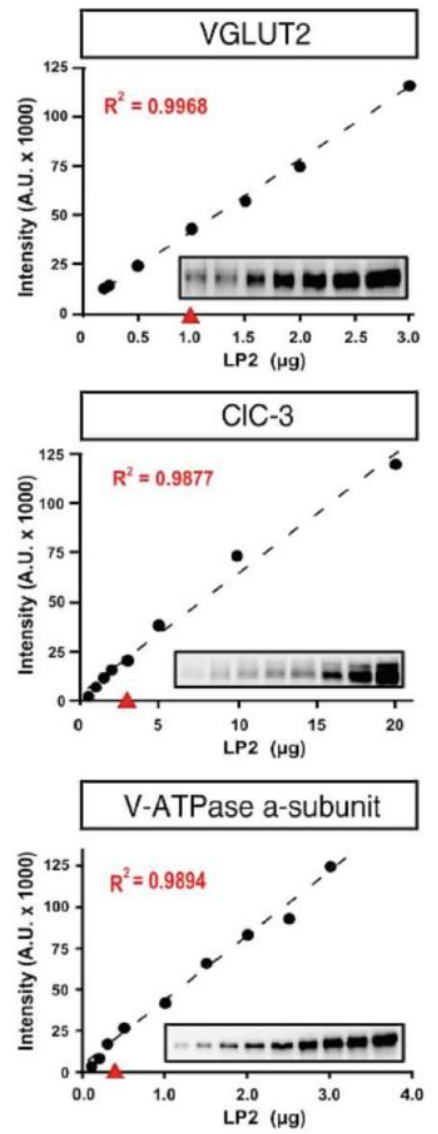

\subsubsection{Relevant protein levels in LP2-samples}

The results from the measurement of acidification in LP2 samples are supported by the quantitative analysis of relevant synaptic vesicle markers (Figs. 3.1e, 3.2e and 3.4a-d). This procedure ensured that the composition of the SV-samples is not altered concerning protein levels (see 2.2.1, 2.10.1 and 2.10.2 for details).

Since the quantification of protein levels with western blots critically depends on carefully calibrated antibodies and well adjusted amounts of samples being loaded on SDS-gels, a calibration curve was generated for each of the tested proteins. The procedure revealed that 
the proteins tested herein gave reliably linear responses within the range that was tested (Fig 3.5).

\subsection{Anion dependence of glutamate induced acidifcation in LP2 samples}

The glutamate-dependent acidifcation of SVs depends, analogous to the uptake, on low millimolar concentrations of extravesicular $\mathrm{Cl}^{-}$. The readout of this transport is the fluorescence quenching of $\mathrm{AO}$ due to luminal acidifcation. This clearly indicates that protons are the pair ion of glutamate in the lumen. The assay is however different from radiotracer experiments because it is not possible to clearly distinguish which of the two anions $\left(\mathrm{Cl}^{-}\right.$and/or glutamate) is quantitatvely contributing to the signal change. Higher concentrations of $\mathrm{Cl}^{-}$increase the signal and therefore overlap with the glutamate-induced acidification (in the measurement of glutamate uptake with radiotracers high $\mathrm{Cl}^{-}$ concentrations decrease the signal). Interestingly though, especially in the light of the data from KO-animals, which suggest a conductivity for anions in VGLUT1 itself (Figs. 3.2a-d and 3.3), the acidification in the presence of $10 \mathrm{mM}$ glutamate alone and of $4 \mathrm{mM}$ extravesicular $\mathrm{Cl}^{-}$do not sum up to the acidification caused by the two substances when added simultaneously. This fact has been long known, however it was not generally interpreted as the result of a $\mathrm{Cl}^{-}$conductance on SVs. If the activation is resulting from a barely measurable establishment of a $\Delta \mathrm{pH}$ due to a conductance for small anions in VGLUT1, which boosts subsequently a proton/glutamate exchange (that still might require $\Delta \Psi)$, then the assay can also reveal conductivities for other anions that enhance glutamate uptake, and potentially can also permeate VGLUT1.

Therefore it was tested if a similar correlation can be seen for $\mathrm{NO}_{3}{ }^{-}$a small monavalent anion that is conducted by many $\mathrm{Cl}^{-}$channels, including ClCs (Ryan and Mindell, 2007). $\mathrm{NO}_{3}{ }^{-}$does however inhibit the V-ATPase at high concentrations (Inoue et al., 2005; Muller et al., 1996) and thus the glutamate-based acidification assay could prove useful to see if SVs bear a conductivity for this anion. Indeed, $\mathrm{NO}_{3}{ }^{-}$does enhance glutamate induced acidifcation (Fig. 3.6c-d,g), gluconate however, a bulky anion, does not show such an effect (Fig 3.6e,g). This has been also previously reported, but was not interpreted as the result of a 
a
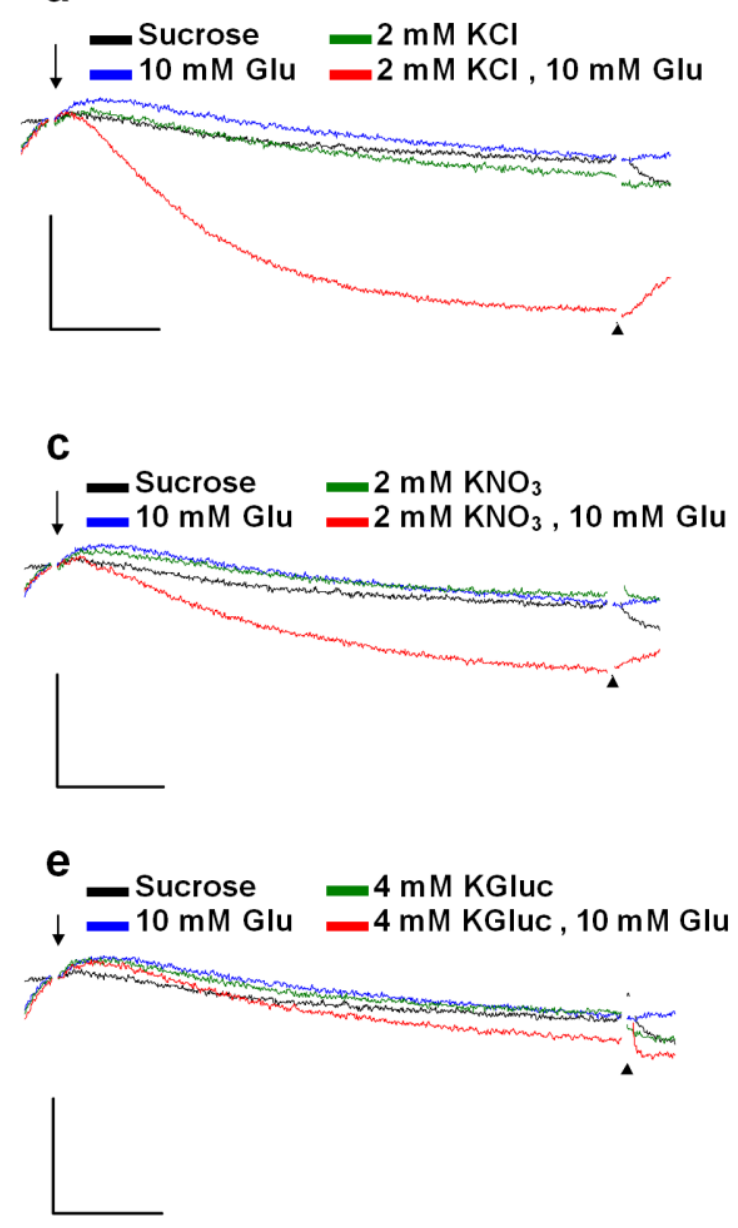

b
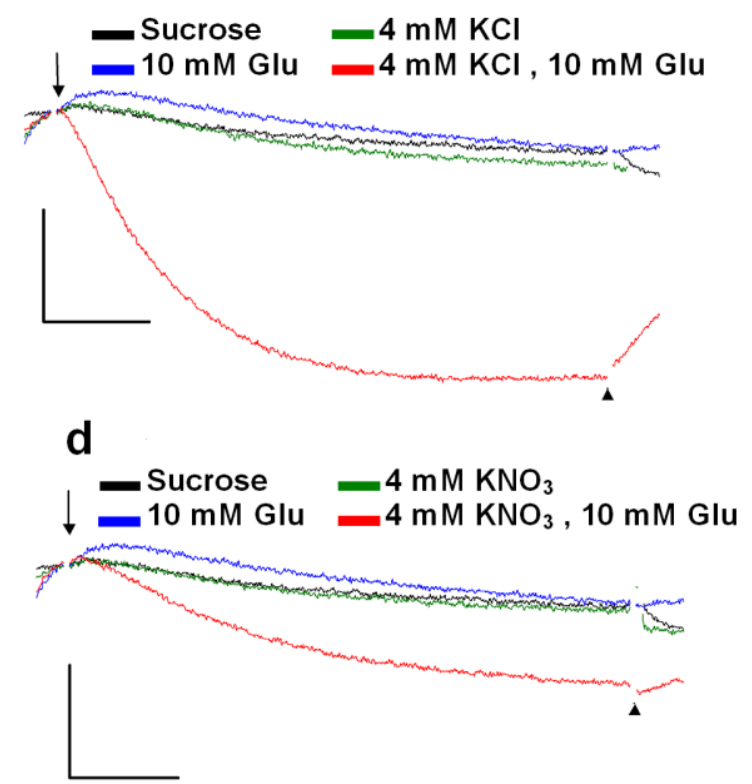

f

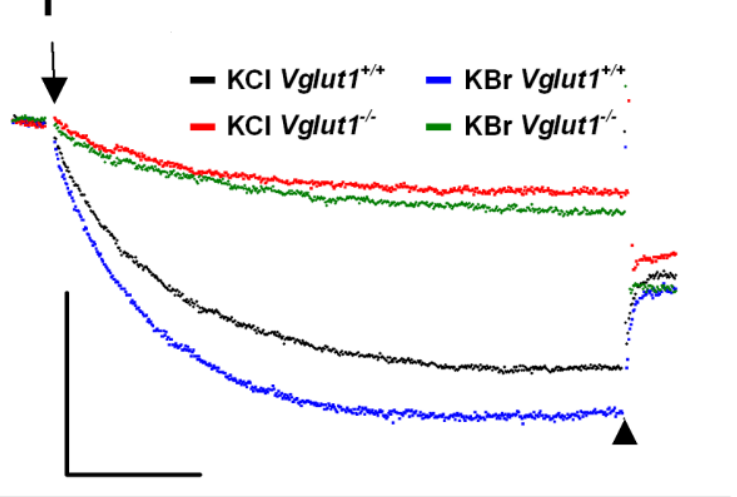

Figure 3.6

Anion dependence of glutamate induced acidification in LP2 samples

$(\mathbf{a}-\mathbf{e}, \mathbf{g})$ Different anions were tested for their ability to induce glutamate dependent acidification in SVs at concentrations of $2 \mathrm{mM}(\mathrm{a}, \mathrm{c})$ and $4 \mathrm{mM}(\mathrm{b}, \mathrm{d}, \mathrm{e}, \mathrm{g})$ compared to sucrose (black) or potassium glutamate alone $(10 \mathrm{mM}) . \mathrm{KCl}$ was used in $(\mathrm{a}-\mathrm{b}), \mathrm{KNO}_{3}$ in (c-d) and KGluc in (e). A quantitative representation is shown in (g). Note that $\mathrm{Cl}^{-}$and $\mathrm{NO}_{3}{ }^{-}$increase the signal when added together with glutamate but gluconate shows only a very small effect. (f) The conductance of $\mathrm{Br}^{-}(30 \mathrm{mM})$ was compared with $\mathrm{Cl}^{-}(30 \mathrm{mM})$ in LP2 samples from Vglut $1^{+/+}$and Vglut $^{-1 /}$ mice respectively. The data suggest that $\mathrm{Br}^{-}$also permeates VGLUT1. All traces are from single experiements. The scale bars represent $120 \mathrm{~s}$ and $500 \mathrm{AU}$. Addition of ATP and FCCP are indicated with an arrow and arrowhead respectively. 
conductivity (Moriyama and Yamamoto, 1995). The results were instead interpreted as an enhancement of the V-ATPase by anions, although $\mathrm{NO}_{3}{ }^{-}$is known to inhibit the $\mathrm{V}$ ATPase.

The conductivity for $\mathrm{Br}^{-}$(another halide that passes most $\mathrm{Cl}^{-}$channels was directly measured in the absence of glutamate for vesicles from WT and knock-out brains (Fig. 3.6f). The results suggest that $\mathrm{Br}^{-}$can also permeate VGLUT1 since the acidification is much greater when VGLUT1 is present on SVs. An enhancement of glutamate uptake has been reported also for low millimolar concentrations of $\mathrm{Br}^{-}$that is equally efficient as $\mathrm{Cl}^{-}$ (Moriyama and Yamamoto, 1995). The data shown in figure 3.6 indicates the permeation of $\mathrm{NO}_{3}{ }^{-}$through VGLUT1. $\mathrm{NO}_{3}{ }^{-}$can be useful in studies that involve collisional quenching of halide-sensitive dyes such as SPQ, MEQ or MQAE, that are not quenched by $\mathrm{NO}_{3}{ }^{-}$. These dyes elegantly demonstrated a $\mathrm{Cl}^{-}$permeation through bacterial homologues of plasma membrane glutamate transporters and involved the use of $\mathrm{NO}_{3}{ }^{-}$(Ryan and Mindell, 2007).

\subsection{Estimation of the copy number of $\mathrm{ClC}-3$ on synaptic vesicles}

ClC-3 is mostly referred to as a SV-resident (besides being localized to endosomal structures in other tissues than brain), since not only some functional data point towards this but, also because there appears to be a clear enrichment of this protein as judged by immunoblotting of purified SVs (Stobrawa et al., 2001) (Fig 3.7). However, based on the results from two $\mathrm{KO}$ lines described above, it would be also of importance to to test if $\mathrm{ClC}$ 3 is a genuine SV-resident.
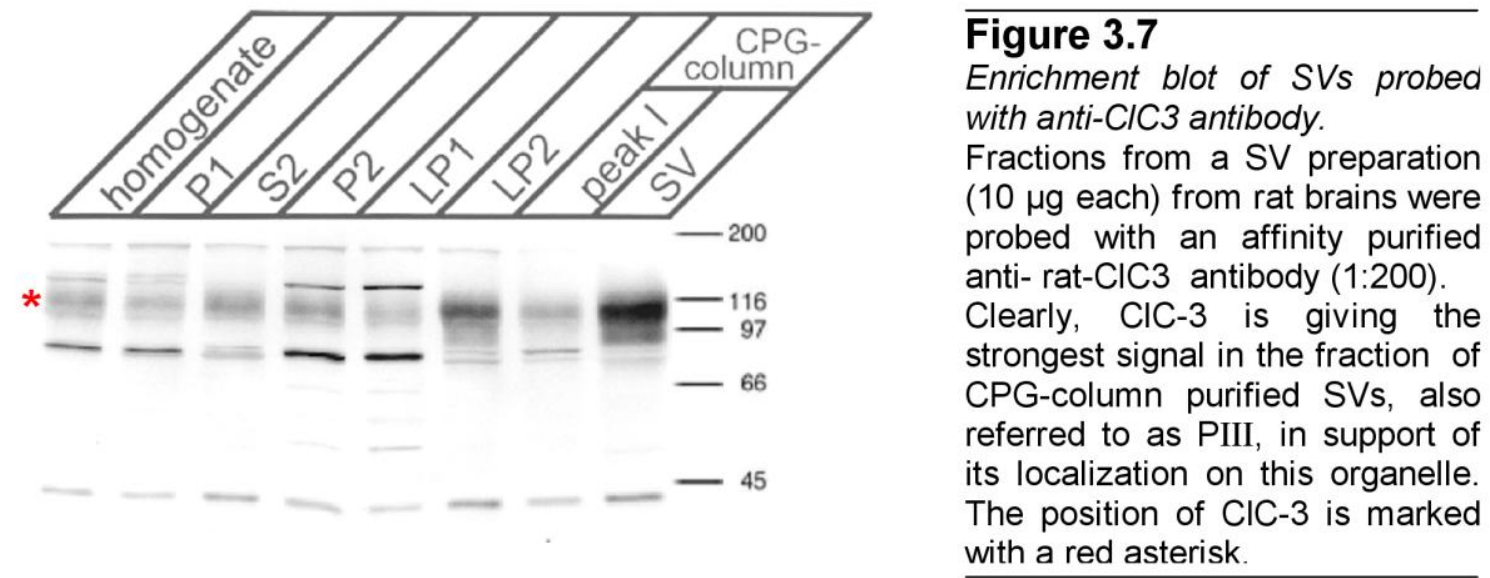

To estimate the copy number of ClC-3 on SVs, a comparison with the copy number of 
VGLUT1 was chosen. For this, ClC-3 and VGLUT1, both C-terminally tagged with GFP (see 2.10.3) (EGFP and Venus-YFP for ClC-3 and VGLUT1 respectively) were expressed in tsA201 cells (see 2.4). When lysates of cell lines expressing either GFP-tagged VGLUT1 or ClC-3 were probed with an anti-GFP serum (see 2.10.2) it was evident that the ClC-3 expression level was dramatically lower than for heterologous VGLUT1 protein. (Fig 3.8a). Both plasmids were transfected with similar efficiency and both yielded clearly fluorescent cells. The VGLUT1 protein was, conservatively estimated, expressed at 50fold higher level (Fig 3.8b) compared to $\mathrm{ClC}-3$.

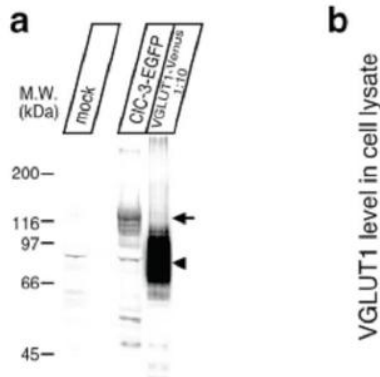

WB: anti-GFP Ab
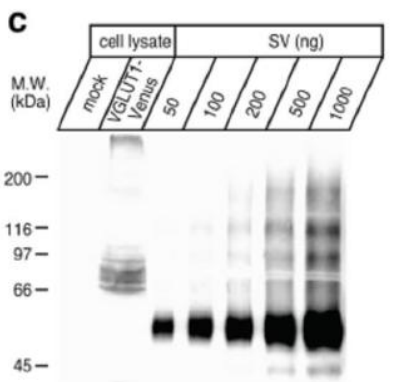

WB: anti-VGLUT1 Ab

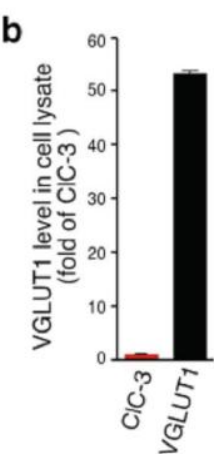

Figure 3.8

Estimation of the CIC-3 copy number on SVs.

(a) Comparison of cell lysates expressing CIC-3-EGFP and VGLUT1-Venus (1:10 dil.) with an anti-GFP serum. VGLUT1 fusion protein is marked with an arrowhead and $\mathrm{ClC}-3$ with an arrow. (b) Quantitative representation of results from triplicates of (a) corrected for dilution. (c and (d) Signal comparison of the cell lysates (probed in (a) and (b)) with increasing amounts of rat CPG-SVs using specific antibodies for either VGLUT1 (c) and CIC3 (d). Represantitive blots are shown (e) Quantitative evaluation of the signal of CIC-3 and VGLUT1 in heterologous lysates compared to SVs based on immunoblots seen in (c) and (d). See text for details.

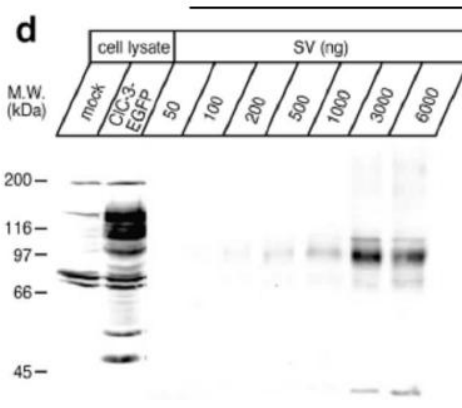

WB: anti-CIC-3 Ab

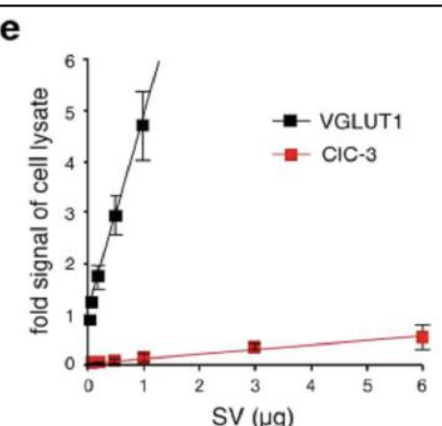

Then cell lysates expressing ClC-3-EGFP were probed along with purified SVs from rat brains (Fig. 3.8c-d) with an anti-ClC-3 antibody. The lysates of VGLUT1-expressing cells were also compared with SVs. For this, the two standards (cell lysates) were compared with different amounts of SVs from rat brain by using specific antibodies as shown in figure $3.8 \mathrm{e}$ from triplicate measurements. While the standard for VGLUT1 roughly corresponds to $100 \mathrm{ng}$ of SVs, the signal of the standard in the case of $\mathrm{ClC}-3$ exceeds even $6 \mu \mathrm{g}$ of SVs. Therefore the amount of ClC-3- and VGLUT1-standards compared to SVs differs roughly estimated by a factor of more than 100. Given that the standard for VGLUT1 contains $~ 50$ times more VGLUT1-molecules than the standard for ClC-3, the 
actual copy number of $\mathrm{ClC}-3$ on $\mathrm{SVs}$ is about 5000 times lower than the copy number of VGLUT1.

Since the copy number of VGLUT1 has been measured together with a dozen of other SVresidents in a relatively precise manner (Takamori et al., 2006) ( 10 copies / VGLUT1type positive SV), one can deduce that less than every $2000^{\text {th }} \mathrm{SV}$ may bear a ClC-3 dimer (the functional oligomeric state). Since this difference is dramatic, ClC-3 is likely only a passenger on SVs or a contaminant from endosomal structures which have co-purified with SVs. These results further support the data in 3.1.

\subsection{Purification of proton pumps, VGLUT1 and liposome formation}

\subsubsection{Purification of proton pumps}

In order to verifiy the $\mathrm{Cl}^{-}$conductance of VGLUT1 with purified components and investigate the influence of controlled ionic environments on glutamate translocation, it is necessary to energize the artficial membranes. This could be achieved with chemical gradients and certain ionophores such as valinomycin $\left(\mathrm{K}^{+}\right.$-ionophore $)$Nigericin $\left(\mathrm{K}^{+} / \mathrm{H}^{+}\right.$exchanger) or FCCP ( $\mathrm{H}^{+}$-ionophore) (Hell et al., 1991; Shioi and Ueda, 1990). However, such gradients are not very long lasting. An ion pump which would be co-reconstituted together with the transporter would give a better control and broader application for such experiments. This pump would need to be electrogenic, largely independent of the ionic composition, relatively easy to obtain and biochemically stable enough for storage and active under a range of test conditions. Vesicular glutamate transport has been driven already with exogeneous pumps like bacteriorhodopsin (Maycox et al., 1990a) and the $E$. coli ATP-Synthase (Moriyama et al., 1991). While bacteriorhodopsin is relatively easy to purify and the reconstitution is well established, it becomes technically problematic to use in combination with fluorescent dyes (activation by light).

The properties of the native V-ATPase from SVs are certainly very well suited for the purpose which has been demonstrated with a V-ATPase purified from bovine clathrin coated vesicles (Xie et al., 1989). However, the purification of this low-copy number enzyme from SVs requires very high amounts of SVs. Therefore the ATP-Synthase was 
chosen since it is abundant (in mitochondria or bacteria), thus amenable to successful purification.

a
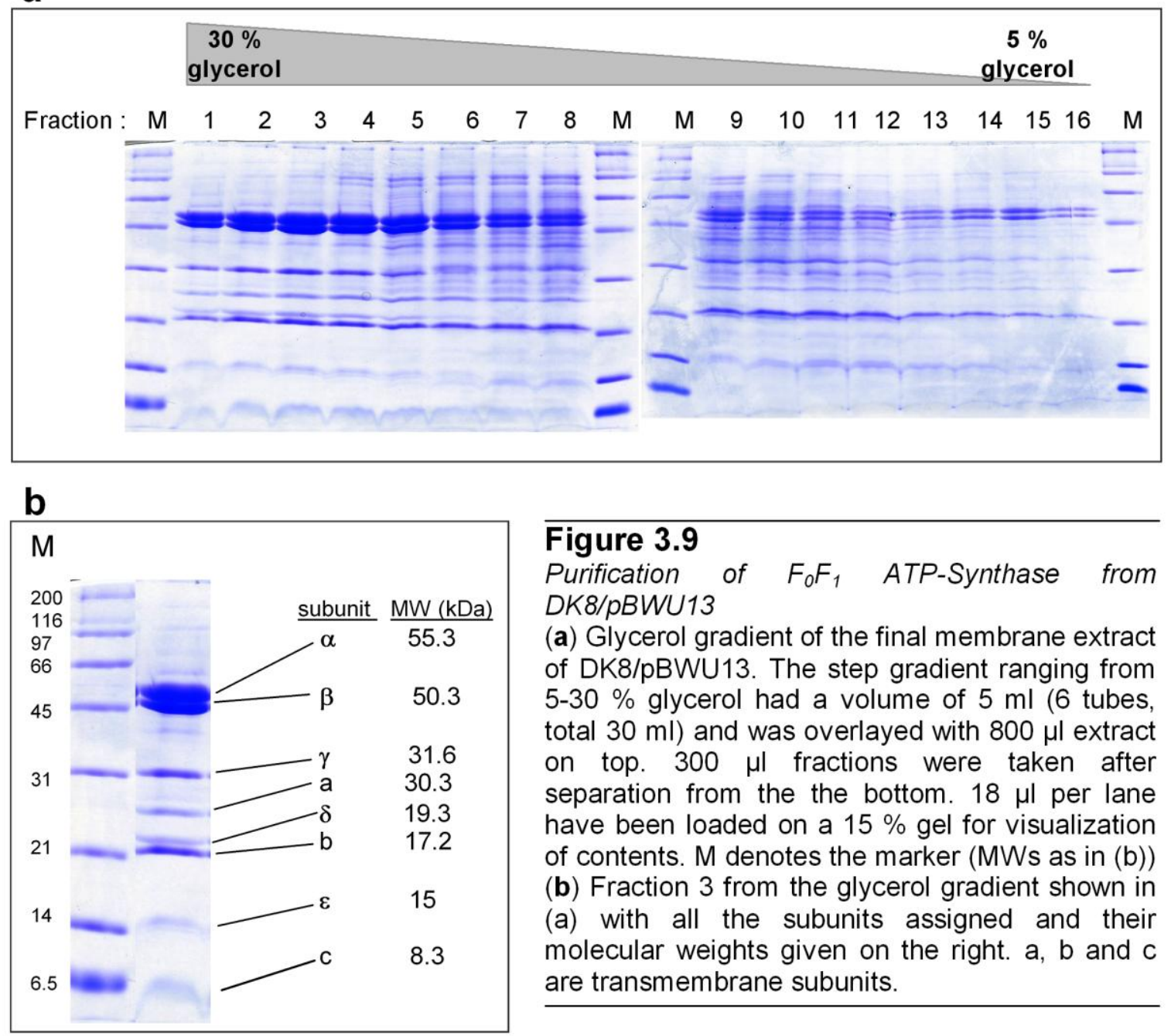

Figure 3.9

Purification of $F_{0} F_{1}$ ATP-Synthase from DK8/pBWU13

(a) Glycerol gradient of the final membrane extract of $\mathrm{DK} 8 / \mathrm{pBWU} 13$. The step gradient ranging from $5-30 \%$ glycerol had a volume of $5 \mathrm{ml}$ (6 tubes, total $30 \mathrm{ml}$ ) and was overlayed with $800 \mu$ l extract on top. $300 \mu \mathrm{l}$ fractions were taken after separation from the the bottom. $18 \mu \mathrm{l}$ per lane have been loaded on a $15 \%$ gel for visualization of contents. M denotes the marker (MWs as in (b)) (b) Fraction 3 from the glycerol gradient shown in (a) with all the subunits assigned and their molecular weights given on the right. $a, b$ and $c$ are transmembrane subunits.

\subsubsection{Purification of $\mathrm{F}_{0} \mathrm{~F}_{1}$ wild type ATP-synthase of $E$. coli}

The native E.coli ATP-Synthase, was constitutively overexpressed in DK8-cells (native $u n c$-operon deleted) on a minimal medium following an established protocol (see 2.3.1 for a detailed description). The final step of this purification procedure involved a glycerol gradient (Fig 3.9a). The $\mathrm{F}_{0} \mathrm{~F}_{1}$ complex migrated into the bottom fractions of this gradient with a mass of $\sim 500 \mathrm{kDa}$. All subunits could be identified in Coomassie Blue stained SDSgels (Fig 3.9b). The fractions containing $\mathrm{F}_{0} \mathrm{~F}_{1}$ complex of good purity were pooled, aliquoted and stored at $-80^{\circ} \mathrm{C}$ until use. 


\subsubsection{Purification of recombinant His-tagged ATP-synthase from the thermophilic Bacillus sp. PS3 $\left(\mathrm{TF}_{0} \mathrm{~F}_{1}\right)$}

Since the wild type $\mathrm{F}_{0} \mathrm{~F} 1$ proved problematic in the AO-acidification assay (see 3.4.3), presumably due to impurities, a purification that involes an affinity tag was attempted.

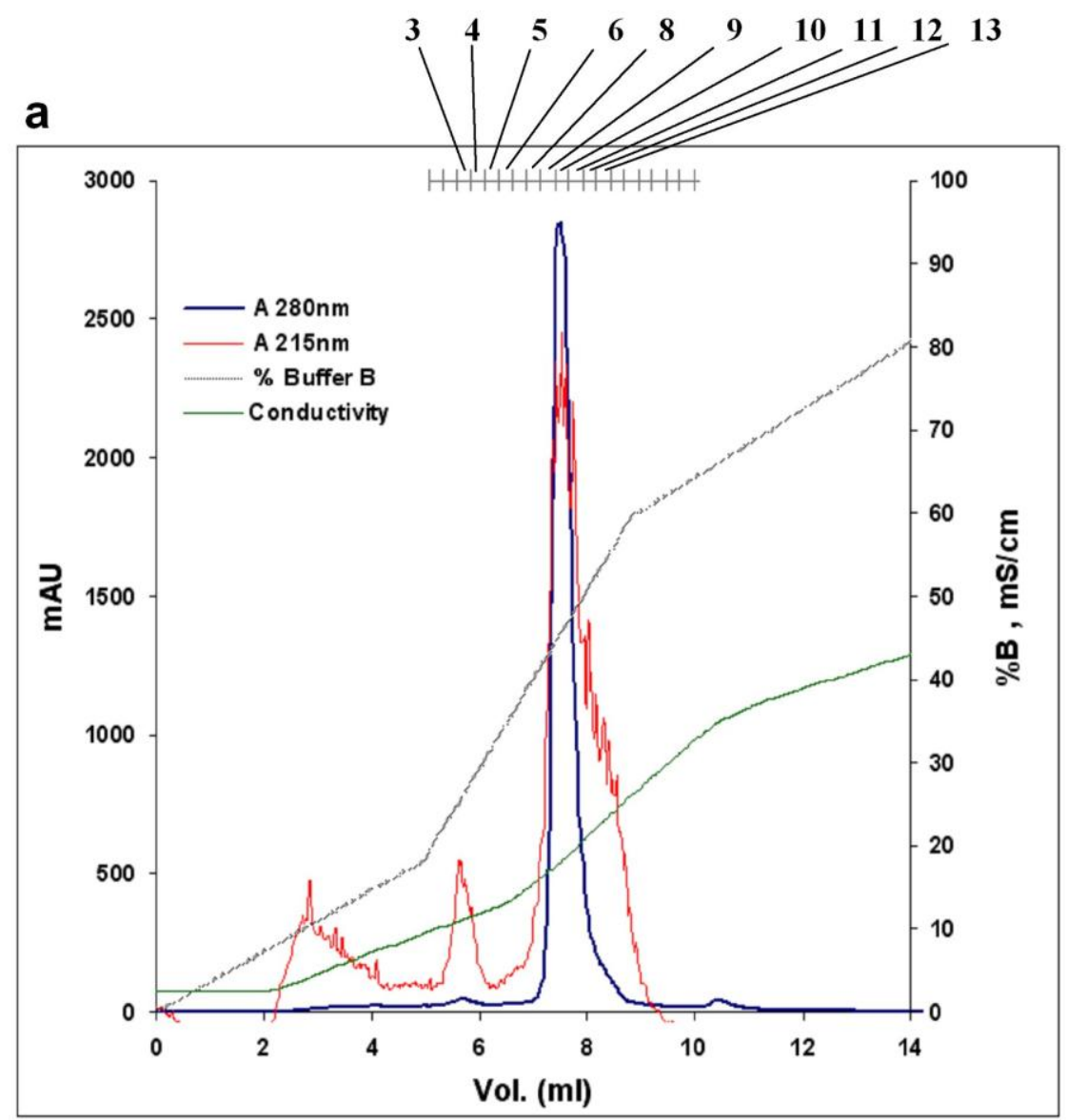

Figure 3.10

b

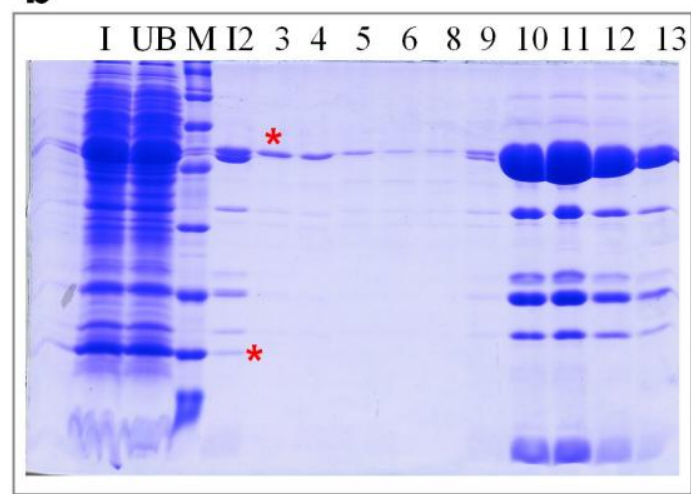

Anion exchange chromatography of $\mathrm{TF}_{0} \mathrm{~F}_{1}$

(a) Elution profile of $\mathrm{TF}_{0} \mathrm{~F}_{1}$ after $\mathrm{Co}^{2+}$-Affinity purification from a MonoQ $5 / 50$ column. The collected fractions are marked on top of the panel. $10 \mathrm{ml}$ Eluate could be efficiently concentrated to less than $1 \mathrm{ml}$ without disruption of the complex. See 2.3.2 for details. (b) Coomassie staining of a $15 \%$ SDS-gel of the fractions denoted above. The input material is marked as 12 . I and UB show input and unbound material of the $\mathrm{Co}^{2+}$ affinity chromatography. Some minor contaminants have been removed $(\sim 14 \mathrm{kDa})$ as well as single $\alpha$-subunits (in fractions $3-6$ ), both highlighted with a red asterisk. Equal volumes were loaded. 
Recently, the successful purification of a functional thermophilic ATP-Synthase has been reported (Suzuki et al., 2002). Therefore a purification of this protein was attempted.

The ATP-Synthase from the thermophilic Bacillus strain PS3 $\left(\mathrm{TF}_{0} \mathrm{~F}_{1}\right)$ was heterologously

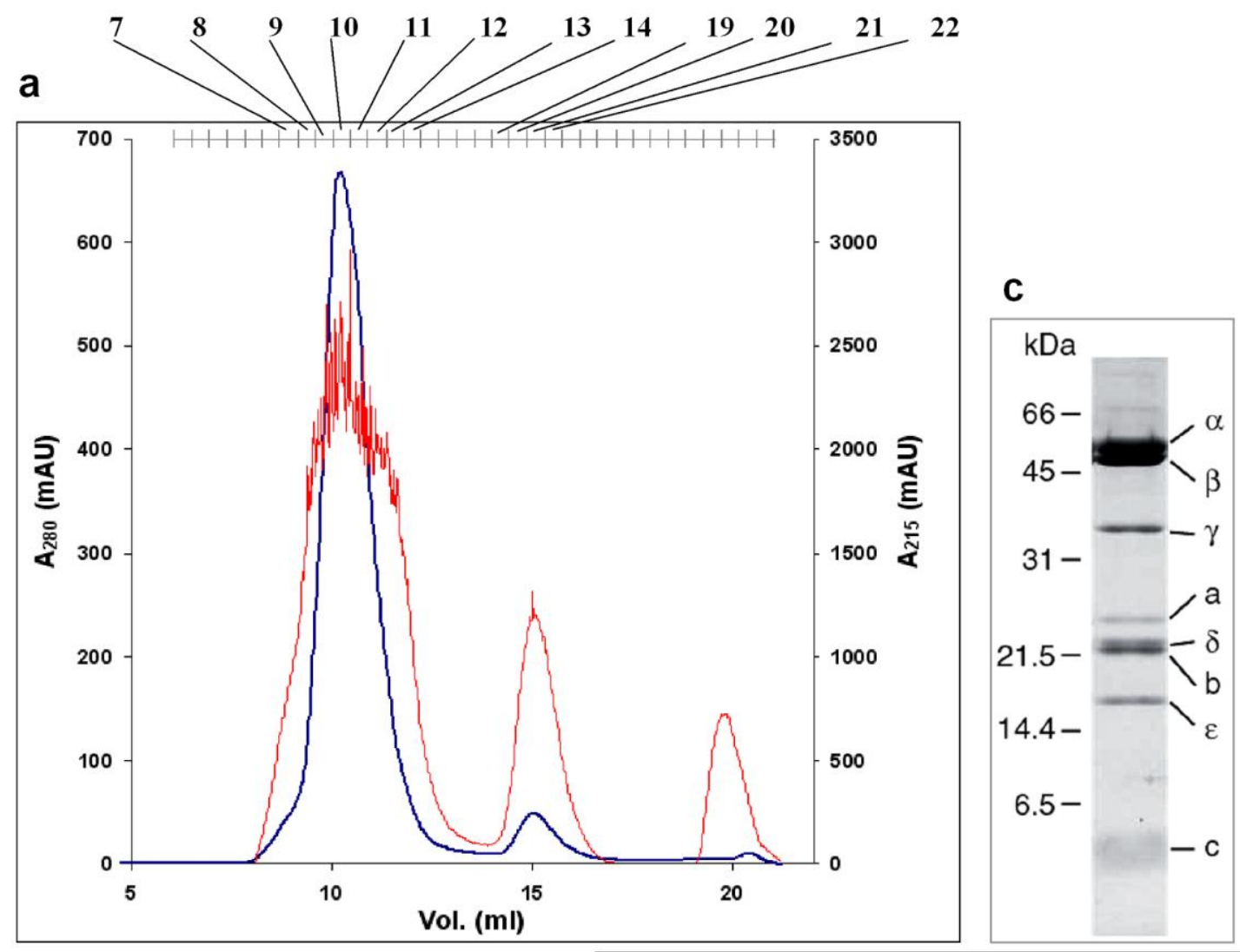

Figure 3.11

b

(a) Elution profile of $\mathrm{TF}_{0} \mathrm{~F}_{1}$ from a Superdex 200 M $78 \quad 9101112131419202122 \mathrm{M}$

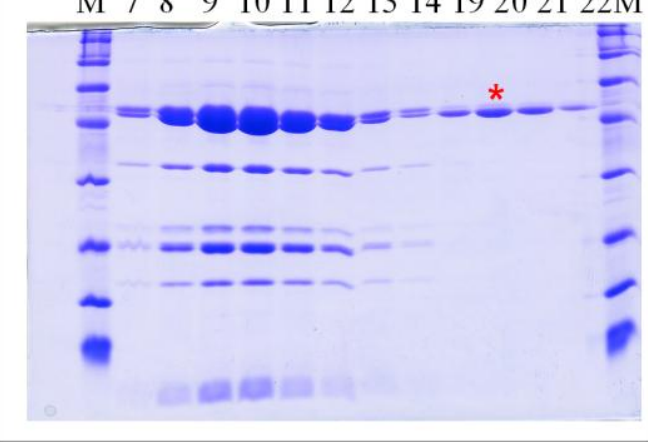

$10 / 30$ column. The collected fractions are marked on top of the panel. The absorption at 280 and $215 \mathrm{~nm}$ are shown in blue and red respectively. See 2.3.2 for details. (b) Coomassie staining of a $15 \%$ SDS-gel of the fractions denoted in (a). Unbound $\beta$-subunits (in fractions 19-22, red asterisk) migrate at lower molecular weight and were well separated from the holoenzyme (fractions 8-12). Equal volumes were loaded. (c) Coomassie stain of pooled fractions $\mathrm{TF}_{0} \mathrm{~F}_{1}$ after the final purification step with all subunits assigned. About $10 \mu \mathrm{g}$ of protein were loaded).

expressed in DK8 E. coli cells from the plasmid pTR19-ASDS (see 2.3.2 for a detailed description). It could be easily purified in a first step by the Hexa-His-tag at the b-subunit with $\mathrm{Co}^{2+}$ affinity beads and was further concentrated and purified on an anion-exchange column and finally eluted from a gel-filtration column. 
DDM was used as a solubilizing agent, and all subunits of the holoenzyme could be clearly identified (Fig. 3.11c). The non-ionic nature of DDM made the subsequent step of anion exchange chromatography straightforward. Some minor contaminants could be removed with anion exchange chromatography (Fig. 3.10), however this step served mainly to concentrate the eluate from the $\mathrm{Co}^{2+}$ column. A last purification step (SEC) rendered the sample very homogeneous. Only some unbound b-subunits could be seen migrating at lower molecular weight (Fig 3.11b). The stability upon storage was greatly improved by the size exclusion chromatography compared to the eluate from the $\mathrm{Co}^{2+}$-column. $\mathrm{TF}_{0} \mathrm{~F}_{1}$ lost its activity when it was frozen. Prevention of ice-formation with high glycerol concentrations made storage at $-20^{\circ} \mathrm{C}$ possible, but due to the high stability of the complex, storage at $4^{\circ} \mathrm{C}$ was sufficient to ensure activity for up to several months.

a

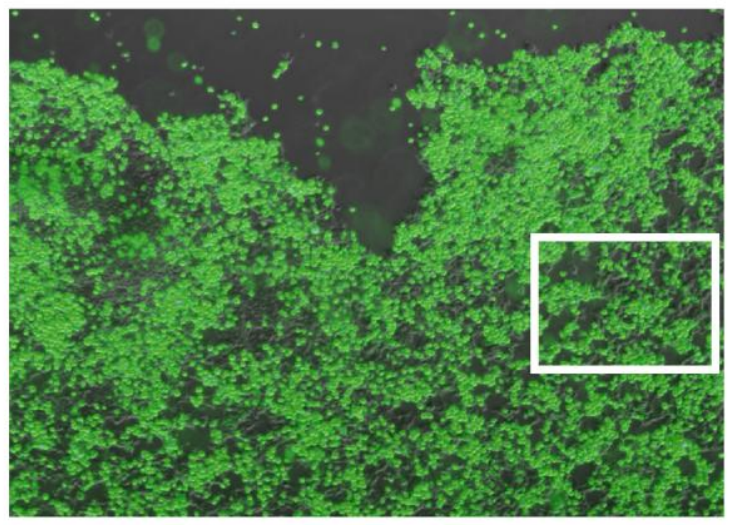

b

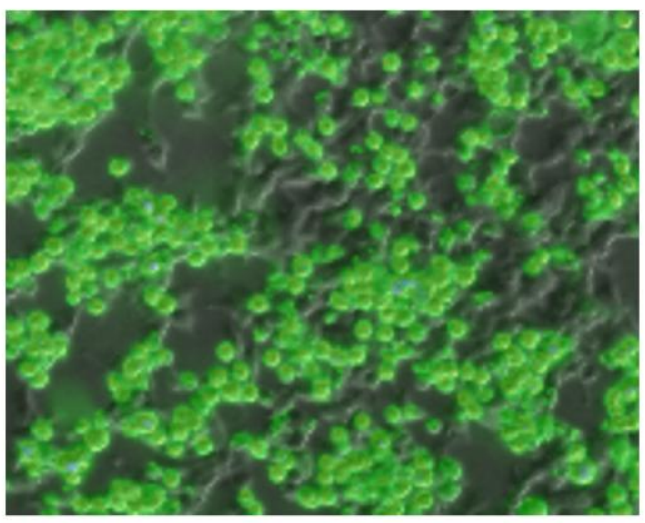

Figure 3.12

Expression of VGLUT1 in tsA201 cells using transient transfection with $\mathrm{Ca}_{3}\left(\mathrm{PO}_{4}\right)_{2}$ under control of the CMV promoter

(a) Typical transfection of tsA201 cells with VGLUT1-Venus (merged image of DIC and GFP-fluorescence) (b) Enlarged area highlighted in (a). Note the high percentage of cells producing VGLUT1-Venus protein. VGLUT1 expressing cells often round up and detach after $\sim 30 \mathrm{hrs}$. The image was taken $\sim 20 \mathrm{hrs}$ after the transfection.

\subsubsection{Heterologous expression and purification of VGLUT1 in tsA201 cells}

The results demonstrated in the chapter 3.1 - 3.12 clearly indicated an anion conductance in VGLUT1. To investigate the function of this conductance it would be essential to measure the uptake of glutamate in a controlled environment, ideally in artificial vesicles which makes it possible to define the ionic composition of the interior and exterior of vesicles. In addition, reconstituted and purified VGLUT1 would be necessary to verifiy 
that the $\mathrm{Cl}^{-}$conductance is indeed an intrinsic property of the transporter, away from its native membrane to rule out the influence of a different, unknown factor.

The human cell line tsA201, a derivative of the immortal HEK293 (human embryonic kidney) cells is one of the most suitable mammalian cell lines for the overexpression of plasmid-encoded genes. It is stably transformed with the temperature sensitive $\mathrm{T}$ -
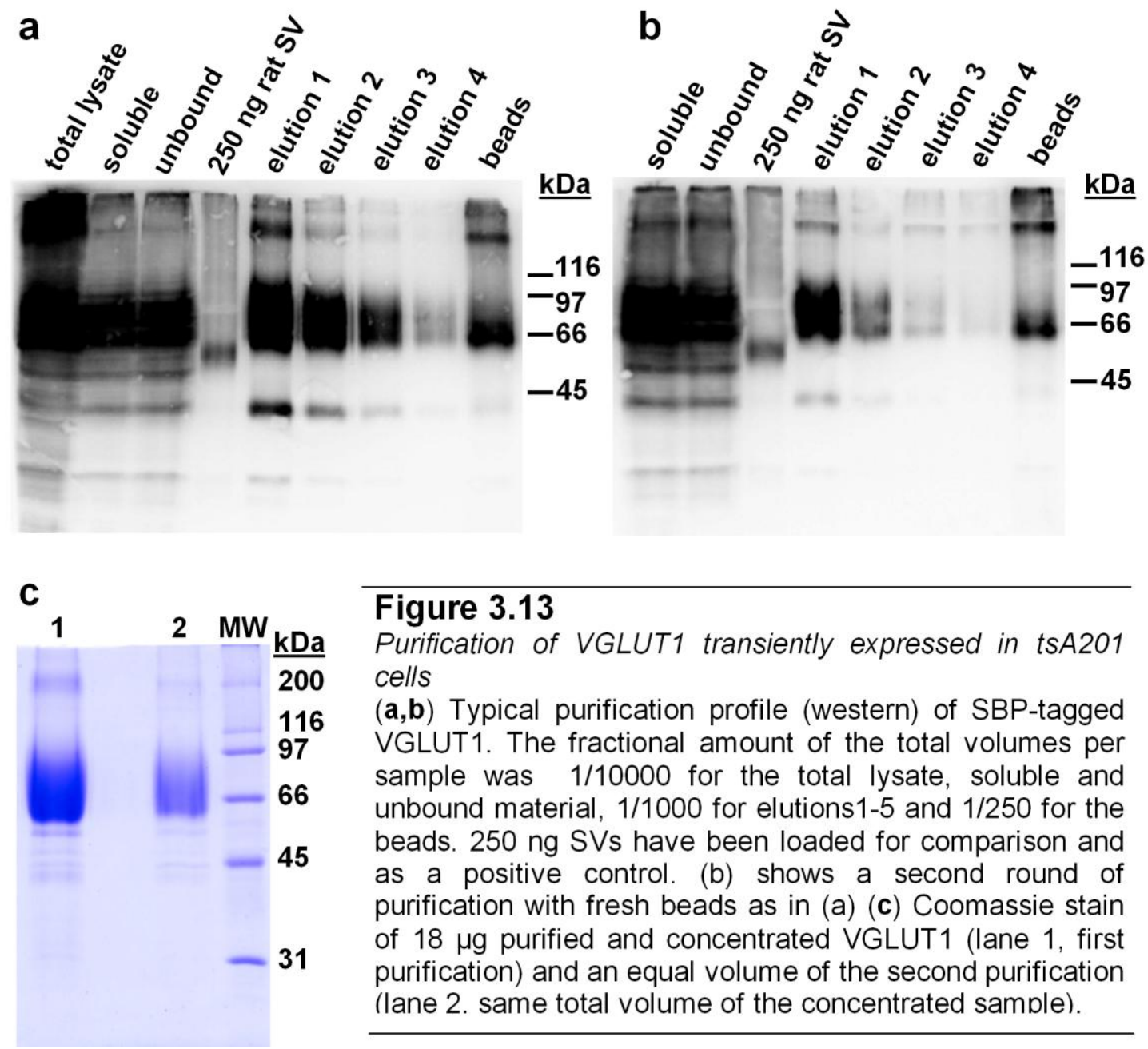

Figure 3.13
Purification of VGLUT1 transiently expressed in tsA201
cells
(a,b) Typical purification profile (western) of SBP-tagged
VGLUT1. The fractional amount of the total volumes per
sample was $1 / 10000$ for the total lysate, soluble and
unbound material, $1 / 1000$ for elutions $1-5$ and $1 / 250$ for the
beads. $250 \mathrm{ng}$ SVs have been loaded for comparison and
as a positive control. (b) shows a second round of
purification with fresh beads as in (a) (c) Coomassie stain
of $18 \mu$ purified and concentrated VGLUT1 (lane 1 , first
purification) and an equal volume of the second purification
(lane 2. same total volume of the concentrated samble).

antigen from the simian vacuolating virus 40 (SV40). If a transfected plasmid contains an SV40 origin of replication (ori) several copies of the plasmid may persist in a single transfected cell even after mitotic division. This results in high expression levels of recombinant proteins when used in combination with a powerful promoter such as the CMV promoter from the cytomegalo virus.

Since it is not feasible to express polytopic eukaryotic membrane proteins in bacteria only a limited number of approaches is left. Typically a natural source would be chosen or 
heterologous expression in insect cells (Sf9, Schneider cells) or mammalian cell lines (e.g. CHO, HEK293). Due to the simple and inexpensive transfection procedure and high transfection efficiency as well as the easy cultivation, the tsA201 cell line was chosen for a transient expression approach (Fig. 3.12).

A key feature of the herein described purification scheme is the use of a genetically encoded high-affinity tag for a fast and non-denaturing purification. While a classical oligo-histidine (6-10 His) tag would also allow for non-denaturing isolation of a target protein it has been virtually inefficient (not shown) due to the low expression achieved for a large membrane protein like VGLUT1. Hence, a high affinity interaction, was needed to purify VGLUT1 from cell lysates.

A genetically encoded and reasonably small (31 amino acids) fully synthetic sequence that tightly binds to streptavidin was found in an mRNA-display approach (Wilson et al., 2001). The authors of this study succeeded in finding a sequence that binds with nanomolar affinity to native streptavidin, different from the well established Strep-TagII (IBA, Göttingen) which requires a mutant streptavidin. Since the native streptavidin retains its binding for biotin, which the Strep-TagII-system does not, the binding peptide could be selected for very high binding strength, yet still being releasable from streptavidin with (+)-biotin. Streptavidin is not sensitive to high concentrations of DDM (however binding was abolished by cholate) and elution is quick and highly specific which improves the purity of the isolated protein. In figure 3.13a a typical purification profile can be seen. A significant amount of the total VGLUT1-protein could be solubilized (50-70\%) from the cell membranes. Of the solubilized protein about $50 \%$ was captured by the beads. A second incubation of the unbound material with fresh beads did somewhat improve the yield (Fig. 3.13b). The protein eluted easily from the column after application of (+)-biotin. Only minor amounts of probably aggregated VGLUT1 remained on the beads (note that the fractional amount of the beads loaded in figure 3.13a-b is much higher than for the eluates). Another notion is that VGLUT1 generally migrates at higher molecular weight when expressed in tsA201/HEK293 cell lines compared to the native protein from brain. This is very likely due to a different and less defined glycosylation. Another indication for this is visible in the size exclusion profile in figure 3.21. To which extent this glycosylation influences the function is not known. The eluates were pooled and concentrated $\sim 20$-fold with spin concentrators to $\sim 1 \mathrm{mg} / \mathrm{ml}$ VGLUT1. This led to relatively high concentrations 
of DDM in the concentrate $(\sim 1 \%)$ since DDM, with a micellar molecuar weight of 50 $\mathrm{kDa}$, is also largely retained on the cis-side of the concentrator membrane (50 kDa cut-off). The purified and concentrated transporter (Fig. 3.13c) showed no major contaminations, very little aggregates (see Fig 3.21) and minor breakdown products. With a yield of $2-4 \mu \mathrm{g}$ per dish a reliable source was established to perform several series of experiments yet still requiring a very economical experimental layout.

Recently, the successful expression of mutant VGLUT2 proteins (based on a computergenerated model of VGLUT2) in insect cells (Baculovirus-Sf-9 expression) that greatly abolish glutamate transport, has been reported (Juge et al., 2006). These mutants could prove useful in the evaluation of the potential $\mathrm{Cl}^{-}$in VGLUT1. However, when the corresponding mutations were introduced into VGLUT1 (H120A and R176E, see 2.10.3) the expression level was much lower than for the wild-type protein (Fig 3.14a). Nevertheless

it

was

tried a

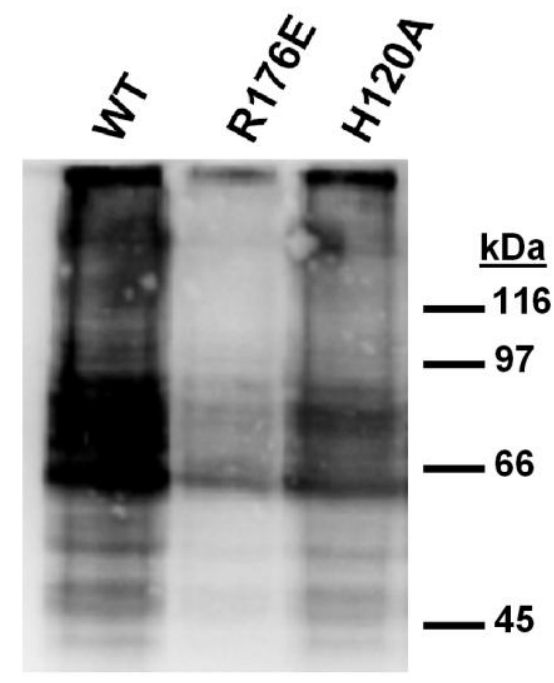

b

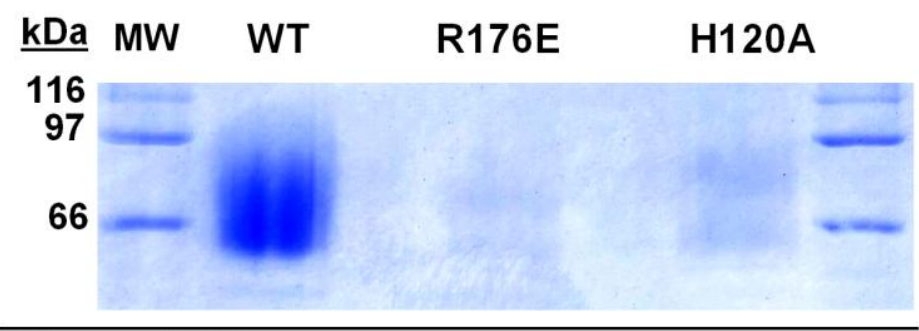

Figure 3.14

Purification of the VGLUT1 mutant proteins R176E and $\mathrm{H} 120 \mathrm{~A}$

(a) Soluble supernatants of WT and two mutant proteins of VGLUT1 expressed in tsA201 cells and probed with an anti-VGLUT1 antibody. (b) Coomassie stain of the purified proteins (all concentrated to the same volume with equal volumes loaded). Note the very low expression level of these mutants

purify the mutant transporters on a small scale starting from $3010 \mathrm{~cm}$-dishes in parallel with wild type VGLUT1. The direct comparison of concentrated samples with wild type protein in a Coomassie stained gel confirmed much lower expression levels. Due to the low amounts of the purified mutant proteins H120A and R176E (Fig 3.14b) in this small scale screen, further trials were not conducted. 


\subsubsection{Formation of liposomes and comparison of the $F_{0} F_{1}$ and $T F_{0} F_{1} A T P$ -}

\section{synthases}

Before VGLUT1 was co-reconstituted with a proton pump, the properties of the two different ATP-Synthases and the artificial bilayer were tested. Furthermore, since the transporter is solubilzed with DDM, the reconstitution required special considerations because the removal of this long-chain detergent from lipid-protein micelles demands other techniques than the widely used dialysis, dilution or gel-filtration.

To tackle these questions and find a procedure that allows to clearly reveal an anionconductance, either $\mathrm{F}_{0} \mathrm{~F}_{1}$ or $\mathrm{TF}_{0} \mathrm{~F}_{1}$ were mixed with solubilized phospholipids and solubilized cholesterol in small volumes of 500-2000 $\mu 1$ with a lipid-concentration

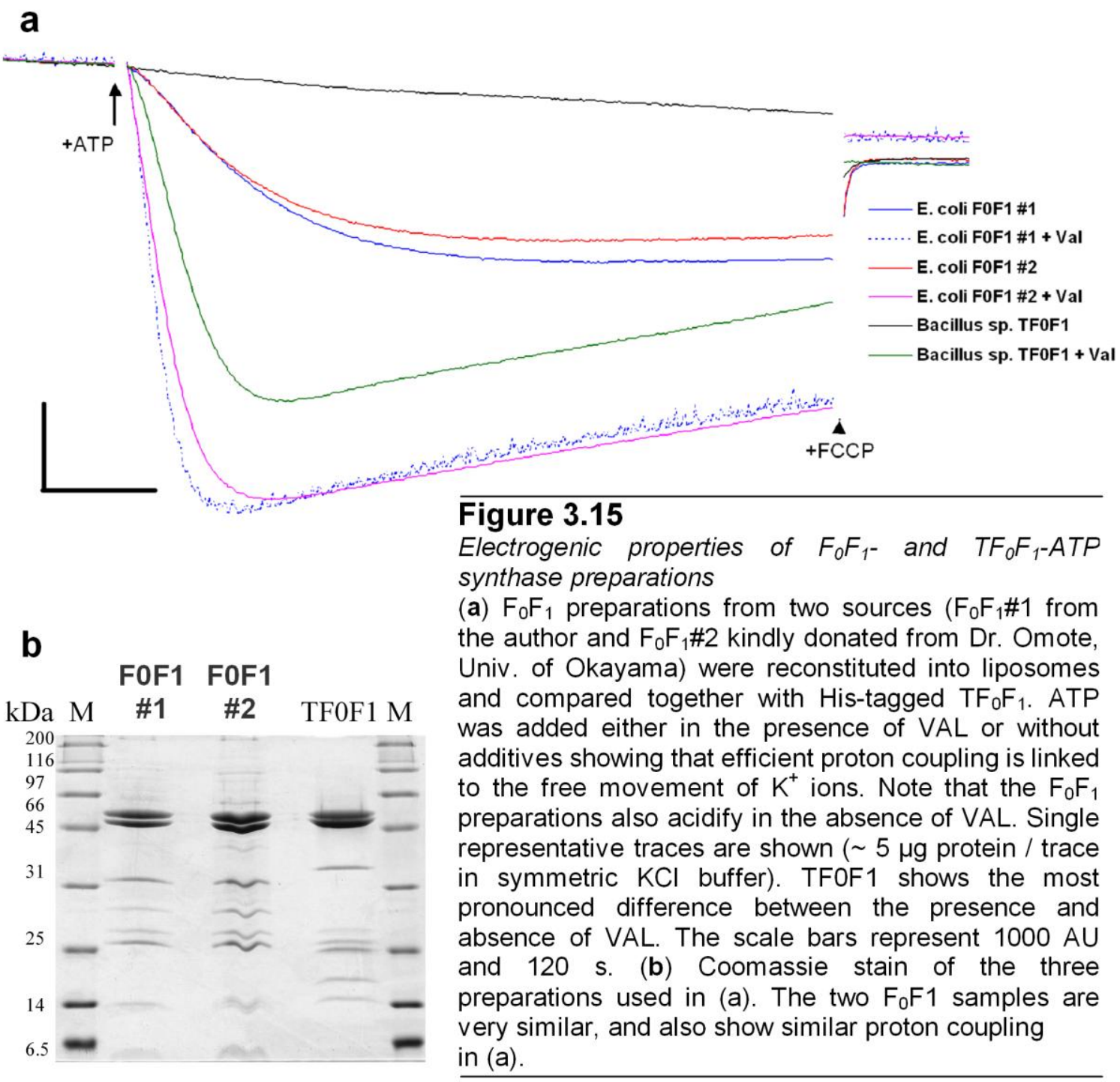


of $2.5 \mathrm{mg} / \mathrm{ml}$ for reconstitution (Fig. 3.15). The amount of DDM introduced by adding $\mathrm{TF}_{0} \mathrm{~F}_{1}$ was far below the critical micellar concentration (CMC) and thus unlikely to interfere with the measurement. $\mathrm{F}_{0} \mathrm{~F}_{1}$ from $E$. coli was also solubilzed in octyl-glucoside and thus well compatible with the lipid-mixture. The detergent was then removed by dialysis against $\mathrm{KCl}$-containing buffer (or other potassium salts) which led to liposome formation within few hours as judged by the turbid appearance of the sample (see 2.5 for details). Then, the samples were tested by assaying the AO quenching in symmetrical potassium based buffers in the presence of ATP. The use of potassium on either side (at least on the luminal side) of the membrane makes it possible to introduce a conductivity for this ion by adding valinomycin and thus allow for counterion movement, which is mandatory for the luminal acidifcation in the absence of an anion shunt (Xie et al., 1989). If the properties of the ATP-synthase-proteoliposomes are electrogenic, little or no proton movement should be seen in the absence of valinomycin, while it should be maximal upon addition of the ionophore. The difference in the acidifcation rates was then assessed to judge the suitabilty of the preparation for subsequent applications such as glutamate transport or testing $\mathrm{Cl}^{-}$permeation through VGLUT1.

Two $\mathrm{F}_{0} \mathrm{~F}_{1}$ preparations were tested, one from the author (\#2, Fig 3.15b, see 2.3.1 for details) and one from Dr. H. Omote (University of Okayama) (\#1, Fig 3.15b) who purified the protein-complex as described in the original protocol (Moriyama et al., 1991). The two $\mathrm{F}_{0} \mathrm{~F}_{1}$-preparations from E.coli show also relatively pronounced acidification in the absence of valinomycin, albeit it was strongly enhanced when valinomycin was added (Fig 3.15a) and are almost indistinguishable regarding their electrogenic properties as well as purity (Fig 3.15a-b). The conductivity underlying the acidification in the absence of valinomycin could not be identified. However, it was rather unselective since the effect could also observed by exchanging potassium with choline and also by exchanging $\mathrm{Cl}^{-}$with acetate or gluconate. Therefore porins come to mind because multiple forms of these large betabarrel transmembrane proteins with different selectivities appear in the outer membrane of gram-negative bacteria. Such porins, can be co-purified with proteins overexpressed and can interfere with the measurement of ion movements already in very small amounts due to their giant-conductances as reported for single channels recordings of the $\mathrm{Cl}^{-} / \mathrm{H}^{+}$ transporter ClCec1 (Accardi et al., 2004). 
The $\mathrm{F}_{0} \mathrm{~F}_{1}$ ATPase preparations were therefore not well suited for further investigations regarding VGLUT1. Albeit an additive $\mathrm{Cl}^{-}$conductivity should be visible as an additive signal change, the results would be less clear than for a preparation that show almost no acidification. Additionally, in uptake experiments any secondary effects from unknown ion movements would make the interpretations of the results more complicated and less trustworthy.

a
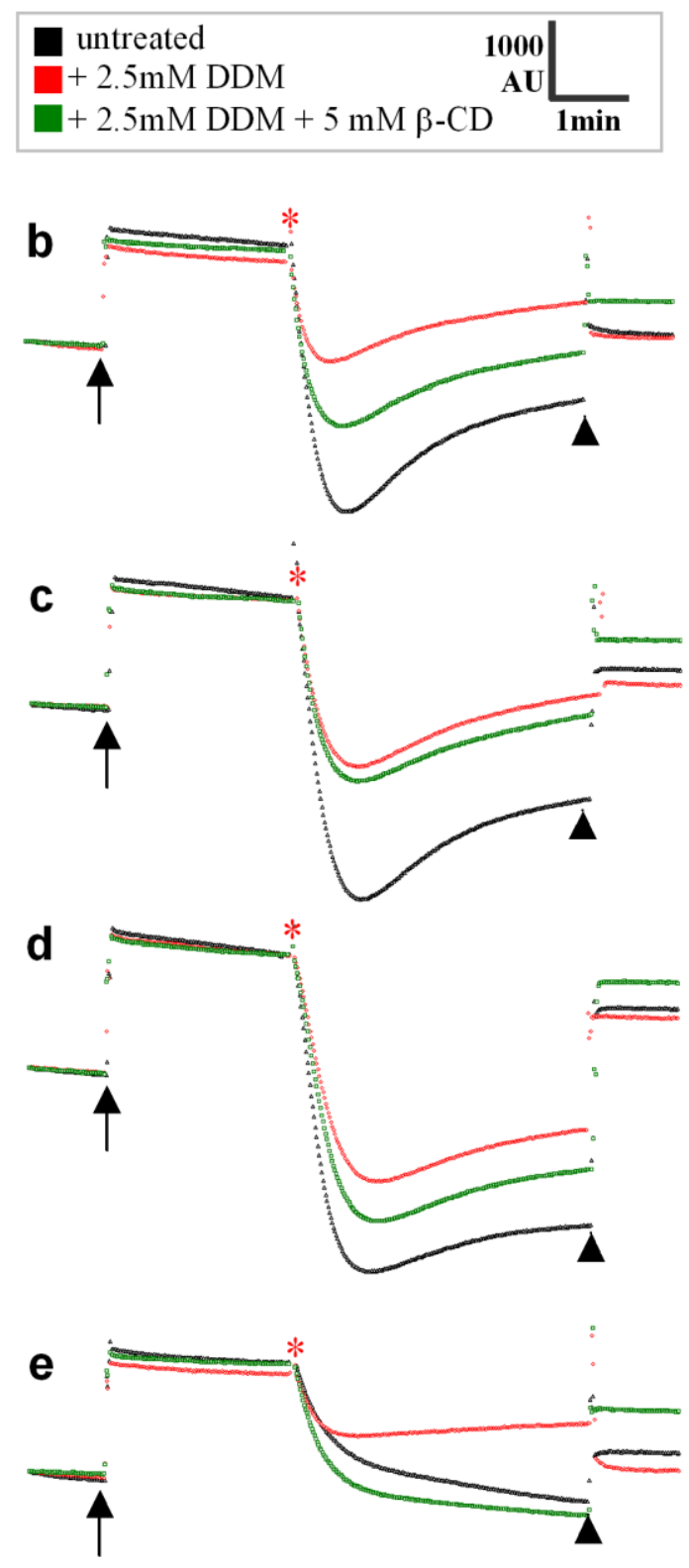

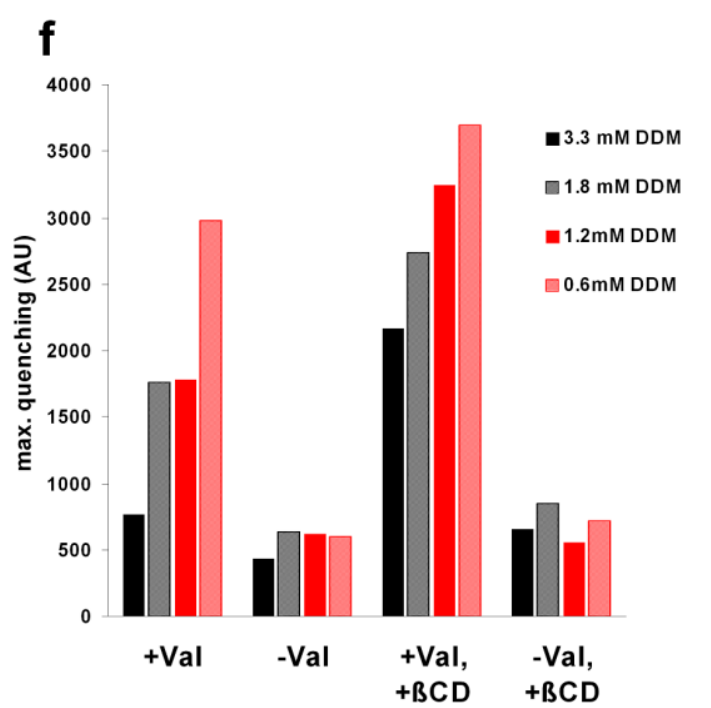

Figure 3.16

Influence of DDM, cholesterol and $\beta-C D$ on proton coupling on reconstituted $T F_{0} F_{1}$ liposomes.

(a) Legend for (b-e) (b-e) AO-quenching traces of $\mathrm{TF}_{0} \mathrm{~F}_{1}$ reconstituted in symmetrical $\mathrm{KCl} 100 \mathrm{mM}$ solution (b-d) or K-Acetate buffer $(100 \mathrm{mM})(\mathrm{e})$. The liposomes contain either no cholesterol (b) , $10 \%$ cholesterol (c) or $30 \%$ cholesterol $(\mathrm{d}, \mathrm{e}, \mathrm{f})$ and are treated with DDM (red), DDM+ $\beta-C D$ (green) or left untreated (black). Addition of ATP, VAL and FCCP are marked with an arrow, red asterisk and an arowhead respectively. The addition of cholesterol improves the sealing of liposomes when $\sim 30 \%$. The acidification stays more stable over time and the liposomes are also less sensitive to the detergent DDM. $\beta-C D$ improves the proton coupling by complexing DDM. Note that the extent of VALdependent acidification strongly depends on the internal anion since acetate results in weaker signals (e). (f) Influence of the DDM concentration on acidification. The VAL-dependent acidification is strongly affected by concentrations $>1.5 \mathrm{mM}$ DDM $(2.5 \mathrm{mg} / \mathrm{ml} \mathrm{lipid})$ and can be recovered well up to 1.5 $\mathrm{mM}$ DDM by $\beta$-CD. Since the VAL-independent signal changes are almost not affected by DDM it is likely that the permeability of the membrane for protons is affected by DDM. 
Because of these ambiguities of the $\mathrm{F}_{0} \mathrm{~F}_{1}$ preparations from E.coli, $\mathrm{TF}_{0} \mathrm{~F}_{1}$, was tested. (Suzuki et al., 2002). It was purified as described in 2.3.2 and reoconstituted in the same way as $\mathrm{F}_{0} \mathrm{~F}_{1}$. The direct comparison in figure 3.15 exemplifies the superiority of this purification over the conventionally purified $\mathrm{F}_{0} \mathrm{~F}_{1}$. Luminal acidification in the absence of valinomycin is almost negligible, while the establishment of a strong $\Delta \mathrm{pH}$ upon addition is still preserved. Therefore this preparation should be well suited to unambigiously detect a newly introduced $\mathrm{Cl}^{-}$conductance from a co-reconstituted protein of interest and thus $\mathrm{TF}_{0} \mathrm{~F}_{1}$ was chosen for all subsequent experiments.

Since DDM will be introduced together with VGLUT1 into the reconstitution mix at concentrations far above the $\mathrm{CMC}$ it was tested up to which concentration this detergent could be tolerated in the liposome suspension without altering the properties for the AOassay (this would somewhat limit the maximal VGLUT1 concentration that can be used in the assay).

Recently, the use of cyclodextrin-derivatives for the formation of liposomes has been reported (Degrip et al., 1998). Cyclodextrins are regularly used for the depletion of cholesterol from cell membranes, which would not be desirable here. However, some cyclodextrins have much higher affinity for long chain amphiphilic molecules such as DDM. Especially 2,6-di-O-methyl- $\beta$-cyclodextrin is suited for removal of DDM from micellar solutions and/or saturated liposomes (Degrip et al., 1998). This approach was tested also for the reconstitution of $\mathrm{TF}_{0} \mathrm{~F}_{1}$ and VGLUT1. A series of experiments was conducted to test how much DDM could be tolerated in the membrane and if cyclodextrins can reverse any negative influence without affecting the assay (Fig. 3.16a-f). The molar ratio of proton-pump to transporter was chosen to be in the range of 1:5 - 1:10 based on the ratio of V-ATPase: VGLUT1 in SVs as guideline (Takamori et al., 2006). The proton pump:lipid ratio should be in the range of 1:50-1:100 (w/w) based on own experimental findings for reasonably strong signal changes in the AO assay. Thus it was roughly known how much detergent will be introduced into the system because the VGLUT1 solution contained approximately $1 \%$ DDM. The parameter that could be altered to stay in an acceptable range of detergent concentration was the total lipid concentration (simply by adjusting the volume of the sample). However a lipid concentration below $1 \mathrm{mg} / \mathrm{ml}$ did not yield very dense liposomes and the sample volume would require concentration of the liposomes after formation (e.g. centrifugation) to be useful in subsequent experiments. 


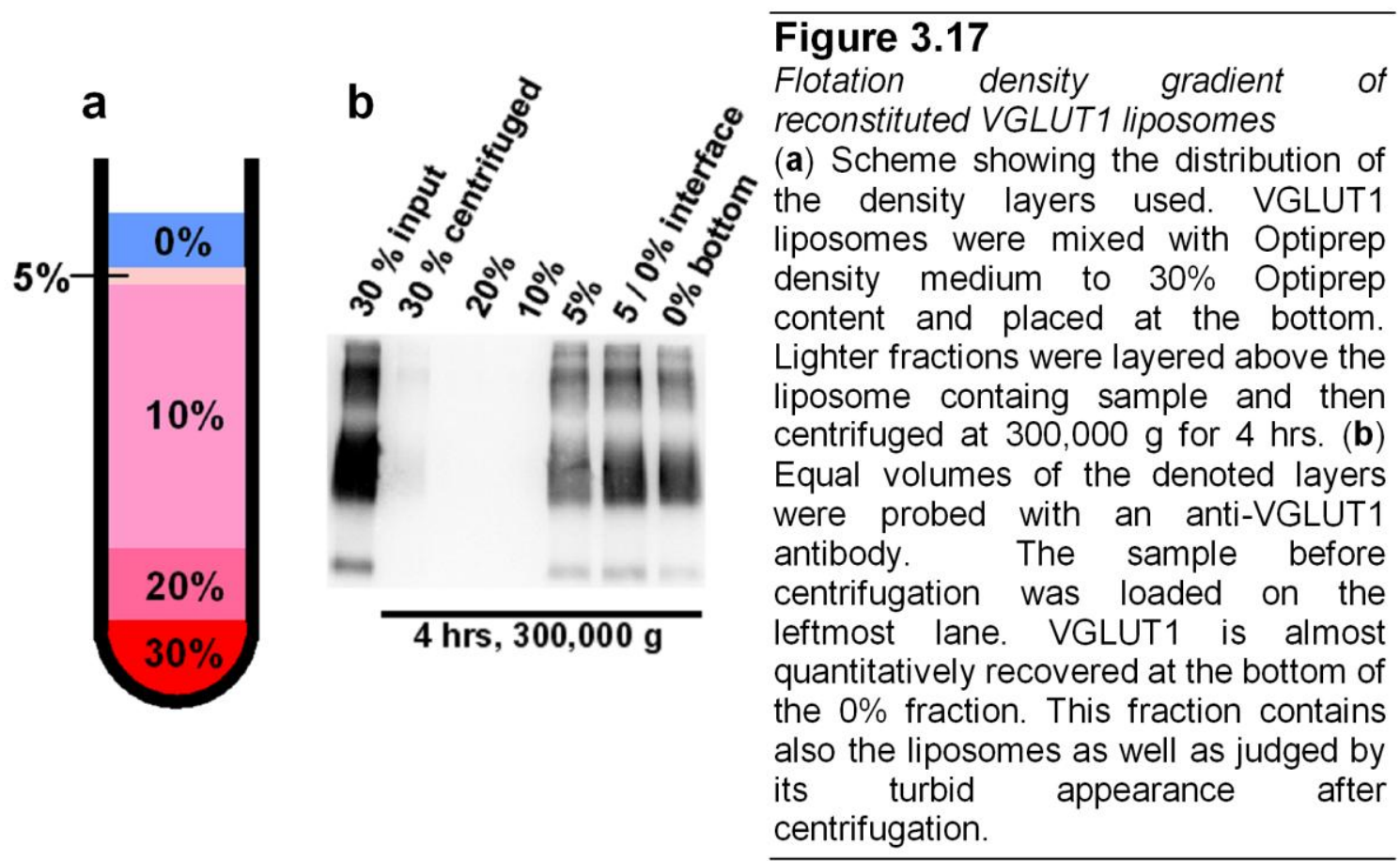

A total lipid concentration of $2.5 \mathrm{mg} / \mathrm{ml}$ was thus a good compromise between the liposome density (sample volume) and detergent concentration.

It has been reported that artficial bilayers with a co-reconstituted V-ATPase require cholesterol to to enable efficient proton coupling (Xie et al., 1986), in similar concentrations as they are reported for SVs (Takamori et al., 2006). It was therefore also tested if this is true for a mixture of phospholipids from soy-beans, $\mathrm{TF}_{0} \mathrm{~F}_{1}$ and different amounts of cholesterol.

The results of these experiments are exemplified in figure 3.16. It can be seen that the use of cholsterol at concentrations of about $30 \mathrm{~mol} \%$ results in good proton coupling and increases the tolerance of the membrane for DDM (compare Fig. 3.16b and 3.16d). Additionally the recovery of the signal after addition of the proton uncoupler FCCP is more pronounced.

\subsection{Co-reconstitution of VGLUT1 and $\mathrm{TF}_{0} \mathrm{~F}_{1}$ and acidification of liposomes}

In order to verify that the $\mathrm{Cl}^{-}$conductance is indeed an intrinsic property of VGLUT1, measurement of $\mathrm{AO}$ quenching in reconstituted liposomes bearing a proton pump and the transporter were necessary. As described above, reasonably pure transporter and ATP- 
a

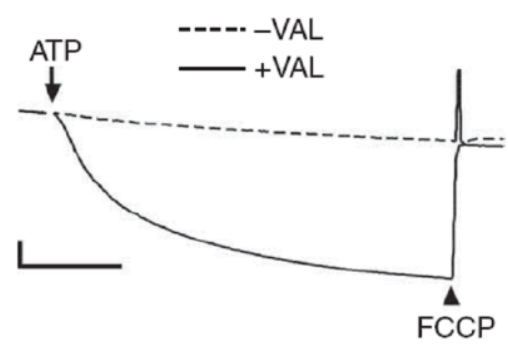

b
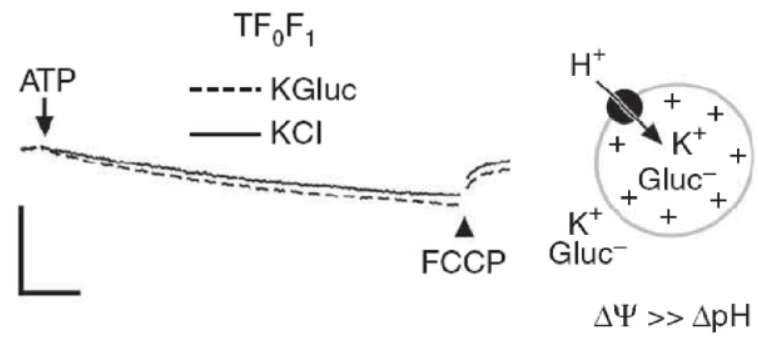

C
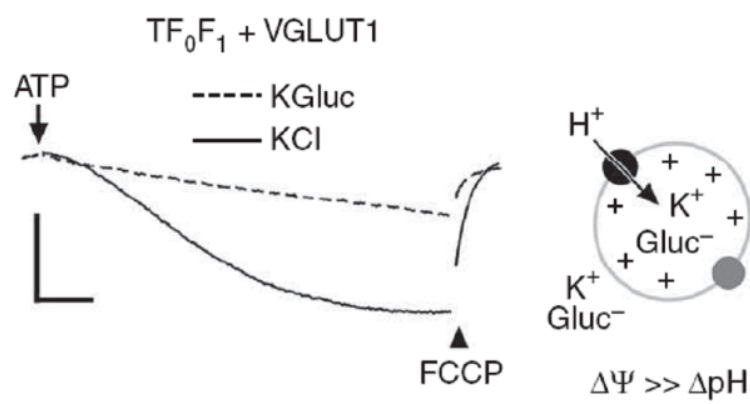

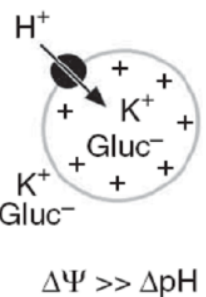

$\mathrm{H}^{+}$

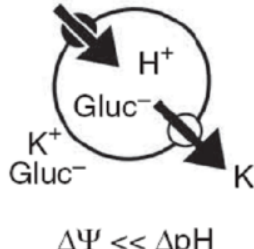

$\Delta \Psi \ll \Delta \mathrm{pH}$

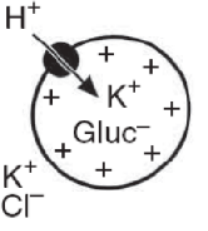

$\Delta \Psi>\Delta \mathrm{pH}$

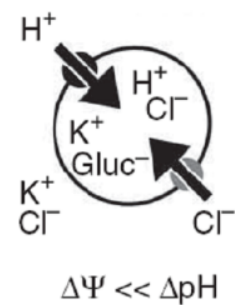

Figure 3.18

Purified VGLUT1 exhibits a chloride conductance

(a) Electrogenic properties of reconstituted $\mathrm{TF}_{0} \mathrm{~F}_{1} \quad\left(\begin{array}{lll}5 & \mu \mathrm{g}\end{array}\right)$. Efficient proton coupling appears only in the presence of the $\mathrm{K}^{+}$-ionophore valinomycin (VAL, solid line).

Re-presentative traces are shown. The sketch on the right illustrates ion movements. $\mathrm{TF}_{0} \mathrm{~F}_{1}$ is represented by black filled circles and VAL by open circles. Strength of $\Delta \Psi$ is symbolized by $(+)$ and low $\mathrm{pH}$ by $\left(\mathrm{H}^{+}\right)$. (b) Acidification in control liposomes containing only $\mathrm{TF}_{0} \mathrm{~F}_{1}$ in the presence of gluconate (dashed) or $\mathrm{Cl}^{-}$ (solid line). (c) Acidification in liposomes coreconstituted with $\mathrm{TF}_{0} \mathrm{~F}_{1}$ and VGLUT1 in gluconate (dashed) or $\mathrm{Cl}^{-}$(solid line). Sketches are labeled as in (a). The gray circle symbolizes VGLUT1.

Scale bars are $500 \mathrm{AU}$ and 120 s respectively.

synthase were obtained in sufficient amounts. Also, a method to prepare well-sealed liposomes was established by evaluation of proton coupling with VAL and the use of the chelating agent $\beta$-CD (Figs. 2.5, 3.4.3). The purified transporter was efficiently reconstituted into liposomes as judged by flotation on Optiprep (Invitrogen) density gradients (Fig. 3.17), eluted as a single peak in semi-analytical gel filtration (Fig. 3.21) without signs of aggregation and exhibited biological activity as described below in detail. The reconstitution of VGLUT1 together with $\mathrm{TF}_{0} \mathrm{~F}_{1}$ has thus proven to be straightforward.

\subsubsection{VGLUT1 exhibits a $\mathrm{Cl}^{-}$conductance in reconstituted liposomes}

$\mathrm{TF}_{0} \mathrm{~F}_{1}$ ATP-synthase acidified lumina of liposomes in symmetric potassium gluconate (KGluc) solution only when free counter ion movement was possible by addition of the $\mathrm{K}^{+}$ 
ionophore (VAL) (Fig. 3.18a) in analogous manner as seen in figures $3.15-3.16$. The electrogenic properties of this $\mathrm{TF}_{0} \mathrm{~F}_{1}$ preparation resemble the V-ATPase (Xie and Stone, 1986) and are therefore suitable to examine the conductance in VGLUT1. Coreconstitution of VGLUT1 and $\mathrm{TF}_{0} \mathrm{~F}_{1}$ into liposomes indeed revealed a conductance for $\mathrm{Cl}^{-}$in VGLUT1 (Fig. 3.18c) as evident from acidification, but not for a bulky anion like gluconate. It is also evident from this experiment that the transporter does not have a conductance for a cation like $\mathrm{K}^{+}$(Fig. 3.18c). Control liposomes bearing only $\mathrm{TF}_{0} \mathrm{~F}_{1}$ did not acidify when external $\mathrm{Cl}^{-}$was present (Fig. 3.18b), clearly showing the impermeability of the vesicle membrane for this anion in the absence of a proteinaceous shunting pathway. A quantitative summary of this experiment is shown in figure 3.19a.

\subsubsection{The $\mathrm{Cl}^{-}$permeabilty of reconstituted liposomes is VGLUT1-dose- dependendent}

A potential pitfall in this approach might be an influence of the reconstituted transporter on the liposomes in way that increases the AO signal independently of a proteinaceous conductance, e.g. due to bias on the liposome volume or by exposing charges to the lumen which in turn effects the trapping or fluorescence of AO. While there is no hint in the scientific literature that would describe such an influence systematically it is nevertheless difficult to fully exclude such a possibility. Unfortunately, there appears to exist no ionophore that equilibrates $\mathrm{Cl}^{-}$ions across lipid bilayers without influence on the $\mathrm{pH}$. Also, no drug is known that would selectively inhibit the $\mathrm{Cl}^{-}$shunt of SVs (DIDS also effects TF0F1 at typical working ranges of 10-50 $\mu \mathrm{M}$, not shown). Therefore, increasing amounts of VGLUT1 were reconstituted and the acidification was measured depending on $\mathrm{Cl}^{-}$and in $\mathrm{Cl}^{-}$-free medium with the addition of VAL (Fig. 3.19b). It was found that reconstitution of VGLUT1 did not affect the properties (AO flurorescence) of the liposomes except for the introduced $\mathrm{Cl}^{-}$conductance since the addition of $\mathrm{VAL}$ in symmetric gluconate solution did not vary upon reconstitution of increasing amounts of VGLUT1. Therefore the potential to quench the fluorescence of $\mathrm{AO}$ in both types of liposomes must be similar. Increasing amounts of VGLUT1 also increased the $\mathrm{Cl}^{-}$conductance but it is not possible to say wether this is due to more $\mathrm{Cl}^{-}$pathways per liposome or if simply more liposomes bear a VGLUT1 molecule, since the number of liposomes is not known. 
a

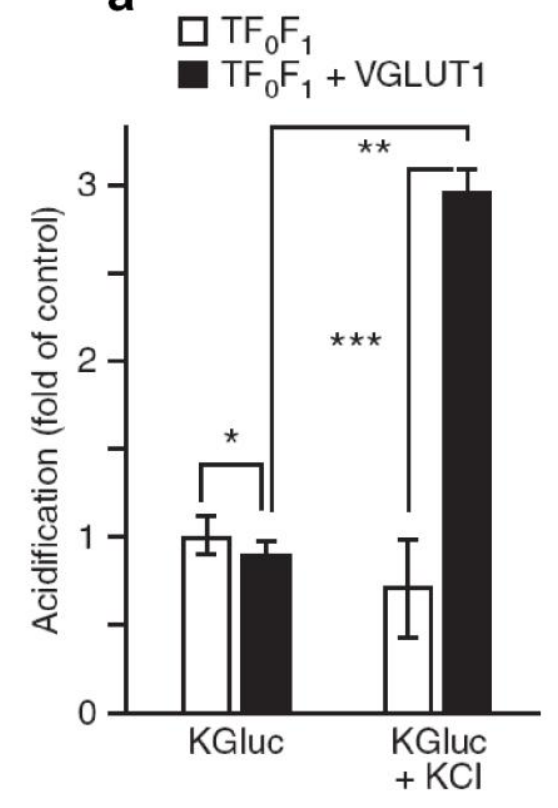

b

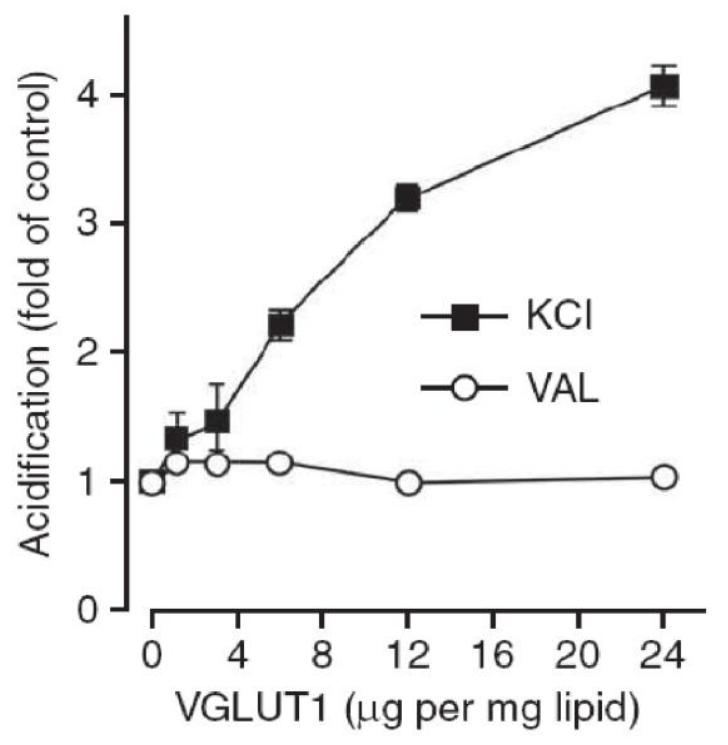

Figure 3.19

Purified VGLUT1 exhibits a chloride conductance (2)

(a) Quantitative summary of the traces in Fig 3.18 (b and c) normalized to the acidification of $\mathrm{TF}_{0} \mathrm{~F}_{1}$ liposomes in symmetric KGluc buffer (ctrl.). Data was analyzed with two-tailed paired $\left({ }^{* *} p<0.001\right)$, and two-tailed unpaired t-test $\left({ }^{*} p>0.3,{ }^{* * *} p<0.005\right)$. (b) $\mathrm{Cl}^{-}$influx is VGLUT1-dose dependent as shown by increasing concentrations of VGLUT1. The VALdependent acidification (single measurements) is not affected. Values are normalized to the mean acidification of $\mathrm{TF}_{0} \mathrm{~F}_{1}$-liposomes without VGLUT1 in the presence of $100 \mathrm{mM}$ external $\mathrm{KCl}$ (filled squares) or $100 \mathrm{mM}$ symmetric KGluc buffer containing VAL (open circles).

\subsubsection{Removal of VGLUT1 of the liposome premix strongly reduces the $\mathrm{Cl}^{-}$ conductance}

While VGLUT1 did not seem to alter the properties of the liposome except for the Clconductivity it appeared still possible that a copurified component from the tsA201 cells could cause the effect. Due to the lack of a specific inhibitor, major contaminations of 
a

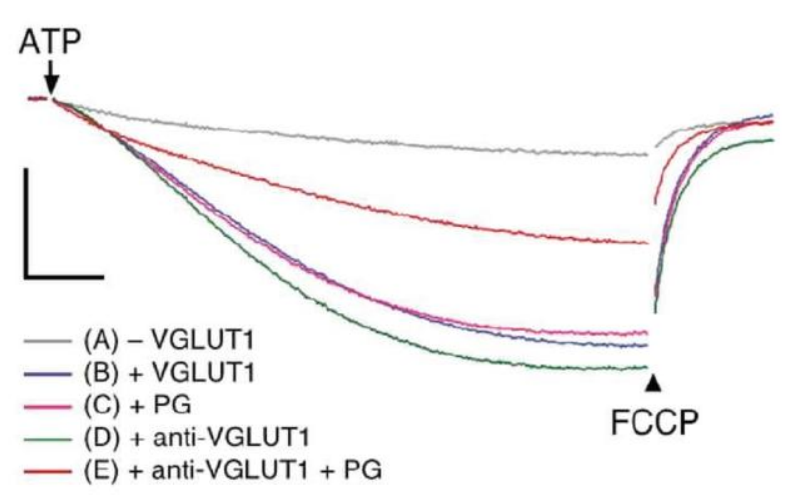

Figure 3.20

Specific removal of VGLUT1 from the liposome premix reduces the $\mathrm{C} \Gamma$ conductance.

(a) VGLUT1 was specifically reduced in the micellar solution prior to liposome formation by an affinity purified polyclonal antibody for VGLUT1 and Protein-G Sepharose beads (E). The resulting acidification in these liposomes was reduced by half compared to untreated sample (B). Treatment with Protein-G Sepharose beads or antibody without removal by Protein- $G$ beads caused no reduction in acidification (C, D). (b) Quantification of the measurements in (a) and analysis of the VGLUT1 content in the different samples by western blotting (guinea pig antiVGLUT1) and silver staining (VGLUT1 and $\alpha / \beta / \gamma$ subunits of $\mathrm{TF}_{0} \mathrm{~F}_{1}$ are indicated). The remaining acidification in sample $(\mathrm{E})$ can be explained by the incomplete removal of VGLUT1 as evident by immunodetection. Independent two-tailed t-test was applied $\left({ }^{*} p<0.01,{ }^{* *} p>0.1\right)$. b

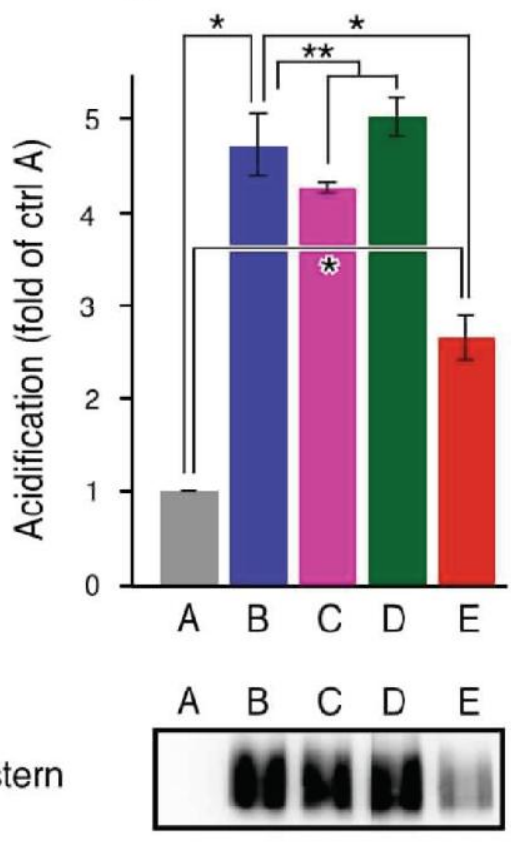

A $\quad$ B $\quad C \quad D \quad E \quad \begin{gathered}\text { M.W. } \\ (\mathrm{kDa})\end{gathered}$

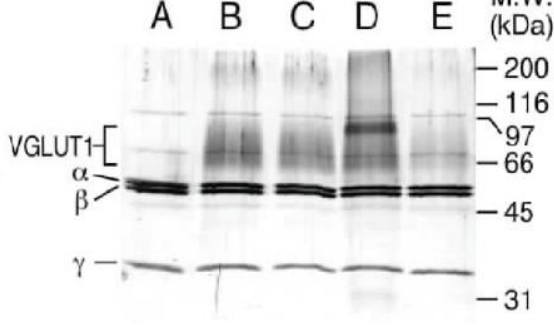

the VGLUT1 preparation were ruled out as a source of the observations, by removal of the transporter from the liposome premix with an affinity purified antibody. For this, excess amount of affinity purified rabbit anti-VGLUT1 antibody was added to a the liposome premix before the liposome-forming step, incubated for 30 minutes and then pulled down with Protein $\mathrm{G}$ beads, after which liposome formation was started by dialysis. Then the acidification was measured of these VGLUT1-depleted liposomes (which could however still contain an unknown contaminant) along with control samples that were prepared in parallel experiments (Fig 3.20). It was revealed that addition of Protein $G$ beads or antibody alone did not alter the acidification of liposomes but only the pulldown of the antibody-VGLUT1 complex on the bead surface reduced the acidification significantly as compared to control liposomes. However, the vesicles still acidified to a greater extent than 
control liposomes which were initially devoid of VGLUT1. Immunodetection with a guinea pig anti-VGLUT1 antibody (Fujiyama et al., 2001) revealed that still a significant amount of VGLUT1 protein was present in the treated liposomes (Fig 3.20b). This was also seen in silver staining (Fig 3.20b, bottom). Although the experiment was repeated, it has proven diffcult to deplete all the VGLUT1 protein in a quantitative manner. The second level of specificity introduced by the procedure described here is nevertheless in support of a $\mathrm{Cl}^{-}$conductance that is caused by VGLUT1 itself instead of arising from accompanying contaminants in the tsA201 isolates.

\subsubsection{The $\mathrm{Cl}^{-}$conductivity co-migrates with VGLUT1 in size exclusion chro- matography}

In a second approach to rule out potential contaminants in the VGLUT1 isolates and also to get an impression of the homogeneity of the concentrated sample (eg. aggregates), approximately $300 \mu \mathrm{g}$ of VGLUT1 at a concentration of $\sim 1 \mathrm{mg} / \mathrm{ml}$ were subjected to size exclusion chromatography (SEC) on a 10/30 Superdex 200 coulmn (GE). The SEC was performed on an ÄKTA system at a flow rate of $0.4 \mathrm{ml} / \mathrm{min}$ in $100 \mathrm{mM} \mathrm{KCl}, 13 \mathrm{mM}$ TrisCl, 0.6 mM EDTA, 1mM DTT and 0.05\% DDM (w/v) at pH 7.4 (Fig. 3.21a). Fractions of $300 \mu \mathrm{l}$ were collected and equal volumes were subjected to western blotting and silver staining. Two fractions that flank the peak, a control sample (running buffer), as well as the peak fraction were reconstituted into liposomes (200 $\mu \mathrm{l}$ each, $600 \mu 1$ liposome mix total), dialyzed overnight ( $\beta$-CD was omitted since the DDM content was already very low) in gluconate buffer and then assayed in the fluorometer for a $\mathrm{Cl}^{-}$conductance (AOquenching) by having either $\mathrm{Cl}^{-}$or gluconate in the assay buffer present as described above (Fig. 3.21b). 
a

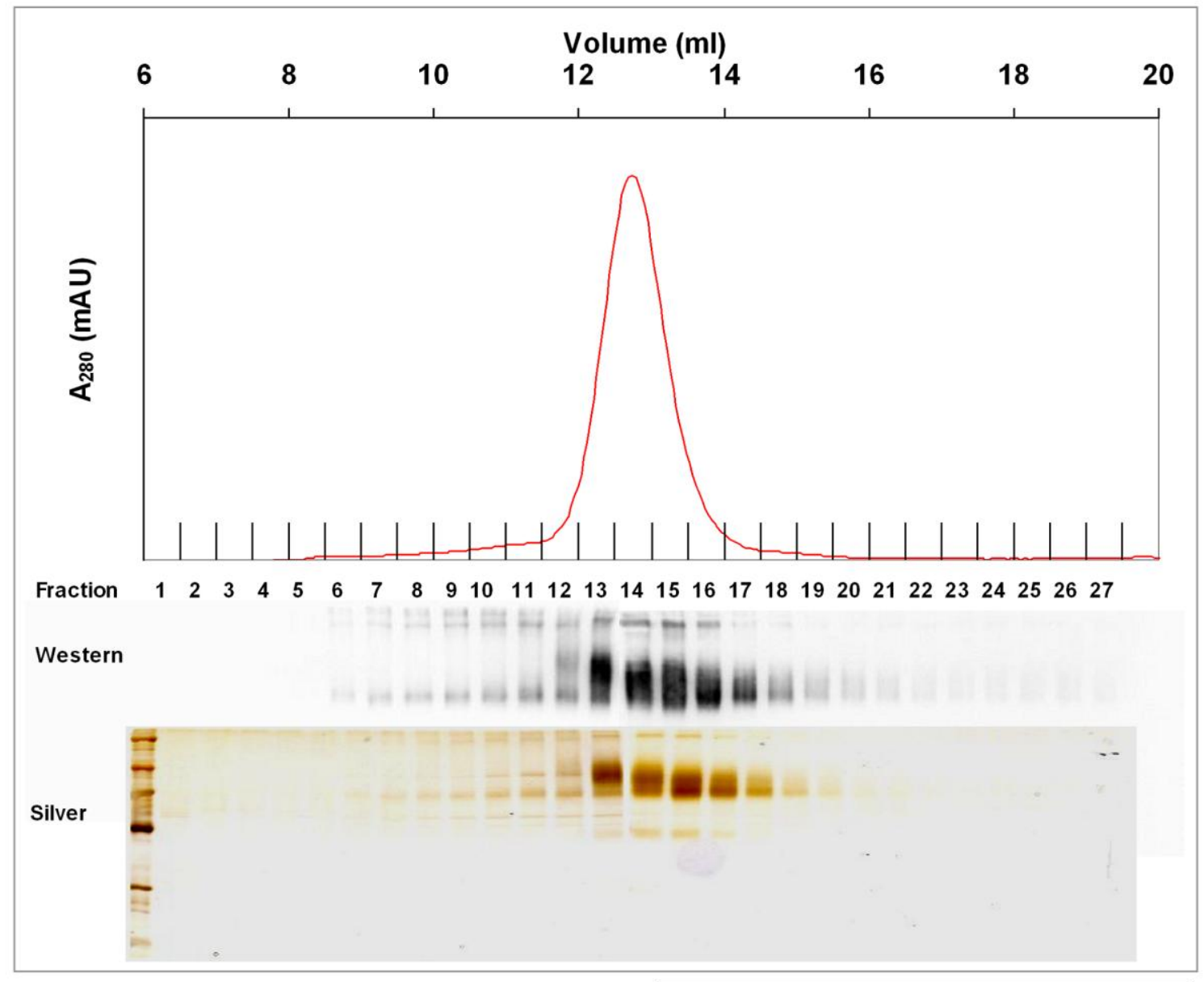

b

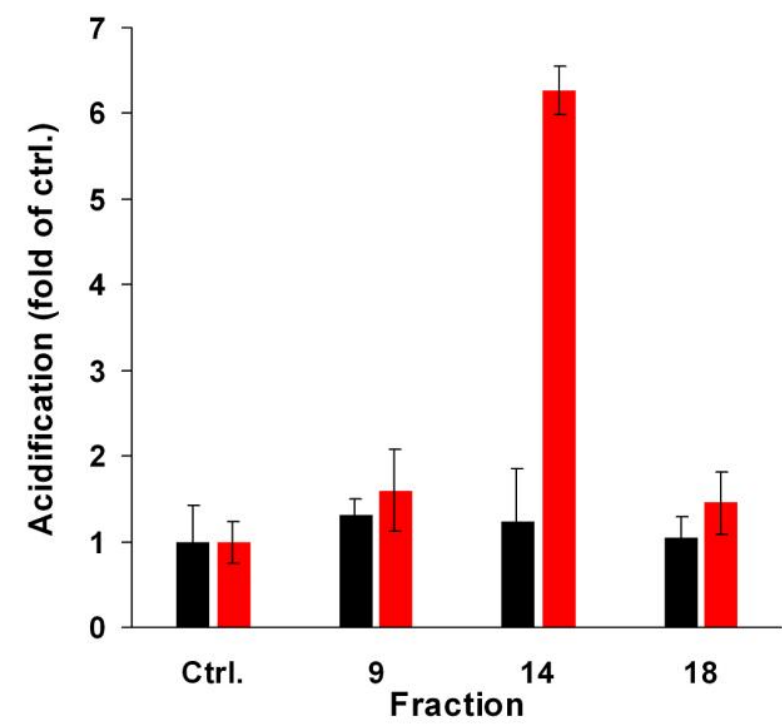

Figure 3.21

Size exclusion chromatography of purified VGLUT1

(a) Purified and concentrated VGLUT1 ( $1 \mathrm{mg} / \mathrm{ml}, \sim 300 \mu \mathrm{g}$ ) was subjected to gel filtration on a Superdex200 10/30 column and eluted with $100 \mathrm{mM} \mathrm{KCl}, 13 \mathrm{mM}$ Tris$\mathrm{Cl}, 0.6 \mathrm{mM}$ EDTA, $1 \mathrm{mM}$ DTT, $0.05 \%$ $\mathrm{DDM}$ (wt/vol), $\mathrm{pH}$ 7.4. Fractions were analyzed by western blotting (top), silver staining (bottom) and reconstitution (b). (b) Fractions 9,14 and 18 from (a) were reconstituted and acidification in the presence of either gluconate (black) or $\mathrm{Cl}^{-}$ (red) measured by $\mathrm{AO}$ quenching (see 2.6.2) was measured. Data are normalized to a control where the running buffer used in (a) was included in the reconstitution mixture instead of eluate. 
It is evident that VGLUT1 elutes as a symmetrical peak with very few large aggregates. The void volume of this column is $\sim 8 \mathrm{ml}$, where large aggregates, not unusual after concentration of proteins, would elute. The western blot and silver stain support the view of a sample that is very homogeneous. It is also apparent from the silver stain that the sample is very pure. The peak would probably be narrower if the glycosylation was more uniform. The western blot and and silver stain show slightly different molecular weights in fractions corresponding to the front of the peak (fractions 12-13, Fig. 3.21a) and its end (fractions 15-17, Fig. 3.21a) which are likely due to different glycosylation patterns. This also supports the idea that VGLUT1 is migrating as a monomer since any oligomeric state would presumably result in a combination of all molecular weights present in the sample and thus not result in a separation with SEC.

The reconstitution of the samples clearly shows that the $\mathrm{Cl}^{-}$conductance is associated with the major peak, thus very likely VGLUT1. A small chance of a contaminant with a similar molecular weight remains but together with the data shown in figure 3.20, this experiment further supports the idea of a $\mathrm{Cl}^{-}$conductance in VGLUT1.

The molecular weight could be estimated in a more precise manner with a molecular weight marker mix but the large micelle of DDM associated with VGLUT1 makes an accurate measurement difficult.

\subsubsection{The $\mathrm{Cl}^{-}$conductance in VGLUT1 is largely independent of the intravesicular anion}

The luminal anion (the major anion in the dialysis buffer during liposome formation) normally used was gluconate. It was thus tested if chloride shunting is still possible with other internal anions, especially $\mathrm{Cl}^{-}$, where a chemical gradient might lower a $\Delta \psi$-driven influx of $\mathrm{Cl}^{-}$. Thus gluconate was replaced with acetate or $\mathrm{Cl}^{-}$and the acidification in the presence of $100 \mathrm{mM}$ external $\mathrm{Cl}^{-}$was tested (Fig 3.22). It is difficult to quantify the differences in this assay since the results can not be normalized with VAL, as this would result in dramatically different signals (see Fig. 3.16), depending on the internal anion. However, from a qualitative point of view it appears that $\mathrm{Cl}^{-}$-loaded liposomes still acidify in the presence of external $\mathrm{Cl}^{-}$with the same concentration $(100 \mathrm{mM})$ and therefore build up a chemical gradient. A similar finding has been made with a reconstituted putative 
chloride channel from bovine clathrin coated vesicles which was also tested in symmetric $\mathrm{KCl}$ solution (Xie et al., 1989). The vectoriality for the $\mathrm{Cl}^{-}$flux must therefore be determined by the membrane potential, and is probably only to some extent influenced by the chemical gradient since only very few $\mathrm{Cl}^{-}$ions enter the lumen. Potentially the influx into $\mathrm{Cl}^{-}$-loaded liposomes is lowered if the external $\mathrm{Cl}^{-}$concentration is in the low millimolar range, but later experiments, using Nigericin, speak against it (see 3.65).

a

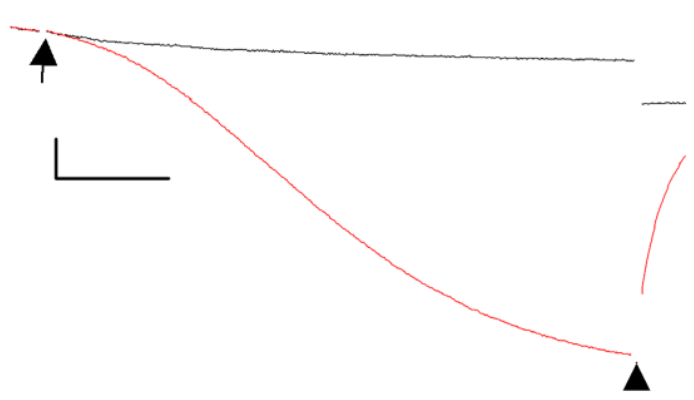

b

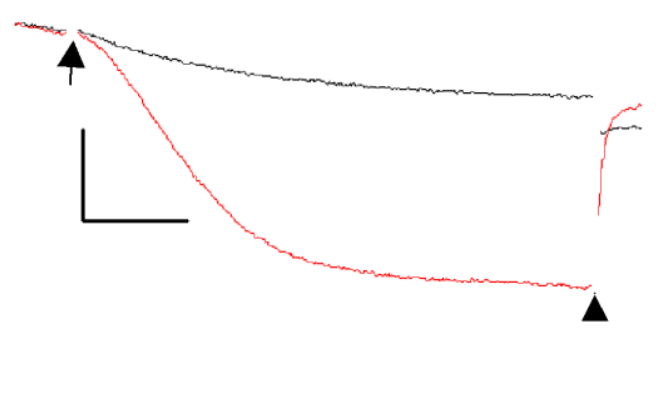

\section{Figure 3.22}

$\mathrm{Cl}$ shunting occurs independently of the internal anion

$(\mathbf{a}, \mathbf{b})$ VGLUT1 containing vesicles were reconstituted in the presence of acetate (a) or $\mathrm{Cl}^{-}$ (b) $(100 \mathrm{mM})$ and acidification was subsequently tested in $100 \mathrm{mM}$ external gluconate (black) or $100 \mathrm{mM}$ external $\mathrm{Cl}^{-}$(red). Please note that the gluconate measurement in (b) contains also $8 \mathrm{mM} \mathrm{Cl}^{-}$since the external salt of the liposomes was not exchanged by gelfiltration after dialysis and is thus introduced by the application of the sample into the cuvette. The scale bars represent $500 \mathrm{AU}$ and 120 s respectively. Single traces are shown. Arrows indicate addition of ATP and arrowheads application of FCCP. 


\subsection{Glutamate uptake of VGLUT1 - $\mathrm{TF}_{0} \mathrm{~F}_{1}$ liposomes}

\subsubsection{The intravesicular ion composition is an unknown factor for glutamate transport}

Having verified that VGLUT1 is responsible both for glutamate transport and for $\mathrm{Cl}^{-}$ permeability in liposomes and SVs, it was a logical consequence to test in detail how the $\mathrm{Cl}^{-}$conductance in VGLUT1 influences glutamate transport. To date, only effects of extravesicular $\mathrm{Cl}^{-}$could be investigated using native vesicles but those of intravesicular $\mathrm{Cl}^{-}$ have never been examined experimentally. However, an intuitive assumption is that SVs engulf extracellular fluid during endocytosis, and therefore should be filled with $\sim 130 \mathrm{mM}$ $\mathrm{NaCl}$. The passive capture of molecules/ions has indeed been demonstrated at the presynaptic terminal due to recycling of SVs by exo-/endocytosis, making it very hard to imagine that a small anion like $\mathrm{Cl}^{-}$could be efficiently excluded (although some modification of the fluid is imaginable from fixed Donnan charges on the luminal surface of the invaginating SV).

\subsubsection{Glutamate uptake into liposomes depends on ATP and is enhanced by intravesicular $\mathrm{Cl}^{-}$}

To test the scenario described above, the liposomes were preloaded with $100 \mathrm{mM} \mathrm{KCl}$ or $100 \mathrm{mM}$ Kgluc as the major salts, and glutamate uptake was measured in the presence of 4 $\mathrm{mM}$ external $\mathrm{Cl}^{-}$which is known to be optimal for the uptake (Naito and Ueda, 1985) (see Fig. 3.23a for an illustration of $\mathrm{Cl}^{-}$and gluconate distributions). Intriguingly, the ATPdependent uptake of glutamate was strongly enhanced in the presence of luminal $\mathrm{Cl}^{-}$ compared to the impermeable gluconate by a factor of approximately 3 (Fig. 3.23a). Uptake of glutamate was clearly depending on $\Delta \mu \mathrm{H}^{+}$since addition of ATP was essential. The system also revealed a very low background because the signal from liposomes without VGLUT1 was very low, no matter whether ATP was added or not and highlighted that the increase in glutamate uptake in the presence of intravesicular $\mathrm{Cl}^{-}$must be linked to VGLUT1. It should be noted that liposomes containing $\mathrm{Cl}^{-}$led to higher uptake in the absence of ATP than liposomes that where filled with gluconate. This appeared to be 
a

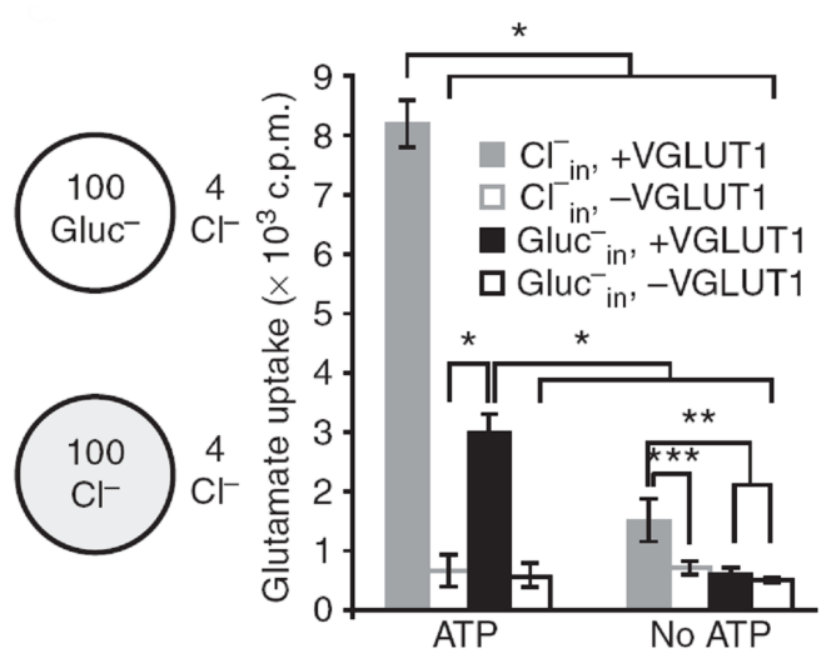

b

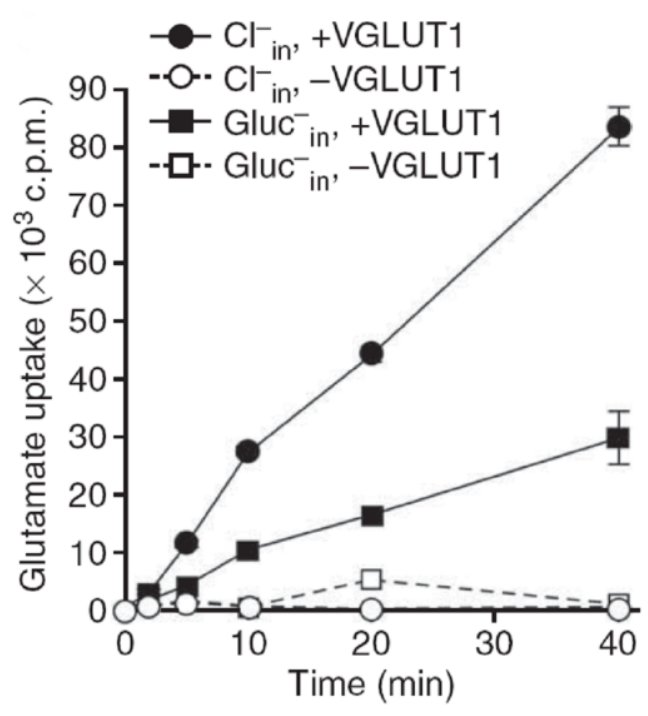

Figure 3.23

Enhancement of glutamate transport into liposomes by high luminal $\mathrm{Cr}$ concentrations (a) Glutamate uptake (12 min) into liposomes preloaded with KGluc (black) and $\mathrm{KCl}$ (gray) under standard conditions (see Methods) with or without addition of ATP. Uptake into liposomes lacking VGLUT1 is shown in open bars. ${ }^{*} p<0.01,{ }^{* *} p<0.05$ and ${ }^{* * *} p=0.053$ (twotailed unpaired t-test). (b) Time course of glutamate uptake into liposomes preloaded with KGluc (squares) and $\mathrm{KCl}$ (circles) under standard conditions. Uptake into liposomes lacking VGLUT1 is shown in the traces with dashed lines (single measurements). A sketch showing the internal and external concentrations in $(\mathrm{mM})$ of $\mathrm{Cl}^{-}$and gluconate used for experiments in this figure can be seen on the left.

the case also when $\mathrm{TF}_{0} \mathrm{~F}_{1}$ was omitted (not shown). However, the uptake was small compared to uptake driven by $\Delta \mu \mathrm{H}^{+}$.

A time course of glutamate uptake indicated an increased velocity as well as a higher loading capacity for $\mathrm{Cl}^{-}$-loaded liposomes (Fig. 3.23b).

\subsubsection{The uptake kinetics show an increase of $v_{\max }$ for $\mathrm{Cl}^{-}$loaded liposomes compared to gluconate loaded liposomes}

The uptake kinetics revealed a substantial increase in $v_{\max }$ (3.3-fold), while the affinities measured for both gluconate- and $\mathrm{Cl}^{-}$-loaded liposomes differ only slightly and approximate reported values $\left(K_{\mathrm{M}} \sim 1-2 \mathrm{mM}\right)$ (Naito and Ueda, 1985; Wolosker et al., 1996) (Fig. 3.24). 
a

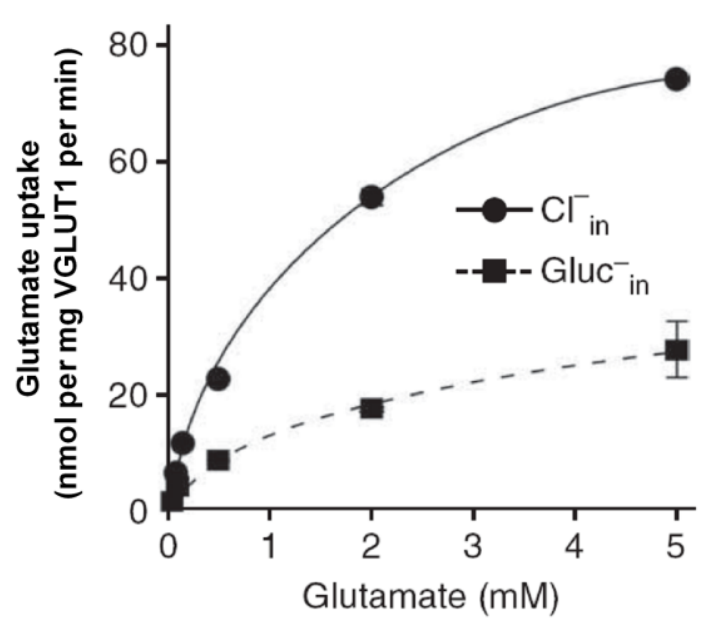

b

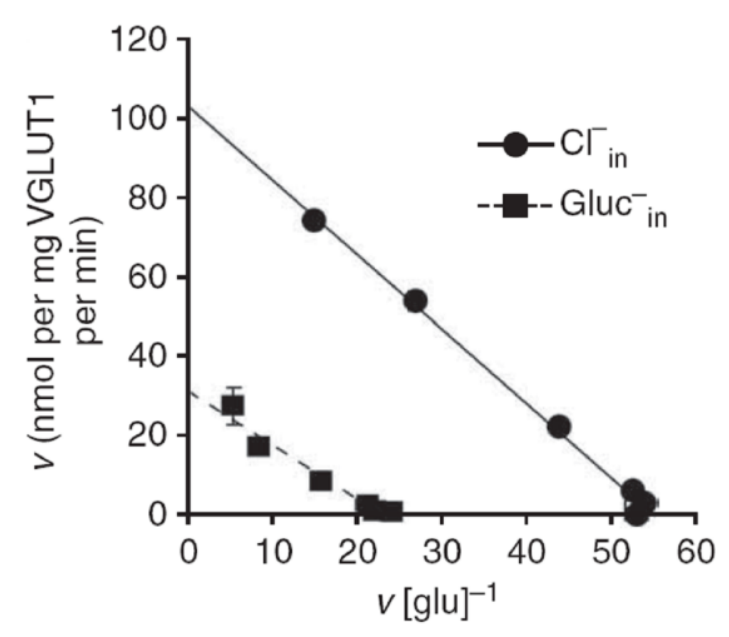

\section{Figure 3.24}

Kinetics of glutamate uptake in the presence of luminal gluconate or $\mathrm{Cr}$

(a) Kinetics of glutamate uptake under standard conditions with liposomes preloaded with $\mathrm{KCl}$ (solid line) and KGluc (dashed line), respectively. Data was taken after 15 min uptake with varying concentrations of non-labeled glutamate. (b) Eadie-Hofstee-Plot of the data in (a). $K_{M}(\mathrm{mM})$ and $V_{\max }(\mathrm{nmol} \mathrm{Glu} / \mathrm{mg}$ VGLUT1/min) are 1.36 \pm 0.19 and 31,21 \pm 4.4 for gluconate-loaded liposomes, and $1.86 \pm 0.03(p<0.05)$ and $102.52 \pm 2.27(p<0.0005)$ for $\mathrm{Cl}^{-}$ -loaded liposomes, respectively (independent two-tailed t-test).

\subsubsection{Influence of extravesicular $\mathrm{Cl}^{-}$on the glutamate uptake in liposomes}

\subsubsection{The biphasic dependence on extravesicular $\mathrm{Cl}^{-}$is preserved in the reconstituted system}

To investigate if the biphasic dependence of glutamate transport on extravesicular $\mathrm{Cl}^{-}$is preserved in this minimal system and can be explained by the conductance in VGLUT1, glutamate uptake was measured in the presence of different external $\mathrm{Cl}^{-}$concentrations for both of the tested internal anions (Fig. 3.25). Interestingly, the dependence on external $\mathrm{Cl}^{-}$ for gluconate-loaded liposomes resembles the pattern observed for isolated SVs (Maycox et al., 1988; Naito and Ueda, 1985) where the uptake is very low in the absence of external $\mathrm{Cl}^{-}$, enhanced by low millimolar $\mathrm{Cl}^{-}$( 6-fold), and attenuated by rising external $\mathrm{Cl}^{-}$ concentrations (Fig. 3.25, black filled diamonds). $\mathrm{Cl}^{-}$-loaded liposomes show also enhanced uptake by low millimolar concentrations of $\mathrm{Cl}^{-}$, albeit weaker ( 1.2-fold) and 
uptake is attenuated by rising external $\mathrm{Cl}^{-}$concentrations in a similar manner (Fig. 3.25, red filled triangles). Most strikingly, however, in the absence of external $\mathrm{Cl}^{-}$the uptake by $\mathrm{Cl}^{-}$-loaded liposomes is about 10-fold higher than for gluconate-loaded liposomes (Fig. $\left.3.25,\left[\mathrm{Cl}^{-}\right]_{\text {out }}=0\right)$.

\subsubsection{2 $\mathrm{Cl}^{-}$loaded liposomes show higher uptake at all extravesicular $\mathrm{Cl}^{-}$ concentrations}

Essentially for all the conditions tested, a higher uptake for the $\mathrm{Cl}^{-}$-loaded liposomes than for the gluconate-loaded liposomes was observed. But this is most obvious under conditions with high $\Delta \Psi$. For uptake with close to physiologically relevant

a

\section{$40 \mu \mathrm{M}$ glutamate}

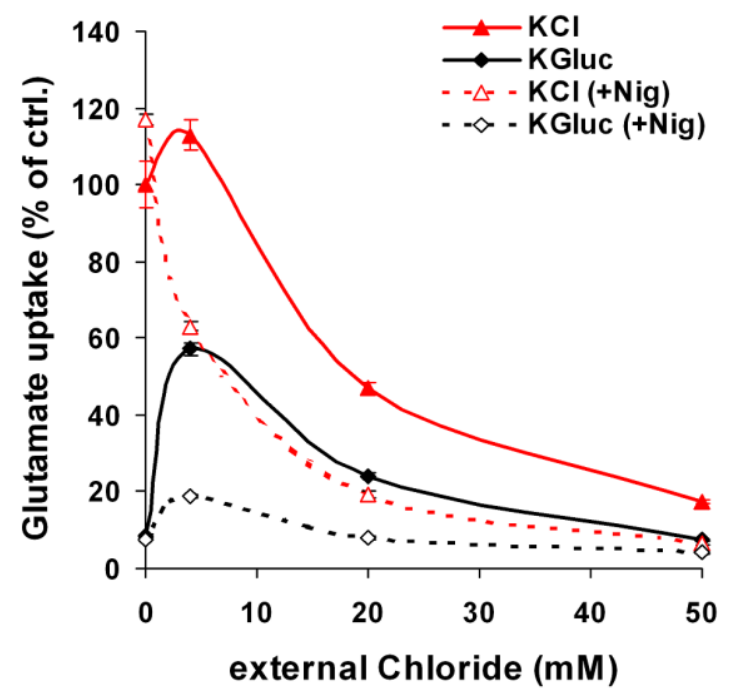

b

\section{$5 \mathrm{mM}$ glutamate}

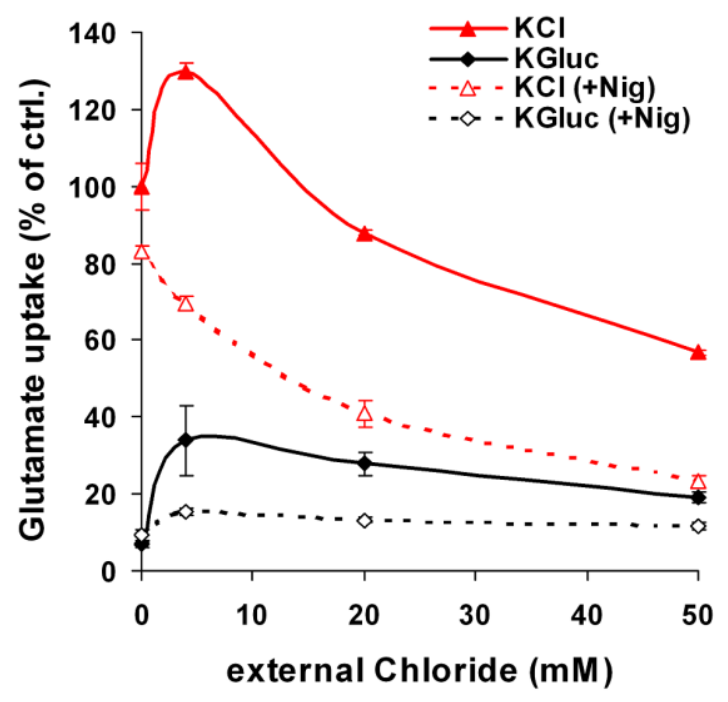

\section{Figure 3.25}

Dependence of glutamate transport on extravesicular $\mathrm{C \Gamma}$ and contribution of $\Delta \mathrm{pH}$ and $\Delta \Psi$ on the total uptake

(a and b) Glutamate uptake (30 min) into liposomes containing KGluc (black diamonds) and $\mathrm{KCl}$ (red triangles) under varying external $\mathrm{Cl}^{-}$concentrations in the absence (solid lines) and presence of NIG (dashed lines). Uptake with either $40 \mu \mathrm{M}$ glutamate (a) or $5 \mathrm{mM}$ glutamate (b) was assayed. The data was normalized to a control (100 $\mathrm{mM} \mathrm{Cl}_{\text {in }}^{-}, 0 \mathrm{mM} \mathrm{Cl}_{\text {out }}^{-}$no NIG).

concentrations of glutamate $(5 \mathrm{mM})$, the difference in uptake between the two tested anions is even bigger (Fig. 3.25, red symbols). 


\subsection{5 $\Delta \psi$-driven uptake is strongly facilitated by intravesicular $\mathrm{Cl}^{-}$}

To evaluate the role of protons in the transport process, the $\mathrm{K}^{+} / \mathrm{H}^{+}$exchanger nigericin (NIG) was used, which selectively dissipates $\Delta \mathrm{pH}$. This made it possible to estimate the relative contributions of either $\Delta \Psi$ or $\Delta \mathrm{pH}$ on the net uptake of glutamate into liposomes (Fig. 3.25, dashed lines). The NIG-resistant uptake, hence driven by $\Delta \Psi$, is much larger if the liposomes are loaded with $\mathrm{Cl}^{-}$, maximal in the absence of external $\mathrm{Cl}^{-}$, and gradually decreasing with raising external $\mathrm{Cl}^{-}$concentrations. In contrast, the transport driven by $\Delta \Psi$ into gluconate-loaded liposomes is very low, albeit minimal uptake at low millimolar concentrations of external $\mathrm{Cl}^{-}$remains. Clearly, $\Delta \Psi$-driven transport of glutamate is only possible when $\mathrm{Cl}^{-}$is present in the lumen. Uptake for gluconate-loaded liposomes appears to be entirely fueled by $\Delta \mathrm{pH}$, indicating an $\mathrm{H}^{+} /$glutamate antiport. It should be noted that this component is also present in $\mathrm{Cl}^{-}$-loaded liposomes and contributes to the total uptake. An additional important conclusion that can be

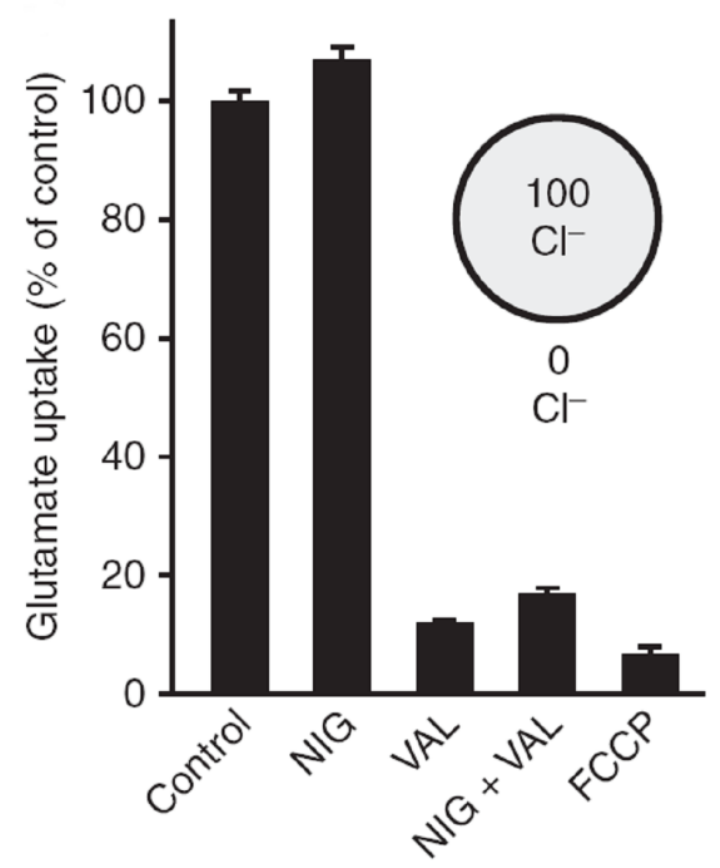

Figure 3.26

$\Delta \Psi$ is indispensable for glutamate uptake in the absence of external $\mathrm{Cr}$

Glutamate uptake in the presence of different ionophores for $\mathrm{Cl}^{-}$-loaded liposomes in the absence of external $\mathrm{Cl}^{-}$was measured for 30 min. NIG, VAL and FCCP were used used at 200nM, $100 \mathrm{nM}$ and $20 \mu \mathrm{M}$ final concentrations. The data are normalized to a control (transport without any additives).

drawn from the effect of NIG is that high external $\mathrm{Cl}^{-}$concentrations do not necessarily reduce uptake by imposing a larger $\Delta \mathrm{pH}$ since this component must be negligible in the presence of the ionophore. This can be seen well for $\mathrm{Cl}^{-}$-loaded liposomes (Fig. 3.25, open red triangles) and indicates competition of $\mathrm{Cl}^{-}$with glutamate for entering the lumen. 


\subsubsection{Glutamate uptake depends on the actual internal $\mathrm{Cl}^{-}$concentration:}

\section{Indications for a glutamate/Cl${ }^{-}$antiport activity in VGLUT1}

The uptake of $\mathrm{Cl}^{-}$-loaded liposomes in the absence of external $\mathrm{Cl}^{-}$was further characterized which demonstrated the striking dependence on the membrane potential. Dissipation of $\Delta \Psi$ by application of VAL abolished the transport (Fig. 3.26). The

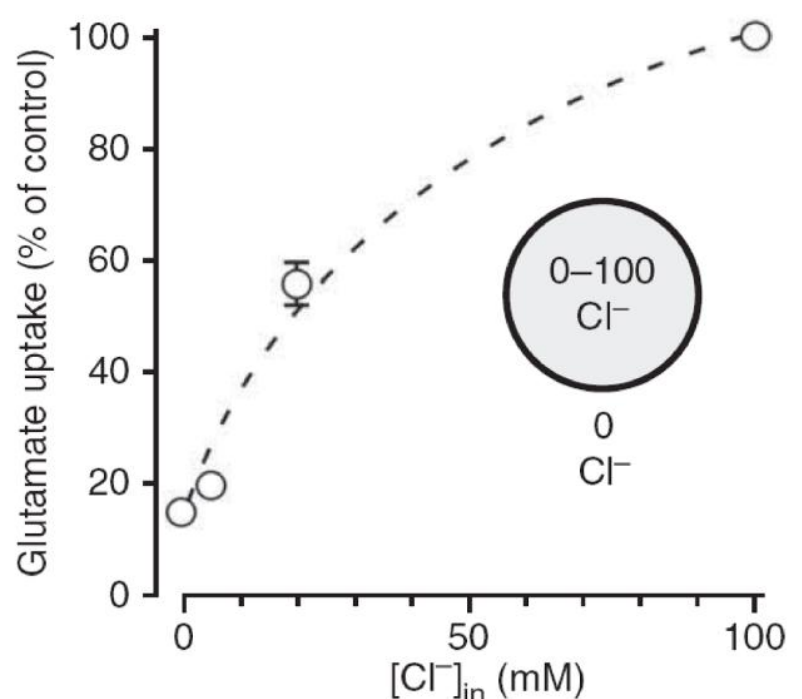

Figure 3.27

Intravesicular $\mathrm{Cl}$ - could be a substrate in an antiport mode of VGLUT1

Glutamate uptake under varying internal $\mathrm{Cl}^{-}$concentrations without external $\mathrm{Cl}^{-}$. Data in the presence of $100 \mathrm{mM}$ internal $\mathrm{Cl}^{-}$was taken as $100 \%$. Note that more than $50 \%$ of uptake remains with only 20 $\mathrm{mM}$ internal $\mathrm{Cl}^{-}$.

transport is consequently strongly reduced by dissipation of $\Delta \mu \mathrm{H}^{+}$(NIG+VAL and FCCP). A strict dependence on the actual internal $\mathrm{Cl}^{-}$concentration (Fig. 3.27) was observed, which indicates that $\mathrm{Cl}^{-}$is a substrate during the transport. Alternatively, the inside positive membrane potential generated by leaking $\mathrm{Cl}^{-}$through

a

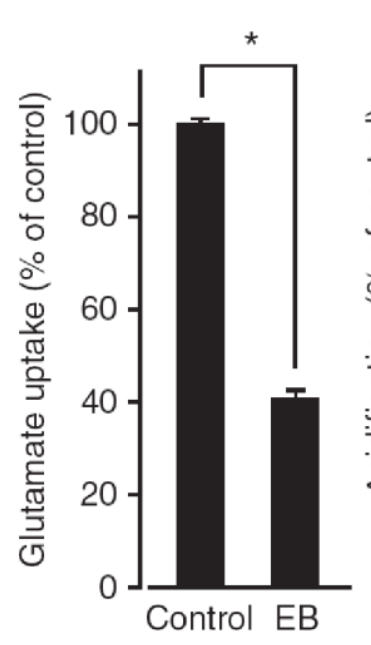

b

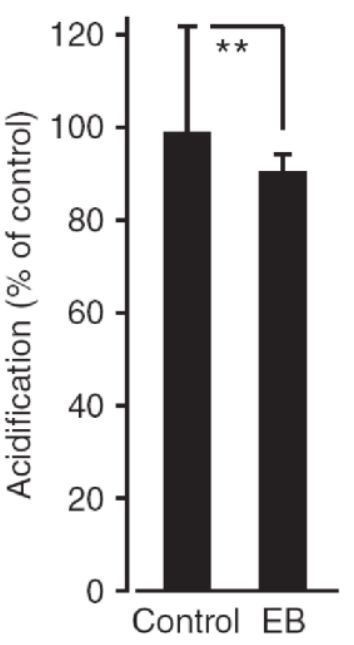

Figure 3.28

Binding of glutamate and $\mathrm{Cl}$ to VGLUT1 is differently affected by Evan's Blue

(a) Effect of $1 \mu \mathrm{M}$ Evan's Blue (EB) on glutamate transport $\left(100 \mathrm{mM} \mathrm{Cl}^{-}\right.$in, $0 \mathrm{mM}$ $\mathrm{Cl}^{-}$out), $30 \mathrm{~min}$ uptake was assayed. (b) Effect of $1 \mu \mathrm{M}$ EB on the acidification of liposomes. Note that the substrate concentrations in the assays differ by the factor $10^{3}$ for glutamate and $\mathrm{Cl}^{-}$ respectively, while EB was used at the same concentration in (a) and (b). Data was analyzed with two-tailed paired t-tests $\left({ }^{*} p<0.001,{ }^{* *} p>0.5\right)$. 
VGLUT1 could also contribute to the driving force. It might explain the slightly elevated uptake in the absence of ATP when compared to gluconate-loaded liposomes (Fig. 3.25), but generation of $\Delta \Psi$ by $\mathrm{TF}_{0} \mathrm{~F}_{1}$ is essential, probably by inducing conformational changes in the transporter that are necessary for substrate translocation (Wolosker et al., 1996).

Uptake was inhibited by $1 \mu \mathrm{M}$ Evan's Blue, a known inhibitor of vesicular glutamate transport (Roseth et al., 1995), by approximately $60 \%$ (Fig. 3.28a), but $\mathrm{Cl}^{-}$-dependent acidification was not affected at the same concentration (Fig. 3.28b). This supports the view of two independent binding sites for glutamate and $\mathrm{Cl}^{-}$on the transporter (Hartinger and Jahn, 1993), but it does not exclude a possible overlap.

\subsection{Comparison of the internal volumes of $\mathrm{Cl}^{-}$- and gluconate loaded liposomes}

The results shown in 3.6 clearly show distinct biophysical characteristics in the transport of glutamate dependening on the internal anion, such as the dependence on $\Delta \psi$ or $\Delta \mathrm{pH}$ or the sensitivity to NIG. Intuitively, however, differences in the volume of the liposomes could come to mind that might explain some of the observations. For example the total capacity for glutamate loading (note that the equilibrium has not been reached in experiments done here) could be a secondary effect of differently sized liposomes. To see if the size varies substantially between gluconate- or $\mathrm{Cl}^{-}$-loaded liposomes the volume of the two populations was estimated by two techniques.

\subsubsection{Electron micrographs reveal only minor differences in the volume of $\mathrm{Cl}$ - and gluconate loaded liposomes}

In a first approach, average liposome diameters were measured in electron micrographs. For this the liposomes were diluted to an appropriate density, spotted on collodion coated nickel grids, fixed and negatively stained with uranyl acetate (see 2.8). Then images were taken in a Hitachi electron microscope at $75 \mathrm{kV}$ and the micrographs were analyzed by measuring the average diameter (mean of shortest and longest axis) of the liposomes with the ImageJ software $(\mathrm{NIH})$. From the mean diameter the corresponding volumes were calculated for $\sim 600$ liposomes. Since the sizes vary substantially the results were displayed 
in a cloud plot. Mean and median values are shown and the difference in size is very small albeit significant (Fig. 3.29)

a

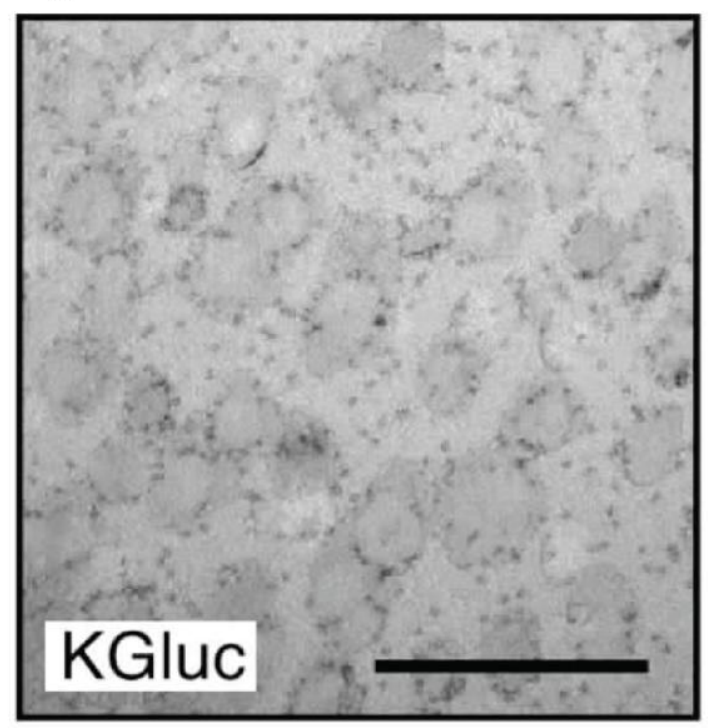

C

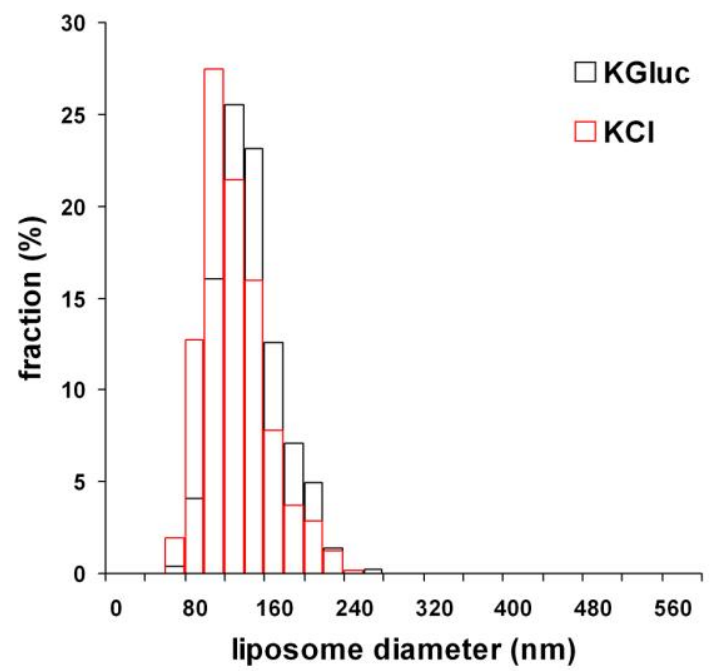

b

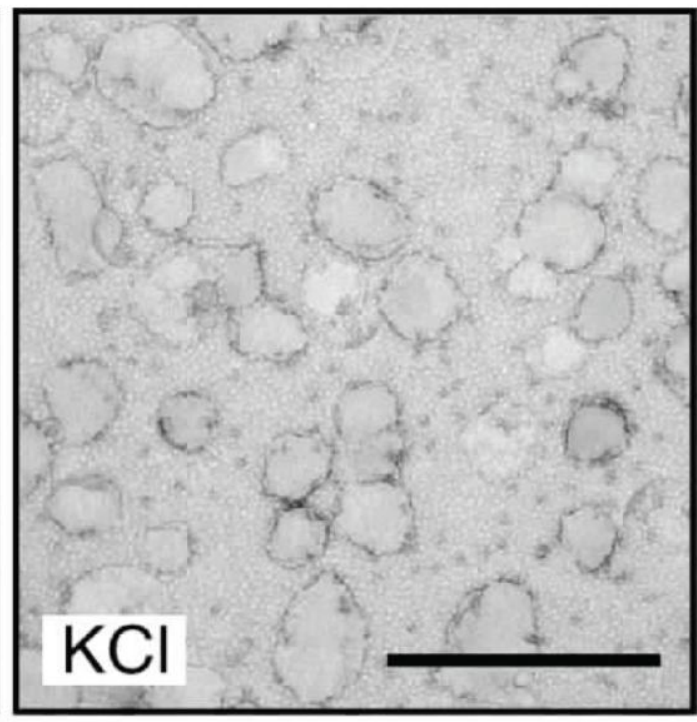

d

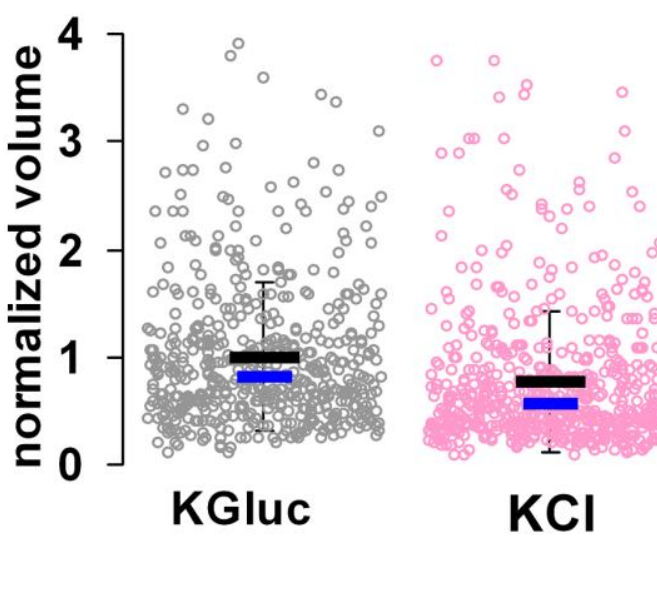

\section{Figure 3.29}

Relative volumes of liposomes formed in $\mathrm{KGluc}$ or $\mathrm{KCl}$ containing buffers

( $\mathbf{a}$ and $\mathbf{b}$ ) Electron micrographs of negatively stained proteoliposomes prepared in KGluc- (a) and $\mathrm{KCl}$-based buffer (b), respectively. The scale bar represents $500 \mathrm{~nm}$. (c) Average diameters of proteoliposomes prepared in $\mathrm{KCl}$ (red) or KGluc (black) measured in electron micrographs. The data is from $\sim 600$ liposomes (10 micrographs) for each sample. (d) Calculated volumes from mean diameters of liposomes in both preparations. Black bars represent the mean with standard deviation and blue bars the median. Individual volumes are plotted as a cloud. Data are normalized to the mean of KGluc liposomes and the $y$-axis represents the Volume (fold of KGluc mean). Data was analyzed with two-tailed unpaired t-test $(p<0.001)$. 


\subsubsection{Direct measurement of the total liposome volume with a radiotracer}

Since the volume determination might suffer from artefacts like different shrinking or uneven adsorption on the grid for small and big liposomes, a second way to estimate the volume was chosen. If an inert detectable substance is included in the dialysis buffer and then encapsulated during the liposome formation, it can be used to determine the relative volume of the two populations after separation of the liposomes from the surrounding buffer, e.g. by gel filtration. The signal that is associated with the liposome fraction after such a separation is then directly proportional to the internal volume. Radiolabelled glucose $\left(\left[{ }^{14} \mathrm{C}\right]\right.$-glucose $)$ is an ideal substance for this approach, since it is uncharged, small, membrane impermeant and the isotope is easily measurable. Due to its uncharged but polar nature it should distribute neutrally between the inside and outside of liposomes during the formation and not interact physically with membrane lipids or proteins.

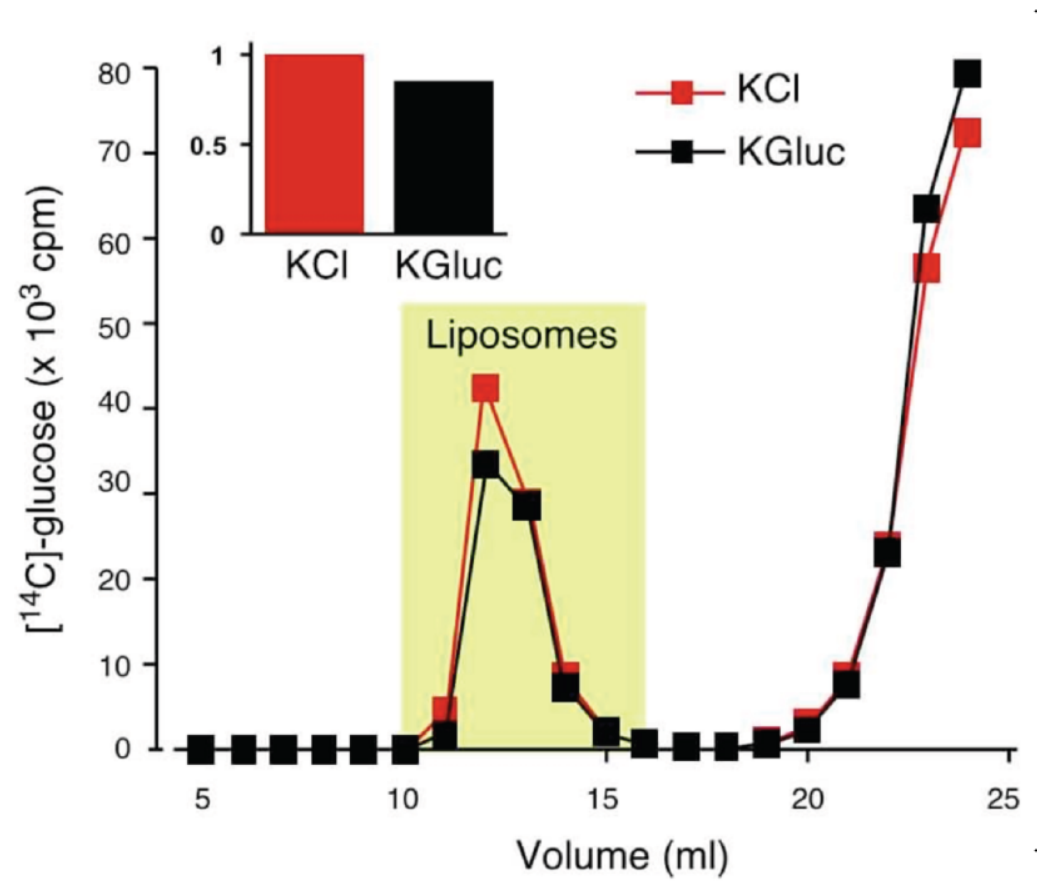

Figure $\mathbf{3 . 3 0}$

Relative volumes of liposomes formed in KGluc or $\mathrm{KCl}$ containing buffers (2)

Evaluation of relative total proteoliposome volume by measurement of trapped tracer. Proteoliposomes were formed in $\mathrm{KCl}$ (red)- or KGluc (black)- based buffers in the presence of $25 \mu \mathrm{Ci}$ $\left[{ }^{14} \mathrm{C}\right]$-glucose as a tracer. Untrapped glucose was separated from liposomes by gel filtration. The relative amount of trapped tracer in liposome fractions is directly proportional to the volume and shown in the inset. A single experiment is shown.

For this, $25 \mu \mathrm{Ci}\left[{ }^{14} \mathrm{C}\right]$-glucose were included in the liposome mixture (including $\mathrm{TF}_{0} \mathrm{~F}_{1}$ and VGLUT1 in standard concentrations) and then dialysed against $500 \mathrm{ml}$ gluconate- or $\mathrm{Cl}^{-}$based buffer overnight. The samples were then run on Sephadex-25 coulmns and $1 \mathrm{ml}$ fractions were collected and subjected to liquid scintillation. The total signal of the turbid liposome containing fractions is very similar for both of the investigated salts (Fig. 3.30). 
This result supports the data in 3.7.1. Thus, subtle differences in the volumes of the two liposome poulations are very unlikely to account for the observations made on the glutamate transport.

\subsection{Heterologous expression and purification of VGLUT isoforms and homologues}

To test if the transport characteristics observed for VGLUT1 are evolutionary conserved and if the enhancement of glutamate transport by intravesicular $\mathrm{Cl}^{-}$can be found also in

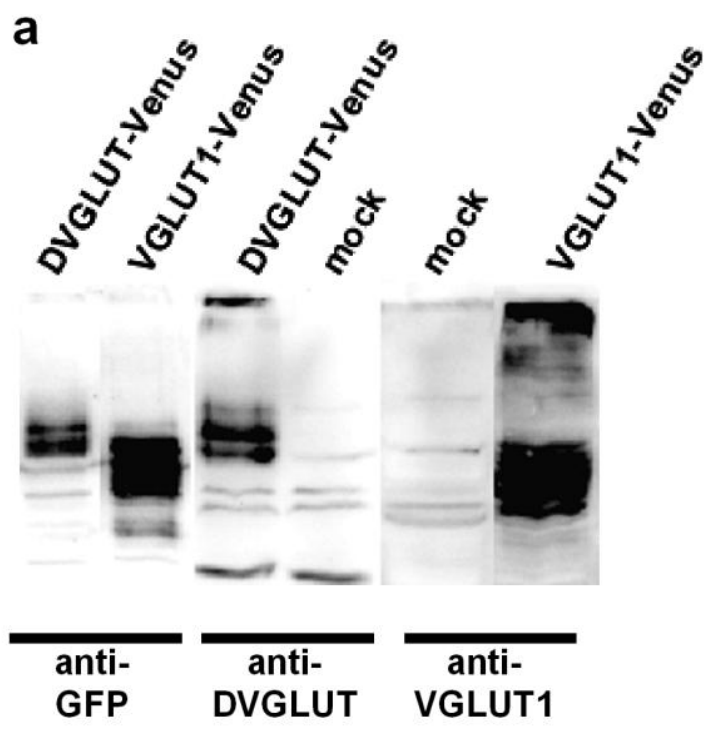

C

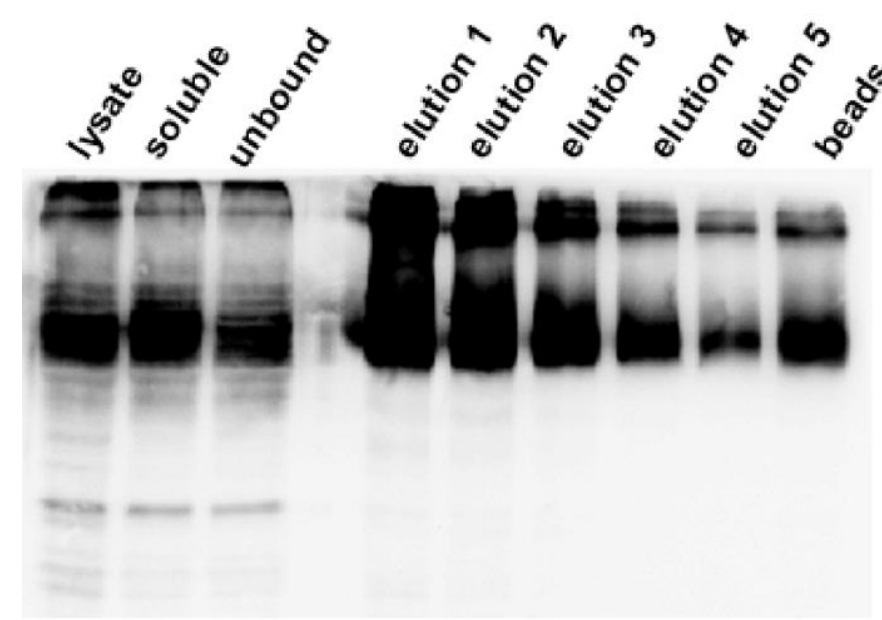

b

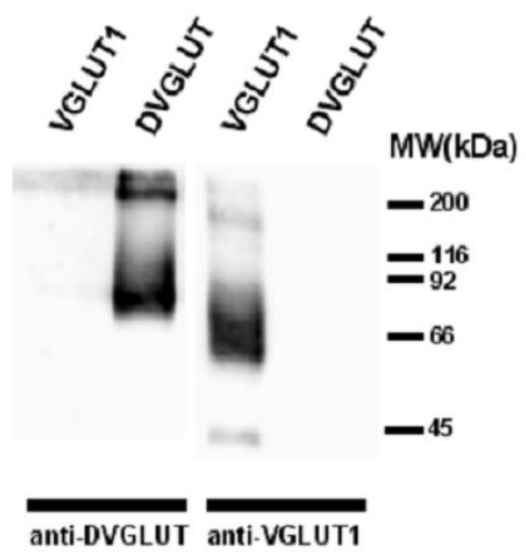

Figure 3.31

Expression and purification of DVGLUT in tsA201 cells

(a) The expression level of DVGLUT was tested by comparison of GFP-fusion proteins of DVGLUT and VGLUT1 for which the expression level is known. Soluble supernatants of transfected cells were loaded and probed with anti GFP and DVGLUT/VGLUT1 specific antibodies respectively. (b) SBP-tagged DVGLUT migrates at the expected molecular weight. (c) The purification profile of DVGLUT resembles VGLUT1 (see fig 3.13). Fractional amounts of samples loaded were the same as for VGLUT1 (Fig. 3.13). 
another major VGLUT isoform (VGLUT2), two evertebrate VGLUTs and murine VGLUT2 were subjected to expression trials in the established system by using tsA201 cells. The invertebrate VGLUTs have been biochemically inaccessible since subcellular fractions from these small animals are almost impossible to purify. Hence, they can be only investigated by genetic and physiological approaches.

\subsubsection{Expression of VGLUT from Drosophila melanogaster (DVGLUT)}

In order to get an impression of the expression level of DVGLUT in the mammalian cells, a GFP fusion protein of DVGLUT was generated (C-terminal YFP-Venus) to be able to compare the expression level directly to VGLUT1 by probing against the GFP moiety in western blots of cell lysates (analogous to the procedure used in 3.3 for $\mathrm{ClC}-3$ ). Since the results looked promising SBP-tagged DVGLUT was then expressed in a larger scale and purified in the same way as VGLUT1 (Fig. 3.31a,c). The yield was comparable to VGLUT1 with 200-400 $\mu \mathrm{g}$ from $10010 \mathrm{~cm}$ dishes and of reasonable purity. DVGLUT showed more higher-order oligomers in SDS-PAGE than VGLUT1 (Fig.3.33). The expression and purification of DVGLUT was monitored with a specific affinity purified antibody directed against the C-terminal tail (Daniels et al., 2004).

\subsubsection{Expression of eat-4 : The Caenorhabditis elegans homologue of VGLUTs}

Eat-4, which was identified in a genetic screen of $C$. elegans played a pivotal role in the identification of mammalian VGLUTs (Avery, 1993; Dent et al., 1997). Like for DVGLUT, however, no biochemical characterisation with isolated vesicles is possible. Therefore little is known about eat-4 as a glutamate transporter. Since the expression of DVGLUT was straightforward in tsA201 cells the the SBP-tag and a myc-tag (3'- end of the SBP-tag) was N-terminally fused to the eat-4 gene using overlapping PCR primers. The myc-tag was necessary to detect the protein because no specific antibody was available. First it was tested if the purification in larger scale would be promising by comparing an N-terminally myc-tagged VGLUT1 with the myc-tagged eat-4. Heterologous lysates expressing the two proteins showed that both transporters express similarly (Fig. 3.32) and the production was scaled up to 50 dishes. Eat-4, like VGLUT1 

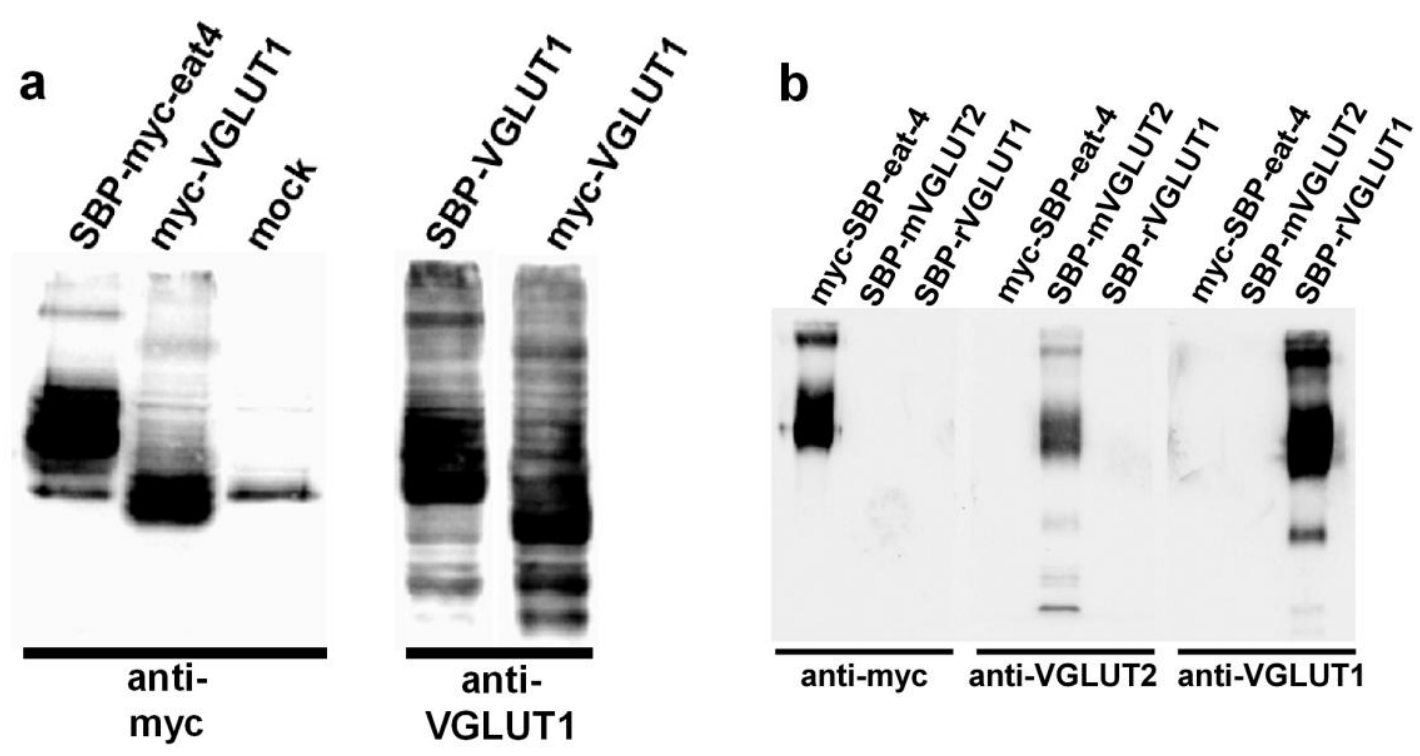

\section{Figure 3.32}

Expression of eat-4 and mVGLUT2 in tsA201 cells

(a) The expression level of eat- 4 was evaluated by the integrated myc-tag with myctagged VGLUT1. Eat-4 expresses with the expected molecular weight at levels comparable levels to VGLUT1. The expression of myc-tagged VGLUT1 is almost the same as SBP-tagged VGLUT1 (right panel).Soluble supernatants of transfected lysed cells were loaded and probed with a monoclonal anti-myc antibody or specific VGLUT1 antibodies. (b) SBP-tagged eat-4 and mVGLUT2 were expressed in tsA201 cells. Anti-myc, anti-VGLUT1 and anti-VGLUT2 specifically recognize the corresponding isoform

and DVGLUT could be purified in the same way and was of good purity (Fig. 3.32). The total yield was probably a bit higher than for VGLUT1.

\subsubsection{Expression of murine VGLUT2}

Murine VGLUT2 was fused N-terminally to the SBP-tag and expressed like VGLUT1

(Fig. 3.32). The yield and purity was almost identical the VGLUT1 isoform (Fig. 3.33).

\subsection{Characterisation of glutamate transport by DVGLUT, eat-4 and VGLUT2}

To characterize the transport of the VGLUT1 homologues some key parameters were chosen since it was not feasible to express all proteins in the same amounts that would be 
a
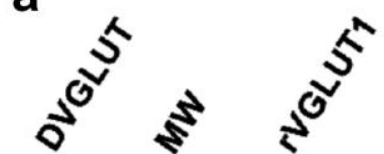

b

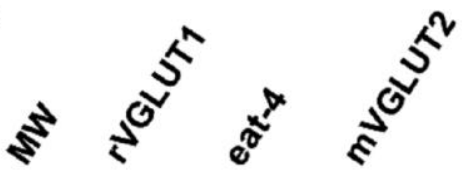

MW (kDa)
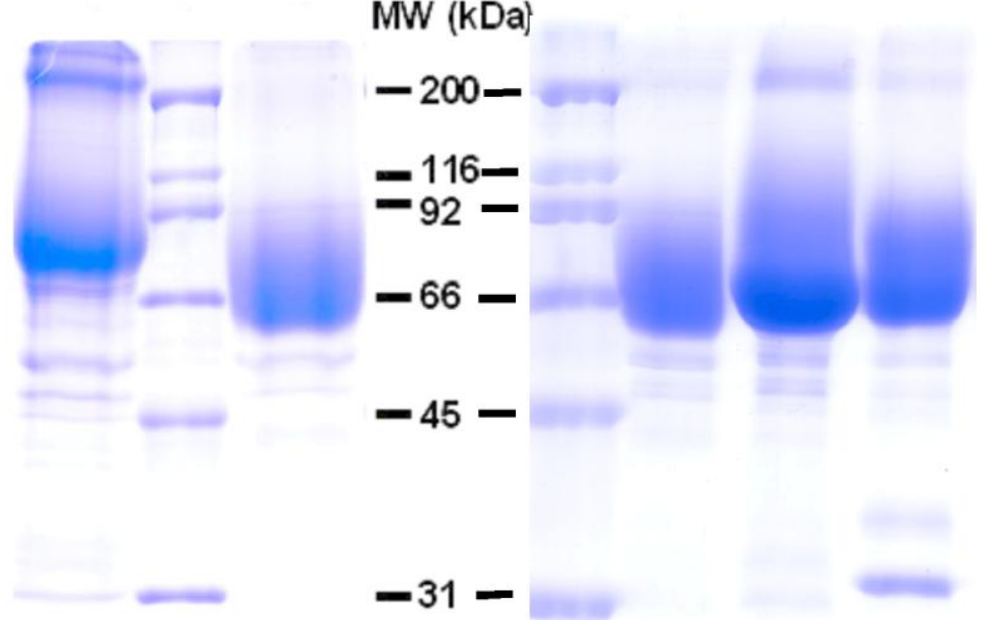

Figure 3.33

Purified isoforms of VGLUT1

(a,b) Purified VGLUTs (from

$5010 \mathrm{~cm}$ dishes each and concentrated to $300 \mu \mathrm{l})$ were loaded (12 $\mu \mathrm{l}$ each) on $10 \%$ SDS gels and stained with Coomassie blue. DVGLUT (a), eat-4 (b) and mVGLUT2 (b) are all well expressed and of comparable purity to VGLUT1. The yield of eat-4 was exceeding VGLUT1. DVGLUT shows more aggregates in SDS-PAGE.

necessary to characterize the transporters with the same depth as VGLUT1. First, it was important to see if the dependence on the internal anion is preserved among the isoforms. Second, the activation by extravesicular $\mathrm{Cl}^{-}$was analyzed by measuring the uptake in the presence of low millimolar concentrations of $\mathrm{Cl}^{-}$or in the absence of $\mathrm{Cl}^{-}$. These two conditions were also measured in the presence of Nigericin. In this way some major conclusions about the energy dependence could be drawn from relatively few experiments, hence saving protein samples. DVGLUT was characterised more detailed by measuring uptake also in the presence of various external $\mathrm{Cl}^{-}$concentrations (see Fig 3.25) because the neuromuscular junction of Drosophila is a widely used synapse in electrophysiological studies and therefore more amenable to extensive investigations. For this common model organism more information on the glutamate loading mechanism of SVs would be most beneficial. 


\subsubsection{DVGLUT transports glutamate but no aspartate}

In a first approach the specificity and ATP-dependence of the glutamate uptake by reconstituted DVGLUT was tested along with the internal anion. The uptake was measured under standard conditions (see 2.7) with $4 \mathrm{mM}$ extravesicular $\mathrm{Cl}^{-}$for $\mathrm{Cl}^{-}$-and gluconateloaded liposomes and compared to the transport in the absence of ATP and/or the presence of $10 \mathrm{mM}$ aspartate. Aspartate was not recognized as a substrate,

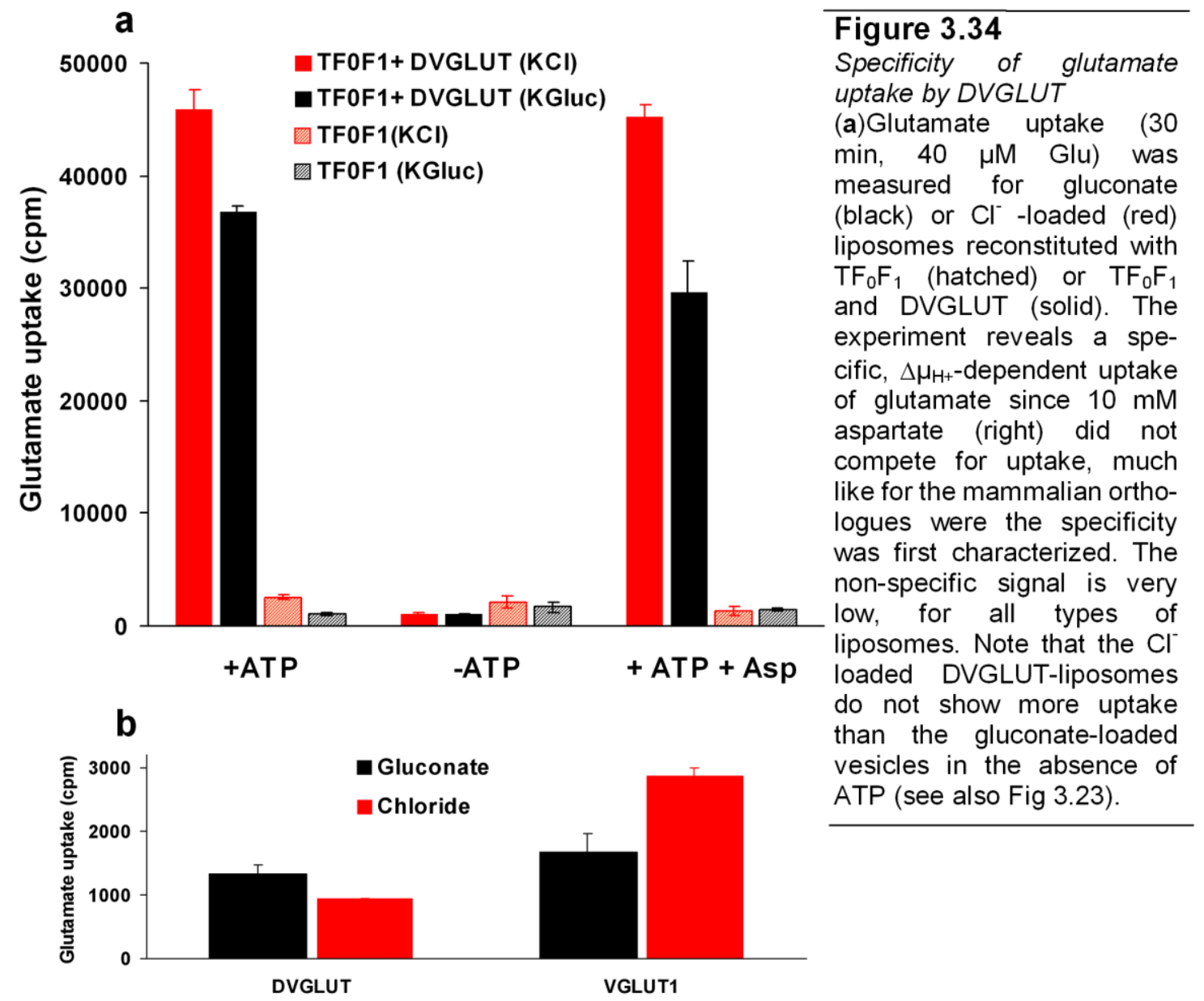

showing that the specificity for glutamate is preserved also in this isoform like for all mammalian VGLUTs (Bellocchio et al., 2000; Fremeau et al., 2002; Fremeau et al., 2001) (Fig. 3.34a). The uptake appeared to be only slightly influenced by the intravesicular anion under these conditions and was strictly dependend on the activity of the proton pump. The glutamate uptake of $\mathrm{Cl}^{-}$loaded DVGLUT-liposomes was lower in the absence of ATP 
compared to $\mathrm{Cl}^{-}$loaded VGLUT1 liposomes were some uptake occurred (Fig. 3.34b and see also Fig. 3.23b). However, the signal is relatively low, making it difficult to draw conclusions with certainty.

\subsubsection{DVGLUT shows a biphasic dependence on extravesicular $\mathrm{Cl}^{-}$and only minimal enhancement by intravesicular $\mathrm{Cl}^{-}$}

To test if the biphasic dependence, characteristic for mammalian VGLUTs is preserved in DVGLUT, a hitherto unknown parameter, the uptake of glutamate was tested under different extravesicular $\mathrm{Cl}^{-}$concentrations (Fig. 3.35). It is evident that the biphasic response is also present in DVGLUT, such that low millimolar $\mathrm{Cl}^{-}$concentrations enhance the glutamate translocation. Interestingly, however, the uptake is not strongly enhanced by intravesicular $\mathrm{Cl}^{-}$. This is most prominent in the absence of extravesicular $\mathrm{Cl}^{-}$and stands in strong contrast to VGLUT1 (Fig. 3.35 red and golden traces).

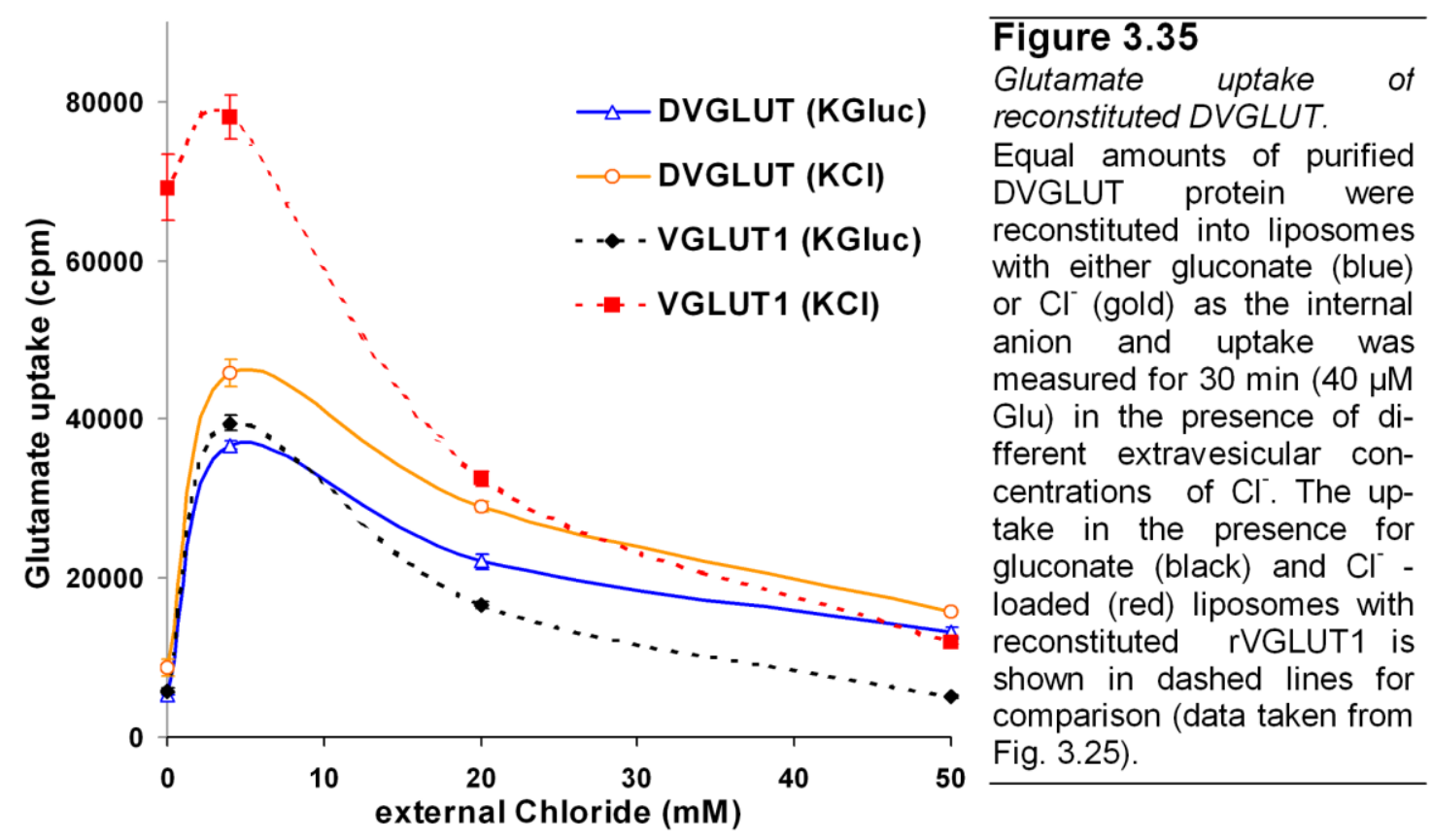




\subsubsection{The glutamate translocation by DVGLUT and eat-4 depends largely on $\Delta \mathrm{pH}$}

To gain some insight into the energy dependence of the evertebrate iosoforms, DVGLUT and eat-4 were reconstituted along with VGLUT2 and VGLUT1 and uptake was measured in the presence or absence of Nigericin at $0 \mathrm{mM}$ and $4 \mathrm{mM}$ external $\mathrm{Cl}^{-}$respectively. This was done for both gluconate- and $\mathrm{Cl}^{-}$-loaded liposomes (Fig. 3.36). The data shows that the glutamate transport by DVGLUT is only slightly enhanced in the presence of luminal $\mathrm{Cl}^{-}$ions when external $\mathrm{Cl}^{-}$is missing. Furthermore, the transport is driven mainly by $\Delta \mathrm{pH}$, since Nigericin almost abolishes the uptake in the presence of extravesicular $\mathrm{Cl}^{-}$ independent of the internal anion. Thus DVGLUT apparently is not capable to operate as a $\mathrm{Cl}^{-} /$glutamate exchanger but instead transports glutamate most likely by exchange with protons. The $\Delta \mathrm{pH}$ driven component of glutamate uptake is also present in VGLUT1 and overlaps with the $\Delta \psi$-driven antiport when luminal $\mathrm{Cl}^{-}$ions are present. The reduction in the transport of glutamate by nigericin application is therefore not as strong as for DVGLUT. The difference in transport by DVGLUT is most obvious in the absence of external $\mathrm{Cl}^{-}$where mammalian VGLUTs transport efficiently when antiport with $\mathrm{Cl}^{-}$is possible. Eat-4 shows no enhancement by luminal $\mathrm{Cl}^{-}$and apparently is even inhibited by high luminal $\mathrm{Cl}^{-}$concentrations. This is a very surprising finding and currently difficult to explain. Eat-4 also shows the most pronounced dependence on $\Delta \mathrm{pH}$ as Nigericin has the strongest effect - almost abolishing the transport. The C. elegans homologue is like other VGLUTs activated by low millimolar extravesicular $\mathrm{Cl}^{-}$concentrations and, judging from the effect of nigericin, by establishment of a $\Delta \mathrm{pH}$ in the lumen. However, further investigations are needed to get a clearer picture of glutamate translocation by eat-4. For instance, it could be that even higher extravesicular $\mathrm{Cl}^{-}$concentrations enhance the glutamate uptake further. 
a

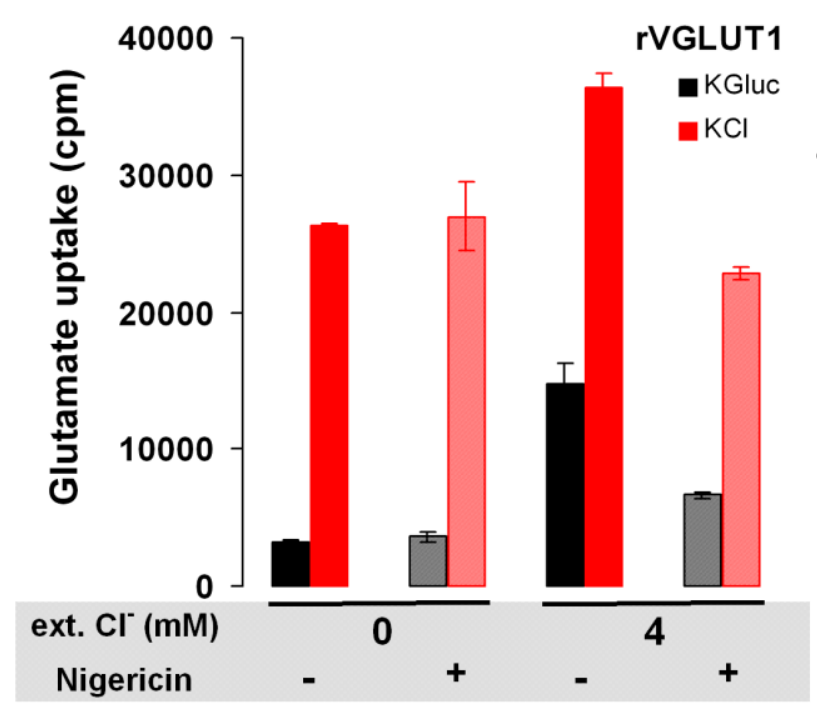

b

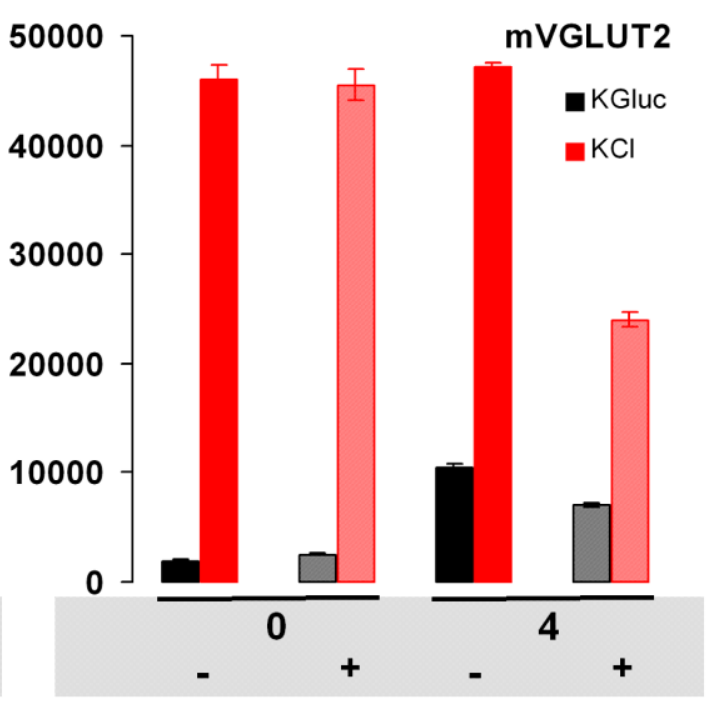

C

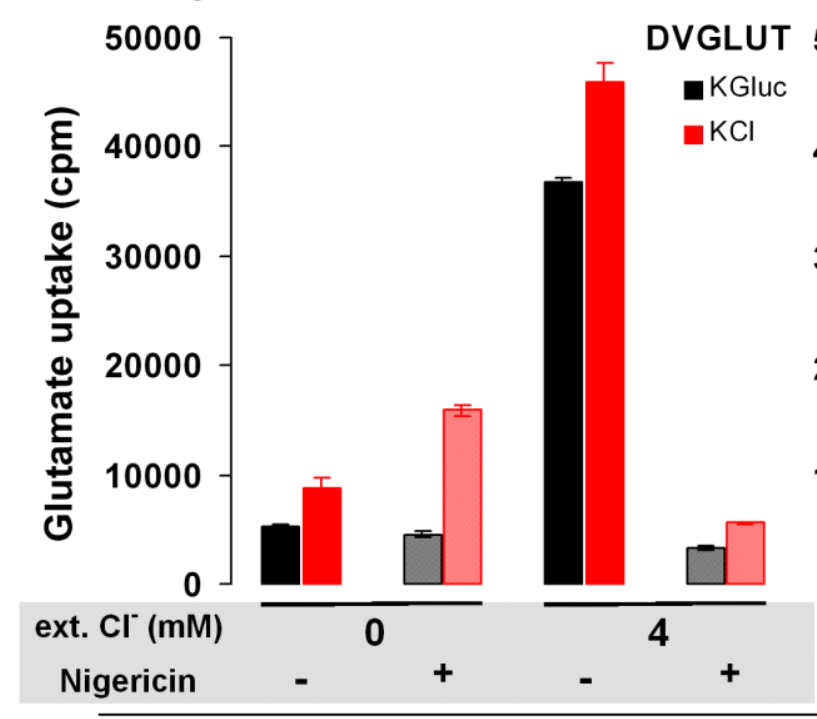

d

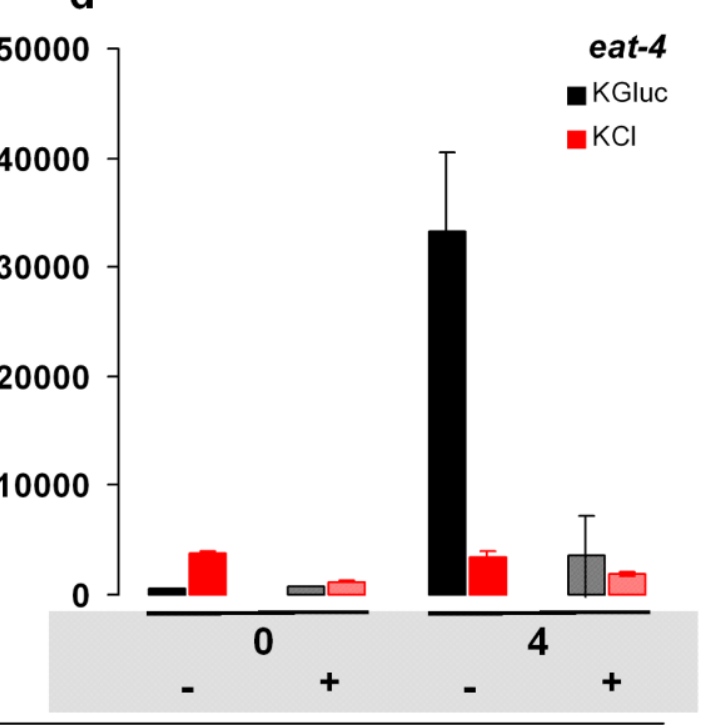

Figure 3.36

Comparison of the $\mathrm{Glu} / \mathrm{Cl}$ antiport activity and dependence on $\Delta \psi$ and $\Delta \mathrm{pH}$ for four different vesicular glutamate transporters

(a-d) To evaluate the evolutionary conservation of the facilitation of glutamate transport by intraluminal $\mathrm{Cl}^{-}$, recombinant vertebrate and evertebrate VGLUTs were reconstituted into liposomes filled with either KGluc (black) or $\mathrm{KCl}$ (red) and uptake was measured for $30 \mathrm{~min}$. In order to address the energy dependence for mouse VGLUT2 (b), DVGLUT(c) and the $C$. elegans orthologue eat-4 (d) compared to VGLUT1 (a), the uptake was measured in the presence or absence of $4 \mathrm{mM} \mathrm{Cl}^{-}$and with (hatched bars) or without Nigericin (solid bars). The data clearly indicates that the two major VGLUT-isoforms from rodents are greatly enhanced by luminal $\mathrm{Cl}^{-}$and operate to a large extent with $\Delta \psi$ as energy source $(a, b)$. In contrast, the evertebrate VGLUTS, appear to operate largely by using $\Delta \mathrm{pH}$ since Nigericin is largely diminishing transport in the presence of external $\mathrm{Cl}^{-}(\mathrm{c}, \mathrm{d})$. Luminal $\mathrm{Cl}^{-}$apparently leads to no big enhancement for the uptake of glutamate by DVGLUT and eat-4. Interestingly eat-4 seems to be efficiently inhibited by high luminal concentrations of $\mathrm{Cl}^{-}(100 \mathrm{mM})(\mathrm{d})$. 


\subsubsection{Murine VGLUT2 shows the same energy dependence pattern as VGLUT1 and exhibits enhanced transport by intravesicluar $\mathrm{Cl}^{-}$}

VGLUT2 shows a very similar enhancement by intravesicular $\mathrm{Cl}^{-}$to VGLUT1. In the presence of luminal $\mathrm{Cl}^{-}$, extravesicular $\mathrm{Cl}^{-}$is not required to drive glutamate uptake suggesting a similar $\mathrm{Cl}^{-}$/glutamate antiport mechanism as in VGLUT1 (Fig. 3.36). The uptake appears to be driven even stronger by intravesicular $\mathrm{Cl}^{-}$. The energy dependence resembles largely the one observed for VGLUT1 and indicates that mammalian VGLUTs are different in their transport characteristics from invertebrate VGLUTs. Since the synapses where VGLUTs operate are serving different functions in invertebrates and vertebrates, neuromuscular junctions and CNS respectively, the different demands in the specific physiological function of those synapses may reflect the diversity in transport mechanisms. Again, it is not possible yet to conclude certainly from the few data available, however, the observations are relatively clear and could help to understand the details of the glutamate translocation in these isoforms.

\subsubsection{DVGLUT exhibits a $\mathrm{Cl}^{-}$shunting activity in liposome acidification}

Interestingly, DVGLUT is still enhanced by extravesicular $\mathrm{Cl}^{-}$in a very similar manner as the mammalian VGLUTs, although intravesicular $\mathrm{Cl}^{-}$does not seem to have an effect on the transport. This immediately raises the question whether $\mathrm{Cl}^{-}$can permeate through DVGLUT and could help to understand if the influx and efflux of $\mathrm{Cl}^{-}$in VGLUT1 are mediated by the same mechanism. Therefore DVGLUT was assayed by AO quenching in the presence of $100 \mathrm{mM}$ extravesicular $\mathrm{Cl}^{-}$(Fig. 3.37). Indeed, $\mathrm{Cl}^{-}$permeates DVGLUT, which is compatible to the effect of nigericin that reduces the glutamate uptake when 4 $\mathrm{mM}$ external $\mathrm{Cl}^{-}$is present. The data thus suggests that the entry of $\mathrm{Cl}^{-}$through VGLUTs is preserved and that the pathway or leak for this entry is different from the efflux that was observed in the mammalian VGLUTs. Since extravesicular $\mathrm{Cl}^{-}$diminishes the uptake in the presence of nigericin also at low millimolar concentrations in VGLUT1 (see fig. 3.25, red dashed traces) the site for $\mathrm{Cl}^{-}$entry could indeed be the glutamate binding site. It is conceivable that $\mathrm{Cl}^{-}$leaks through the glutamate entry site which 
a

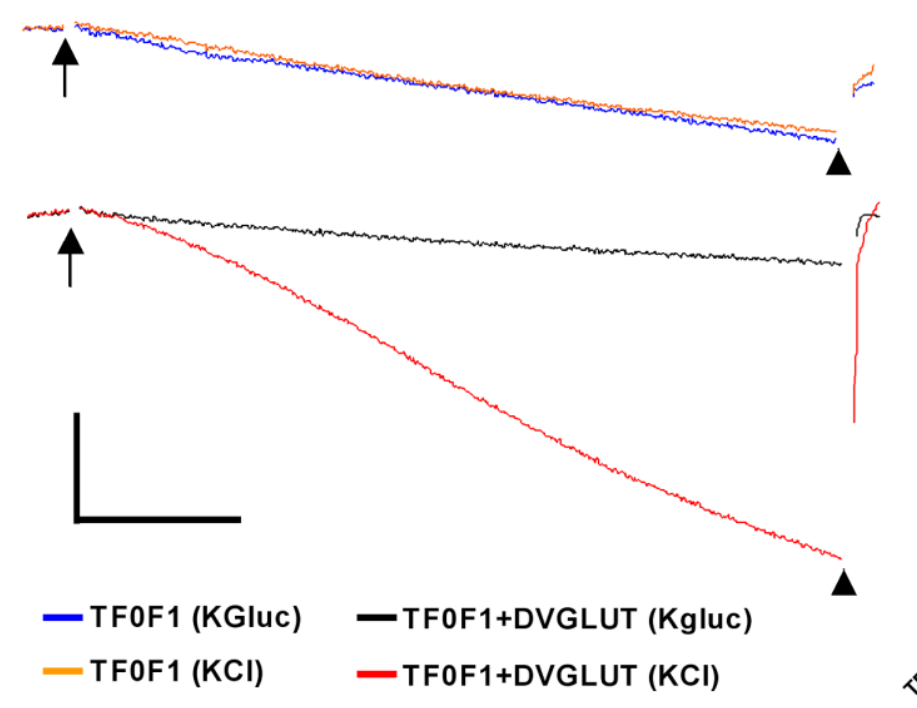

b

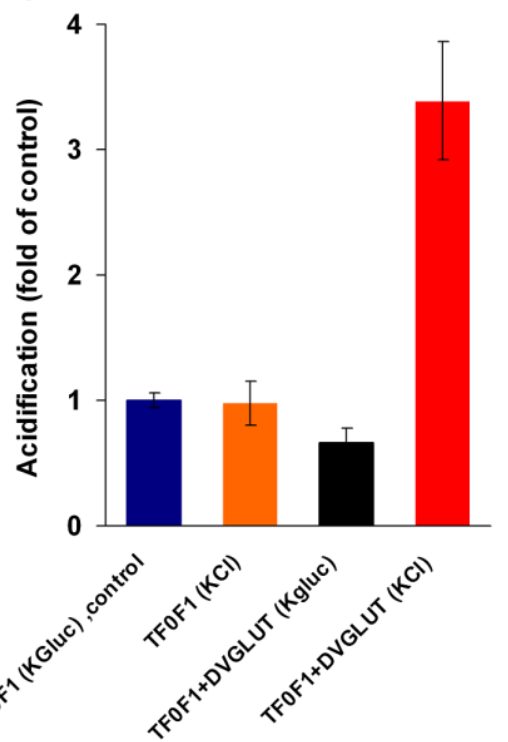

Figure 3.37

$\mathrm{Cr}$ induced acidification of DVGLUT liposomes

(a) To test if DVGLUT is capable of anion-shunting, the acidification of liposomes in the presence of $100 \mathrm{mM}$ external gluconate (black,blue) and $100 \mathrm{mM}^{2}$ external $\mathrm{Cl}^{-}$(gold,red) was tested by $\mathrm{AO}$ quenching. The upper blue and goladen traces are control liposomes bearing only $\mathrm{TF}_{0} \mathrm{~F}_{1}$. Mean values of triplicate measurements are shown. DVGLUT can support $\mathrm{Cl}^{-}$shunting. The scale bar shows $500 \mathrm{AU}$ and 120s repectively. ATP and FCCP addition are marked by an arrow and arrowhead respectively (b) Quantitative representation of the data in (a)

would also explain the competitive character of $\mathrm{Cl}^{-}$. However it is not possible to fix such a view yet since no mutational or structural information could support this view. It is nevertheless an interesting finding that can help to elucidate the transport mechanism of glutamate and the anion shunting in SVs in future investigations.

\subsection{The differences of invertebrate and vertebrate VGLUTs are useful for structure-function studies}

Invertebrate VGLUTs surprisingly did not show an enhancement of glutamate transport by luminal $\mathrm{Cl}^{-}$, although one could imagine it is a conserved feauture to achieve fast and maximal loading of SVs. While it remains currently speculative why this difference exists, it nethertheless offers a starting point for structure-function studies of VGLUTs. For instance it could help to understand how mammalian VGLUTs can achieve either $\mathrm{H}^{+}$or $\mathrm{Cl}^{-}$ antiport with glutamate. This is even more remarkable since the two ions bear an opposite charge and are unlikely translocated by the same amino acid framework in the protein. It is likely that the residues in the VGLUTs that are indispensible for the translocation of the 
substrates across the membrane and define the specific binding sites are located within transmembrane helices or at its borders and not in extended loops. In order to find the reason for the differnece in substrate translocation among the VGLUTs, it would make sense to focus on such areas and try to localize nonconserved residues. Hybrid-transporters could be generated by site directed mutagenesis and these mutants could then be tested for their energy dependence.

\subsubsection{Topology prediction of VGLUT1 using the TMHMM server}

To locate the putative transmembrane domains a topology prediction of VGLUT1 using the TMHMM server was performed (Sonnhammer et al., 1998). VGLUT1 has been proposed to bear 10-12 transmembrane regions (TMRs). The TMHMM topology prediction suggests 12 TMRs with $\mathrm{N}$ - and C-termini located at the cytoplasmic side (Fig. 3.38). The overall structure resembles the lactose permease, which is made up of two mirrored segments that are connected by a linker. Compatible with the current view of the secondary structure of VGLUTs, the termini are both located at the cytoplasmic side (Almqvist et al., 2007; Fei et al., 2007). Two TMRs , 3 and 9, are unusual compared to the other TMRs in this prediction. They also get relatively high scores for being either intracellular or extracellular and thus might be accessible from both sides. Interestingly they are also placed symmetrically to each other, supporting the idea of a gene duplication. With the TMHMM server the TMRs could be easily identified and this should be helpful to interpret an alignment among invertebrate and vertebrate VGLUTs to find hints for structural differences.

\subsubsection{Alignment of vertebrate and invertebrate VGLUTs reveals only few non- conserved residues in the transmembrane domains}

Since the difference in the energy dependence and the lack of an $\mathrm{Cl}^{-} /$glutamate antiport must bear a signature in the primary amino acid sequence of the evertebrate VGLUTs, all investigated VGLUTs of this study were aligned with the MegAlign software 


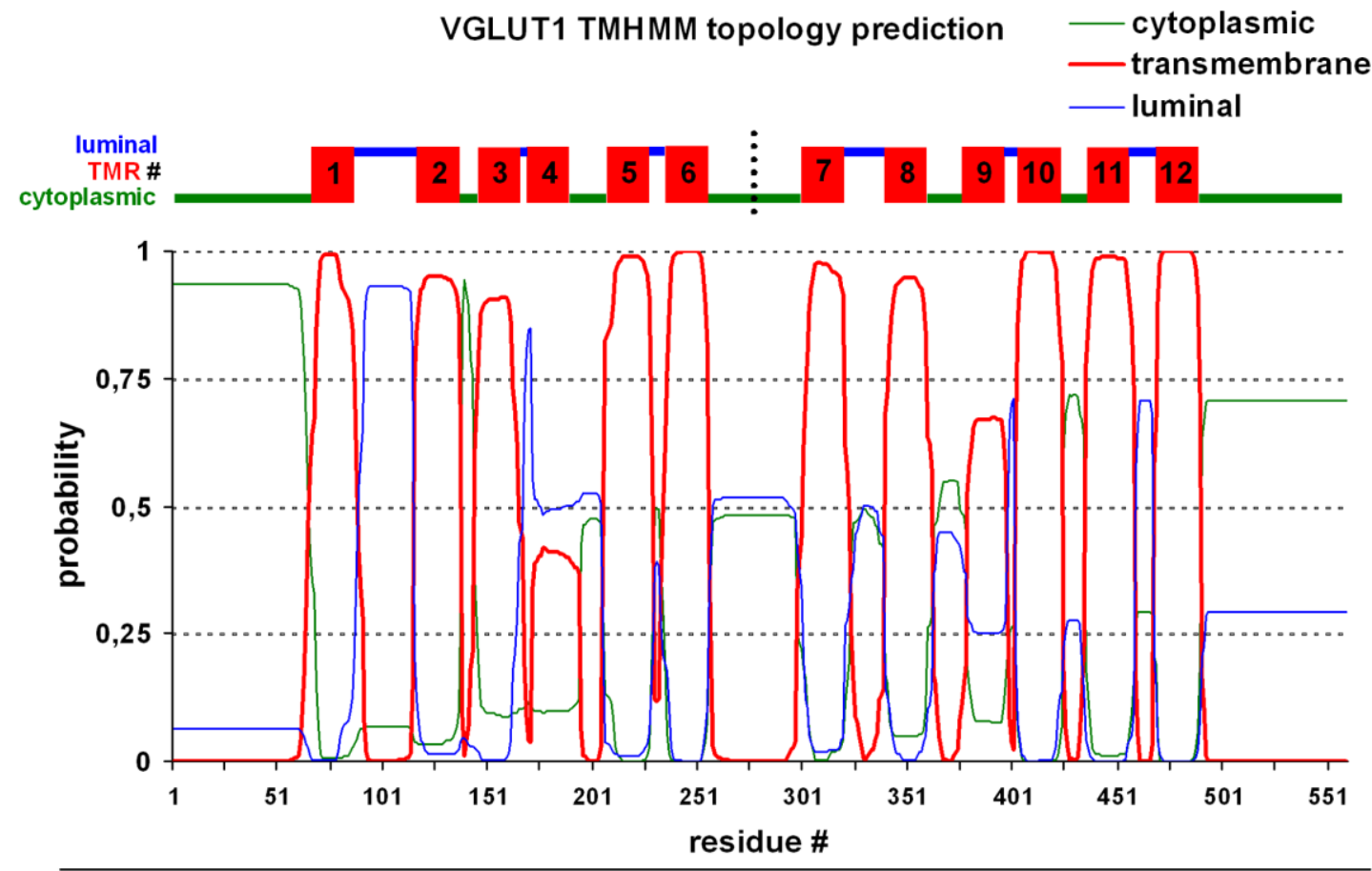

\section{Figure 3.38}

Topology prediction of VGLUT1using TMHMM

The amino acid sequence of rat VGLUT1 was subjected to the TMHMM server v. 2.0 secondary structure prediction for membrane proteins (URL: http://www.cbs.dtu.dk/services/TMHMM/ ). The output is given in probabilties for each residue to be either transmembrane (red line), cytoplasmic (green) or luminal (blue). From this a simplified model was made (on top of the figure) indicating the putative mirror plane with small asterisks.

(Lasergene) using a PAM250 matrix (Fig. 3.39). The secondary structure prediction (see 3.10.1) was also made with eat-4 and DVGLUT and resembles VGLUT1. Thus it was possible to narrow down the TMRs for all of the transporter isoforms in the alignment and look for non-conserved residues among evertebrate and vertebrate VGLUTs. In a first approach, although only one of many options to screen for, it was looked for positively charged residues (lysines or arginines) in or at TMRs that are present in mammalian VGLUTs but not in the evertebrate forms. It could be that such residues are involved in some steps (eg. the binding of $\mathrm{Cl}^{-}$at the luminal side) of a $\mathrm{Cl}^{-} /$glutamate antiport. This view is certainly very simplified, but it also serves to see how feasible it is to screen mutants and test them in a biochemical manner. Without doubt it would be much more helpful to have structural information available to locate binding sites for the substrates. 


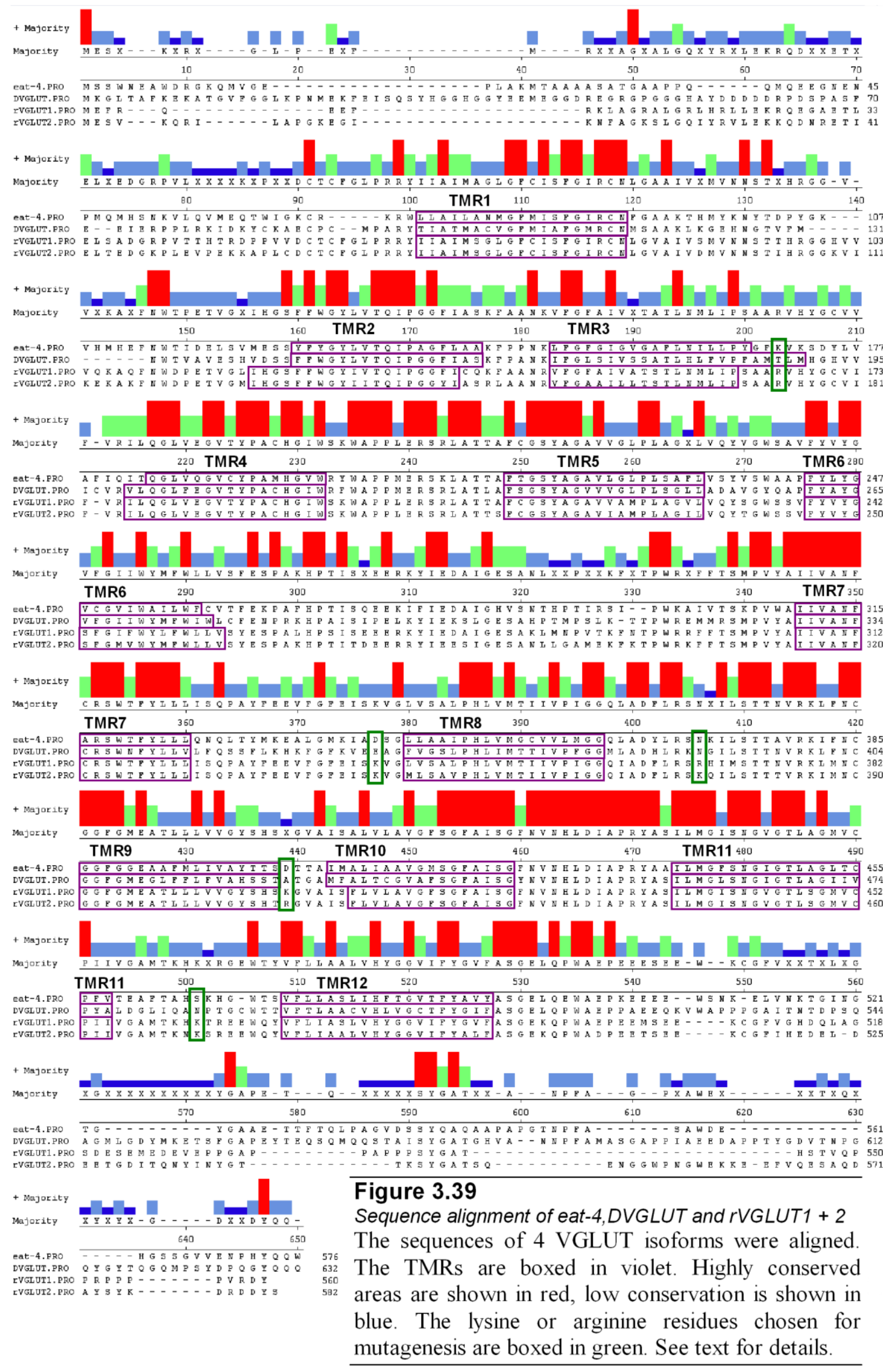


From the alignment a few non-conserved lysines and arginines were located. These residues were then mutated in VGLUT1 by site directed mutagenesis to the corresponding residue in DVGLUT.

Some of the mutations are relativley far away from TMRs and can serve as controls. Since the expression of VGLUTs is not possible in a high throughput manner, only 5 mutants were chosen to be tested: R166T, K339E, R368N, K401A and K463N.

\subsubsection{Heterologous expression of selected VGLUT1 mutant proteins}

After the mutagenesis all mutants were fully sequenced and subjected to a test expression in small scale. Soluble supernatants were subjected to SDS-PAGE,

a

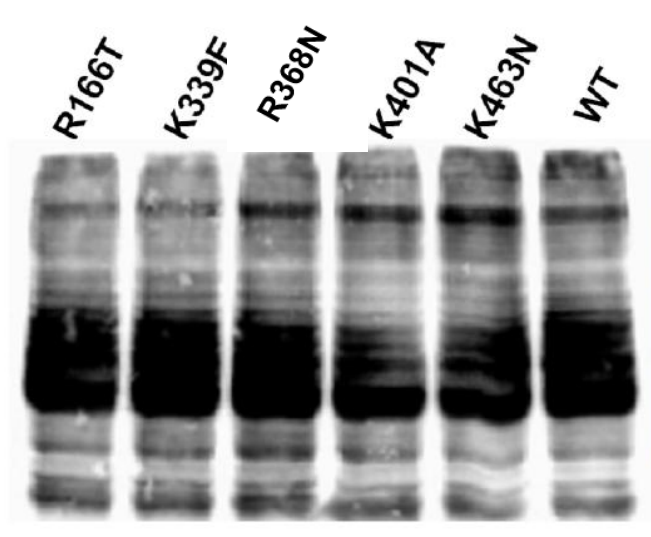

b

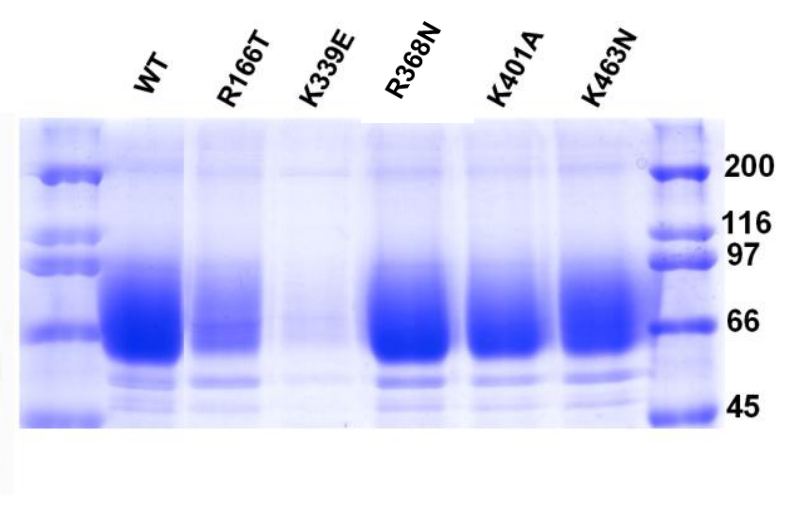

Figure 3.40

Expression and purification of 5 mutant versions of VGLUT1

(a) The expression level of 5 VGLUT1 mutants was compared with the wild type protein in tsA201 cells. Equal amounts of soluble cell lysate supernatants were probed with anti-VGLUT1 antibody. All of the introduced mutantions did not interfere with the expression level. (b) The mutant proteins were expressed at larger scale and purified like the wild type protein. Concentrated samples (same volumes) were then checked by SDS-PAGE and Coomassie staining. They all show almost the same purity. However K339E was not expressed well in this experiment and was thus not reconstituted.

transferred on blot-membranes and then probed with an anti-VGLUT1 antibody. It is apparent that all of the mutants can be expressed at reasonable levels compared to the wild type transporter (Fig 3.40a). The expression was then scaled up and the mutants were purified from $5010 \mathrm{~cm}$ dishes each. However the expression was not equally successful for all the mutants (Fig 3.40b). K339 was therefore not reconstituted. All mutants showed the same purity as the wild type protein. 


\subsubsection{Characterization of VGLUT1 mutant proteins by their energy dependence for glutamate translocation}

The purified mutants were reconstituted in liposomes with either gluconate or $\mathrm{Cl}^{-}$as the internal salt. They were then subjected to the same analysis as described for the VGLUT isoforms (see 3.9.3-3.9.4 and Fig. 3.36). If one of the mutations would suffice to change the transport characteristics of VGLUT1 towards the ones of DVGLUT it should be readily recognizable in this assay. The transport should not be enhanced by luminal $\mathrm{Cl}^{-}$and the glutamate translocation in the presence of external $\mathrm{Cl}^{-}$should be more sensitive to nigericin. However, no clear result could be obtained with the four screened mutants (Fig 3.41 and compare with fig. 3.36). Only K401A shows a tendency of lowered enhancement by intravesicular $\mathrm{Cl}^{-}$, but the uptake for $\mathrm{Cl}^{-}$loaded liposomes in the absence of external $\mathrm{Cl}^{-}$ is still substantial.

Since the knowledge of VGLUTs, and amino acid transporters in general, is still in its infancy from a structural and biochemical point of view there are probably too many unknowns so that it is not feasible to screen a big protein like VGLUT in a near-random mutagenesis approach. Unfortunately the question raised by the difference between invertebrate and vertebrate VGLUTs will also not be possible to investigate by an electrophysiological approach involving mutants. That is, because it would be necessary to observe the transport with two different intravesicular salts and thus a major hurdle for physiological experiments in vivo. Clearly, structural data would aid in revealing the transport mechanism of this interesting and important class of transporters, crucial for the function of synapses. However, it can certainly not be expected to infer the most important residues of a transporter for substrate translocation and decipher the series of binding events with only structural data. It will therefore likely be a mixture of biochemical, structural and physiological experiments that can shed light on the translocation process of VGLUTs. 
a

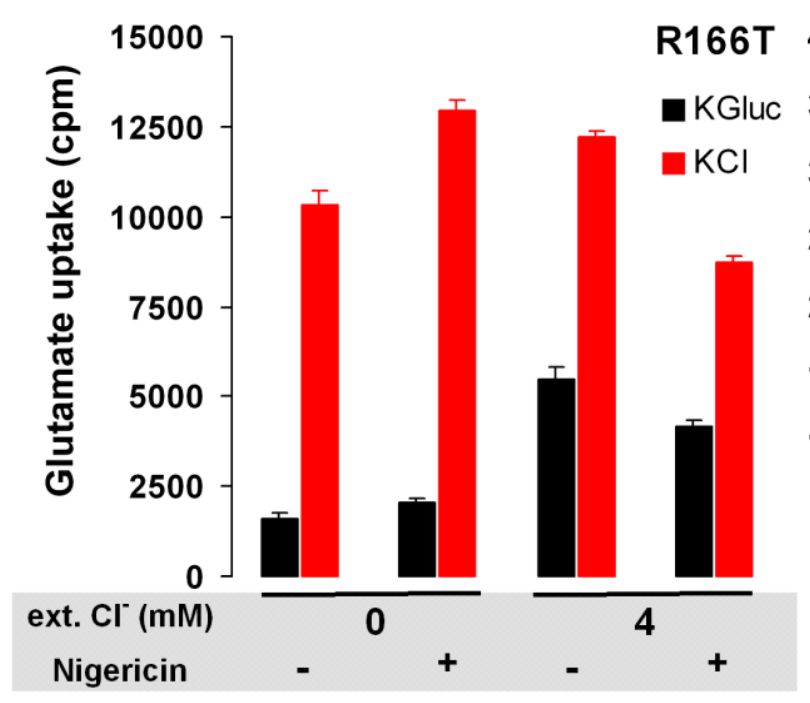

b
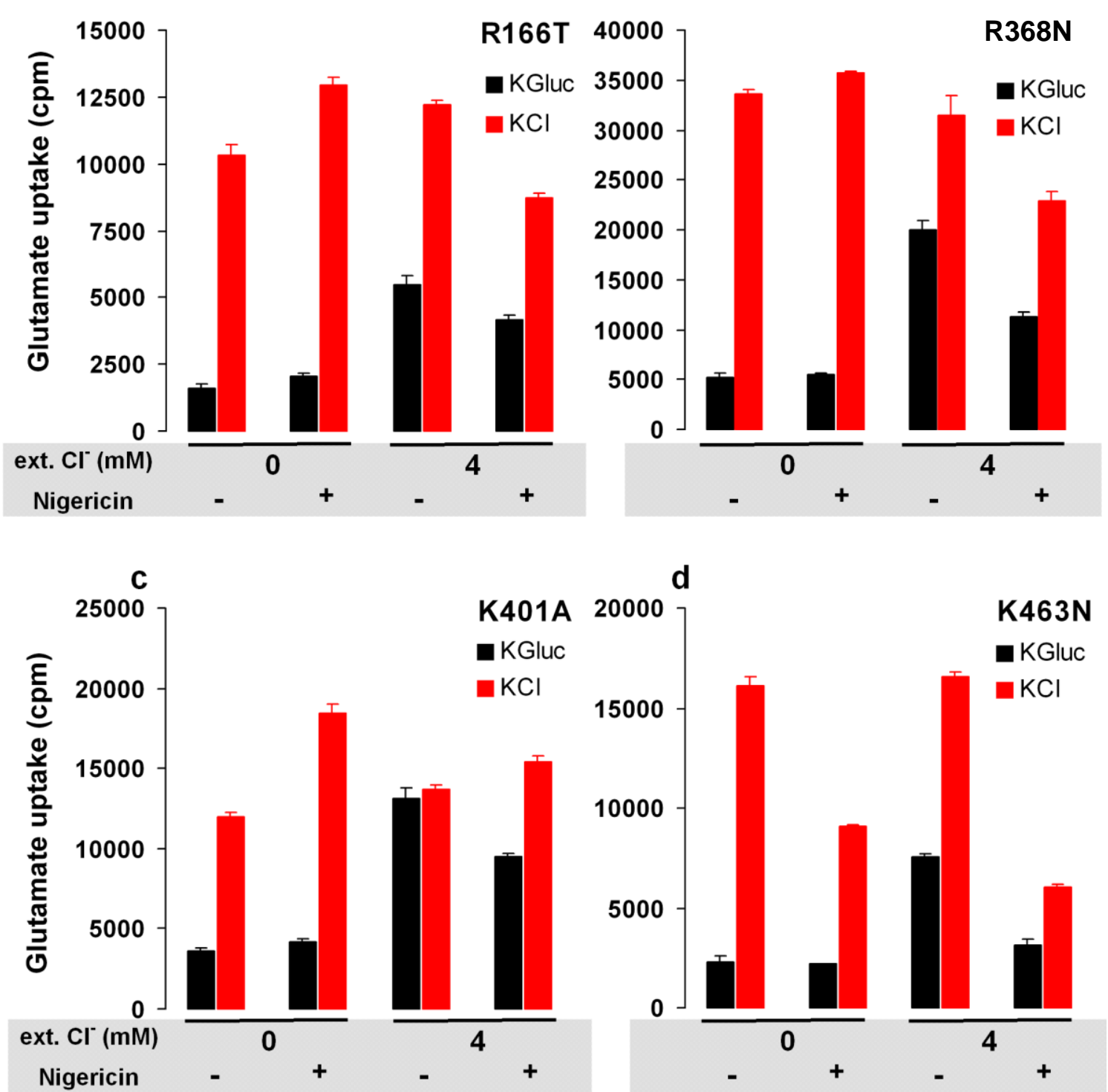

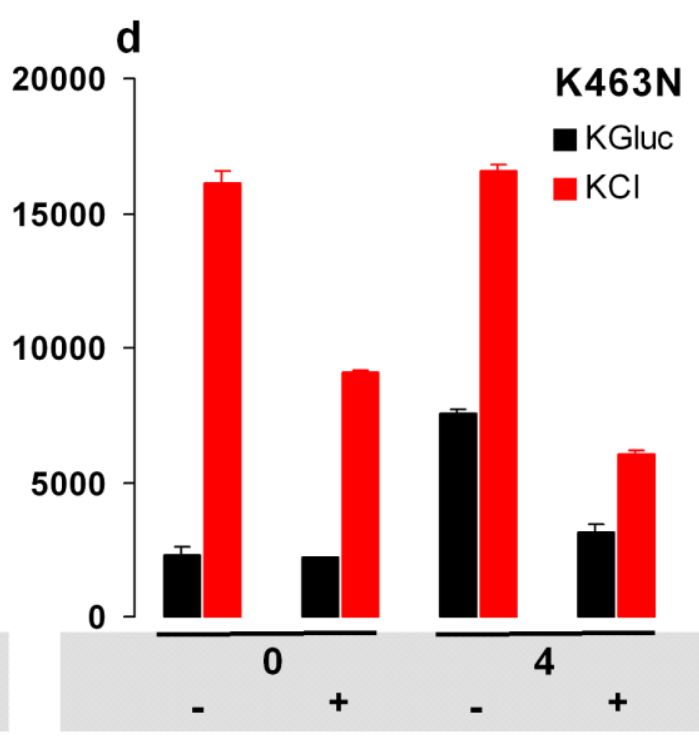

Figure 3.41

Characterisation of VGLUT1-mutant proteins by their energy dependence for glutamate translocation

(a-d) Mutant versions of VGLUT1 protein were reconstituted into $\mathrm{Cl}^{-}$- or gluconate-loaded liposomes and the transport was assayed in the presence or absence of external $\mathrm{Cl}^{-}$and/or nigericin to see if the energy dependence or antiport are disturbed or changed. The mutants were aiming at introducing transport characteristics of DVGLUT into VGLUT1 to find residues that are needed for the $\mathrm{Cl}^{-} /$glutamate antiport. None of the mutant proteins showed a clear effect, but K401A shows somewhat reduced transport when intravesicular $\mathrm{Cl}^{-}$is present compared to the transport in the presence of internal gluconate. 


\section{DISCUSSION}

\subsection{The comparison of VglutI $^{-/-}$and $\mathrm{Clcn3}^{-/-}$mouse strains clearly favors a role for VGLUT1 in $\mathrm{Cl}^{-}$shunting on SVs}

Departing from uncertainties and unresolved issues concerning the $\mathrm{Cl}^{-}$conductance on SVs, this work was aiming to shed light on the anion shunting of SVs and its influence on the glutamate loading mechanism of SVs.

The chloride conductivity of intracellular compartments is a long-known phenomenon and its investigation was promoted by the possibilty to isolate certain subcellular components like SVs to reasonable purity. These isolates could then be tested away from their cellular context and help to define some cornerstones of the luminal acidification in membraneous compartments. First, the process is ATP-dependent and requires the activity of a proton pump. Second, at least in vitro, the acidification required the presence of a permeant small anion which is $\mathrm{Cl}^{-}$. With the identification of the intracellular $\mathrm{Cl}^{-}$channels, the CLCs, a number of genes was cloned that appeared appropriate to fulfill the role of anion shunting on secretory vesicles such as SVs. This view was supported from their subcellular localisation as well as from knock-out data that showed a major impact on the endomembrane system, e.g. the trafficking defects in ClC-5 KO mice (Piwon et al., 2000), bone resorption impairment in ClC-7 KO mice (Kornak et al., 2001) and the loss of the hippocampus in ClC-3 KO mice (Stobrawa et al., 2001). The phenotype of the ClC-3 KO mice was pointing to a major role in the brain physiology and or development. 


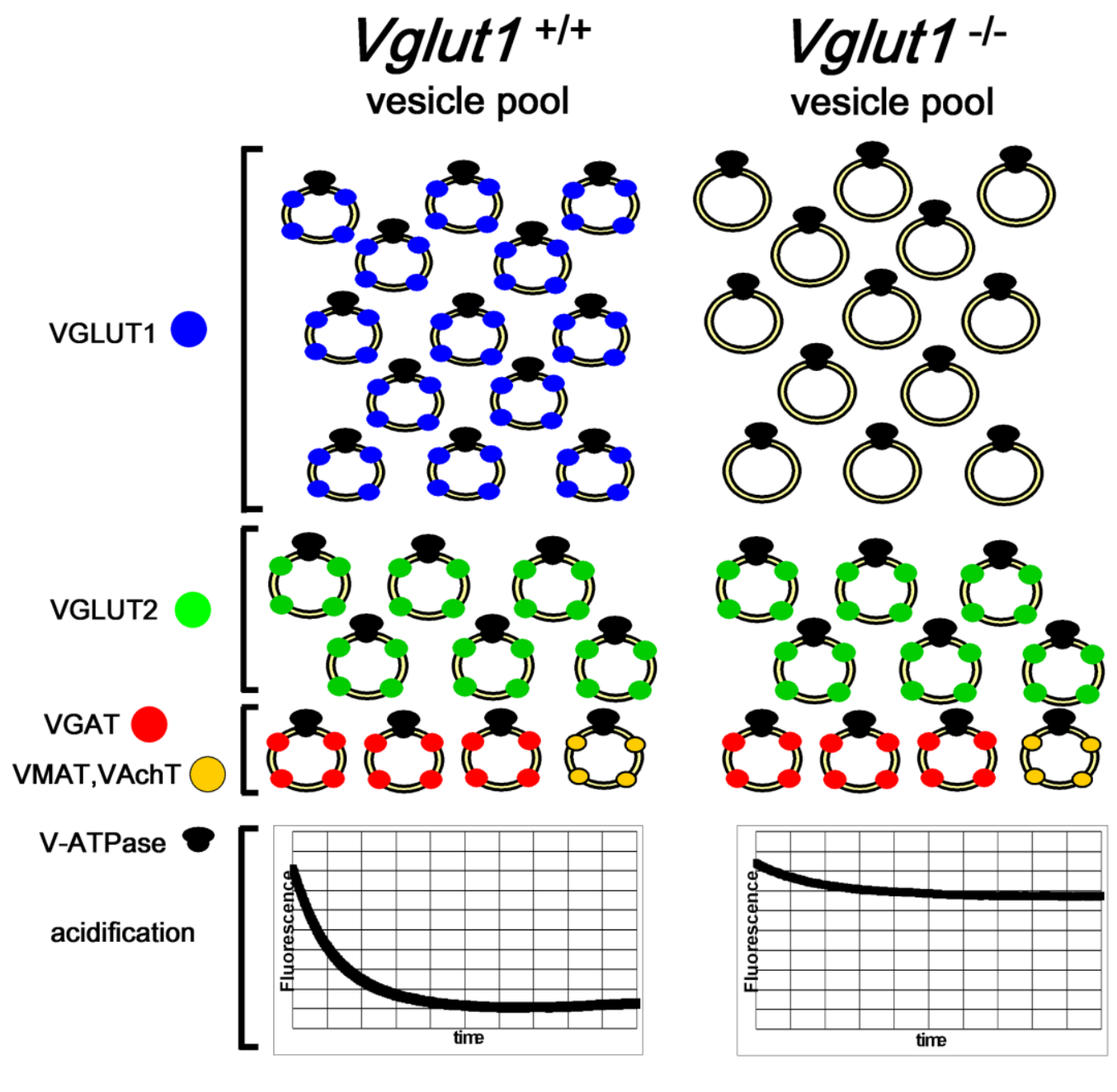

\section{Figure 4.1}

Vesicle pools of whole brain SV isolates in the VGLUT1 KO can explain the decreased acidification

Illustration of the relative fraction of SV subtypes in the total SV pool isolated from Vglut $^{+/+}$and Vglut $1^{-/-}$mice and the recorded acidification traces on the bottom. Glutamate-filled SVs comprise about $80 \%$ of SV isolates ( 60\% VGLUT1-type and $\sim 20 \%$ VGLUT2-type). GABA-filled SVs make up 18\% while VMAT and VAChT-type SVs are very rare and do not contribute to bulk biochemical measurements like acidification. Since a lot of empty vesicles without a single transporter still recycle at synaptic terminals of cultured Vglut $1^{-/}$neurons as evident by FM-dye staining / destaining, SVs isolted from Vglut $1^{-/}$mice must contain a big fraction of SVs devoid of any vesicular transporter. The decrease in acidification (which is consequently not complete since VGLUT2-SVs remain) be explained by a conductance for chloride in VGLUT1 itself.

The initial analysis of the ClC-3 KO mice was performed with tissue after the loss of the hippocampus. Severe neurodegeneration at the investigated age (after 2 months) already took place in these mice. In acidification assays, vesicles from $\mathrm{KO}$ brains acidified to a lower extent than vesicles from WT animals (Stobrawa et al., 2001). Now, when vesicles 
from very young KO mice were compared to the WT situation, no such deficits could be seen (Fig. 3.1). In the original description another interesting observation was made. When the uptake of glutamate was measured with radiolabelled substrate, the typical biphasic response for extravesicular $\mathrm{Cl}^{-}$was still observed. The glutamate uptake was lower, nevertheless it was still strongly decreased in the presence of high extravesicular $\mathrm{Cl}^{-}$ concentrations. This has always been attributed to the increase in $\Delta \mathrm{pH}$ due to a $\mathrm{Cl}^{-}$channel in SVs, however it could be hardly explained with a concept where ClC-3 is supposed to be exactly this channel. The most simple explanation for the phenotype of the $\mathrm{ClC}-3 \mathrm{KO}$ samples is that they are changed in their molecular or ultrastructural composition. One hint for this is already given by the reduction in the VGLUT1 content, probably a result of the loss of the hippocampus. If the hippocampal area is a major source for the purification of SVs, the strong expression of VGLUT1 in this brain area could be reflected in its lowered abundance in SV-samples from older $\mathrm{Clcn} 3^{-/-}$mice (Stobrawa et al., 2001) (Fig 3.1). Thus, the composition of the vesicles might be more altered than one might judge from the remaining vesicle markers. However other SV marker proteins such as VIAAT and VMAT2 are not enriched in such samples, making it difficult to find an explanation for this as the samples are normalized by their total protein content. As mentioned earlier, glutamate uptake does not only show a remarkable response to extravesicular $\mathrm{Cl}^{-}$but it was also found that VGLUT1 itself increases the conductance for $\mathrm{Cl}^{-}$when heterologously expressed in secretory granules (Bellocchio et al., 2000). With the establishment of the VGLUT1 KO it was then possible to test for this conductance in SV samples and compare it to samples from $\mathrm{Clcn}^{-/-}$mice. The analysis clearly revealed that the loss of VGLUT1 reduced the $\mathrm{Cl}^{-}$dependent acidification in SVs at the ages of three weeks (where no such effect was observed in the ClC-3 KO samples) and at the age of 8 weeks (Fig. 3.2). This are clear indications that VGLUT1 (and most likely also VGLUT2) is underlying the $\mathrm{Cl}^{-}$ conductance of a major fraction of SVs. The VGLUT1 KO possesses a lower number of SVs compared to wild type synapses as shown by electron microscopy (Fremeau et al., 2004), consequently the yield of SVs from Vglut $^{-/}$mice was also lower in this study (approximately by $30-50 \%$ of the WT). To overcome this, protein amounts were adjusted for all preparations to $2.5 \mathrm{mg} / \mathrm{ml}$ before the biochemical assays. In addition recent data showed a direct involvement of VGLUT1 in endocytic processes, where the C-terminal proline rich domain is interacting with endophilin proteins (Voglmaier et al., 2006). These 
proteins are known to be part of the endocytic machinery, hence it is also possible that the loss of VGLUT1 has influence on the SV number and recycling. Nevertheless, normal cycling of SVs has been shown in VGLUT1-KO neurons by FM1-43 staining/destaining of SVs (Wojcik et al., 2004). It is therefore believed that the SVs remain functional and bear all components to normal extent (see also figs. 3.2 and 3.4) but instead of discharging glutamate at the synaptic cleft they fire "blanks" as a result of being glutamate-free. If there exists a reasonably large number of SVs in the total pool, that are devoid of VGLUT1, the reduction of acidifcation in the VGLUT1 KO samples supports the idea of VGLUT1 being responsible for anion-shunting as schematically shown in figure 4.1. The data therefore speaks for role in anion shunting on SVs for VGLUT1, instead of ClC-3.

\subsection{Reconstitution of VGLUT1 into artificial liposomes confirms a $\mathrm{Cl}^{-}$conductance in vitro}

The expression and purification of components for the vesicular glutamate uptake has been one of the major hurdles to investigate the loading mechanism in detail. Isolated subcellular fractions are only of limited use to gain more insight into the transport characteristics. For instance it is not possible to rule out the influence of additional proteins which might be biased in their expression level by VGLUT1. Thus, it could be that such an effect is underlying the reduction in $\mathrm{Cl}^{-}$induced acidification in $\mathrm{KO}$ samples, e.g. by an accompanying unknown $\mathrm{Cl}^{-}$channel on SVs. Another obstacle is the ionic composition of intravesicular and extravesicular solutions. The ionic composition of the intravesicular solution is in principle not subject to manipulation. Recently the manipulation of the intravesicular space has been achieved in lysosomes for the study of $\mathrm{Cl}$ - transport in $\mathrm{ClC}-7$ (Graves et al., 2008), however it required a very high ionic strength and did not aim to keep all other components of the organelle intact. A clearly defined manipulation of the luminal space thus requires a solubilization / reconstitution procedure. Crude synaptic vesicles are however only partly suitable for this. The components of different vesicle types (e.g. VGLUT2, VIAAT and other transporters) would freely mix and then lead to an undefinded protein composition after the reconstitution of vesicles. This would make the interpretation of experimental results very difficult. 
The defined composition of both sides of the vesicle membrane is also necessary to interprete the effects of ionophores correctly. For example it is not possible to impose a $\mathrm{pH}$-gradient by the use of valinomycin if the intravesicular space does not contain any potassium. Instead, valinomycin would lead to an increase of $\Delta \psi$ if extravesicular potassium ions are available. This is in principle also problematic in the analysis of SVs from VGLUT1 KO mice. It would have been ideal to normalize the acidification that can be achieved by an artificial $\mathrm{Cl}^{-}$shunt or by counterion movement. However, no such $\mathrm{Cl}^{-}$ ionophore is known to the author and it is not possible to induce the movement of counterions if the intravesicular ion composition can not be set or is unknown.

Therefore it appears that the reconstitution of purified components would deliver the most reliable results. The analysis of the VGLUT1 KO strain, together with the indications from previous investigations (Bellocchio et al., 2000; Hartinger and Jahn, 1993; Wolosker et al., 1996) legitimated this challenge.

Since it was possible to create $\mathrm{TF}_{0} \mathrm{~F}_{1}$-liposomes that appear to be devoid of any major anion conductance (Figs. 3.18a) it was straightforward to test for an anion conductance in VGLUT1. A $\mathrm{Cl}^{-}$conductance could be validated a reconstituted system of minimal complexity (Fig. 3.18b-c, 3.19a). The potential of the liposomes to acidify was tested with valinomycin and showed that VGLUT1 only introduces a conductance for $\mathrm{Cl}^{-}$but did not change the properties of the vesicles (Fig. 3.19b). Furthermore it could be confirmed that the conductance is not due to a copurified contaminant since the pulldown of VGLUT1 from the liposome premix greatly reduced the signal change in the AO assay (Fig. 3.20). In addition the transporter comigrated with the $\mathrm{Cl}^{-}$conducting activity (Fig 3.21) in SEC. This supports the previous findings, although the resolution of SEC is rather limited.

Since all the available data clearly supported a conductivity for Cl- in VGLUT1, the most important question remained: What is the function of this conductance and how does it relate to the uptake of glutamate?

\subsection{The glutamate loading mechanism of SVs: The role of extra- and intra- vesicular $\mathrm{Cl}^{-}$ions.}

It was then analysed in a variety of experiments how $\mathrm{Cl}^{-}$influences the glutamate uptake. Since the conductance and the glutamate transport activity arise from the same protein, all 
further experiments can be interpreted having this in mind. Notably, a biphasic dependence of uptake on the extravesicular $\mathrm{Cl}^{-}$concentration was observed also in the reconstituted system which strongly indicates that the observations on SVs are also based on only two components, the V-ATPase and VGLUT. This makes an additional $\mathrm{Cl}^{-}$channel for VGLUT-positive vesicles obsolete, although its existence cannot be excluded with certainty (VGLUT1 / 2 positive SVs comprise $~ 80 \%$ of all SVs (Takamori et al., 2006)). However, the most important finding is represented by the strong enhancement of glutamate transport by luminal $\mathrm{Cl}^{-}$, a condition that was experimentally inaccessible prior to reconstitution of purified components. In summary, the observations allow for a series of novel conclusions about the glutamate loading mechanism of SVs:

1. VGLUT1 efficiently transports glutamate solely driven by $\Delta \Psi$, but to do so a charge compensating exchange with a luminal anion is necessary. $\mathrm{Cl}^{-}$is serving this role and the $\mathrm{Cl}^{-}$permeation pathway is provided by VGLUT1 itself (Figs. 4.2 and 4.4). If a permeable internal anion is missing, the $\Delta \Psi$-driven transport is extremely low.

2. When external $\mathrm{Cl}^{-}$is present, part of the transport will depend on $\Delta \mathrm{pH}$, hence be sensitive to NIG (Fig. 3.25 and Fig. 4.2, left panel). Again, this can be attributed to the $\mathrm{Cl}^{-}$ conductance in VGLUT1 since in the reconstituted system only VGLUT1 can provide a shunting current that would lead to an increase of $\Delta \mathrm{pH}$. Apparently, VGLUT1 can operate also as an $\mathrm{H}^{+} /$glutamate exchanger and, if no permeable anion is present on the luminal side, will transport glutamate almost entirely in this fashion, since the remaining NIG insensitive component is very small (Fig. 3.25a-b, open black circles).

3. Low millimolar concentrations of extravesicular $\mathrm{Cl}^{-}$do not activate transport by regulatory binding to VGLUT1 contrary to previous interpretations (Hartinger and Jahn, 1993; Juge et al., 2006; Winter et al., 2005). When the $\Delta \mathrm{pH}$ component is abolished by NIG, $\mathrm{Cl}^{-}$reduces uptake also at low concentrations (Fig. 3.25a-b, open red triangles). This effect is stronger when glutamate concentrations are low, pointing to the competitive character of $\mathrm{Cl}^{-}$. Consequently, high external $\mathrm{Cl}^{-}$concentrations reduce the uptake further since $\mathrm{Cl}^{-}$is competing with glutamate for $\Delta \Psi$-driven entry and/or occupies the transporter in a certain conformational state. 
Another indication for the general importance of $\Delta \Psi$ for both translocation mechanisms can be deduced from the application of VAL on $\mathrm{Cl}^{-}$loaded liposomes. This dramatically reduces the uptake (Fig. 3.26). Since it was shown here that the $\mathrm{pH}$ gradient can also serve as a driving force one might wonder why this $\mathrm{pH}$-gradient can not be utilized. The most likely explanation would be that $\Delta \Psi$ is essential to translocate glutamate also for $\mathrm{H}^{+} /$glutamate antiport. This is also supported by the low uptake of $\mathrm{Cl}^{-}$ loaded liposomes in the absence of ATP.

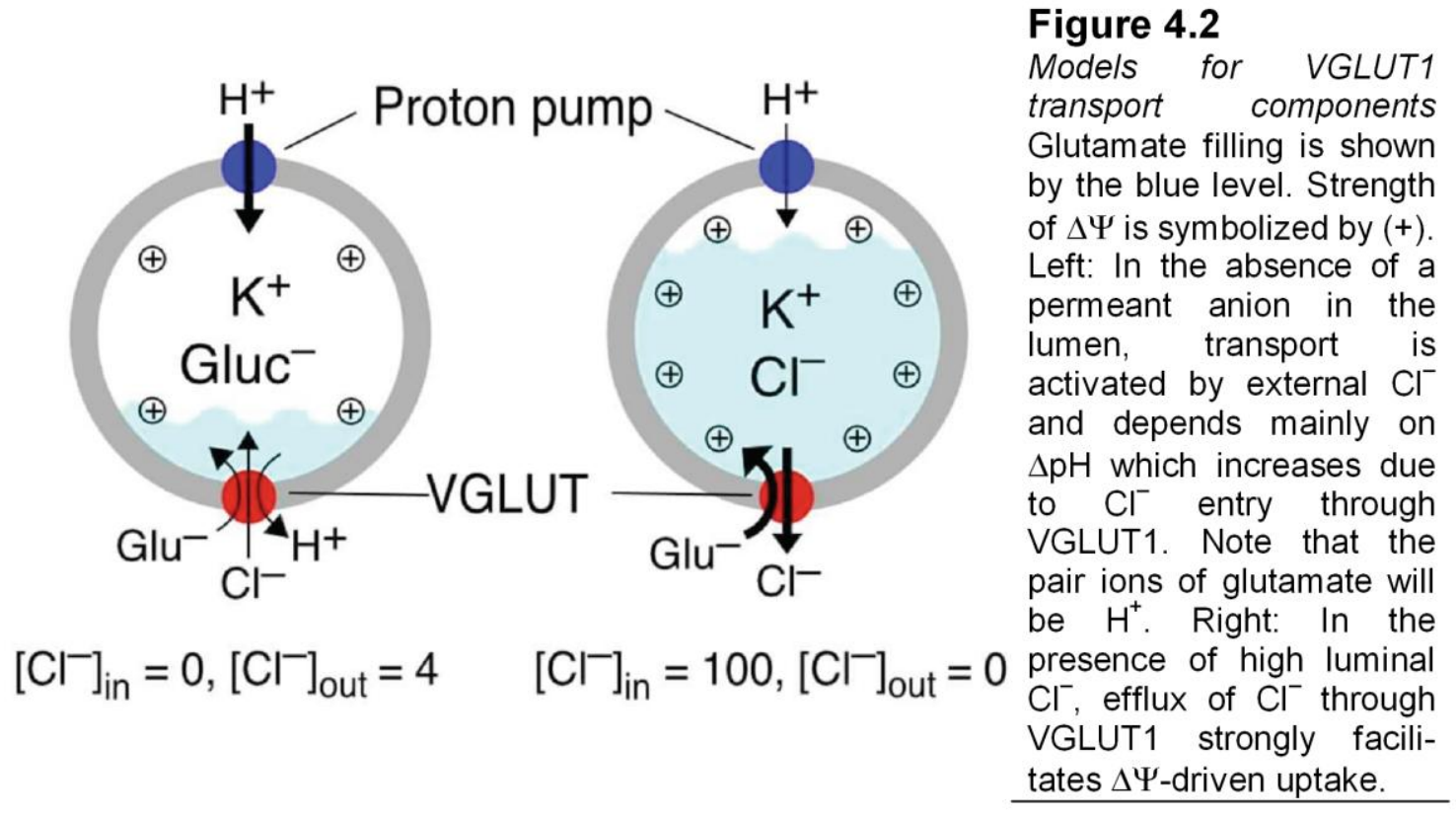

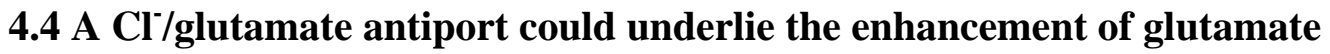 transport in $\mathrm{Cl}^{-}$loaded liposomes}

Since SVs are loaded with $\sim 100 \mathrm{mM}$ glutamate (Burger et al., 1989), it appears more likely that the bigger portion of the uptake in SVs is driven by $\Delta \Psi$, because the $\mathrm{H}^{+} /$glutamate exchange is restricted by osmotic barriers and would require swelling of SVs. Storage of glutamate as free acid in SVs would result in very low $\mathrm{pH}$ of 3-3.5, over two $\mathrm{pH}$-units lower than reported values (Miesenbock et al., 1998) and the luminal osmolality would reach values of $400-500$ mOsm which are unlikely to persist in SVs. The only way to 
evade this physical barrier is the efflux of other osmolytes, since there is no evidence for a transmitter-storing matrix in glutamate-filled SVs (Takamori et al., 2006). In a scenario where $\mathrm{Cl}^{-}$is extruded in parallel with glutamate uptake (Fig. 4.2, right panel and Fig. 4.3), the transmitter would be stored mainly as sodium glutamate, thus overcoming the drop of $\mathrm{pH}$, insolubility and osmotic imbalance as proposed earlier (Maycox et al., 1990b). It also explains why the uptake usually assayed in isolated SVs under maximal $\Delta \Psi$ is very low and resembles the uptake observed in gluconate-loaded liposomes. Isolated SVs must have lost most of the luminal $\mathrm{Cl}^{-}$during the glutamate loading process in the synapse (Fig. 4.3). Indications for the exchange of $\mathrm{Cl}^{-}$and glutamate have been obtained also with native vesicles, which led to the proposal of an $\mathrm{Cl}^{-}$/ glutamate antiporter model (Wolosker et al., 1996). When SVs were preloaded with glutamate, application of $\mathrm{Cl}^{-}$in relatively high concentrations to the assay medium induced efflux of glutamate if $\Delta \mathrm{pH}$ was clamped. The data presented herein are largely compatible with this model. However, VGLUT1 translocates $\mathrm{Cl}^{-}$also in the absence of glutamate and $\Delta \mathrm{pH}$ can also be utilized for the uptake making the translocation more complex than assumed by Wolosker et. al.. Currently it is not possible to clearly distinguish whether influx and efflux of $\mathrm{Cl}^{-}$are mediated by the same mechanism within VGLUT1.

\subsection{Implications of the $\mathrm{Cl}^{-} /$glutamate antiport activity for the loading of glutamate in the synapse}

Some recent experiments were aiming to influence the glutamate content of SVs by raising the cytosolic $\mathrm{Cl}^{-}$concentration above normal levels (Price and Trussell, 2006) since this could be predicted by the long-known biphasic dependence on extravesicular $\mathrm{Cl}^{-}$(Naito and Ueda, 1985). However, based on the data in this work one would expect a net influx of $\mathrm{HCl}$ into SVs instead of a glutamate efflux from already charged vesicles. Most SVs in the synaptic terminal are already filled with glutamate, the attenuating effect of $\mathrm{Cl}^{-}$on glutamate loading is however measured during loading. Alternatively, the efflux of glutamate from already charged vesicles upon exposure of high concetrations of $\mathrm{Cl}^{-}$could be expected from an experiment of Wolosker et. al (Wolosker et al., 1996). However there is an important between the approaches of Wolosker et. al and Price et. al., since the 


\begin{tabular}{lrrc}
\hline$\left[\mathrm{Cl}^{-}\right](\mathrm{mM})$ & 130 & $10^{\star}$ \\
\hline$\left[\mathrm{Glu}^{-}\right](\mathrm{mM})$ & 0 & $\sim 100$ \\
\hline $\mathrm{pH}$ & 7.3 & $\sim 5.5$ \\
\hline Osm (mOsm) & 320 & & $320^{\star}$ \\
\hline
\end{tabular}

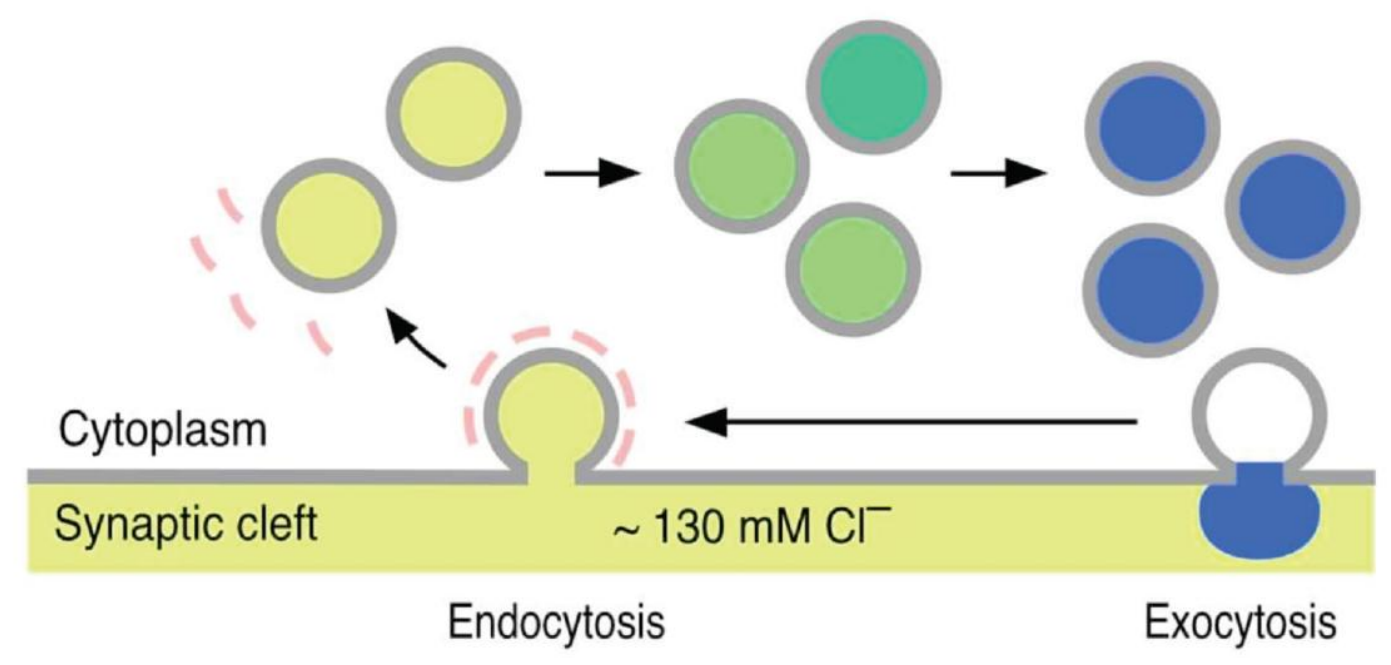

\section{Figure 4.3}

Illustration of glutamate loading at the nerve terminal during recycling of SVs

Just after endocytosis, SVs contain $130 \mathrm{mM} \mathrm{Cl}^{-}$engulfed from the extracellular space which gradually decreases in the course of glutamate loading. The luminal $\mathrm{pH}$ is decreasing as well and the osmolality would remain relatively stable during glutamate loading (see main text for explanations). $\left({ }^{*}\right)$ This value has not been measured experimentally and represents an estimate based on the transport model proposed herein.

investigations on isolated SVs were conducted in the presence of a $\mathrm{Ca}^{2+} / 2 \mathrm{H}^{+}$ionophore that clamps $\Delta \mathrm{pH}$. High concentrations of $\mathrm{Cl}^{-}$in the terminal would therefore dissipate $\Delta \psi$, and not lead to a glutamate/ $\mathrm{Cl}^{-}$counter transport. Thus, this finding is compatible with the data presented herein. Future investigations might resolve some of these issues in more detail but must also confirm turnover of SVs to see changes in the dynamics of vesicle loading.

VGLUTs have been proposed to play a role in the determination and regulation of quantal size (Edwards, 2007; Takamori, 2006). This has been addressed mainly to their copy number (Wojcik et al., 2004) or changes in vesicle size (Daniels et al., 2004), and critically depends on the filling model applied for SVs (Edwards, 2007) (see 1.3.4). The transport mode described herein is therefore an important factor to consider in this context. Although here, no experimental evidence is provided for it, the $\mathrm{Cl}^{-}$content of freshly endocytosed 
SVs would be a major determinant for the total glutamate load. If the extracellular $\mathrm{Cl}^{-}$ concentration is the limiting factor, the size of quanta in these terminals can be expected to be very stable. Additionally, the reloading kinetics of SVs with glutamate should be closely linked to the initial intravesicular $\mathrm{Cl}^{-}$concentration just after endocytosis. Since the loading of SVs with glutamate is potentially a limiting step in neurotransmission when rapidly recycling SVs are involved, the utilization of intravesicular $\mathrm{Cl}^{-}$in the translocation process could be a means to ensure fast reloading and therefore stable quantal charge also during prolonged high release rates. However, this assumption has to be confirmed experimentally, e.g. by replacing extracellular $\mathrm{Cl}^{-}$with an impermeable anion under conditions that ensure turnover of SVs in all pools in the necessary dimensions.

\subsection{The lack of enhanced transport by intravesicular $\mathrm{Cl}^{-}$in invertebrate VGLUTs suggests different loading kinetics and smaller quantal size}

The coupling of glutamate entry to the efflux of $\mathrm{Cl}^{-}$in an $\Delta \psi$-driven transport is a bioenergetically coherent explanation for the filling of SVs. Therefore it was tested if this antiport-like transport is evolutionary conserved.

The recently identified DVGLUT was therefore chosen to be tested. The neuromuscular junction where DVGLUT is located is an important model synapse to study the physiology of synaptic transmission (it should be noted that DVGLUT is also expressed in some neurons of the CNS of insects (Daniels et al., 2008)). Further information on the important parameter of vesicle filling would be of interest for investigations regarding synaptic depression or potentiation. For example it has been shown that a single DVGLUT per SV may suffice to charge a SV to full extent (Daniels et al., 2006). It has also been reported that the synapse is keeping the quantal charge constant even if DVGLUT was overexpressed which had impact on the vesicle size (Daniels et al., 2004). The refilling of SVs is thus of great interest not only in vertebrate neurons but also in invertebrate organisms. In addition, very little is known about the specificity and kinetics of DVGLUT since it has never been heterologously expressed. SVs can not be isolated from the 
a

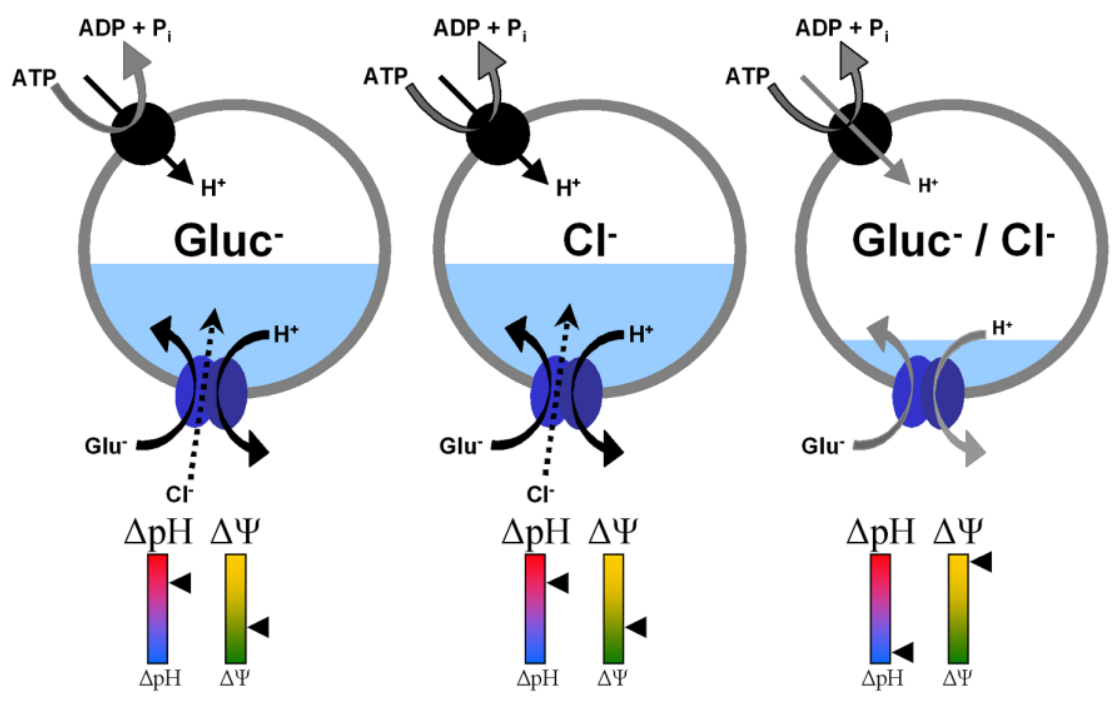

b

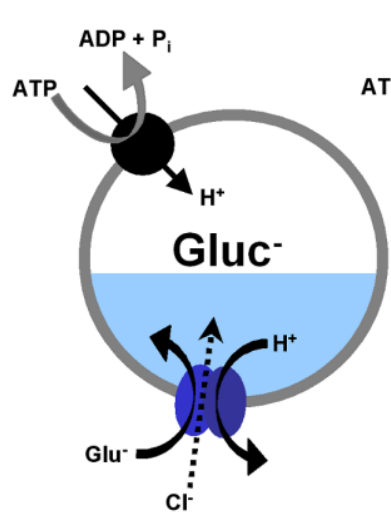

$\Delta \mathrm{pH} \Delta \Psi$

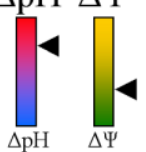

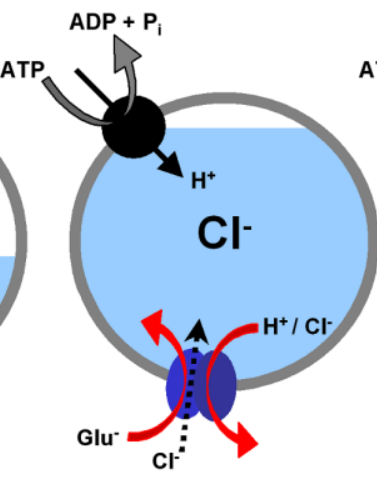

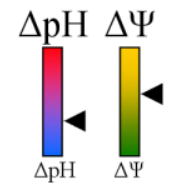

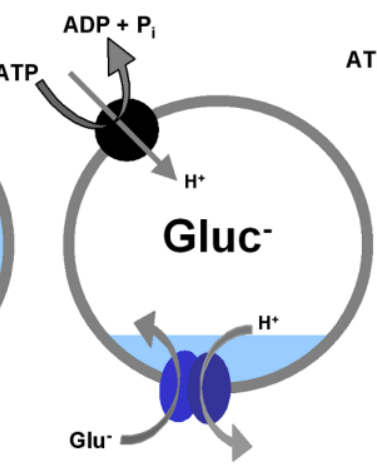

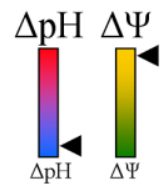

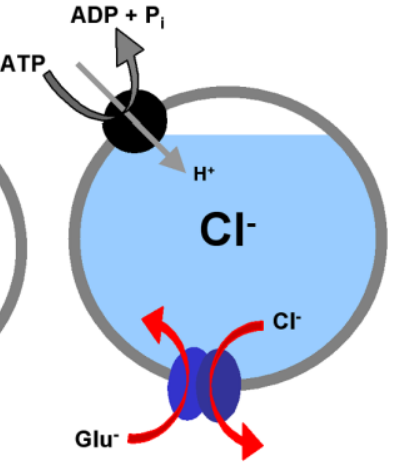

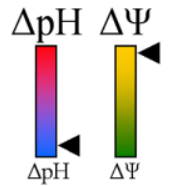

Figure 4.4

Refined model of the loading mechanism by DVGLUT and VGLUT1/2

Schematic representation of the transport modes of DVGLUT (a) and VGLUT1/2 (b) depending on the presence of intravesicular and/or extravesicular $\mathrm{Cl}^{-}$ions. The transport of DVGLUT (a) depends mostly on the presence of extravesicular $\mathrm{Cl}^{-}$and $\Delta \mathrm{pH}$. The internal anion has very little influence on the glutamate translocation. In contrast VGLUT1/2 (b) are enhanced by intravesicular $\mathrm{Cl}^{-}$that activates a $\mathrm{Cl}^{-} / g$ lutamate antiport. The transport in this mode is largely depending on $\Delta \psi$ and almost unaltered by low millimolar concentrations of extravesicular $\mathrm{Cl}^{-}$. In the absence of $\mathrm{Cl}^{-}$in the lumen $\Delta \psi$ is not effective for glutamate translocation. The lack of a $\mathrm{Cl}^{-}$ Iglutamate antiport activity suggests that the $\mathrm{Cl}^{-}$entry is separate from the efflux of $\mathrm{Cl}^{-}$ (illustrated by the black dotted arrow).

VGLUT is represented by the blue ovals and the VATPase by a black circle. High flux is represented by red arrows, intermediate flux by black arrows and weak flux by grey arrows. The strength of $\Delta \mathrm{pH}$ and $\Delta \psi$ in the different settings is illustrated below the sketches by blue-red and green-yellow levels respectively. 
neuromuscular junction and the biochemistry of vesicular glutamate transport in invertebrates is thus inaccessible without heterologous expression.

However, when the transport of purified DVGLUT was tested in the same manner as for VGLUT1, it was apparent that the enhancement by intravesicular was actually very low (Fig. 3.35). The small enhancement by intravesicular $\mathrm{Cl}^{-}$was probably only within the experimental error. Uptake in the absence of extravesicular $\mathrm{Cl}^{-}$was far lower for $\mathrm{Cl}^{-}$loaded vesicles when DVGLUT was compared to VGLUT1 (Fig 3.35 and 3.36a and c). To the authors surprise the antiport model was not valid for DVGLUT. Instead, the characterization of DVGLUT supported a view where DVGLUT operates largely as an $\mathrm{H}^{+}$/glutamate antiporter since it was clearly dependent on extravesicular $\mathrm{Cl}^{-}$and sensitive to nigericin (Fig. 3.36c). However, the transport cycle might still require a $\Delta \psi$ component. Yet, the pronounced sensitivity for nigericin is most compatible with the idea that protons are a substrate during glutamate translocation (this applies also to the $\Delta \mathrm{pH}$ component of VGLUT1).

Another hint for the absence of an $\mathrm{Cl}^{-} /$glutamate antiport in DVGLUT, albeit suffering from a low signal, was the transport of glutamate in $\mathrm{Cl}^{-}$loaded liposomes in the absence of ATP (Figs. 3.23a and 3.34b). DVGLUT had a lower uptake than VGLUT1 under the same conditions when ATP was not added. This low uptake is likely the result of an antiport activity in VGLUT1 and highlights at the same time that the exchange for $\mathrm{Cl}^{-}$and glutamate requires $\Delta \psi$, most likely by powering conformational changes in the transporter protein. Such a background activity was never observed for DVGLUT.

The finding of the absence of a $\mathrm{Cl}^{-} /$glutamate antiport made it interesting to test the uptake in more isoforms. Therefore one more invertebrate VGLUT (eat-4 from C. elegans) was expressed and purified as well as an additional member of the mammalian VGLUTs: VGLUT2 from the mouse. Eat-4 was also not enhanced by intravesicular $\mathrm{Cl}^{-}$, and appeared to be even inhibited by it (Fig. 3.36d). The C. elegans homologue was strictly depending on $\triangle \mathrm{pH}$, thus highly sensitive to nigericin. VGLUT2 showed a very similar uptake pattern as VGLUT1 (Fig. 3.36b) making it likely that the $\mathrm{Cl}^{-} /$glutamate antiport is unique to mammalian species, probably vertebrates in general. It appears that the effect of intravesicular $\mathrm{Cl}^{-}$is even stronger in VGLUT2, however further experiments should confirm this before a clear conclusion can be drawn. The uptake by eat-4 is most outstanding compared to the other isoforms and especially the inhibition by intravesicular 
$\mathrm{Cl}^{-}$is confusing in the physiological context where $\mathrm{Cl}^{-}$is omnipresent. However, the composition of the extracellular fluid is not known to the same detail as in vertebrates. For instance, the interstitial fluid of Ascaris suum contains only about $50 \mathrm{mM} \mathrm{Cl}^{-}$(Thorn and Martin, 1987), clearly different to the $130 \mathrm{mM}$ of the cerebro-spinal fluid of mammals. Without doubt eat-4 would also require deeper investigation to gain more insight into this phenomenon.

It could thus be, although it appears surprising at a first glance, that the invertebrate and vertebrate VGLUTs accomplish glutamate loading in a different manner (see Fig. 4.4). Since the $\mathrm{H}^{+}$/glutamate antiport appears also in the mammalian VGLUTs it is possibly the original transport mechanism and the $\mathrm{Cl}^{\circ} /$ glutamate antiport could have appeared later as a specialization to certain requirements. One noteworthy difference between the invertebrate and vertebrate VGLUTs is their physiological role. In vertebrates, VGLUTs charge vesicles with the major excitatory transmitter of the CNS for interneuronal signalling, while invertebrate VGLUTs excite muscles at the neuromuscular junction. A CNS neuron is surrounded by a highly defined interstitial fluid. The composition is tightly controlled and essential for the proper functioning of neuronal communication. This stable milieu could have favored the usage of $\mathrm{Cl}^{-}$since its concentration is not subject to variations. The extracellular liquid in other tissues must also be relatively stable, however it could be more prone to changes in the ionic composition. The composition of the Drosophila hemolymph for example, could vary just after feeding or under conditions of starvation. Although these changes must be subtle, they probably would interfere with the neurotransmission.

Although it remains currently unknown, one can propose another difference between evertebrate and vertebrate glutamate-type SVs. It is not possible to directly compare DVGLUT and VGLUT1 in their transport rates with reconstituted purified proteins, however, the results imply that DVGLUT would fill a vesicle to lesser extent as VGLUT1. The time course of glutamate uptake for VGLUT1 clearly shows that $\mathrm{Cl}^{-}$filled vesicles contain more glutamate at a given timepoint (Fig 3.23b) than the gluconate filled liposomes. It is currently not possible to prove this assumption but some aspects as described in chapter 4.4 are nevertheless in support of this view. Since the transport by DVGLUT appears to be driven mainly by $\Delta \mathrm{pH}$, most of the pair ions of glutamate would be protons and thus exert an osmotic pressure on the SV membrane that should limit the uptake of glutamate. A model that compares the transport of DVGLUT and mammalian 
VGLUTs is shown schematically in figure 4.4 to summarize the charcteristics of both transporter types.

If these mechanisms indeed appear in vivo and if SVs at the neuromuscular junction of Drosophila and really contain less glutamate than vertebrate SVs, can probably only be answered by the use of transgenic flies that express VGLUT1.

\subsection{Are $\mathrm{Cl}^{-}$shunting and $\mathrm{Cl}^{-} /$glutamate antiport based on different mechanisms in VGLUT1 ?}

DVGLUT vesicles did not show enhanced transport of glutamate by intravesicular $\mathrm{Cl}^{-}$, but still required the presence of extravesicular $\mathrm{Cl}^{-}$to drive uptake. As described in chapter 4.3 and figure 4.2 the stimulating effect of low millimolar concentrations of extravesicular $\mathrm{Cl}^{-}$ can be understood by the entrance of $\mathrm{Cl}^{-}$through VGLUT that leads to the establishment of $\Delta \mathrm{pH}$. The elevated number of protons would then be usable as a substrate in an $\mathrm{H}^{+}$/glutamate exchange. With the results from VGLUT1 it was not clear whether the influx and efflux of $\mathrm{Cl}^{-}$are based on the same mechanism within the transporter. However the results from the DVGLUT-experiments could give a hint to answer this question. Although DVGLUT is not stimulated by $\mathrm{Cl}^{-}$on the luminal side it is enhanced by extravesicular $\mathrm{Cl}^{-}$ in analogous manner to the mammalian VGLUTs. If the model proposed in this work is correct, then this enhancement has to be the result of a $\mathrm{Cl}^{-}$influx. Therefore it was tested if DVGLUT is capable of supporting anion shunting in reconstituted vesicles (Fig 3.37). Indeed $\mathrm{Cl}^{-}$permeates through DVGLUT, which supports the idea that extravesicular $\mathrm{Cl}^{-}$ etsablishes a $\Delta \mathrm{pH}$ component in $\mathrm{SVs}$ that drives uptake. Furthermore this finding is also compatible with the effect of nigericin and does not interfere with the notion that intravesicular $\mathrm{Cl}^{-}$in DVGLUT liposomes has no effect. Instead this result suggests that the entry of $\mathrm{Cl}^{-}$is distinct from the $\mathrm{Cl}^{-}$/glutamate antiport. It could be that $\mathrm{Cl}^{-}$leaks from the extravesicular space through the VGLUTs and that the site for entry is the glutamate binding/entry site. The glutamate binding site is rather specific, since for example aspartate is not transported, however a small anion like $\mathrm{Cl}^{-}$could probably pass. This is further supported by the inhibitory effect of even low millimolar $\mathrm{Cl}^{-}$concentrations upon addition of nigericin in VGLUT1 (Fig 3.35 red dotted lines). Thus it can be concluded that $\mathrm{Cl}^{-}$leaks through the glutamate binding site and, if no nigericin is present, increases the entry of 
protons which drives uptake through the $\mathrm{H}^{+}$/glutamate exchange path. If nigericin is added, the protons would be exchanged immediately for potassium ions and $\mathrm{Cl}^{-}$would compete for glutamate binding without any benefit for the glutamate translocation. It should be noted that this effect is lower if the glutamate concentration is raised, which lowers the chance for $\mathrm{Cl}^{-}$binding (Fig 3.35a-b, red dotted lines), further supporting this idea. The affinity for $\mathrm{Cl}^{-}$must however be much lower than for glutamate because $\mathrm{Cl}^{-}$is added usually at much higher concentrations $\left(40 \mu \mathrm{M}\right.$ glutamate and $\left.4 \mathrm{mM} \mathrm{Cl}^{-}\right)$. Therefore the anion shunting and acidifcation probably results from the entry of very few $\mathrm{Cl}^{-}$ions.

The lack of $\mathrm{Cl}^{-}$/glutamate antiport activity in DVGLUT suggests that a difference in the primary structure renders the mammalian VGLUTs competent for the $\mathrm{Cl}^{-} /$glutamate antiport. The distinct properties of evertebrate and vertebrate VGLUTs therefore offer the possibility to identify residues in the amino acid sequence of VGLUTs that define $\mathrm{H}^{+}$/glutamate and $\mathrm{Cl}^{-} /$glutamate antiport activity respectively. A first approach towards this was undertaken by first narrowing down the TMRs by a topology prediction and subsequently align the sequences of the four investigated VGLUTs (VGLUT1/2, DVGLUT and eat-4) (Fig. 3.38). It was assumed that a difference at or nearby TMRs could be responsible for the altered properties. Probably positively charged residues are necessary for the binding of $\mathrm{Cl}^{-}$that are not present in evertebrate VGLUTs. Although this is a simplified view, it was tested by mutating some candidate lysine or arginine residues in VGLUT1 to the corresponding amino acid in DVGLUT. However no immediate effect could be observed. Only the mutant K401A reduced the uptake in the presence of luminal $\mathrm{Cl}^{-}$compared to the wild type VGLUT1 (Fig. 3.41c). However the pattern was still different from DVGLUT since the sensitivity for nigericin was not very pronounced. The approach also shows that a mutagenesis approach without structural information is rather difficult and also hard to interprete. For instance it is imaginable that a number of residues has to be altered in order to delete the Cl-/glutamate antiport in VGLUT1. 


\section{SUMMARY}

Synaptic vesicles are the central organelle for the quantal release of neurotransmitter in neurons. A crucial aspect of transmitter release is the uptake of neurotransmitters into the luminal space of synaptic vesicles. This is achieved by secondary active transporters that are specific for a certain neurotransmitter. The translocation of all classical neurotransmitters such as acetylcholine, serotonine, $\gamma$-aminobutyric acid and glutamate is driven by the ATP- consuming acticity of a vacuolar-type ATPase (V-ATPase) much like it is the case for the secondary active transport in other membraneous organelles of the cell. The proton translocating activity of the V-ATPase builds up a proton electrochemical gradient $\left(\Delta \mu_{\mathrm{H}+}\right)$ that serves as the energy source for the translocation of solutes. Since the V-ATPase is an electrogenic enzyme complex, ATP hydrolysis is only efficiently coupled to proton translocation in the presence of ion-leaks in the synaptic vesicle membrane to enable counter ion or pair ion movement.

The presence of $\mathrm{a} \mathrm{Cl}^{-}$channel on synaptic vesicles has been experimentally shown (Xie et al., 1989) and is believed to play a critical role in the modulation of the electrochemical gradient $\Delta \mu_{\mathrm{H}+}$ across the bilayer by affecting the relative contribution of the $\mathrm{pH}$ gradient $(\Delta \mathrm{pH})$ and the membrane potential $(\Delta \psi)$ to $\Delta \mu_{\mathrm{H}+}$. This $\mathrm{Cl}^{-}$channel to provides a shunting current that leads to a net accumulation of $\mathrm{HCl}$ in the vesicle lumen (increase of $\Delta \mathrm{pH}$ ) at the expense of $\Delta \psi$. Since the composition of $\Delta \mu_{\mathrm{H}+}$ has been shown to be an important factor for the loading of neurotransmitters (Edwards, 2007), depending on the specific transporter, the molecular identity of the $\mathrm{Cl}^{-}$channel is essential for the understanding of neurotransmitter uptake.

Despite some progress this channel has remained elusive for a long time. With $\mathrm{ClC}-3$, a member of the CLC gene family, an interesting candidate for the $\mathrm{Cl}^{-}$channel on synaptic vesicles was found, since the acidifcation of isolated synaptic vesicles is partly impaired by the loss of ClC-3 (Stobrawa et al., 2001). However, the deletion of the Clcn3 gene in mice leads to severe neurodegeneration including the loss of the hippocampus. These neurodegenerative processes also have an impact on the composition of isolated synaptic vesicles. 
Glutamate, the major excitatory transmitter in the vertebrate brain, is translocated by the recently identified vesicular glutamate transporters (VGLUTs) (Bellocchio et al., 2000; Takamori et al., 2000a) and is believed to be driven primarily by $\Delta \psi$ (Maycox et al., 1988). A prominent feature of the VGLUTs is their biphasic dependence on the extravesicular $\mathrm{Cl}^{-}$ concentration which has been demonstrated for isolated SVs (Naito and Ueda, 1985) as well as for membranes that heterologously express VGLUTs (Bai et al., 2001; Bellocchio et al., 2000; Fremeau et al., 2001). Low millimolar concentrations of 2-4 $\mathrm{mM} \mathrm{Cl}^{-}$enhance the transport of glutamate maximally but high concentrations of extravesicular $\mathrm{Cl}^{-}(10-100$ $\mathrm{mM}$ ) attenuate the uptake. The uptake is also minimal in the absence of extravesicular $\mathrm{Cl}^{-}$. While the reduced uptake in the presence of high concentrations of extravesicular $\mathrm{Cl}^{-}$has been ascribed to the presence of the $\mathrm{Cl}^{-}$channel on synaptic vesicles (which reduces $\Delta \psi$ ), the low uptake in the absence of $\mathrm{Cl}^{-}$remained largely unexplainable although $\Delta \psi$ is maximal under these conditions. Recently, it was shown that VGLUT1 induces a $\mathrm{Cl}^{-}$ conductance in heterologously expressing membranes (Bellocchio et al., 2000). This strong indication of a $\mathrm{Cl}^{-}$conductance in the transporter itself is very interesting in the light of the biphasic dependence of vesicular glutamate transport on extravesicular $\mathrm{Cl}^{-}$. Furthermore, this typical biphasic dependence on $\mathrm{Cl}^{-}$is still present in synaptic vesicles from $\mathrm{Clcn}^{-/}$ mice (Stobrawa et al., 2001), shedding doubts on the proposed function of $\mathrm{ClC}-3$ as a synaptic vesicle $\mathrm{Cl}^{-}$channel.

In order to clarify the contributions of either $\mathrm{ClC}-3$ and VGLUT1 on the $\mathrm{Cl}^{-}$conductance, synaptic vesicles from $\operatorname{Vglut}^{-/}$and $\mathrm{Clcn}^{3^{--}}$mice were compared by measurement of the luminal acidifcation. The results clearly favored a role for VGLUT1 in anion shunting on a large fraction of synaptic vesicles. To verify that the conductance is indeed an intrinsic property of VGLUT1 and to investigate the function of $\mathrm{Cl}^{-}$in the translocation process of glutamate, it was attempted to reconstitute purified VGLUT1 into artificial vesicles. VGLUT1 could be heterologously expressed in tsA201 cells in amounts that enabled a deeper analysis of the transport. Indeed, VGLUT1 exhibited also a $\mathrm{Cl}^{-}$conductance in artficial vesicles.

The influence of the luminal solution of synaptic vesicles on the glutamate uptake can not be studied with native components in biochemical assays. However, synaptic vesicles engulf extracellular fluid upon formation by endocytosis and should thus be filled with 130 $\mathrm{mM} \mathrm{NaCl}$. Therefore, the influence of the intravesicular ion composition of the artificial 
vesicles on the glutamate uptake was investigated, as well as the influence of the extravesicular $\mathrm{Cl}^{-}$concentration. It was found that luminal $\mathrm{Cl}^{-}$greatly enhances the uptake by facilitation of the $\Delta \psi$-driven component. The effect of luminal $\mathrm{Cl}^{-}$was dose dependent suggesting that $\mathrm{Cl}^{-}$is a substrate in the translocation process. This implies that VGLUT1 is a $\mathrm{Cl}^{-} /$glutamate exchanger. The increased speed of vesicle loading ( $\sim 3$-fold) is additionally more compatible with the speed of transmitter reloading at glutamatergic synapses.

Furthermore, the activation and attenuation of the uptake by extravesicular $\mathrm{Cl}^{-}$could be also explained by the newly identified $\mathrm{Cl}^{-}$conductance in VGLUT1 itself. It could be shown that the entry of $\mathrm{Cl}^{-}$through VGLUT1 establishes a $\mathrm{pH}$ gradient that drives the uptake of glutamate, presumably by an $\mathrm{H}^{+}$/ glutamate exchange.

This study revealed previously unknown transport details of the vesicular glutamate translocation by VGLUTs. It provided a biophysically coherent description of transmitter loading and storage, unmasked the $\mathrm{Cl}^{-}$translocator on a major fraction of synaptic vesicles and therefore contributes to our understanding of quantal transmission at the glutamatergic synapse in the vertebrate brain. 


\section{ZUSAMMENFASSUNG}

Nervenzellen setzen Neurotransmitter gequantelt frei (Del Castillo and Katz, 1956). Diese Funktionsweise beruht auf der Speicherung von Neurotransmittern in etwa $40 \mathrm{~nm}$ großen, aus einer Lipiddoppelmembran aufgebauten Vesikeln. Die Membran dieser synaptischen Vesikel fusioniert kontrolliert bei Eintreffen eines Signals mit der Plasmamembran und setzt dabei den Neurotransmitter in den synaptischen Spalt frei. Zahlreiche Proteine sind für die Funktion des synaptischen Vesikels unabdingbar. Zentral sind dabei auch die Proteine welche die Beladung der synaptischen Vesikel durchführen. Sie sind Gegenstand dieser Arbeit. Für ein grundlegendes Verständis der Neurotransmission ist eine solide Kenntnis des Beladungsvorgang essentiell.

Die Aminosäure Glutamat ist, neben ihrer prinzipiellen Funktion als Proteinbaustein, ein weit verbreiteter Neurotransmitter im Tierreich. Bei Wirbeltieren ist sie der mit Abstand häufigste erregende Transmitter im zentralen Nervensystem. Bei Arthropoden ist Glutamat der Transmitter an der neuromuskulären Endplatte. Die speziellen Transportproteine für die Glutamattranslokation stellen die vesikulären Glutamattransporter (VGLUTs) dar (Bellocchio et al., 2000; Takamori et al., 2000a). VGLUTs konzentrieren Glutamat in synaptischen Vesikeln etwa zehnfach gegenüber dem Zytoplasma. VGLUTs sind sekundär aktive Transporter, sie werden durch die Aktivität der vakuolären ATPase (V-ATPase) angetrieben. Die V-ATPase, ein Proteinkomplex, der teils in die Membran integriert ist und strukturell der mitochondrialen ATP-Synthase ähnelt, pumpt, angetrieben durch die Hydrolyse von ATP, Protonen ins Lumen des synaptischen Vesikels und erzeugt damit einen protonenbasierten elektrochemischen Gradienten $\left(\Delta \mu_{\mathrm{H}_{+}}\right)$. Dieser Gradient setzt sich aus einer elektrischen Komponente $(\Delta \Psi)$ und einer chemischen Komponente, dem pHGradienten $(\Delta \mathrm{pH})$, zusammen. Maßgeblich für das Verhältnis dieser Komponenten zueinander ist die Permeabilität der Membran. So ist $\Delta \mu_{\mathrm{H}+}$ in Abwesenheit von Leitfähigkeiten für Gegenionen oder Paarionen ausschließlich aus $\Delta \Psi$ aufgebaut. Befinden sich hingegen Ionenkanäle in der Membran, welche die Passage von Anionen erlauben, säuert sich das Lumen an, da diese Ionen ebenfalls in das Vesikel einströmen um den 
Ladungsausgleich zu gewährleisten. Beide Komponenten von $\Delta \mu_{\mathrm{H}+}$ können als Energiequelle für den Transport von Molekülen an der Vesikelmembran genutzt werden, entscheidend ist dabei das sekundäre Transportprotein (Edwards, 2007). Die Aufnahme von Glutamat wird nach heutiger Sicht hauptsächlich durch $\Delta \Psi$ angetrieben. Dennoch wird ein Austausch mit luminalen Protonen angenommen (Edwards, 2007).

Isolierte synaptische Vesikel lassen sich nach Zugabe von ATP nur dann ansäuern, wenn sich kleine Anionen wie Chlorid in der extravesikulären Lösung befinden. Dies deutet darauf hin, dass sich ein Chlorid-selektiver Ionenkanal in der Membran von synaptischen Vesikeln befindet (Xie et al., 1989). Dieser Chloridkanal blieb lange Zeit unentdeckt. Die oben erwähnte Bedeutung der Zusammensetzung von $\Delta \mu_{\mathrm{H}+}$ für den sekundär aktiven Transport von Neurotransmittern, macht die molekulare Signatur dieses Ionenkanals sehr wichtig, da nur eine genaue Kenntis der Funktionsweise aller Komponenten der Transmitteraufnahme den Vorgang vollständig erklären kann.

Vor einiger Zeit wurde eine Isoform aus der Familie der intrazellulären Chloridkanäle (CLCs) vorgeschlagen, die diese Funktion übernimmt - ClC-3 (Stobrawa et al., 2001). Die Annahme beruht im Wesentlich darauf, dass synaptische Vesikel aus Mäusen bei denen das Gen für ClC-3 ausgeschaltet wurde, nicht mehr effizient angesäuert werden können. Zudem sprach die Lokalisation von ClC-3 für eine solche Rolle, da es in synaptischen Vesikeln angereichert erschien. Problematisch an diesem Befund ist allerdings die Tatsache, dass der Verlust von ClC-3 mit neurodegenerativen Prozessen einhergeht. So bildet sich der gesamte Hippocampus innherhalb weniger Wochen zurück und hinterlässt einen flüssigkeitsgefüllten Hohlraum (Stobrawa et al., 2001). Dies mag als Erklärung dafür dienen, dass sich die Zusammenstzung von isolierten synaptischen Vesikeln aus ClC-3knockout Mäusen nicht mit der von Wildtyp Mäusen deckt. So ist der vesikuläre Glutatmattransporter1 (VGLUT1) unterrepräsentiert, möglicherweise durch einen selektiven Verlust von glutamatergen synaptischen Endigungen.

Der vesikuläre Glutamattransport von isolierten synaptischen Vesikeln zeichnet sich durch eine biphasische Abhängigkeit von extravesikulärem Chlorid aus (Naito and Ueda, 1985). Die Aufnahme ist minimal in Abwesenheit von Chlorid (obgleich $\Delta \Psi$ unter diesen Bedingungen maximal ist), optimal in der Anwesenheit von etwa $4 \mathrm{mM}$ Chlorid und sinkt dann wieder auf minimale Aktivität bei einer extravesikulären Chloridkonzentration von 
100-150 mM ab. Entscheidend ist dabei auch die vorliegende Konzentration von Glutamat, wodurch sich die Verhältnisse etwas zu höheren Chloridkonzentrationen verschieben. Etwa zur gleichen Zeit als die Befunde über ClC-3 publik wurden, erschien eine Arbeit die zeigte, dass VGLUT1 eine Chloridleitfähigkeit in Membranen hervorruft wenn das Protein in sekretorischen Vesikeln einer Zellinie überexprimiert wurde (Bellocchio et al., 2000). Leider konnte diese Leitfähigkeit nur vor dem Hintergrund von anderen, natürlich in der Membran vorkommenden Chloridkanälen gezeigt werden, so dass ein konkreter Schluss damit nicht möglich war. Diese mutmaßliche Leitfähigkeit, zusammen mit der ausgeprägten biphasischen Abhängigkeit von Chlorid lassen es möglich erscheinen, dass VGLUT1 selbst als Chloridtranslokator auf synaptischen Vesikeln operiert. Dies wird auch indirekt dadurch unterstützt, dass der Anteil von VGLUT1 in Präparationen aus ClC-3knockout Mäusen reduziert ist und die biphasische Abhängigkeit von Chlorid bei der Glutamataufnahme ungestört bleibt.

Im Rahmen dieser Arbeit wurden deshalb synaptische Vesikel aus Mäusen bei denen entweder das ClC-3- oder das VGLUT1-Gen deletiert war, hinsichtlich ihrer Chloridleitfähigkeit untersucht. Die Befunde sprachen dabei eindeutig für eine Funktion von VGLUT1 als Chloridtranslokator auf einem großen Teil von synaptischen Vesikeln. Um diese Leitfähigkeit in einem unabhängigen Ansatz zu bestätigen, wurde der Transporter aus Rattus norvegicus heterolog exprimiert, aufgereinigt und dann in künstlichen Membranen zusammen mit einer Protonenpumpe rekonstituiert. Dabei konnte die Leitfähigkeit für Chlorid bestätigt werden. Die Rekonstitution in künstliche Vesikel ermöglichte auch eine tiefergehende Analyse des Glutamattransports, insbesondere die Rolle des Chlorids für die Translokation betreffend. Hierbei wurde festgestellt, dass die biphasische Anhängigkeit des Transports auf den Eintritt von Chloridionen in das Vesikellumen durch VGLUT1 zurückzuführen ist. Dabei wird ein pH-Gradient etabliert, der wahrscheinlich einem Proton-Glutamat-Antiport in VGLUT1 als Energiequelle dient. Ein Befund mit weitreichenden Folgen war außerdem, dass der Transport von Glutamat stark beschleunigt ablief und auch eine höhere Aufnahme an Glutamat ermöglichte, wenn im Lumen des Vesikels Chlorid vorlag. Diese Versuchsbedingung war ohne aufgereinigte Proteine und künstliche Vesikeln nicht realisierbar. Das Phänomen hängt dabei von der Konzentration des Chlorids im Vesikellumen ab, daher ist es wahrscheinlich das VGLUT1 auch als Chlorid-Glutamat-Antiporter fungieren kann, eine bislang nur vermutete 
Funktionsweise (Maycox et al., 1990b). Da synaptische Vesikel bei ihrer Regeneration an der Plasmamembran extrazelluläre Flüssigkeit aufnehmen, wird vermutlich die Kapazität der Glutamataufnahme in synaptischen Vesikeln, insbesondere aber auch die Geschwindigkeit, maßgeblich durch die extrazelluläre Chloridkonzentration beeinflusst, wenn nicht gar determiniert.

Diese Entdeckung trägt somit $\mathrm{zu}$ unserem Kenntisstand der gequantelten Neurotransmission an der glutamatergen Synapse bei und hat weitreichende Konsequenzen für das Verständnis der Glutamataufnahme bei hohem Vesikelumsatz sowie der Größe einzelner Transmitterquanten, zweier entscheidender Parameter für die Physiologie der Synapse. 


\section{7. 要約}

シナプス小胞へのグルタミン酸の取り込みは Vesicular Glutamate Transporter (VGLUT)によってなされる。この過程はクロライドイオンに依存していることが わかっているが、このイオンがどのように取り込みに関与しているかは明らかに はなっていない。以前より、CIC3 がシナプス小胞でのクロライドイオンの透過性 を制御していると信じられてきたが、本研究で VGLUT1 がそれを規定しているこ とが明らかにされた。私たちはリコンビナントVGLUT1 を用いた生化学的再構成 系により VGLUT1 自身がシナプス小胞内外のクロライドイオンの透過させること、 シナプス小胞外クロライド濃度により二相性にグルタミン酸の取り込みが制御さ れることを明らかにした。さらに、小胞内クロライド濃度が高めると膜電位依存 的にグルタミン酸の取り込みを顕著に促進することを発見した。これは従来認識 されたいた VGLUT1の取り込み機構とは異なるものである。エンドサイトーシス 後のシナプス小胞は高濃度のクロライドイオンを含んでいることから、グルタミ ン酸の取り込み速度と最終的小胞内濃度は細胞外クロライド濃度に依存している ことが示唆された。 


\section{REFERENCES}

Abramson, J., Smirnova, I., Kasho, V., Verner, G., Kaback, H.R., and Iwata, S. (2003). Structure and mechanism of the lactose permease of Escherichia coli. Science 301, 610-615.

Accardi, A., Kolmakova-Partensky, L., Williams, C., and Miller, C. (2004). Ionic currents mediated by a prokaryotic homologue of CLC Cl- channels. J Gen Physiol 123, 109-119.

Accardi, A., and Miller, C. (2004). Secondary active transport mediated by a prokaryotic homologue of ClC Cl- channels. Nature 427, 803-807.

Aihara, Y., Mashima, H., Onda, H., Hisano, S., Kasuya, H., Hori, T., Yamada, S., Tomura, H., Yamada, Y., Inoue, I., et al. (2000). Molecular cloning of a novel brain-type $\mathrm{Na}(+)$-dependent inorganic phosphate cotransporter. J Neurochem 74, 2622-2625.

Almqvist, J., Huang, Y., Laaksonen, A., Wang, D.N., and Hovmoller, S. (2007). Docking and homology modeling explain inhibition of the human vesicular glutamate transporters. Protein Sci $16,1819-1829$.

Avery, L. (1993). The genetics of feeding in Caenorhabditis elegans. Genetics 133, 897-917.

Axelsson, M.A., Karlsson, N.G., Steel, D.M., Ouwendijk, J., Nilsson, T., and Hansson, G.C. (2001). Neutralization of $\mathrm{pH}$ in the Golgi apparatus causes redistribution of glycosyltransferases and changes in the O-glycosylation of mucins. Glycobiology 11, 633-644.

Bai, L., Xu, H., Collins, J.F., and Ghishan, F.K. (2001). Molecular and functional analysis of a novel neuronal vesicular glutamate transporter. J Biol Chem 276, 36764-36769.

Baumert, M., Maycox, P.R., Navone, F., De Camilli, P., and Jahn, R. (1989). Synaptobrevin: an integral membrane protein of 18,000 daltons present in small synaptic vesicles of rat brain. EMBO J 8, 379-384.

Bayer, M.J., Reese, C., Buhler, S., Peters, C., and Mayer, A. (2003). Vacuole membrane fusion: V0 functions after trans-SNARE pairing and is coupled to the Ca2+-releasing channel. J Cell Biol 162, 211-222. 


\section{REFERENCES}

Bellocchio, E.E., Hu, H., Pohorille, A., Chan, J., Pickel, V.M., and Edwards, R.H. (1998). The localization of the brain-specific inorganic phosphate transporter suggests a specific presynaptic role in glutamatergic transmission. J Neurosci 18, 8648-8659.

Bellocchio, E.E., Reimer, R.J., Fremeau, R.T., Jr., and Edwards, R.H. (2000). Uptake of glutamate into synaptic vesicles by an inorganic phosphate transporter. Science 289, 957-960.

Boulland, J.L., Qureshi, T., Seal, R.P., Rafiki, A., Gundersen, V., Bergersen, L.H., Fremeau, R.T., Jr., Edwards, R.H., Storm-Mathisen, J., and Chaudhry, F.A. (2004). Expression of the vesicular glutamate transporters during development indicates the widespread corelease of multiple neurotransmitters. J Comp Neurol 480, 264-280.

Brose, N. (2008). For better or for worse: complexins regulate SNARE function and vesicle fusion. Traffic 9, 1403-1413.

Brose, N., Thomas, A., Weber, M.G., and Jahn, R. (1990). A chloride- and calcium-dependent glutamate-binding protein from rat brain. Identification as a ubiquitous constituent of the inner mitochondrial membrane. J Biol Chem 265, 10604-10610.

Brunk, I., Holtje, M., von Jagow, B., Winter, S., Sternberg, J., Blex, C., Pahner, I., and AhnertHilger, G. (2006). Regulation of vesicular monoamine and glutamate transporters by vesicleassociated trimeric $\mathrm{G}$ proteins: new jobs for long-known signal transduction molecules. Handb Exp Pharmacol, 305-325.

Bruns, D., and Jahn, R. (1995). Real-time measurement of transmitter release from single synaptic vesicles. Nature 377, 62-65.

Bruns, D., Riedel, D., Klingauf, J., and Jahn, R. (2000). Quantal release of serotonin. Neuron 28, 205-220.

Burger, P.M., Hell, J., Mehl, E., Krasel, C., Lottspeich, F., and Jahn, R. (1991). GABA and glycine in synaptic vesicles: storage and transport characteristics. Neuron 7, 287-293. 


\section{REFERENCES}

Burger, P.M., Mehl, E., Cameron, P.L., Maycox, P.R., Baumert, M., Lottspeich, F., De Camilli, P., and Jahn, R. (1989). Synaptic vesicles immunoisolated from rat cerebral cortex contain high levels of glutamate. Neuron 3, 715-720.

Chen, C., and Okayama, H. (1987). High-efficiency transformation of mammalian cells by plasmid DNA. Mol Cell Biol 7, 2745-2752.

Cidon, S., Ben-David, H., and Nelson, N. (1983). ATP-driven proton fluxes across membranes of secretory organelles. J Biol Chem 258, 11684-11688.

Danbolt, N.C. (2001). Glutamate uptake. Prog Neurobiol 65, 1-105.

Daniels, R.W., Collins, C.A., Chen, K., Gelfand, M.V., Featherstone, D.E., and DiAntonio, A. (2006). A single vesicular glutamate transporter is sufficient to fill a synaptic vesicle. Neuron 49 , $11-16$

Daniels, R.W., Collins, C.A., Gelfand, M.V., Dant, J., Brooks, E.S., Krantz, D.E., and DiAntonio, A. (2004). Increased expression of the Drosophila vesicular glutamate transporter leads to excess glutamate release and a compensatory decrease in quantal content. J Neurosci 24, 10466-10474.

Daniels, R.W., Gelfand, M.V., Collins, C.A., and DiAntonio, A. (2008). Visualizing glutamatergic cell bodies and synapses in Drosophila larval and adult CNS. J Comp Neurol 508, 131-152.

De Gois, S., Jeanclos, E., Morris, M., Grewal, S., Varoqui, H., and Erickson, J.D. (2006). Identification of endophilins 1 and 3 as selective binding partners for VGLUT1 and their colocalization in neocortical glutamatergic synapses: implications for vesicular glutamate transporter trafficking and excitatory vesicle formation. Cell Mol Neurobiol 26, 679-693.

Degrip, W.J., Vanoostrum, J., and Bovee-Geurts, P.H. (1998). Selective detergent-extraction from mixed detergent/lipid/protein micelles, using cyclodextrin inclusion compounds: a novel generic approach for the preparation of proteoliposomes. Biochem J 330 ( Pt 2), 667-674.

Del Castillo, J., and Katz, B. (1956). Biophysical aspects of neuro-muscular transmission. Prog Biophys Biophys Chem 6, 121-170. 


\section{REFERENCES}

Dent, J.A., Davis, M.W., and Avery, L. (1997). avr-15 encodes a chloride channel subunit that mediates inhibitory glutamatergic neurotransmission and ivermectin sensitivity in Caenorhabditis elegans. EMBO J 16, 5867-5879.

Di Paolo, G., and De Camilli, P. (2006). Phosphoinositides in cell regulation and membrane dynamics. Nature 443, 651-657.

Dickerson, L.W., Bonthius, D.J., Schutte, B.C., Yang, B., Barna, T.J., Bailey, M.C., Nehrke, K., Williamson, R.A., and Lamb, F.S. (2002). Altered GABAergic function accompanies hippocampal degeneration in mice lacking ClC-3 voltage-gated chloride channels. Brain Res 958, 227-250.

Disbrow, J.K., Gershten, M.J., and Ruth, J.A. (1982). Uptake of L-[3H] glutamic acid by crude and purified synaptic vesicles from rat brain. Biochem Biophys Res Commun 108, 1221-1227.

Doherty, G.J., and McMahon, H.T. (2009). Mechanisms of Endocytosis. Annu Rev Biochem.

Dong, M., Yeh, F., Tepp, W.H., Dean, C., Johnson, E.A., Janz, R., and Chapman, E.R. (2006). SV2 is the protein receptor for botulinum neurotoxin A. Science 312, 592-596.

Dutzler, R., Campbell, E.B., Cadene, M., Chait, B.T., and MacKinnon, R. (2002). X-ray structure of a $\mathrm{ClC}$ chloride channel at 3.0 A reveals the molecular basis of anion selectivity. Nature 415, 287-294.

Edwards, R.H. (2007). The neurotransmitter cycle and quantal size. Neuron 55, 835-858.

Feany, M.B., Lee, S., Edwards, R.H., and Buckley, K.M. (1992). The synaptic vesicle protein SV2 is a novel type of transmembrane transporter. Cell 70, 861-867.

Fei, H., Karnezis, T., Reimer, R.J., and Krantz, D.E. (2007). Membrane topology of the Drosophila vesicular glutamate transporter. J Neurochem 101, 1662-1671.

Fernandez-Chacon, R., Konigstorfer, A., Gerber, S.H., Garcia, J., Matos, M.F., Stevens, C.F., Brose, N., Rizo, J., Rosenmund, C., and Sudhof, T.C. (2001). Synaptotagmin I functions as a calcium regulator of release probability. Nature 410, 41-49. 


\section{REFERENCES}

Fon, E.A., Pothos, E.N., Sun, B.C., Killeen, N., Sulzer, D., and Edwards, R.H. (1997). Vesicular transport regulates monoamine storage and release but is not essential for amphetamine action. Neuron 19, 1271-1283.

Fremeau, R.T., Jr., Burman, J., Qureshi, T., Tran, C.H., Proctor, J., Johnson, J., Zhang, H., Sulzer, D., Copenhagen, D.R., Storm-Mathisen, J., et al. (2002). The identification of vesicular glutamate transporter 3 suggests novel modes of signaling by glutamate. Proc Natl Acad Sci U S A 99, 14488-14493.

Fremeau, R.T., Jr., Kam, K., Qureshi, T., Johnson, J., Copenhagen, D.R., Storm-Mathisen, J., Chaudhry, F.A., Nicoll, R.A., and Edwards, R.H. (2004). Vesicular glutamate transporters 1 and 2 target to functionally distinct synaptic release sites. Science 304, 1815-1819.

Fremeau, R.T., Jr., Troyer, M.D., Pahner, I., Nygaard, G.O., Tran, C.H., Reimer, R.J., Bellocchio, E.E., Fortin, D., Storm-Mathisen, J., and Edwards, R.H. (2001). The expression of vesicular glutamate transporters defines two classes of excitatory synapse. Neuron 31, 247-260.

Friedrich, T., Breiderhoff, T., and Jentsch, T.J. (1999). Mutational analysis demonstrates that ClC-4 and ClC-5 directly mediate plasma membrane currents. J Biol Chem 274, 896-902.

Fujiyama, F., Furuta, T., and Kaneko, T. (2001). Immunocytochemical localization of candidates for vesicular glutamate transporters in the rat cerebral cortex. J Comp Neurol 435, 379-387.

Garcia-Garcia, A.L., Elizalde, N., Matrov, D., Harro, J., Wojcik, S.M., Venzala, E., Ramirez, M.J., Rio, J.D., and Tordera, R.M. (2009). Increased Vulnerability to Depressive-Like Behavior of Mice with Decreased Expression of VGLUT1. Biol Psychiatry.

Gerschenfeld, H.M. (1973). Chemical transmission in invertebrate central nervous systems and neuromuscular junctions. Physiol Rev 53, 1-119.

Giompres, P.E., Zimmermann, H., and Whittaker, V.P. (1981). Purification of small dense vesicles from stimulated Torpedo electric tissue by glass bead column chromatography. Neuroscience 6 , 765-774. 


\section{REFERENCES}

Glinn, M., Ni, B., Irwin, R.P., Kelley, S.W., Lin, S.Z., and Paul, S.M. (1998). Inorganic Pi increases neuronal survival in the acute early phase following excitotoxic/oxidative insults. $\mathbf{J}$ Neurochem 70, 1850-1858.

Grabe, M., and Oster, G. (2001). Regulation of organelle acidity. J Gen Physiol 117, 329-344.

Gras, C., Amilhon, B., Lepicard, E.M., Poirel, O., Vinatier, J., Herbin, M., Dumas, S., Tzavara, E.T., Wade, M.R., Nomikos, G.G., et al. (2008). The vesicular glutamate transporter VGLUT3 synergizes striatal acetylcholine tone. Nat Neurosci 11, 292-300.

Gras, C., Herzog, E., Bellenchi, G.C., Bernard, V., Ravassard, P., Pohl, M., Gasnier, B., Giros, B., and El Mestikawy, S. (2002). A third vesicular glutamate transporter expressed by cholinergic and serotoninergic neurons. J Neurosci 22, 5442-5451.

Graves, A.R., Curran, P.K., Smith, C.L., and Mindell, J.A. (2008). The Cl-/H+ antiporter ClC-7 is the primary chloride permeation pathway in lysosomes. Nature 453, 788-792.

Greengard, P., Benfenati, F., and Valtorta, F. (1994). Synapsin I, an actin-binding protein regulating synaptic vesicle traffic in the nerve terminal. Adv Second Messenger Phosphoprotein Res 29, 31-45.

Hara-Chikuma, M., Wang, Y., Guggino, S.E., Guggino, W.B., and Verkman, A.S. (2005a). Impaired acidification in early endosomes of $\mathrm{ClC}-5$ deficient proximal tubule. Biochem Biophys Res Commun 329, 941-946.

Hara-Chikuma, M., Yang, B., Sonawane, N.D., Sasaki, S., Uchida, S., and Verkman, A.S. (2005b). $\mathrm{ClC}-3$ chloride channels facilitate endosomal acidification and chloride accumulation. J Biol Chem $280,1241-1247$.

Hartinger, J., and Jahn, R. (1993). An anion binding site that regulates the glutamate transporter of synaptic vesicles. J Biol Chem 268, 23122-23127.

Hell, J.W., Edelmann, L., Hartinger, J., and Jahn, R. (1991). Functional reconstitution of the gamma-aminobutyric acid transporter from synaptic vesicles using artificial ion gradients. Biochemistry 30, 11795-11800. 


\section{REFERENCES}

Hell, J.W., and Jahn, R. in Cell Biology: A Laboratory Handbook $1^{\text {st }}$ edn (ed. Celis, J.E.) 567-574 (Academic Press, New York, 1994).

Hell, J.W., and Jahn, R. (1998). Bioenergetic characterization of gamma-aminobutyric acid transporter of synaptic vesicles. Methods Enzymol 296, 116-124.

Hell, J.W., Maycox, P.R., and Jahn, R. (1990). Energy dependence and functional reconstitution of the gamma-aminobutyric acid carrier from synaptic vesicles. J Biol Chem 265, 2111-2117.

Herzog, E., Bellenchi, G.C., Gras, C., Bernard, V., Ravassard, P., Bedet, C., Gasnier, B., Giros, B., and El Mestikawy, S. (2001). The existence of a second vesicular glutamate transporter specifies subpopulations of glutamatergic neurons. J Neurosci 21, RC181.

Herzog, E., Takamori, S., Jahn, R., Brose, N., and Wojcik, S.M. (2006). Synaptic and vesicular colocalization of the glutamate transporters VGLUT1 and VGLUT2 in the mouse hippocampus. J Neurochem 99, 1011-1018.

Heuser, J.E., and Reese, T.S. (1973). Evidence for recycling of synaptic vesicle membrane during transmitter release at the frog neuromuscular junction. J Cell Biol 57, 315-344.

Heuser, J.E., Reese, T.S., Dennis, M.J., Jan, Y., Jan, L., and Evans, L. (1979). Synaptic vesicle exocytosis captured by quick freezing and correlated with quantal transmitter release. J Cell Biol $81,275-300$.

Hirata, T., Nakamura, N., Omote, H., Wada, Y., and Futai, M. (2000). Regulation and reversibility of vacuolar H(+)-ATPase. J Biol Chem 275, 386-389.

Holroyd, C., Kistner, U., Annaert, W., and Jahn, R. (1999). Fusion of endosomes involved in synaptic vesicle recycling. Mol Biol Cell 10, 3035-3044.

Huttner, W.B., Schiebler, W., Greengard, P., and De Camilli, P. (1983). Synapsin I (protein I), a nerve terminal-specific phosphoprotein. III. Its association with synaptic vesicles studied in a highly purified synaptic vesicle preparation. J Cell Biol 96, 1374-1388. 


\section{REFERENCES}

Ikeda, K., and Bekkers, J.M. (2009). Counting the number of releasable synaptic vesicles in a presynaptic terminal. Proc Natl Acad Sci U S A 106, 2945-2950.

Inoue, T., Wang, Y., Jefferies, K., Qi, J., Hinton, A., and Forgac, M. (2005). Structure and regulation of the V-ATPases. J Bioenerg Biomembr 37, 393-398.

Iyer, R., Iverson, T.M., Accardi, A., and Miller, C. (2002). A biological role for prokaryotic ClC chloride channels. Nature 419, 715-718.

Jahn, R. (2000). Sec1/Munc18 proteins: mediators of membrane fusion moving to center stage. Neuron 27, 201-204.

Jahn, R., and Scheller, R.H. (2006). SNAREs--engines for membrane fusion. Nat Rev Mol Cell Biol 7, 631-643.

Jahr, C.E., and Lester, R.A. (1992). Synaptic excitation mediated by glutamate-gated ion channels. Curr Opin Neurobiol 2, 270-274.

Janz, R., Goda, Y., Geppert, M., Missler, M., and Sudhof, T.C. (1999a). SV2A and SV2B function as redundant $\mathrm{Ca} 2+$ regulators in neurotransmitter release. Neuron 24, 1003-1016.

Janz, R., Sudhof, T.C., Hammer, R.E., Unni, V., Siegelbaum, S.A., and Bolshakov, V.Y. (1999b). Essential roles in synaptic plasticity for synaptogyrin I and synaptophysin I. Neuron 24, 687-700.

Jentsch, T.J. (1996). Chloride channels: a molecular perspective. Curr Opin Neurobiol 6, 303-310.

Jentsch, T.J., Poet, M., Fuhrmann, J.C., and Zdebik, A.A. (2005). Physiological functions of CLC Cl- channels gleaned from human genetic disease and mouse models. Annu Rev Physiol 67, 779807.

Jentsch, T.J., Steinmeyer, K., and Schwarz, G. (1990). Primary structure of Torpedo marmorata chloride channel isolated by expression cloning in Xenopus oocytes. Nature 348, 510-514.

Johnson, R.G., Carty, S.E., and Scarpa, A. (1981). Proton: substrate stoichiometries during active transport of biogenic amines in chromaffin ghosts. J Biol Chem 256, 5773-5780. 


\section{REFERENCES}

Juge, N., Yoshida, Y., Yatsushiro, S., Omote, H., and Moriyama, Y. (2006). Vesicular glutamate transporter contains two independent transport machineries. J Biol Chem 281, 39499-39506.

Kasper, D., Planells-Cases, R., Fuhrmann, J.C., Scheel, O., Zeitz, O., Ruether, K., Schmitt, A., Poet, M., Steinfeld, R., Schweizer, M., et al. (2005). Loss of the chloride channel ClC-7 leads to lysosomal storage disease and neurodegeneration. EMBO J 24, 1079-1091.

Kawasaki, M., Uchida, S., Monkawa, T., Miyawaki, A., Mikoshiba, K., Marumo, F., and Sasaki, S. (1994). Cloning and expression of a protein kinase C-regulated chloride channel abundantly expressed in rat brain neuronal cells. Neuron 12, 597-604.

Keefe, A.D., Wilson, D.S., Seelig, B., and Szostak, J.W. (2001). One-step purification of recombinant proteins using a nanomolar-affinity streptavidin-binding peptide, the SBP-Tag. Protein Expr Purif 23, 440-446.

Knoth, J., Zallakian, M., and Njus, D. (1981). Stoichiometry of H+-linked dopamine transport in chromaffin granule ghosts. Biochemistry 20, 6625-6629.

Kornak, U., Kasper, D., Bosl, M.R., Kaiser, E., Schweizer, M., Schulz, A., Friedrich, W., Delling, G., and Jentsch, T.J. (2001). Loss of the ClC-7 chloride channel leads to osteopetrosis in mice and man. Cell 104, 205-215.

Laemmli, U.K. (1970). Cleavage of structural proteins during the assembly of the head of bacteriophage T4. Nature 227, 680-685.

Lewis, S.M., and Ueda, T. (1998). Solubilization and reconstitution of synaptic vesicle glutamate transport system. Methods Enzymol 296, 125-144.

Lynch, B.A., Lambeng, N., Nocka, K., Kensel-Hammes, P., Bajjalieh, S.M., Matagne, A., and Fuks, B. (2004). The synaptic vesicle protein SV2A is the binding site for the antiepileptic drug levetiracetam. Proc Natl Acad Sci U S A 101, 9861-9866.

Maeda, Y., Ide, T., Koike, M., Uchiyama, Y., and Kinoshita, T. (2008). GPHR is a novel anion channel critical for acidification and functions of the Golgi apparatus. Nat Cell Biol 10, 1135-1145. 


\section{REFERENCES}

Martens, H., Weston, M.C., Boulland, J.L., Gronborg, M., Grosche, J., Kacza, J., Hoffmann, A., Matteoli, M., Takamori, S., Harkany, T., et al. (2008). Unique luminal localization of VGAT-C terminus allows for selective labeling of active cortical GABAergic synapses. J Neurosci 28, 13125-13131.

Martens, S., and McMahon, H.T. (2008). Mechanisms of membrane fusion: disparate players and common principles. Nat Rev Mol Cell Biol 9, 543-556.

Matsuda, J.J., Filali, M.S., Volk, K.A., Collins, M.M., Moreland, J.G., and Lamb, F.S. (2008). Overexpression of CLC-3 in HEK293T cells yields novel currents that are $\mathrm{pH}$ dependent. Am J Physiol Cell Physiol 294, C251-262.

Maxfield, F.R., and McGraw, T.E. (2004). Endocytic recycling. Nat Rev Mol Cell Biol 5, 121-132.

Maycox, P.R., Deckwerth, T., Hell, J.W., and Jahn, R. (1988). Glutamate uptake by brain synaptic vesicles. Energy dependence of transport and functional reconstitution in proteoliposomes. J Biol Chem 263, 15423-15428.

Maycox, P.R., Deckwerth, T., and Jahn, R. (1990a). Bacteriorhodopsin drives the glutamate transporter of synaptic vesicles after co-reconstitution. EMBO J 9, 1465-1469.

Maycox, P.R., Hell, J.W., and Jahn, R. (1990b). Amino acid neurotransmission: spotlight on synaptic vesicles. Trends Neurosci 13, 83-87.

McIntire, S.L., Jorgensen, E., and Horvitz, H.R. (1993). Genes required for GABA function in Caenorhabditis elegans. Nature 364, 334-337.

McIntire, S.L., Reimer, R.J., Schuske, K., Edwards, R.H., and Jorgensen, E.M. (1997). Identification and characterization of the vesicular GABA transporter. Nature 389, 870-876.

Meyer, S., Savaresi, S., Forster, I.C., and Dutzler, R. (2007). Nucleotide recognition by the cytoplasmic domain of the human chloride transporter ClC-5. Nat Struct Mol Biol 14, 60-67. 


\section{REFERENCES}

Miesenbock, G., De Angelis, D.A., and Rothman, J.E. (1998). Visualizing secretion and synaptic transmission with $\mathrm{pH}$-sensitive green fluorescent proteins. Nature 394, 192-195.

Miller, C. (1982). Open-state substructure of single chloride channels from Torpedo electroplax. Philos Trans R Soc Lond B Biol Sci 299, 401-411.

Miyaji, T., Echigo, N., Hiasa, M., Senoh, S., Omote, H., and Moriyama, Y. (2008). Identification of a vesicular aspartate transporter. Proc Natl Acad Sci U S A 105, 11720-11724.

Moechars, D., Weston, M.C., Leo, S., Callaerts-Vegh, Z., Goris, I., Daneels, G., Buist, A., Cik, M., van der Spek, P., Kass, S., et al. (2006). Vesicular glutamate transporter VGLUT2 expression levels control quantal size and neuropathic pain. J Neurosci 26, 12055-12066.

Morin, P., Sagne, C., and Gasnier, B. (2004). Functional characterization of wild-type and mutant human sialin. EMBO J 23, 4560-4570.

Moriyama, Y., Iwamoto, A., Hanada, H., Maeda, M., and Futai, M. (1991). One-step purification of Escherichia coli $\mathrm{H}(+)$-ATPase (F0F1) and its reconstitution into liposomes with neurotransmitter transporters. J Biol Chem 266, 22141-22146.

Moriyama, Y., and Nelson, N. (1987). The purified ATPase from chromaffin granule membranes is an anion-dependent proton pump. J Biol Chem 262, 9175-9180.

Moriyama, Y., and Yamamoto, A. (1995). Vesicular L-glutamate transporter in microvesicles from bovine pineal glands. Driving force, mechanism of chloride anion activation, and substrate specificity. J Biol Chem 270, 22314-22320.

Muller, M., Irkens-Kiesecker, U., Rubinstein, B., and Taiz, L. (1996). On the mechanism of hyperacidification in lemon. Comparison of the vacuolar $\mathrm{H}(+)$-ATPase activities of fruits and epicotyls. J Biol Chem 271, 1916-1924.

Nagy, A., Baker, R.R., Morris, S.J., and Whittaker, V.P. (1976). The preparation and characterization of synaptic vesicles of high purity. Brain Res 109, 285-309. 


\section{REFERENCES}

Naito, S., and Ueda, T. (1985). Characterization of glutamate uptake into synaptic vesicles. J Neurochem 44, 99-109.

Nakamura, K., Hioki, H., Fujiyama, F., and Kaneko, T. (2005). Postnatal changes of vesicular glutamate transporter (VGluT)1 and VGluT2 immunoreactivities and their colocalization in the mouse forebrain. J Comp Neurol 492, 263-288.

Nguyen, M.L., Cox, G.D., and Parsons, S.M. (1998). Kinetic parameters for the vesicular acetylcholine transporter: two protons are exchanged for one acetylcholine. Biochemistry 37, 13400-13410.

Ni, B., Rosteck, P.R., Jr., Nadi, N.S., and Paul, S.M. (1994). Cloning and expression of a cDNA encoding a brain-specific $\mathrm{Na}(+)$-dependent inorganic phosphate cotransporter. Proc Natl Acad Sci U S A 91, 5607-5611.

Ni, B., Wu, X., Yan, G.M., Wang, J., and Paul, S.M. (1995). Regional expression and cellular localization of the $\mathrm{Na}(+)$-dependent inorganic phosphate cotransporter of rat brain. J Neurosci 15 , 5789-5799.

Pankratov, Y., Lalo, U., Verkhratsky, A., and North, R.A. (2006). Vesicular release of ATP at central synapses. Pflugers Arch 452, 589-597.

Parsons, S.M., Prior, C., and Marshall, I.G. (1993). Acetylcholine transport, storage, and release. Int Rev Neurobiol 35, 279-390.

Picollo, A., and Pusch, M. (2005). Chloride/proton antiporter activity of mammalian CLC proteins ClC-4 and ClC-5. Nature 436, 420-423.

Piwon, N., Gunther, W., Schwake, M., Bosl, M.R., and Jentsch, T.J. (2000). ClC-5 Cl- -channel disruption impairs endocytosis in a mouse model for Dent's disease. Nature 408, 369-373.

Pobbati, A.V., Stein, A., and Fasshauer, D. (2006). N- to C-terminal SNARE complex assembly promotes rapid membrane fusion. Science 313, 673-676. 


\section{REFERENCES}

Pothos, E.N., Larsen, K.E., Krantz, D.E., Liu, Y., Haycock, J.W., Setlik, W., Gershon, M.D., Edwards, R.H., and Sulzer, D. (2000). Synaptic vesicle transporter expression regulates vesicle phenotype and quantal size. J Neurosci 20, 7297-7306.

Presley, J.F., Mayor, S., McGraw, T.E., Dunn, K.W., and Maxfield, F.R. (1997). Bafilomycin A1 treatment retards transferrin receptor recycling more than bulk membrane recycling. J Biol Chem 272, 13929-13936.

Price, G.D., and Trussell, L.O. (2006). Estimate of the chloride concentration in a central glutamatergic terminal: a gramicidin perforated-patch study on the calyx of Held. J Neurosci 26, $11432-11436$.

Puri, S., Bachert, C., Fimmel, C.J., and Linstedt, A.D. (2002). Cycling of early Golgi proteins via the cell surface and endosomes upon lumenal pH disruption. Traffic 3, 641-653.

Rizo, J., and Rosenmund, C. (2008). Synaptic vesicle fusion. Nat Struct Mol Biol 15, 665-674.

Rizzoli, S.O., Bethani, I., Zwilling, D., Wenzel, D., Siddiqui, T.J., Brandhorst, D., and Jahn, R. (2006). Evidence for early endosome-like fusion of recently endocytosed synaptic vesicles. Traffic 7, 1163-1176.

Roseth, S., Fykse, E.M., and Fonnum, F. (1995). Uptake of L-glutamate into rat brain synaptic vesicles: effect of inhibitors that bind specifically to the glutamate transporter. J Neurochem 65, 96103.

Ruivo, R., Anne, C., Sagne, C., and Gasnier, B. (2009). Molecular and cellular basis of lysosomal transmembrane protein dysfunction. Biochim Biophys Acta 1793, 636-649.

Ryan, R.M., and Mindell, J.A. (2007). The uncoupled chloride conductance of a bacterial glutamate transporter homolog. Nat Struct Mol Biol 14, 365-371.

Sambrook, J. and Russell, D.W.: Molecular Cloning: A Laboratory Manual. $3^{\text {rd }}$ ed, Cold Spring Harbor, N.Y.: Cold Spring Harbor Laboratory Press, 2001 


\section{REFERENCES}

Sawada, K., Echigo, N., Juge, N., Miyaji, T., Otsuka, M., Omote, H., Yamamoto, A., and Moriyama, Y. (2008). Identification of a vesicular nucleotide transporter. Proc Natl Acad Sci U S A $105,5683-5686$.

Schafer, M.K., Varoqui, H., Defamie, N., Weihe, E., and Erickson, J.D. (2002). Molecular cloning and functional identification of mouse vesicular glutamate transporter 3 and its expression in subsets of novel excitatory neurons. J Biol Chem 277, 50734-50748.

Scheel, O., Zdebik, A.A., Lourdel, S., and Jentsch, T.J. (2005). Voltage-dependent electrogenic chloride/proton exchange by endosomal CLC proteins. Nature 436, 424-427.

Schiavo, G., Matteoli, M., and Montecucco, C. (2000). Neurotoxins affecting neuroexocytosis. Physiol Rev 80, 717-766.

Schneider, D.L. (1987). The proton pump ATPase of lysosomes and related organelles of the vacuolar apparatus. Biochim Biophys Acta 895, 1-10.

Seal, R.P., Akil, O., Yi, E., Weber, C.M., Grant, L., Yoo, J., Clause, A., Kandler, K., Noebels, J.L., Glowatzki, E., et al. (2008). Sensorineural deafness and seizures in mice lacking vesicular glutamate transporter 3. Neuron 57, 263-275.

Shioi, J., and Ueda, T. (1990). Artificially imposed electrical potentials drive L-glutamate uptake into synaptic vesicles of bovine cerebral cortex. Biochem J 267, 63-68.

Slepnev, V.I., and De Camilli, P. (2000). Accessory factors in clathrin-dependent synaptic vesicle endocytosis. Nat Rev Neurosci 1, 161-172.

Smith, S.M., Renden, R., and von Gersdorff, H. (2008). Synaptic vesicle endocytosis: fast and slow modes of membrane retrieval. Trends Neurosci 31, 559-568.

Song, H., Ming, G., Fon, E., Bellocchio, E., Edwards, R.H., and Poo, M. (1997). Expression of a putative vesicular acetylcholine transporter facilitates quantal transmitter packaging. Neuron 18 , $815-826$

Sonnhammer, E.L., von Heijne, G., and Krogh, A. (1998). A hidden Markov model for predicting transmembrane helices in protein sequences. Proc Int Conf Intell Syst Mol Biol 6, 175-182. 


\section{REFERENCES}

Stobrawa, S.M., Breiderhoff, T., Takamori, S., Engel, D., Schweizer, M., Zdebik, A.A., Bosl, M.R.,

Ruether, K., Jahn, H., Draguhn, A., et al. (2001). Disruption of ClC-3, a chloride channel expressed on synaptic vesicles, leads to a loss of the hippocampus. Neuron 29, 185-196.

Sudhof, T.C. (2004). The synaptic vesicle cycle. Annu Rev Neurosci 27, 509-547.

Sudhof, T.C., Cowan, W.M., and Stevens, C.F.: Synapses, Johns Hopkins, 2003

Sudhof, T.C., Lottspeich, F., Greengard, P., Mehl, E., and Jahn, R. (1987). A synaptic vesicle protein with a novel cytoplasmic domain and four transmembrane regions. Science 238, 1142-1144.

Sudhof, T.C., and Rothman, J.E. (2009). Membrane fusion: grappling with SNARE and SM proteins. Science $323,474-477$.

Sutton, R.B., Fasshauer, D., Jahn, R., and Brunger, A.T. (1998). Crystal structure of a SNARE complex involved in synaptic exocytosis at 2.4 A resolution. Nature 395, 347-353.

Suzuki, T., Ueno, H., Mitome, N., Suzuki, J., and Yoshida, M. (2002). F(0) of ATP synthase is a rotary proton channel. Obligatory coupling of proton translocation with rotation of c-subunit ring. $\mathrm{J}$ Biol Chem 277, 13281-13285.

Tabb, J.S., Kish, P.E., Van Dyke, R., and Ueda, T. (1992). Glutamate transport into synaptic vesicles. Roles of membrane potential, $\mathrm{pH}$ gradient, and intravesicular $\mathrm{pH}$. J Biol Chem 267, 15412-15418.

Takamori, S. (2006). VGLUTs: 'exciting' times for glutamatergic research? Neurosci Res 55, 343351.

Takamori, S., Holt, M., Stenius, K., Lemke, E.A., Gronborg, M., Riedel, D., Urlaub, H., Schenck, S., Brugger, B., Ringler, P., et al. (2006). Molecular anatomy of a trafficking organelle. Cell 127, 831-846.

Takamori, S., Malherbe, P., Broger, C., and Jahn, R. (2002). Molecular cloning and functional characterization of human vesicular glutamate transporter 3. EMBO Rep 3, 798-803. 


\section{REFERENCES}

Takamori, S., Rhee, J.S., Rosenmund, C., and Jahn, R. (2000a). Identification of a vesicular glutamate transporter that defines a glutamatergic phenotype in neurons. Nature 407, 189-194.

Takamori, S., Rhee, J.S., Rosenmund, C., and Jahn, R. (2001). Identification of differentiationassociated brain-specific phosphate transporter as a second vesicular glutamate transporter (VGLUT2). J Neurosci 21, RC182.

Takamori, S., Riedel, D., and Jahn, R. (2000b). Immunoisolation of GABA-specific synaptic vesicles defines a functionally distinct subset of synaptic vesicles. J Neurosci 20, 4904-4911.

Tartakoff, A., Vassalli, P., and Detraz, M. (1978). Comparative studies of intracellular transport of secretory proteins. J Cell Biol 79, 694-707.

Thomas-Reetz, A., Hell, J.W., During, M.J., Walch-Solimena, C., Jahn, R., and De Camilli, P. (1993). A gamma-aminobutyric acid transporter driven by a proton pump is present in synaptic-like microvesicles of pancreatic beta cells. Proc Natl Acad Sci U S A 90, 5317-5321.

Thorn, P., and Martin, R.J. (1987). A high-conductance calcium-dependent chloride channel in Ascaris suum muscle. Q J Exp Physiol 72, 31-49.

Tordera, R.M., Totterdell, S., Wojcik, S.M., Brose, N., Elizalde, N., Lasheras, B., and Del Rio, J. (2007). Enhanced anxiety, depressive-like behaviour and impaired recognition memory in mice with reduced expression of the vesicular glutamate transporter 1 (VGLUT1). Eur J Neurosci 25, 281-290.

Towbin, H., Staehelin, T., and Gordon, J. (1979). Electrophoretic transfer of proteins from polyacrylamide gels to nitrocellulose sheets: procedure and some applications. Proc Natl Acad Sci U S A 76, 4350-4354.

Varoqui, H., Schafer, M.K., Zhu, H., Weihe, E., and Erickson, J.D. (2002). Identification of the differentiation-associated $\mathrm{Na}+/ \mathrm{PI}$ transporter as a novel vesicular glutamate transporter expressed in a distinct set of glutamatergic synapses. J Neurosci 22, 142-155. 


\section{REFERENCES}

Voglmaier, S.M., Kam, K., Yang, H., Fortin, D.L., Hua, Z., Nicoll, R.A., and Edwards, R.H. (2006). Distinct endocytic pathways control the rate and extent of synaptic vesicle protein recycling. Neuron 51, 71-84.

Whittaker, V.P., and Sheridan, M.N. (1965). The Morphology and Acetylcholine Content of Isolated Cerebral Cortical Synaptic Vesicles. J Neurochem 12, 363-372.

Wightman, R.M., Schroeder, T.J., Finnegan, J.M., Ciolkowski, E.L., and Pihel, K. (1995). Time course of release of catecholamines from individual vesicles during exocytosis at adrenal medullary cells. Biophys J 68, 383-390.

Wilson, D.S., Keefe, A.D., and Szostak, J.W. (2001). The use of mRNA display to select highaffinity protein-binding peptides. Proc Natl Acad Sci U S A 98, 3750-3755.

Wilson, N.R., Kang, J., Hueske, E.V., Leung, T., Varoqui, H., Murnick, J.G., Erickson, J.D., and Liu, G. (2005). Presynaptic regulation of quantal size by the vesicular glutamate transporter VGLUT1. J Neurosci 25, 6221-6234.

Winter, S., Brunk, I., Walther, D.J., Holtje, M., Jiang, M., Peter, J.U., Takamori, S., Jahn, R., Birnbaumer, L., and Ahnert-Hilger, G. (2005). Galphao2 regulates vesicular glutamate transporter activity by changing its chloride dependence. J Neurosci 25, 4672-4680.

Wojcik, S.M., Katsurabayashi, S., Guillemin, I., Friauf, E., Rosenmund, C., Brose, N., and Rhee, J.S. (2006). A shared vesicular carrier allows synaptic corelease of GABA and glycine. Neuron 50, 575-587.

Wojcik, S.M., Rhee, J.S., Herzog, E., Sigler, A., Jahn, R., Takamori, S., Brose, N., and Rosenmund, C. (2004). An essential role for vesicular glutamate transporter 1 (VGLUT1) in postnatal development and control of quantal size. Proc Natl Acad Sci U S A 101, 7158-7163.

Wolosker, H., de Souza, D.O., and de Meis, L. (1996). Regulation of glutamate transport into synaptic vesicles by chloride and proton gradient. J Biol Chem 271, 11726-11731.

Xie, X.S., Crider, B.P., and Stone, D.K. (1989). Isolation and reconstitution of the chloride transporter of clathrin-coated vesicles. J Biol Chem 264, 18870-18873. 


\section{REFERENCES}

Xie, X.S., and Stone, D.K. (1986). Isolation and reconstitution of the clathrin-coated vesicle proton translocating complex. J Biol Chem 261, 2492-2495.

Xie, X.S., Stone, D.K., and Racker, E. (1983). Determinants of clathrin-coated vesicle acidification. J Biol Chem 258, 14834-14838.

Xie, X.S., Tsai, S.J., and Stone, D.K. (1986). Lipid requirements for reconstitution of the protontranslocating complex of clathrin-coated vesicles. Proc Natl Acad Sci U S A 83, 8913-8917.

Yoshida, S., Ina, A., Konno, J., Wu, T., Shutoh, F., Nogami, H., and Hisano, S. (2008). The ontogenic expressions of multiple vesicular glutamate transporters during postnatal development of rat pineal gland. Neuroscience 152, 407-416.

Yoshikawa, M., Uchida, S., Ezaki, J., Rai, T., Hayama, A., Kobayashi, K., Kida, Y., Noda, M., Koike, M., Uchiyama, Y., et al. (2002). CLC-3 deficiency leads to phenotypes similar to human neuronal ceroid lipofuscinosis. Genes Cells 7, 597-605.

Zerial, M., and McBride, H. (2001). Rab proteins as membrane organizers. Nat Rev Mol Cell Biol $2,107-117$. 


\section{List of Publications:}

Takamori, S., Holt, M., Stenius, K., Lemke, E.A., Gronborg, M., Riedel, D., Urlaub, H., Schenck, S., Brugger, B., Ringler, P., Müller, S.A., Rammner, B., Gräter, F., Hub, J.S., De Groot, B.L., Mieskes, G., Moriyama, Y., Klingauf, J., Grubmüller, H., Heuser, J., Wieland, F., and Jahn, R. . (2006). Molecular anatomy of a trafficking organelle. Cell 127, 831846.

Schenck, S., Wojcik, S.M., Brose, N., and Takamori, S. . (2009) A chloride conductance in VGLUT1 underlies maximal glutamate loading into synaptic vesicles. Nat Neurosci $12,156-162$ 


\section{ACKNOWLEDGEMENTS}

First, I wish to thank Dr. Shigeo Takamori for introducing me to this interesting and challenging project. Without him this work would not have been possible. I appreciate his motivating will to tackle also difficult problems.

I am thankful for your understanding and support during my stay in Japan, also outside the lab. Because of you I could expirience life in Japan something to remember for a lifetime.

Thank you Shigeo!

I thank Prof. Reinhard Jahn for the first two years of my doctoral studies in his laboratory, an outstanding place to do science. I further greatly appreciate his support for my decision to continue my work in Japan, providing important tools and good scientific advices. Furthermore I appreciate his willingness to help in difficult times.

My thanks go to Dr. Mitsuko Hirosawa-Takamori who greatly helped me with my stay in Japan and took over many responsibilities with great care. I further thank you, Mitsuko, for introducing me to your family and showing me japanese life. I enjoyed the pleasant stays in Yokosuka with very delicious food and the happy atmosphere.

I thank all the members of the Jahn-lab during my time in Göttingen for it was my start in science that I expirienced with them.

Mein besonders herzlicher Dank natürlich an Alexander. Es war eine große Freude mit Dir im Labor zu sein! Und auch mit Dir aus dem Labor rauszugehen.

Ich möchte auch Gottfried Mieskes für seine verständnisvolle und hilfsbereite Art danken.

I thank David and Quy for the good times we had in Tokyo !

My thanks go to Dr. Hiroshi Kawabe for the translation of the abstract of this work into Japanese language!

Ich bin sehr dankbar für das Verständnis meiner Freunde in Hamburg, die mich nicht vergessen haben obwohl ich so lange fort war: Antje, Hein Mück und Axel.

Mein ganz besonderer Dank gilt meiner Mutter Aspasia und meinem Bruder Daniel:

Ich danke Euch sehr für die Unterstützung die ihr mir gegeben habt. Wo hätte ich sonst neuen Mut bekommen können wenn nicht durch Eure lieben Worte und Euer Vertrauen. Vielen Dank!

Göttingen, Mai 2009 


\section{Lebenslauf}

Name:

Geburtstag:

Geburtsort:

1994:

1994-1995:

1996-2001:

2001-2002:

2002-2004:

2004-2009:
Stephan Schenck

30.04 .1975

Hamburg

Erreichen der Hochschulreife am Gymnasium Finkenwerder, Hamburg

Zivildienst als Krankenpfleger am Allgemeinen Krankenhaus Altona, Hamburg

Studium der Biologie an der Universität Hamburg

Diplom an der Universität Hamburg bei Prof. Hagen Bretting (Hauptfach Zoologie), externe Arbeit am Hans-Knöll-Institut für Naturstoffforschung in Jena in Zusammenarbeit mit Prof. Peter F. Zipfel

Beginn der Promotion im Max-Planck-Institut für biophysikalische Chemie, Göttingen. (Abteilung Neurobiologie, Prof. Reinhard Jahn)

Fortsetzung der Promotion an der Tokyo Medical and Dental University in Tokyo, Department of Neurology, bei Prof. Shigeo Takamori 